\title{
Nanocatalysis: Size- and Shape-dependent Chemisorption and Catalytic Reactivity
}

\author{
Beatriz Roldan Cuenya, ${ }^{1 *}$ Farzad Behafarid ${ }^{2}$
}

\author{
${ }^{1}$ Department of Physics, Ruhr University Bochum, Bochum 44801, Germany \\ ${ }^{2}$ Department of Physics, University of Central Florida, Orlando, FL 32816, USA \\ *e-mail: Beatriz.Roldan@rub.de
}

\begin{abstract}
:
In recent years, the field of catalysis has experienced an astonishing transformation, driven in part by more demanding environmental standards and critical societal and industrial needs such as the search for alternative energy sources. Thanks to the advent of nanotechnology, major steps have been made towards the rational design of novel catalysts. Striking new catalytic properties, including greatly enhanced reactivities and selectivities, have been reported for nanoparticle (NP) catalysts as compared to their bulk counterparts. However, in order to harness the power of these nanocatalysts, a detailed understanding of the origin of their enhanced performance is needed. The present review focuses on the role of the NP size and shape on chemisorption and catalytic performance. Since homogeneity in NP size and shape is a prerequisite for the understanding of structure-reactivity correlations, we first review different synthesis methods that result in narrow NP size distributions and shape controlled NPs. Next, size-dependent phenomena which influence the chemical reactivity of NPs, including quantum size-effects and the presence of undercoordinated surface atoms are examined. The effect of the NP shape on catalytic performance is discussed and explained based on the existence of different atomic structures on the NP surface with distinct chemisorption properties. The influence of additional factors such as the oxidation state of the NPs and NP-support interactions, is also considered in the frame of the size- and shape-dependency that these phenomena present. Ultimately, our review highlights the importance of achieving a systematic understanding of the factors that control the activity and selectivity of a catalyst in order to avoid trial and error methods in the rational design of the new generation of nanocatalysts with properties tunable at the atomic level.
\end{abstract}




\section{Content}

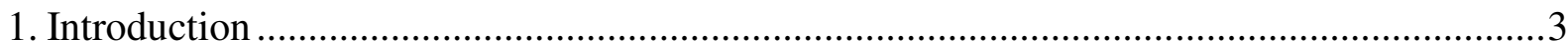

2. Synthesis methods resulting in size- and shape-selected nanostructures..........................4

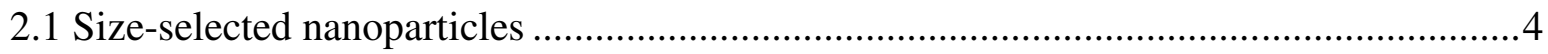

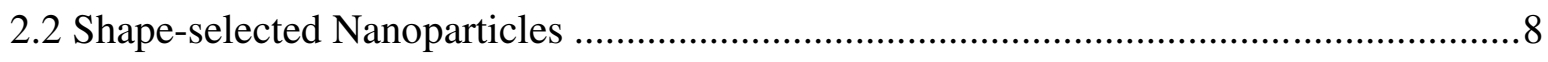

a) Kinetically metastable shapes ....................................................................... 8

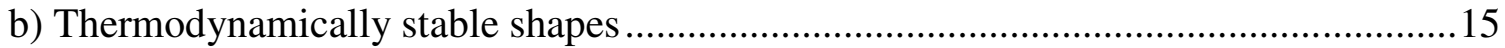

c) Evolution of the NP structure under reaction conditions........................................ 18

3. Role of the nanoparticle size on catalytic activity and selectivity ..................................26

3.1 Quantum size-effects.......................................................................................... 50

3.2 Undercoordinated atoms at the NP surface .......................................................57

3.3 NP-support interaction and charge transfer ...........................................................63

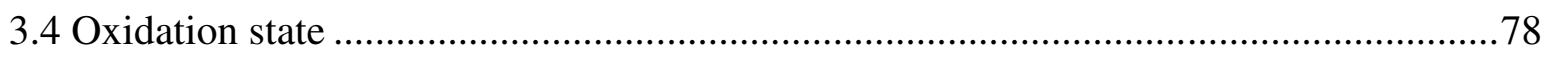

4. Role of the nanoparticle shape on catalytic activity and selectivity ...............................90

4.1. Effect of different surface facets ....................................................................... 90

4.2. Other shape-related effects uniquely observed for NPs ....................................... 94

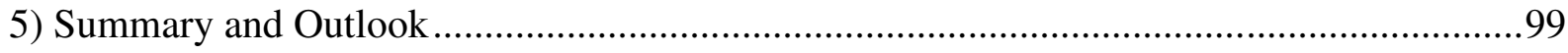

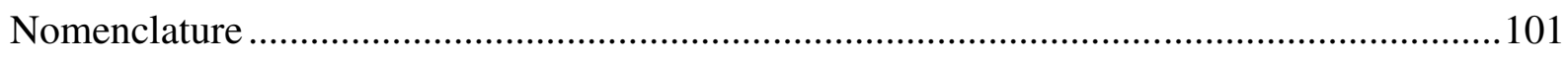

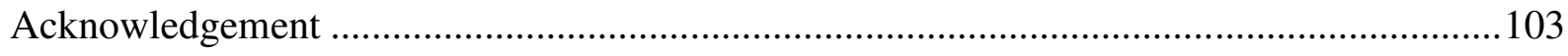

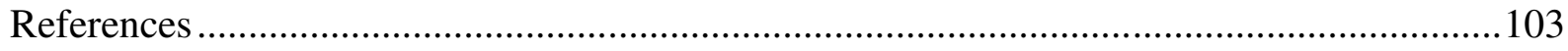




\section{Introduction}

Unraveling the complex interaction between catalysts and reactants and tailoring their chemical reactivity at the atomic level are key steps towards gaining fundamental insight in catalysis. Such knowledge could enable the rational design of the next generation of highly active, selective, and stable nanosized catalysts for industrial applications. Consequently, intense effort has been exerted towards understanding the properties that affect the catalytic performance of metal nanoparticles (NPs) such as their size and shape [1-9], interaction with the support [10-13], and oxidation state [14-16]. In order to deconvolute the influence of each of these NP properties on catalyst reactivity, model systems with highly tunable structure must be synthesized and tested under controlled environments.

Arguably, the most important property to understand is the influence of NP morphology on catalytic ability. NP size and in particular shape will determine the availability of certain active facets or low coordinated sites on the NP surface, which can affect the binding strength of reactants and intermediates and thereby the activity and selectivity of a particular reaction. In addition, NP morphology will affect the degree of interaction of the metal with the support, influencing charge transfer phenomena or the extent of interfacial strain. While size control has been readily achieved for nanocatalysts, shape control, particularly for NPs below $10 \mathrm{~nm}$, has been much more challenging. However, new synthesis methods have allowed progress in this area [9].

Further complexity is added to understanding the origin of NP catalytic properties when catalysts are considered under realistic reaction environments. NPs which are carefully synthesized with well-defined size and shape may evolve in morphology during a reaction in response to elevated temperatures or the chemisorption of gas molecules [17-19], meaning that structural and chemical characterization which is performed before and after a reaction may not reflect the actual state of the catalyst during the reaction. Consequently, in-situ and operando measurement techniques that can bridge this pressure gap have become increasingly important to understanding the dynamic nature of active NP catalysts and their response to their environment $[20 ; 21]$.

This review article focuses on correlating the catalytic reactivity of NPs and their geometry. It will be illustrated that chemisorption and catalytic properties such as the onset reaction temperature, the activity, and selectivity of a nanocatalyst may be tuned through controlled synthesis of NPs with well-defined sizes and shapes. 


\section{Synthesis methods resulting in size- and shape-selected nanostructures}

In order to understand and further harvest the potential novel chemical properties of nanostructured materials in the field of catalysis, significant effort needs to be made in the years to come towards the synthesis of structurally well-defined systems, including size- and shapeselected nanoparticles. A variety of approaches have been used in the past for the synthesis of nanoparticles, such as thermal evaporation in vacuum [22-27], atomic layer deposition [28-31], electron-beam lithography [32-35], nanosphere lithography [35-39], pulsed laser deposition [34; 40; 41], buffer-layer assisted growth [42-44], chemical vapor deposition [45-47], gas condensation, ionized cluster beam deposition [48-52], electrochemical deposition [53-57] ,sol-gel or colloidal techniques [58-60], deposition-precipitation and impregnation methods [6;61;62], molecular cluster precursors [63], or solvothermal synthesis [64-66].

Nevertheless, only a few of the above methods are suitable for the growth of nanostructures with controlled size and shape and uniform dispersion over the support surface. Moreover, reproducible and low cost synthetic approaches leading to robust nanostructures which can preserve their pre-defined morphological features under reaction conditions (i.e. upon exposure to reactants, at elevated temperatures, and under pressure) must be sought. An additional challenge is developing methods which are scalable to large substrate areas and material quantities in order to be transferred to an industrial setting. Encouraging progress along these lines has been made through the utilization of organic ligands for the confinement of inorganic catalysts in dendrimeric [67-69] and micellar nanoreactors [70-73] with predefined sizes and shapes, or through the use of porous matrixes as encapsulating media [74-81]. The sections below describe recent advances in the synthesis of size- and shape-controlled nanostructures.

\subsection{Size-selected nanoparticles}

An established technique for the synthesis of size-selected NPs is based on the use of massselective cluster sources, where the clusters are first formed by sputtering and condensation in the gas phase and subsequently mass-filtered before deposition on the substrate, Figure 1(a) [49; 50; 52; 82-85]. Images of NPs prepared using this method are given in Figure 1(b-g). 

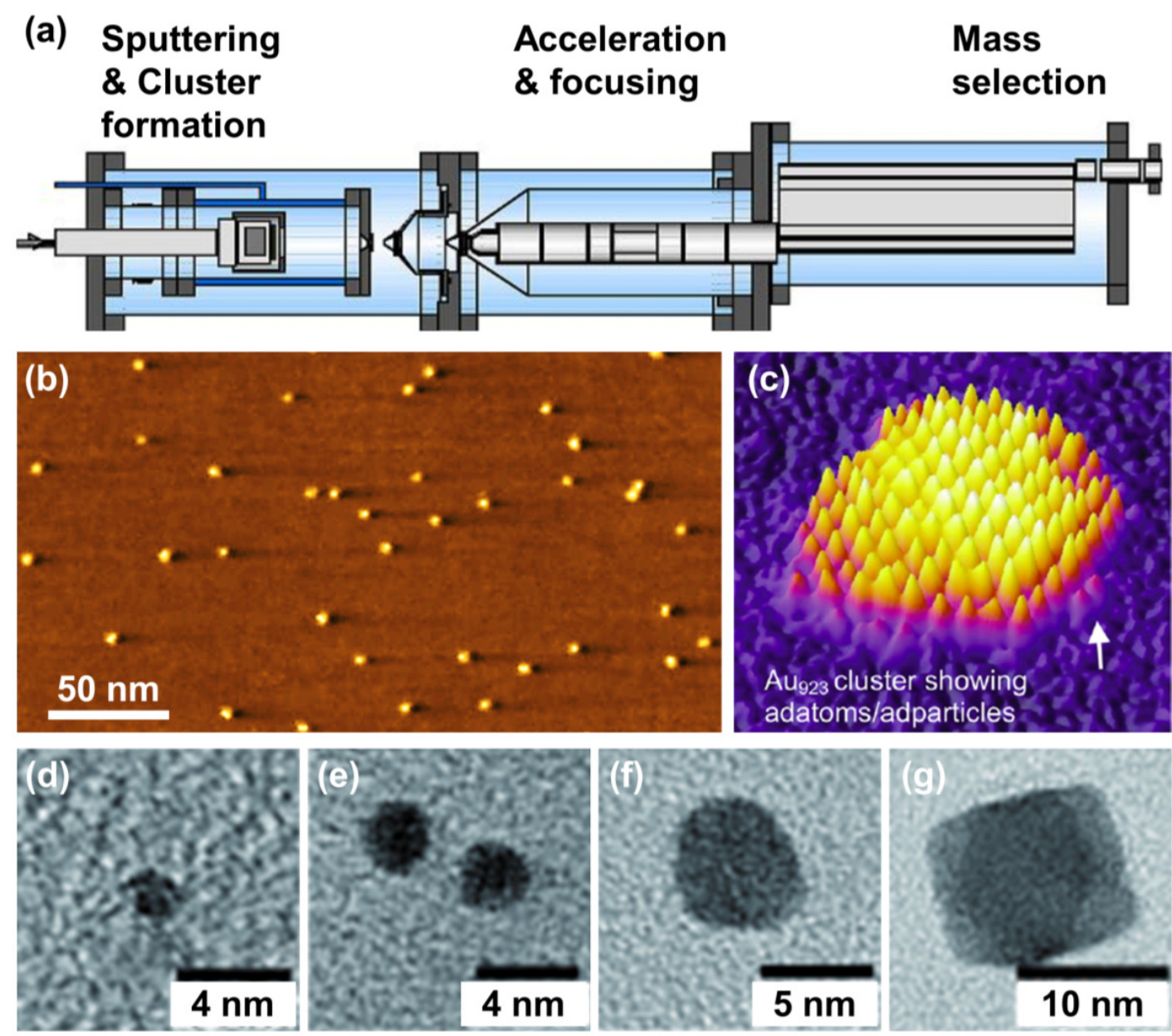

Figure 1: (a) Magnetron sputtering/gas condensation nanocluster beam source [83]. (b) STM image of $\mathrm{Au}_{70}$ nanoclusters supported on graphite [84], and (c) HAADF-STEM image of a truncated octahedral $\mathrm{Au}_{293}$ nanocluster synthesized using this method [52]. (d-g) High resolution (HR) TEM images of mass-selected Pt NPs supported on glassy carbon synthesized via the gasphase aggregation technique [86]. Adapted from Refs. [52; 83; 84; 86].

Colloidal chemistry has been proven to be a powerful and versatile technique for NP synthesis [14; 65; 87-110]. Figure 2 shows examples of size-selected Co and Fe oxide NPs prepared using colloidal chemistry and imaged by TEM. The iron oxide species in the NPs shown in Figure 2(d-e) are a combination of $\gamma-\mathrm{Fe}_{2} \mathrm{O}_{3}$ and $\mathrm{Fe}_{3} \mathrm{O}_{4}$, depending on the NP size [108]. 

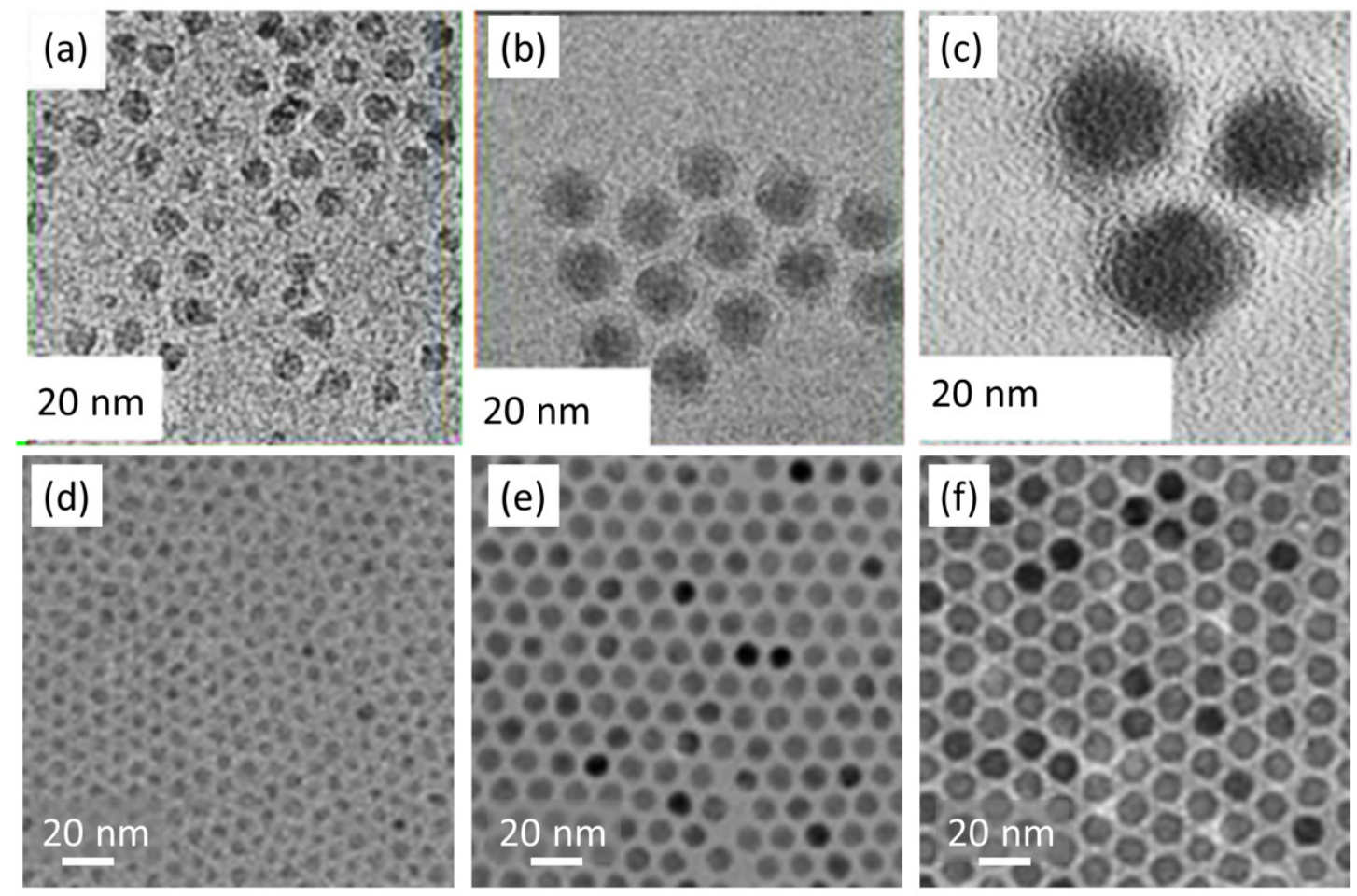

Figure 2: Size-selected NPs: (a-c) HR-TEM images of Co NPs prepared using trioctylphosphine oxide (TOPO). The particle size was varied here by changing the temperature of the solution into which $\mathrm{Co}_{2}(\mathrm{CO})_{8}$ was injected. The average NP sizes from left to right are $4.8 \pm 0.3 \mathrm{~nm}, 6.8 \pm 0.5$ $\mathrm{nm}$, and $10.2 \pm 0.6 \mathrm{~nm}$ [107]. (d-f) TEM images of air-oxidized iron oxide NPs: (d) $6 \mathrm{~nm}$, (e) 9 $\mathrm{nm}$, and (f) $13 \mathrm{~nm}$. In the first step of the synthesis, monodisperse iron NPs with sizes of 4, 8, and $11 \mathrm{~nm}$ were synthesized from reaction mixtures containing different molar ratios of pentacarbonyliron and oleic acid. After refluxing the mixtures generated from the various combinations of the iron NPs and the iron oleate solutions, monodisperse iron NPs with sizes ranging from 6-15 nm were obtained [108]. Adapted from Refs. [107; 108].

Figure 3 shows a colloidal synthesis technique employing PS-P2VP diblock-copolymers to generate reverse micelles which are subsequently loaded with a metal precursor $[3 ; 14 ; 70 ; 100$; 111-115]. This method gives rise to size-selected NPs whose size is controlled by the length of the polymer head (P2VP group) and by the metal precursor to P2VP molecular weight ratio (L). Furthermore, the initially spherical micelles generated can lead to either 3D-like NPs or flatter 2Dlike nanostructures by tuning the following parameters: (i) the metal/P2VP ratio (L), with flatter NPs achieved for low metal loadings inside the micellar cages, and (ii) the type of support employed, with strongly interacting substrates favoring the stabilization of 2D-like NPs upon certain annealing pre-treatments, and (iii) the possible NP/support interfacial epitaxial match. 

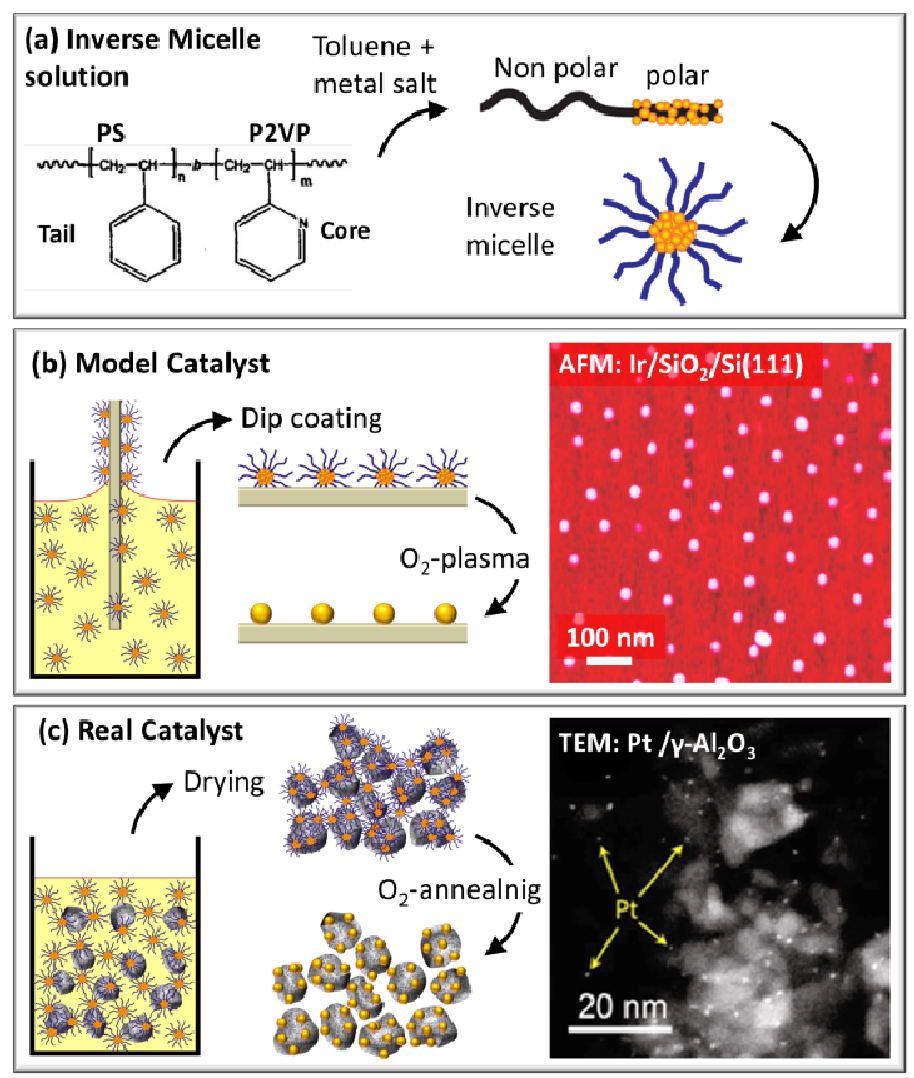

Figure 3: (a) Schematic of the inverse micelle encapsulation NP synthesis method. A NP solution containing reverse micelles is prepared by dissolving a PS-P2VP diblock copolymer in toluene and subsequently adding a metal salt. The micellar cages that are loaded with metal atoms in the core are separated from each other by the polymeric ligands. (b) Deposition of one monolayer of micelles self-assembled in a hexagonal pattern using a dip-coating method. The polymer is then removed using an $\mathrm{O}_{2}$-plasma treatment. An AFM image of Ir NPs supported on $\mathrm{SiO}_{2} / \mathrm{Si}(111)$ is shown on the right [14]. (c) Deposition of micellar NPs on high surface area nanocrystalline powder supports by impregnation and air drying. The removal of the polymeric ligands is achieved by annealing in $\mathrm{O}_{2}\left(24 \mathrm{~h}, 375^{\circ} \mathrm{C}\right)$. A HAADF-TEM example of size-selected Pt NPs $(\sim 0.8 \mathrm{~nm})$ supported on $\gamma-\mathrm{Al}_{2} \mathrm{O}_{3}$ is provided on the right [3]. Adapted from Refs. [3; 14].

Another approach to synthesize NPs with narrow size distribution or to deposit the pre-made NPs on a substrate in a spatially uniform fashion is to use pre-patterned surfaces [116-126]. In these cases, local defects and the variation in surface potentials favor the attachment of metal atoms or NPs to specific sites (e.g. potential wells) on the surface. NPs could form either on a surface that intrinsically has a periodic structure or on surfaces that are patterned using a variety of different methods. For instance, by taking advantage of the surface structure of $\operatorname{Si}(111)-(7 \times 7)$, highly uniform nanocluster arrays of In and Mn could be formed using physical vapor deposition [122]. The $7 \times 7$ reconstruction of the $\mathrm{Si}(111)$ surface provides a large lattice unit that is appropriate for nanocluster formation. Another approach is to use the Moiré pattern formed on surfaces that 
generally have large lattice units. Such patterns appear on the surface due to the lattice mismatch of the upper thin film and the support underneath. In particular, the Moire patterns formed on boron nitride thin films supported on $\mathrm{Ru}(0001)$ [127] and $\mathrm{Rh}(111)$ [128], on graphene layers deposited on $\mathrm{Ru}(0001)$ [124] and $\operatorname{Ir}(111)$ [129] and on FeO layer deposited on $\mathrm{Pt}(111)$ [130] could be used to fabricate small well defined NPs.

In addition, localized NP nucleation sites on pre-patterned surfaces could also result in highly dispersed NPs [131-135]. For instance ion bombardment and controlled oxygen etching were used to form nanopits on graphene surfaces in order to anchor well-dispersed Au and Pd NPs [133-135].

Another extensively used method to pre-pattern surfaces is lithography, that includes a variety of techniques such as electron beam lithography [32-35], nanosphere lithography [36-39], and nano-imprinting [136-138]. In addition, there have been approaches to synthesize NPs that are not limited by the pattern resolution and could be much smaller than the pattern lattice [139]. For instance, a surface that is patterned to hydrophilic and hydrophobic domains could selectively collect a metal- salt containing aqueous liquid on hydrophobic regions. The evaporation of the solvent leaves behind metal NPs with uniform size [139]. If the sample is held vertically, the accumulation of metal salt during the droplet evaporation would occur on the lower portion of the hydrophilic area, resulting in NPs that are much smaller than the size of the hydrophilic domain [139]. Although the lithographic approach is an excellent route to "top-down" fabrication of large

surface area samples with uniform NP size and well defined spatial distribution, it is often costly and not well suited to make NPs smaller than $20 \mathrm{~nm}$. To overcome this limitation and achieve smaller NP sizes, biologically derived mask [123], or self-assembled block copolymer lithography [140-142], have been also used to pre-pattern support surfaces.

The initial size-selection of NPs may not be stable under certain environments and at high temperature due to NP growth through Ostwald ripening and/or diffusion-coalescence coarsening pathways [27; 143-145]. Therefore, other factors such as NP size, NP density, pretreatment, and support material should be taken into account to increase the NP stability against coarsening [27; $143 ; 146-149]$.

\subsection{Shape-selected Nanoparticles}

\section{a) Kinetically metastable shapes}


Although the thermodynamically stable shape of NPs of a given size under a given condition is unique, metastable NP shapes could be synthesized kinetically by tuning the nucleation and growth processes.

The size and shape of NPs can be engineered during growth by altering chemical and physical conditions during the synthesis process. The majority of shape-selective colloidal synthesis methods are based on seed-mediated growth. In general, the nucleation process is fast, and requires a super saturated condition with a high reaction rate. However, such conditions are detrimental to the control of the NP shape, since they favor anisotropic growth rates. The growth step is, however, usually a slow process, allowing an isotropic evolution favoring specific facets with the highest reaction rates or facets that are not blocked strongly by capping agents. Such anisotropic growth would determine which facets are featured in the final shape of the synthesized NP. For instance, a capping agent that bonds more strongly to (100) facets would prohibit or slow down the growth of those facets, while NP growth on other facets continues. Following this process, the facets that are growing faster would get smaller in area and eventually disappear, while those that are hindered in growth would become dominant.

A variety of shapes have been reported, including hexahedra (cube), octahedra, truncated octahedra, truncated cubooctahedra, spherical, nanoplates, nanorods, branched nanostructures, concave polyhedra and hollow nanostructures [89; 105; 150-168]. Other shapes such as decahedra or icosahedra (or their derivatives, rods and disks) might also be obtained as a result of twinning in the crystalline structure, minimizing the free surface energy with the cost of increasing the energy in crystalline domain boundaries. For example, decahedral (pentagonal bipyramidal) NPs with five-twin boundaries would feature only $\{111\}$ facets which have the lowest surface energy [150; 169; 170]. Examples of different NP shapes are displayed in Figure 4. 


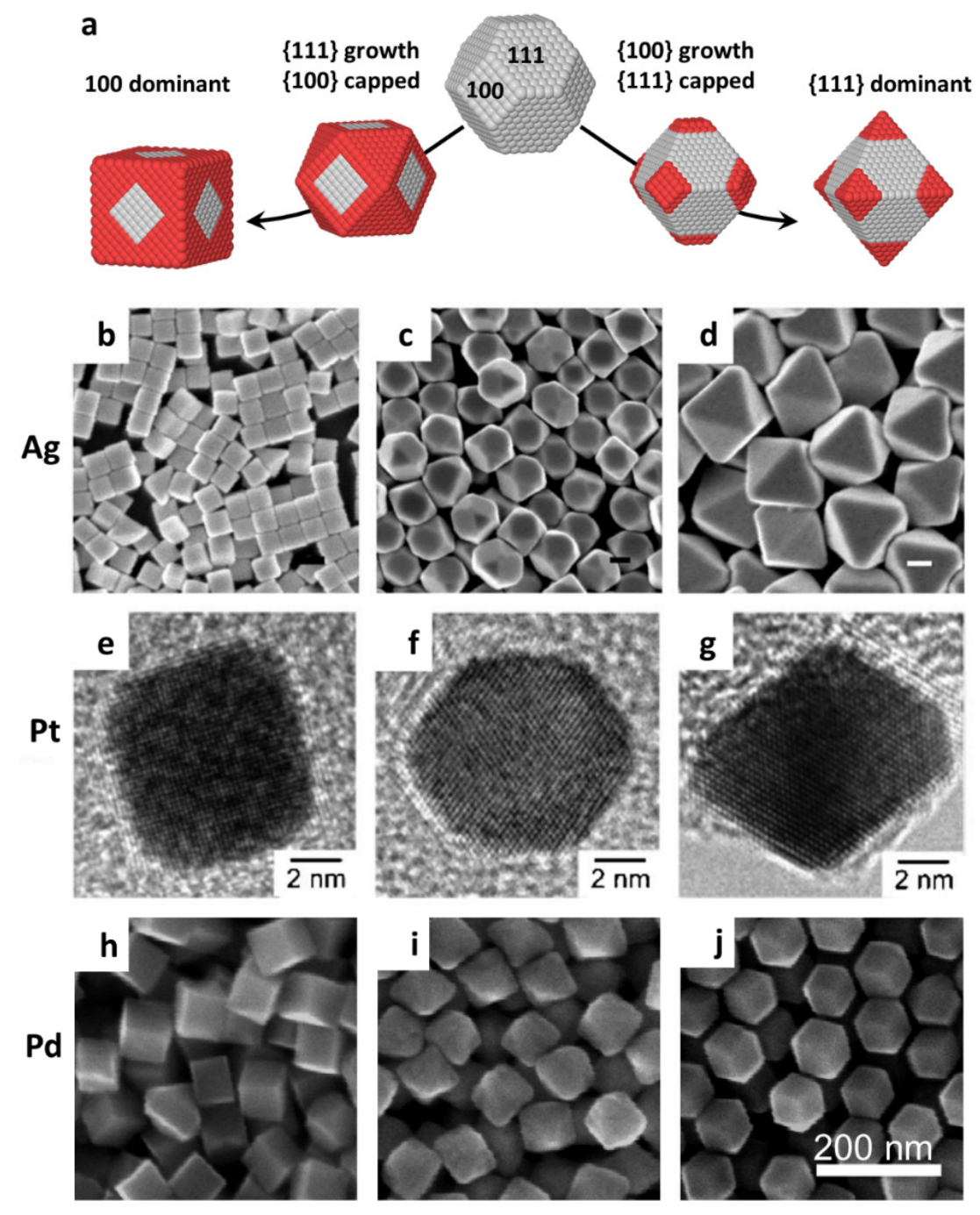

Figure 4 : (a) Model shapes illustrating an anisotropic growth processes starting from a truncated cuboctahedron shape. Protecting the $\{111\}$ facets using a capping agent results in the growth of $\{100\}$ facets until they disappear and only $\{111\}$ facets remain on the surface (octahedron shape). The opposite would result in cubic NPs with only $\{100\}$ facets. Examples of shape-selected Ag (bd) [171] and Pt NPs (e-g) [158] prepared using the polyol method [172] with PVP as the capping agent. The scale bar in (b-d) is $100 \mathrm{~nm}$. (h-j) SEM images of palladium nanocrystals synthesized using a seed-mediated method [160]. In addition to the NPs featuring only $\{100\}$ facets in $(h)$, \{111\} facets in (i) and $\{110\}$ facets in (j), intermediate shapes could also be achieved [160]. Adapted from Refs. [158; 160; 171].

For instance, Sun et al. [173] used poly(vinylpyrrolidone) (PVP) as capping agent to synthesize silver nanocubes $(\sim 100 \mathrm{~nm})$ from the reduction of silver nitrate. The PVP group bonds stronger to 100 facets, leading to cubic NP shapes. However, silver NPs could be used as sacrificial templates to synthesize $\mathrm{Au}$ NPs following an atom-by-atom replacement process in 
which the shape was found to evolve from cubic to cuboctahedron [173]. Ahmadi et. al. [174; 175] showed that $\sim 8 \mathrm{~nm}$ Pt NPs of different shapes could be synthesized by changing the ratio of capping agent to platinum cations, with all the other factors such as the precursor salt, $\mathrm{pH}$, temperature, etc., being kept identical. Small NPs start to form with a tetrahedral shape, but the final shape was found to depend on the competition between the $\mathrm{Pt}^{2+}$ reduction that occurs most efficiently on (111) facets, and the site blockage by the capping agent that also occurs at (111) facets [174]. If the former prevails, the (111) facets grow until they disappear, resulting in the formation of cubic NPs; otherwise, smaller tetrahedral NPs would form [174; 176]. Nevertheless, to achieve shape selection, the nucleation and growth processes should be carefully tuned [177]. For instance, if the rate of the nucleation process of Pt NPs is too fast (e.g. high temperature), the majority of the precursor would be consumed during nucleation, hindering the growth of faceted NPs. In such case, small polyhedron NPs would form [177]. A lower nucleation rate would result in the formation of fewer nuclei, which would allow the generation of well-faceted NPs [177].

Standard synthesis methods, however, do not provide high Miller index facets, since the growth rate of such facets is very fast, resulting in their quick disappearance. Nevertheless, there are approaches such as the electrochemical square-wave potential method to produce high Miller index surfaces [178-181]. Using this method, Tian et al. have synthesized tetrahexahedral Pt NPs with 24 facets of $\operatorname{Pt}(730)$ [178; 181]. Other shapes such as flower-like or concave hexoctahedral Pt NPs, multiple twinned Pt nanorods, and trapezohedral Pd NPs were also synthesized by modifying the growth conditions, see Figure 5 [178; 182]. 


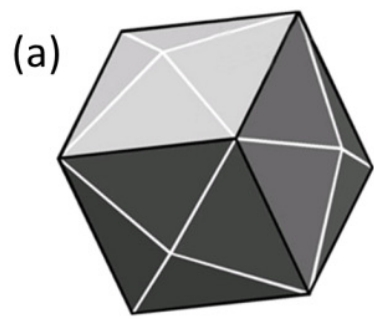

Tetrahexahedron

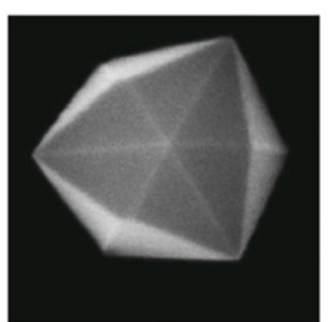

$300 \mathrm{~nm}$
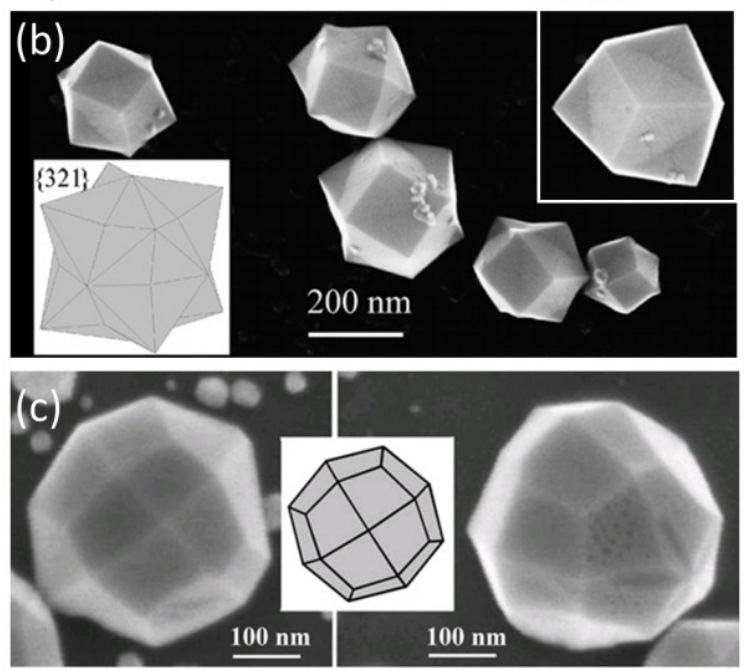

Figure 5: (a) Model of a tetrahexahedron (THH) and atomic arrangement structure of $\{\mathrm{hk} 0\}$ high index planes (left). SEM image of THH Pt nanoclusters (NCs) of different sizes prepared by an electrochemical square-wave potential method and supported on glassy carbon [182]. (b) SEM images of concave hexoctahedral Pt NCs with $\{\mathrm{hkl}\}$ facets. The inset is a model of a concave hexoctahedron shape enclosed by \{321\} facets [182]. (c) SEM images of trapezohedral Pd NCs with $\{\mathrm{hkk}\}$ facets prepared by the electrochemical square-wave potential method and supported on an indium tin oxide (ITO) substrate [182]. Adapted from Ref. [182].

Some of the mentioned synthesis methods have been successfully used to prepare bimetallic nanostructures as well. Figure 6 displays an example of the solvothermal synthesis route used to synthesize shape-selected PtNi octahedral NPs [65; 66; 183; 184]. 


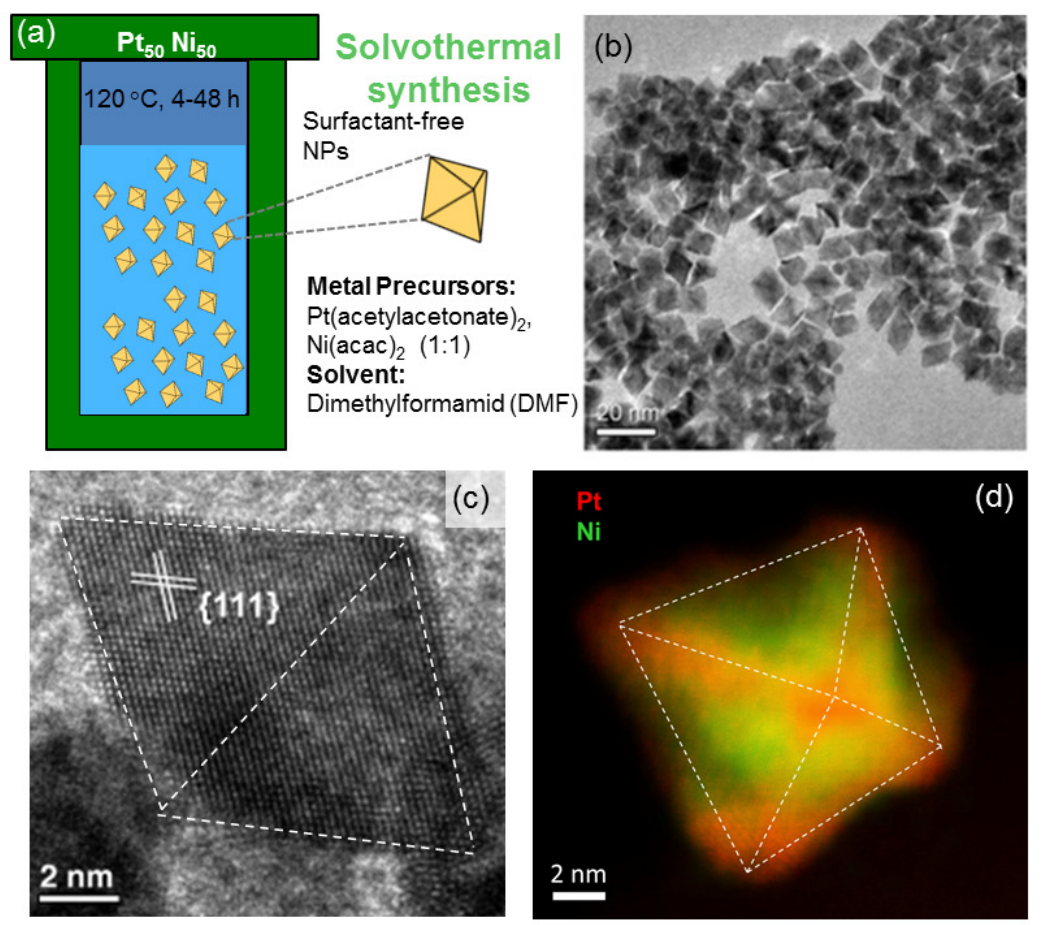

Figure 6 (a) Synthesis of shape-selected PtNi NPs via solvothermal reduction with a single solvent, reducing agent, and surfactant (DMF). (b) TEM image of the as-prepared octahedral $\mathrm{Pt}_{0.5} \mathrm{Ni}_{0.5}$ NPs. (c) High resolution TEM image of a octahedral $\mathrm{Pt}_{0.5} \mathrm{Ni}_{0.5} \mathrm{NP}$ showing $\{111\}$ facets. (d) Electron energy loss spectrum of an octahedral PtNi NP acquired along the [100] zone axis featuring Pt-enrichment (red) at the corner and edges and Ni-enrichment (green) at the faces. Adapted from Refs. [65; 66].

Another method to synthesize complex structures is to start with a bimetallic system and selectively remove one of the elements $[66 ; 162 ; 185-187]$. In Figure 7(a-f), the Ni atoms in octahedral Pt-Ni NPs synthesized by the solvothermal method are leached electrochemically resulting in a concave structure. That structure is shown to change slightly after 4000 potential cycles, but the general shape of the NPs remains. Figure 7(g-i) shows nanocage, nanoframe, and nanotube structures synthesized using selective leaching of one of the components. 


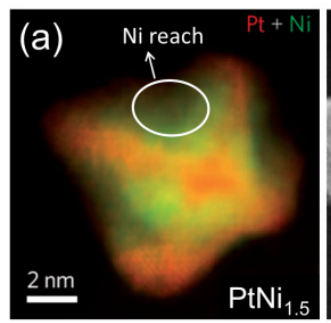

(d) As prepared
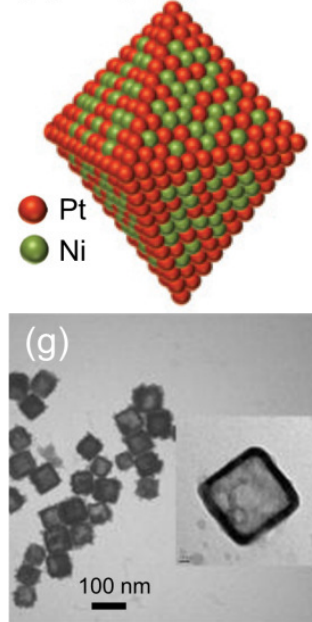

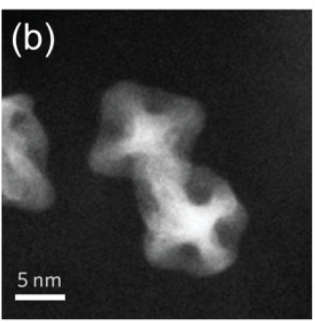

(e) activated
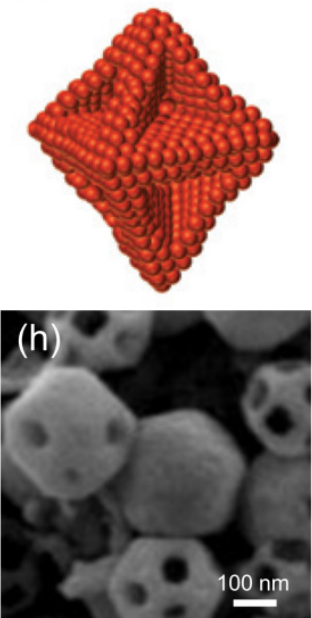

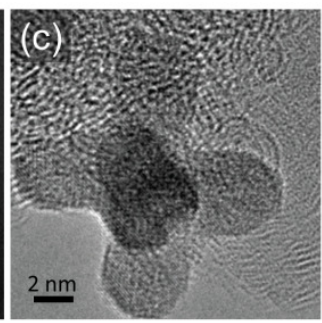

(f) stability test
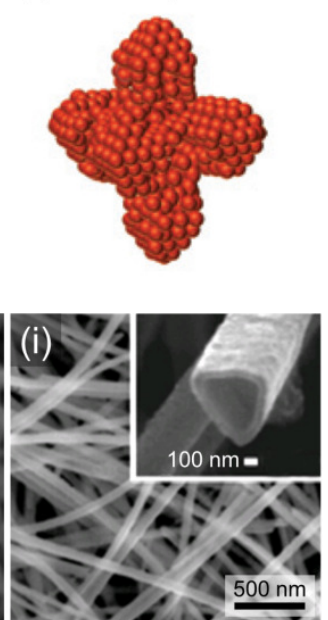

Figure 7: (a,d) TEM image and corresponding model of as-prepared shape-selected octahedral $\mathrm{PtNi}_{1.5}$ NPs synthesized via solvothermal reduction with a single solvent, reducing agent, and surfactant, DMF. The facets are Ni-rich, while the edges/corners are Pt-rich. $(\mathrm{b}, \mathrm{e})$ NPs adopt a concave morphology by selective leaching of $\mathrm{Ni}$, which is achieved electrochemically by 25 potential cycles in the potential regime of $0.06-1.0 \mathrm{~V}_{\mathrm{RHE}}$ at a scan rate of $250 \mathrm{mV} \mathrm{s}^{-1}$. (c,f) NP structure after electrochemical stability tests over the course of 4000 potential cycles $(0.6$ and $1.0 \mathrm{~V}$ at $50 \mathrm{mV} \mathrm{s}^{-1}$ ) [66]. (g) $\mathrm{Pd} / \mathrm{Pt}$ shell-shell nanocages fabricated from silver nanocubes by galvanic replacement of silver atoms by $\mathrm{Pt}$ and $\mathrm{Pd}$ [186]. (h) $\mathrm{Cu}_{2} \mathrm{O}$ nanoframes fabricated by selective etching of $\{110\}$ facets of the initial nanocages [187]. (i) SEM images of Pt nanotubes that were prepared by coating trigonal selenium wires and subsequent dissolution of $\mathrm{Se}$ in hydrazine monohydrate [162]. Adapted from Refs. [66; 162; 186; 187].

Although some of the above methods have shown promising results in producing stable and active nanocatalysts, further work is still needed in order to establish non-aggressive and reproducible approaches for the complete removal of precursor additive traces and undesired encapsulating ligands, to open channels that would help the access of reactants to the active metal sites, or to maximize the available metal NP surface area exposed to the reactants in the case of NPs trapped inside porous supports. Some of these approaches include the use of UV/ozone cleaning, annealing in oxygen, or room-temperature $\mathrm{O}_{2}$-plasma treatments. In addition, it should be ensured that the resulting material system retains the desired geometry. Some examples of the evolution of the NP structure under reaction conditions are given in section (c). 


\section{b) Thermodynamically stable shapes}

Although colloidal chemistry is the most common and tunable method to fabricate size- and shape-selected NPs, the kinetic shapes that are achieved by adjusting the synthesis parameters may not be stable when exposed to thermal treatments or different chemical (gaseous or liquid) environments.

Figure 8 shows an example of how tetrahedral Pt NPs prepared using colloidal chemistry lose their original shape and become rounded upon annealing at $575 \mathrm{~K}$ [188].

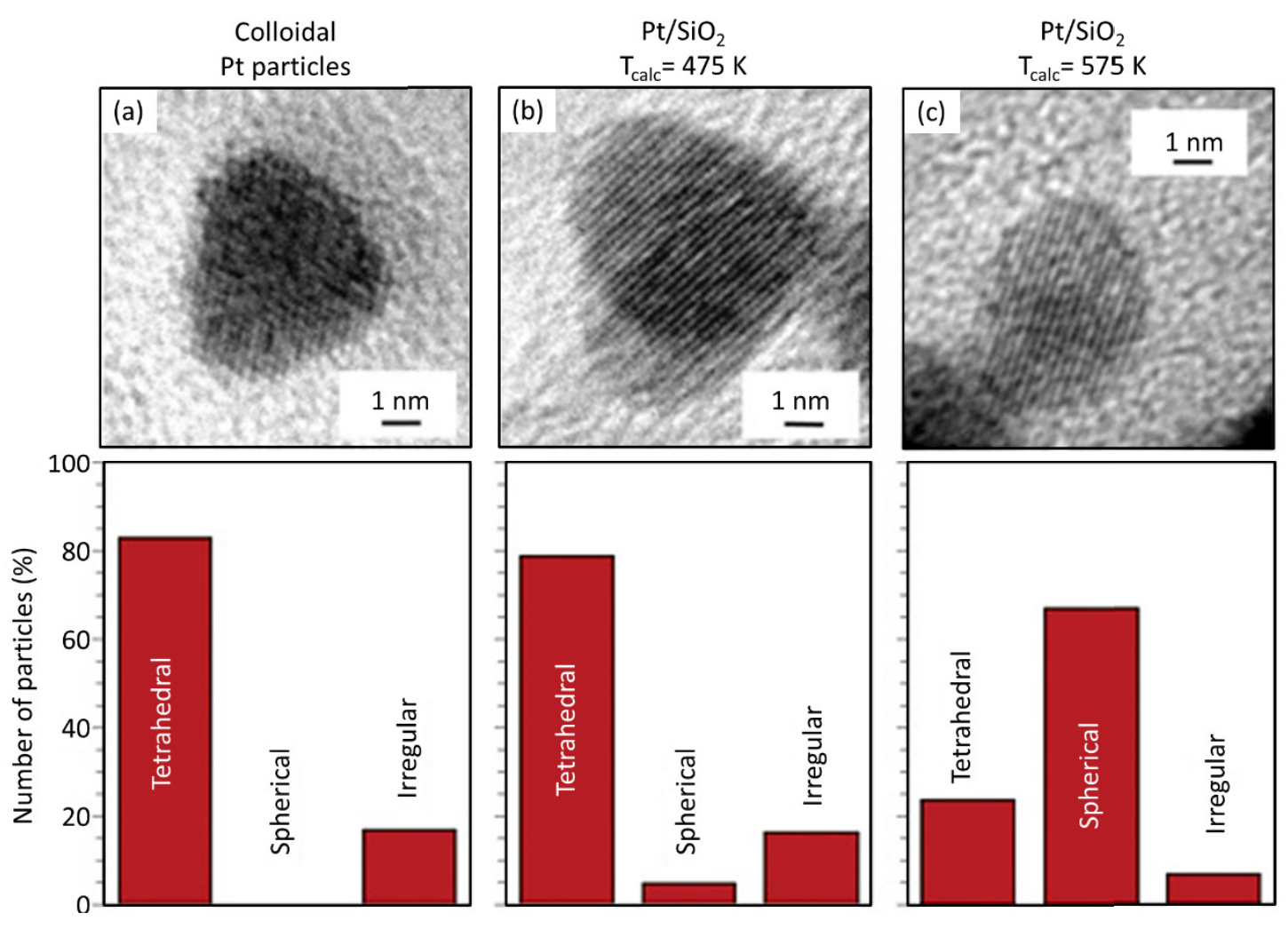

Figure 8: TEM images of Pt NPs immediately after their growth in colloidal solutions (a), and after their dispersion on a silica xerogel support and calcination at $T_{\text {calc }}=475 \mathrm{~K}$ (b), and $575 \mathrm{~K}$ (c). The high-resolution TEM images highlight the change in the supported Pt NPs towards a more round shape and the loss of (111) facets after high temperature treatments. The shape transition occurs without any significant variation in particle size. The number of NPs with a given shape after the different treatments is shown below each TEM image. Adapted from Ref. [188]

The most thermodynamically favored shapes of large enough single crystal NPs can be obtained based on the Wulff theorem [189], in which different crystallographic facets appear at a distance from the center of the particle $\left(d_{i}\right)$ that is proportional to their surface energy $\left(\gamma_{i}\right)$. Therefore, the higher the surface energy, the further away that particular facet should appear $\left(\mathrm{d}_{\mathrm{i}} / \gamma_{\mathrm{i}}\right.$ $=$ constant $)$. The shape of the particle is then determined by the facets that are closest to the NP 
center. The Wulff structure depends on both the type of metal and its crystalline structure [190195]. For most FCC metals, the lowest energy facets are $\{111\},\{100\}$, and $\{110\}$, with the $\{111\}$ facet having the lowest surface energy and $\{110\}$ the highest. It should be mentioned that the Wulff theorem is only valid for NPs in a size regime where finite size effects and special undercoordinated sites (corners and edges) do not significantly alter the total surface energy [193]. Other phenomena that might result in a change of the surface energy of a given facet, such as strain [100; 196], surface reconstructions, and the presence of adsorbates or ligands [197-199], can also alter the shape of preferred structures.

Figure 9 shows the faceted shapes of Rh NPs supported on $\mathrm{MgO}(001)$ and bimetallic $\mathrm{Pt}-\mathrm{Au}$ NPs supported on $\mathrm{ZrO}_{2}$. The $\mathrm{Rh} \mathrm{NP}$ has a thin layer of oxide on its surface due to the exposure to oxygen $\left(2 \times 10^{-5}\right.$ mbar $)$ at $600 \mathrm{~K}$. It was shown that this NP shape could be described based on a Wulff construction if the oxide surface energies are considered. Such shape is different from that of a similar Rh NP after a reduction treatment [200]. In the case of the $\mathrm{Pt}_{0.8} \mathrm{Au}_{0.2} \mathrm{NP}$ shown in Figure 9(b), while the initial shape of the NP that was synthesized by the inverse micelle method was spherical, an annealing treatment at $500^{\circ} \mathrm{C}$ in air followed by $500^{\circ} \mathrm{C}$ in hydrogen allowed the NP to adopt a faceted shape following the Wulff structure [201].
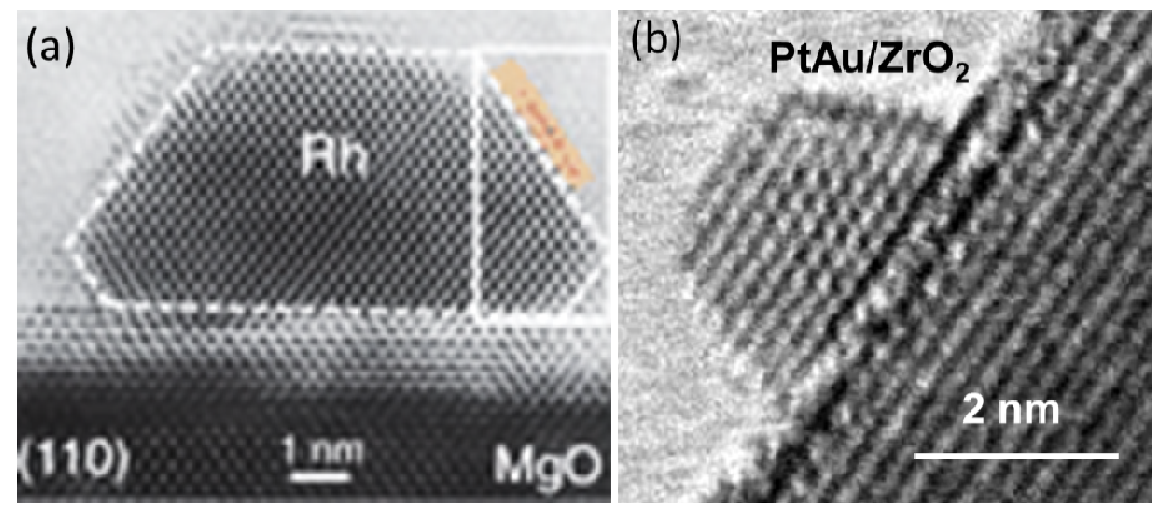

Figure 9: (a) HRTEM micrograph of a Rh particle on $\mathrm{MgO}(001)$ in (110) view [200]. (b) High resolution TEM image of a bimetallic $\mathrm{Pt}_{0.8} \mathrm{Au}_{0.2} \mathrm{NP}$ supported on a $\mathrm{ZrO}_{2}$ substrate annealed in air at $500^{\circ} \mathrm{C}$ for polymer removal and subsequent reduction in $\mathrm{H}_{2}$ at the same temperature [201]. Adapted from Refs. [200; 201].

One of the factors that can determine the shape of NPs is the epitaxial relationship between the NPs and the support. In fact, such relation dictates the interfacial plane orientation and by that, the overall shape of the NPs after high temperature annealing treatments $[32 ; 33 ; 100 ; 202]$. 
For instance, platinum NPs and strontium titanate (STO) supports are good candidates for this approach due to the very small lattice mismatch $(0.4 \%)$, which allows a good epitaxial relationship at the NP-support interface [32]. Iddir et al. used electron-beam lithography to pattern an epitaxial layer of Pt on different orientations of STO, leading to a lattice of Pt NPs with NP size of 30-100 $\mathrm{nm}$ [32]. Figure 10(a-c) shows the distinct NP shapes that were achieved by annealing the samples prepared on $\mathrm{STO}(100)$, $\mathrm{STO}(111)$ and $\mathrm{STO}(110)$ in nitrogen at $1177^{\circ} \mathrm{C}$ [33]. The overall shape of the NPs on each support was shown to follow the Wulff-Kaischew theorem $[189 ; 203]$, confirming the thermodynamic stability of the synthesized NPs [32].

While electron-beam lithography has been used in the past to generate NP catalysts with welldefined structures $[32 ; 33 ; 35]$, the cost and the limitation in the minimum NP size that can be achieved using this method makes it unsuitable for a large number of structure-sensitive catalytic processes. Once again, colloidal chemistry is a good alternative approach to prepare selfassembled arrays of size-selected and well-defined NPs [3; 14; 73; 100; 204-207]. Following the inverse micelle encapsulation synthesis method described in the previous section for the synthesis of size-selected NPs, shape control can also be achieved under certain environmental conditions (support, temperature, gaseous environment). For example, high temperature annealing of NPs in vacuum or under a reducing environment results in the formation of faceted NPs. For micellar NPs that are initially spherical, such faceted shapes are thermodynamically favored (free energy minimization), although they may not represent the global energy minima and might correspond to metastable states (local minima). Examples of such NP shapes are shown in Figure 10(d-f) [100]. 

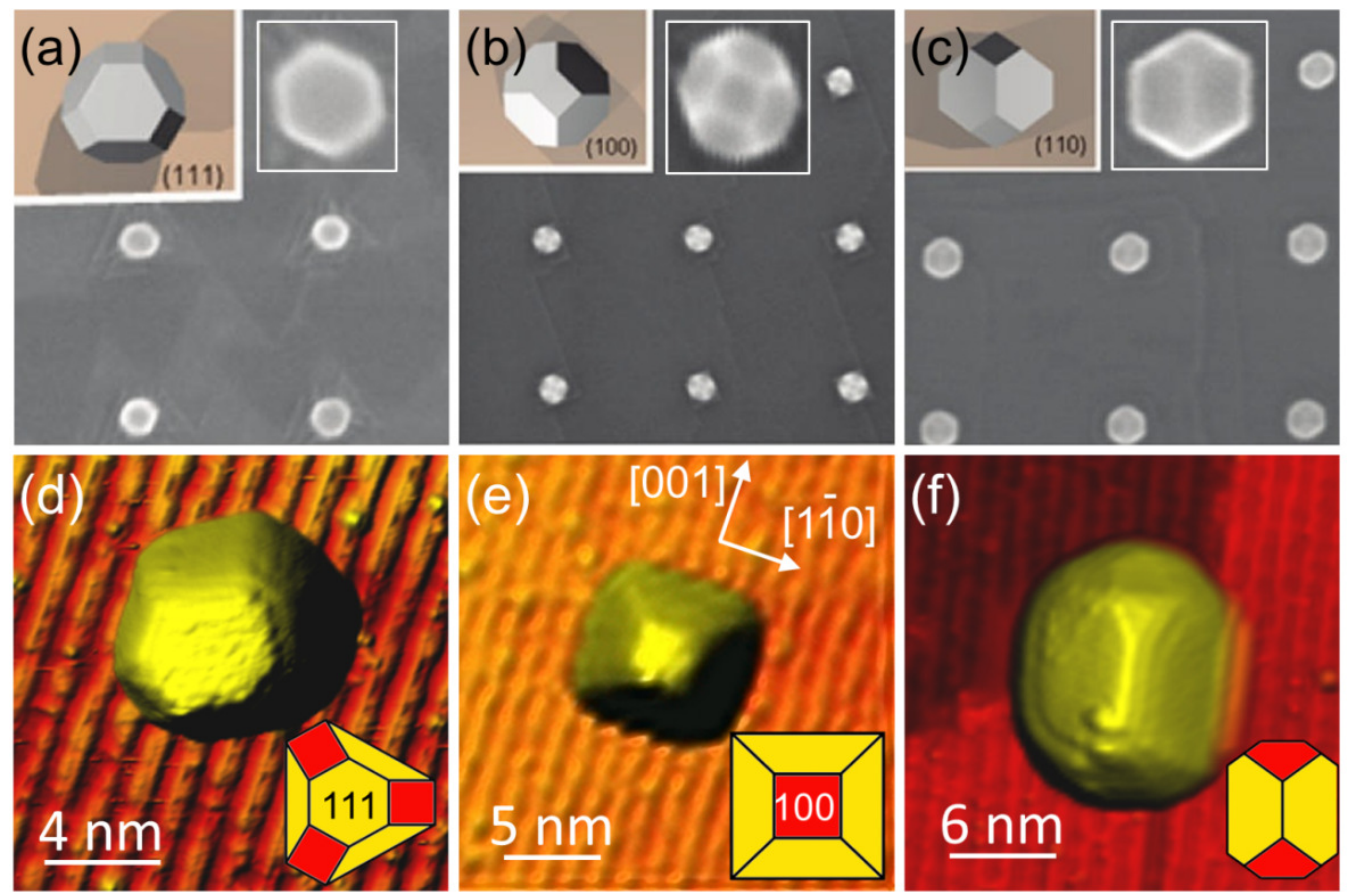

Figure 10 (a-c) SEM images of Pt NP arrays supported on STO(100), STO(110), and STO(111), respectively, acquired after annealing at $1177^{\circ} \mathrm{C}$. The NPs were arranged on $200 \mathrm{~nm}$ square lattices using e-beam lithography. The insets show the model shapes as well as a close-up of one of the NPs [33]. (d-f) STM images of the thermodynamically stable shapes of Pt NPs synthesized by inverse micelle encapsulation and supported on $\mathrm{TiO}_{2}(110)$. The images were acquired at room temperature after ligand removal and annealing in $\mathrm{UHV}\left(>1000^{\circ} \mathrm{C}\right)$. The inset cartoons show the model shapes with $\{100\}$ in red and $\{111\}$ facets in yellow [100]. In all images (a-f) the NP shape is thermodynamically stable and features only $\{111\}$ and $\{100\}$ facets. NPs in (a,d) have a $\{111\}$ facet at the interface, while those in $(b, e)$ have a $\{100\}$ facet, and those in $(c, f)$ a $\{110\}$ interfacial facet. Adapted from Refs. [33; 100].

\section{c) Evolution of the NP structure under reaction conditions}

Despite the initial well-defined NP structures described above, in many applications the NP catalysts should be viewed as dynamical entities subject to morphological, structural, as well as chemical changes under reaction conditions [208-212]. In other words, the carefully achieved kinetic shape and size of NPs may be lost under reaction conditions due to their tendency to progress toward their thermodynamically stable state. One example of such evolution that will not be discussed here are coarsening phenomena, in which the NPs grow in size to lower their surface energy [27; 143-149; 213].

Complicating matters is the fact that the thermodynamically favored NP structure is not unique, but is a distinct function of temperature, gas environment, reaction rate, and support conditions. Therefore, to unravel a correlation between the NP structure and its catalytic 
performance, the evolution of its geometry and chemical state should be monitored under operando conditions. To achieve this goal, there exists a variety of characterization techniques specifically designed to operate under real working catalytic conditions (operando measurements). These include synchrotron based x-ray spectroscopy, scattering, diffraction, and photoelectron emission techniques, as well as environmental electron microscopy and Fourier transformed infrared spectroscopy (FTIR) measurements. These techniques combined with first principle theoretical calculations under realistic environments may be used to gain in depth understanding of catalysts at work $[33 ; 209 ; 214-224]$. For any set of experimental conditions, certain measurement techniques may be especially suitable for monitoring the corresponding space- and time-dependent phenomena, depending on the specific length or time scale of interest. The ability to choose among such a variety of techniques provides a comprehensive toolbox to approach different problems [224]. Some examples of in situ and in operando catalyst characterization are described in this section.

Combining environmental measurement techniques can serve to elucidate the mechanism behind changes in a catalytic system under reaction conditions, which would otherwise be ambiguous. For example, using a combination of diffuse reflectance infrared spectroscopy (DRIFTS), $\mathrm{x}$-ray absorption spectroscopy (XAS), and mass spectrometry (MS), $\mathrm{Pd} / \mathrm{Al}_{2} \mathrm{O}_{3}$ catalysts ( $\sim 3 \mathrm{~nm}$ NPs) were studied during the catalytic reduction of NO by CO (CO/NO cycles) [225]. The fast variation in Pd-Pd coordination numbers obtained from EXAFS in alternating environments was initially assigned to a non-oxidative redispersion phenomenon [225]. However, in subsequent measurements, where time-resolved hard X-ray diffraction (HXRD) capabilities were available, a different scenario emerged [226]. The origin of the observed rapid variations in $\mathrm{Pd}-\mathrm{Pd}$ coordination numbers was discovered to be the dissociation of CO on Pd NPs during the CO cycle, resulting in the formation of $\mathrm{PdC}_{\mathrm{x}}$ species, which would rapidly turn back into metallic $\mathrm{Pd}$ during the NO cycle [226]. This is an example of how combining additional characterization techniques can provide a more reliable and accurate picture of the material system under study.

Figure 11 shows another example of how the presence of adsorbates can result in a NP shape transformation and weakening of the NP-support interaction [18; 19]. The shape of $\mathrm{Pt}_{13}$ clusters supported on $\gamma-\mathrm{Al}_{2} \mathrm{O}_{3}$ with different hydrogen coverages is shown in Fig. 11(a). By increasing the number of hydrogen atoms on the NP surface from 6 to 20, a shape transformation from a biplanar morphology (2D) to a cuboctahedron (3D) was theoretically predicted. Figure 11(b) 
compares the experimental results obtained by XANES and EXAFS analysis of $\mathrm{Pt}_{22} / \gamma-\mathrm{Al}_{2} \mathrm{O}_{3}$ [18] with the theoretical results (DFT) from $\mathrm{Pt}_{13} / \gamma-\mathrm{Al}_{2} \mathrm{O}_{3}$ [19]. An increase in first nearest neighbor coordination number (NN1) by increasing the hydrogen coverage demonstrates the transition of the NP shape from 2D to 3D [18]. The shape transition observed for the experimental $\mathrm{Pt}_{22} \mathrm{NPs}$ occurs at higher hydrogen coverage as compared to the model $\mathrm{Pt}_{13} \mathrm{NPs}$ due to their larger size. Figure 11(c) shows the variation in hydrogen coverage at room temperature as a function of the hydrogen pressure for the experimental $\mathrm{Pt}_{22}$ NPs [18] and theoretical $\mathrm{Pt}_{13}$ clusters [19]. In this

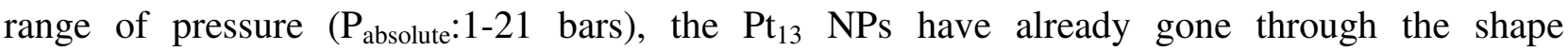
transformation and the hydrogen coverage is almost at its saturation level. However, slightly larger $\mathrm{Pt}_{22}$ NPs show a shape transition at around 7 bars. This example shows that the dynamic nature of NPs is a function of the NP size, the environment, and the support.
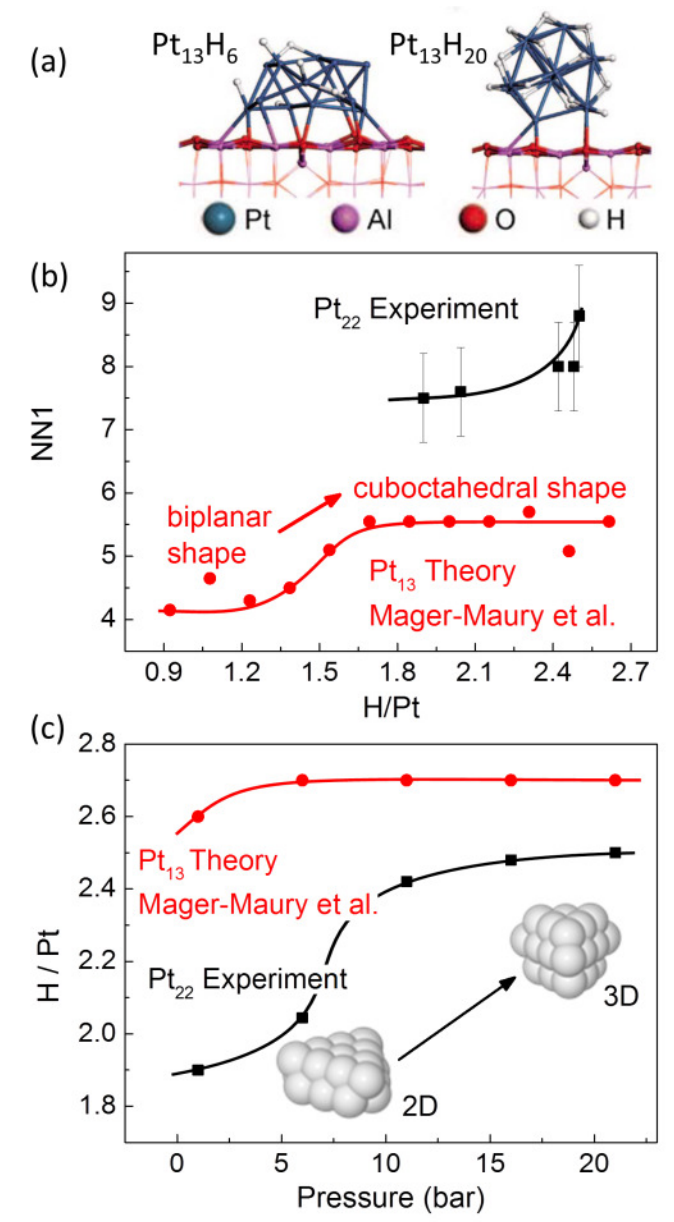

Figure 11: (a) Calculated structures for hydrogen-covered $\mathrm{Pt}_{13}$ clusters supported on the $\gamma$ $\mathrm{Al}_{2} \mathrm{O}_{3}(100)$ surface with 6 (bi-planar morphology) and 20 hydrogen atoms (cuboctahedron 
morphology) [19]. (b) Experimental Pt-Pt first nearest-neighbor coordination number (NN1) from in situ EXAFS analysis of $\mathrm{Pt} / \gamma-\mathrm{Al}_{2} \mathrm{O}_{3}$ catalysts as a function of the hydrogen coverage (black squares) and theoretical $\mathrm{NN1}$ for $\mathrm{Pt}_{13}$ from Mager-Maury et al. (red circles) [18]. (c) $\mathrm{H} / \mathrm{Pt}$ ratio calculated from the area of the $\triangle$ XANES spectra normalized by the corresponding area of large bulk-like Pt NPs measured at $183 \mathrm{~K}$ (black squares). For the large NPs used as reference in the normalization, a 1:1 $\mathrm{H} / \mathrm{Pt}$ ratio was considered. Model shapes for low and high hydrogen coverage are shown. Also plotted are theoretical $\mathrm{H} / \mathrm{Pt}$ ratios from Mager-Maury et al. for $\mathrm{Pt}_{13} \mathrm{NPs}_{\text {(red }}$ circles) [18]. Adapted from Refs. [18] and [19].

In order to understand the mechanism of induced shape transformations, the changes in the surface energy of different facets in the presence of adsorbates should be understood. In general, the presence of adsorbates reduces the surface energy [221]. Also, facets with higher surface energies correspond to higher adsorption energies [221]. The presence of adsorbates is expected to decrease the anisotropy of the surface energies of different facets, and therefore alter the NP shape toward more symmetrical shapes. Along the same lines, the growth of a certain facet might be favored in a given gas environment based on the binding energy of the adsorbate to that facet [227].

For instance, DFT calculations showed that $\mathrm{H}_{2}$ environments promote the stabilization of $\operatorname{Pt}(100)$, while CO environments favor $\operatorname{Pt}(210)$ facets [227]. The lower the binding energy of the adsorbate (e.g $\mathrm{N}_{2}$ on $\mathrm{Pt}$ ), the smaller will be its effect on the final NP shape [227]. The morphological changes induced by the chemisorption of adsorbates need to be taken into account when a model is being proposed to explain the reactivity of a catalyst, since the reaction environments may change the relative area of the most catalytically active surface facets/sites.

In addition, the presence of the adsorbates could reduce the energy barrier for metal atom selfdiffusion on the metal surfaces, resulting in surface roughening [228]. Figure 12(a,b) shows the changes in the surface morphology of an $\mathrm{Au}(111)$ single crystal upon exposure to $\mathrm{CO}$ adsorption-desorption cycles at room temperature. While the vacancy islands are hexagonal with straight and regular step edges on the clean surface, Figure 12(a), the CO exposed surface shows vacancy islands with rounded shape and irregular edges as well as small Au clusters on the upper terraces [Figure 12]. DFT calculation results are also shown in Figure 12(c,d). Depending on the atomic coordination numbers of the surface atoms, five different sites were considered [marked on Figure 12(c)]. The higher the reaction energy for each atomic position, the higher will be the energy barrier for that atom to climb to the upper terrace. However, as can be seen in Figure 12(d), the presence of $\mathrm{CO}$ on the surface drastically lowers the barrier energy for all considered sites. 

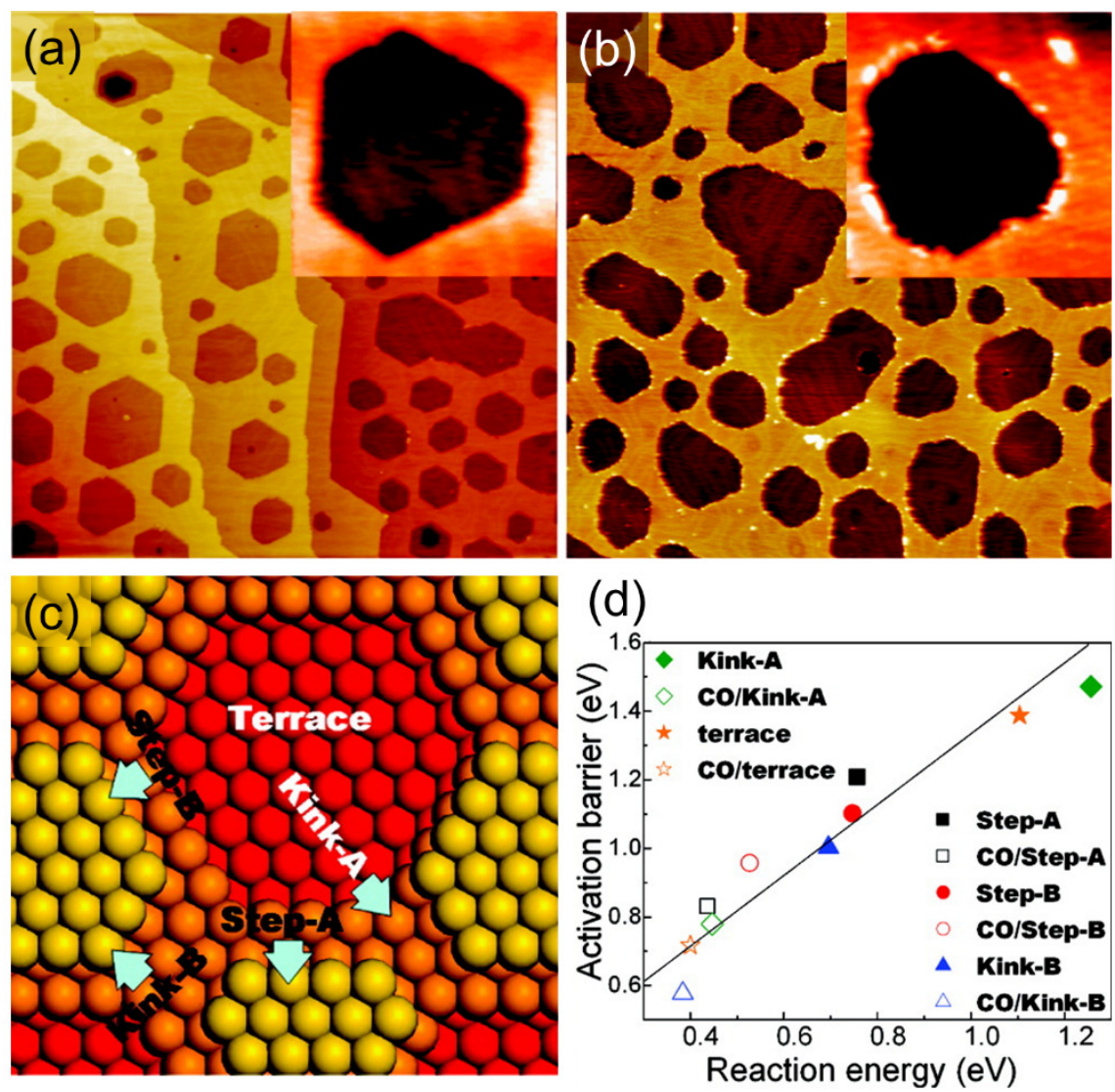

Figure 12: Room temperature STM images $(200 \mathrm{~nm} \times 200 \mathrm{~nm} ; 27 \mathrm{~nm} \times 27 \mathrm{~nm}$ inset $)$ of the vacancy island modified $\mathrm{Au}(111)$ surface before $\mathrm{CO}$ adsorption (a) and after a $\mathrm{CO}$ adsorption-desorption cycle (b). (c) Ball model of the modified $\mathrm{Au}(111)$ surface with five adsorption sites labeled. (d) Plot comparing the reaction energies vs reaction barriers for a climb of an $\mathrm{Au}$ atom from five different sites to the upper terrace before and after $\mathrm{CO}$ adsorption. Adapted from Ref. [228].

Another example of the adsorbate effect is given through DFT calculations demonstrating that the morphology and equilibrium shape of $\mathrm{Pd}$ and $\mathrm{Rh}$ nanocrystals can be modified under different $\mathrm{O}_{2}$ pressures, Figure 13 [221]. In vacuum, (111) and (100) facets are dominant, although smaller facets with higher Miller indexes were found to appear at corners. With increasing $\mathrm{O}_{2}$ pressure, oxygen adsorption was found to start on higher Miller index facets, stabilizing the otherwise unstable facets such as (110). By further increasing the $\mathrm{O}_{2}$ pressure, thin oxide layers start to form on the NP surface. For Ru NPs, the surface energy of oxide films on $\mathrm{Ru}(111), \mathrm{Ru}(100)$, and $\mathrm{Ru}(110)$ is nearly identical, resulting in the rounding of NPs featuring flat (111), (110) and (100) facets [221]. 

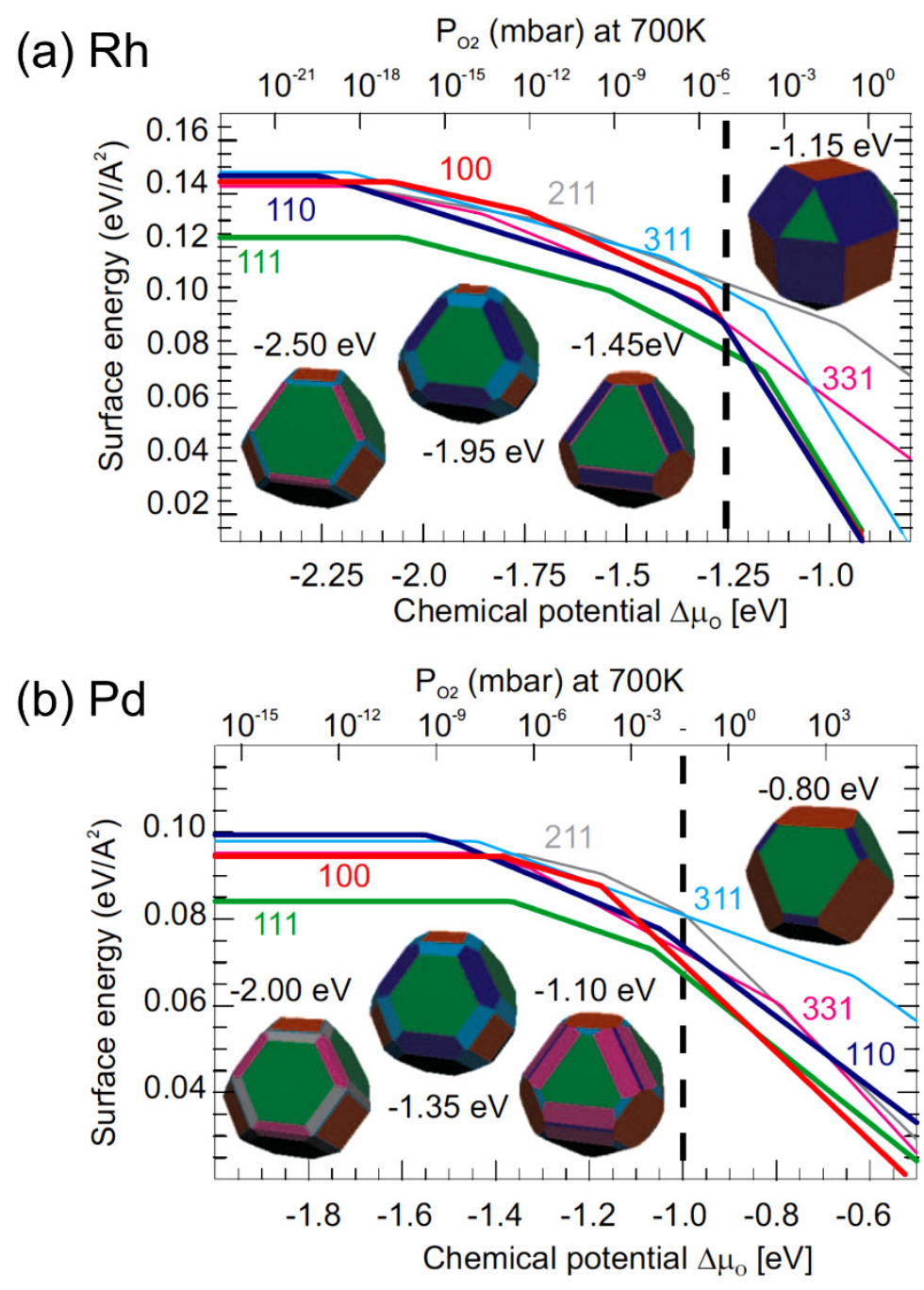

Figure 13: (a-b) Comparative phase diagram of oxygen adsorption on selected $\mathrm{Rh}$ and $\mathrm{Pd}$ nanocrystal surfaces: (111) green, (100) red, (110) blue, (311) light blue, (211) gray. The vertical lines in the phase diagrams are referring to the stability regime of the bulk oxides. Adapted from Ref. [221].

Lee et al. studied the equilibrium shape of Pt NPs at low oxygen pressure $\left(10^{-7}\right.$ Torr) after annealing at $1200^{\circ} \mathrm{C}$, finding spherical thermodynamically stable NP shapes with different facet orientations having similar surface energies [229]. On the other hand, NPs treated in inert environments such as vacuum were found to have a truncated cuboctahedron shape $[32 ; 100 ; 230$; 231].

Figure 14 provides several examples of the dynamic shape of NPs under different environments. Using grazing incidence small angle $\mathrm{x}$-ray scattering (GISAXS) and TEM measurements, the formation of thin surface oxides on $\mathrm{Ru}$ NPs supported on $\mathrm{MgO}(001)$ in oxygen 
environments $(600 \mathrm{~K})$ was shown to result in an increase in the total area of (100) facets at the expense of (111) surfaces, Figure 14(a). This change was found to be reversible during the subsequent NP reduction by CO [200]. For Pd NPs also supported on $\mathrm{MgO}(001)$, a similar reversible behavior was reported [Figure 14 (b-c)] using HR-TEM and the weak beam dark field technique (WBDF) [197].

Environmental measurement techniques can give insight into changes in catalyst structure during a reaction due to adsorbate interactions. An environmental high resolution TEM (HR-TEM) study by Giorgio et al. [232] showed that $\mathrm{TiO}_{2}$-supported 1-8 nm Au NPs adopt a faceted shape (truncated octahedra) under a $\mathrm{H}_{2}$ atmosphere, while they become rounded and de-wet the support under oxygen or CO oxidation reaction conditions, Figure $14(\mathrm{~d}, \mathrm{e})$. The rounding of the NPs was assigned to the presence of chemisorbed oxygen on the Au surface.

In addition, sample exposure to adsorbates might also change the support, and as a result, change the NP-support adhesion energy and the surface energy of different facets, which will in turn change the thermodynamic shape of the NPs. For example, using in situ TEM [Figure 14 (fh)], the evolution of the shape of Cu NPs was monitored under different gas environments [214]. For $\mathrm{Cu}$ NPs supported on $\mathrm{ZnO}$, a mixture of $\mathrm{CO}+\mathrm{H}_{2}$ was shown to reduce the $\mathrm{ZnO}$ support, resulting in higher adhesion energy and flattening of the NPs, Figure 14(h). Such effect was not observed on a non-reducible support such as silica [214].

The above discussion demonstrates the dynamic nature of the NP morphology under reactive environments, emphasizing the need for a thorough knowledge of not only the characteristic NP size, but also the most stable shape at a given thermodynamic condition, since both parameters will affect the chemical reactivity. We will discuss the effect of the NP size and shape on their catalytic performance in the next sections. 

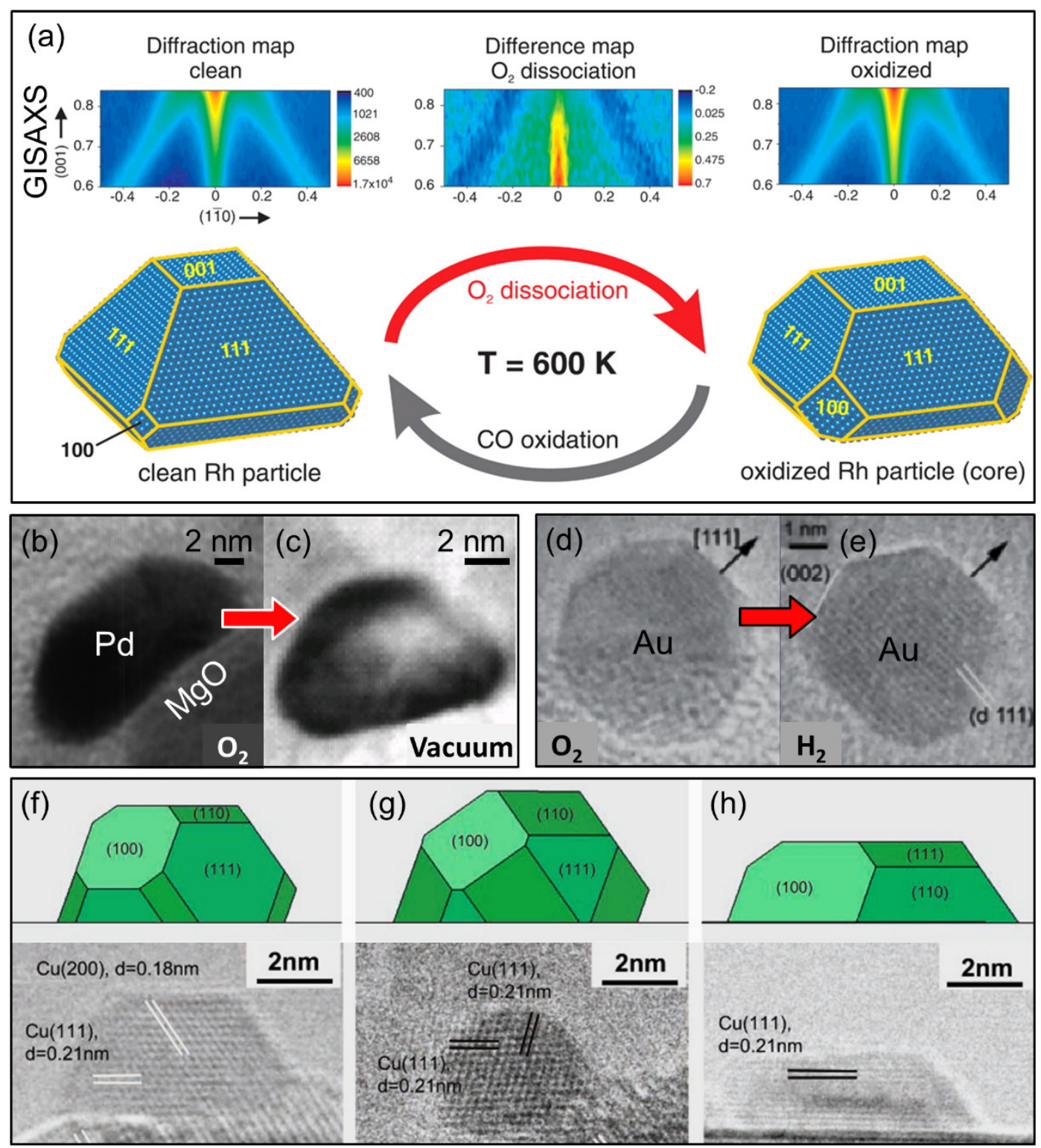

Figure 14: (a) (110) diffraction map of Rh NPs supported on $\mathrm{MgO}(001)$ measured by GISAXS at

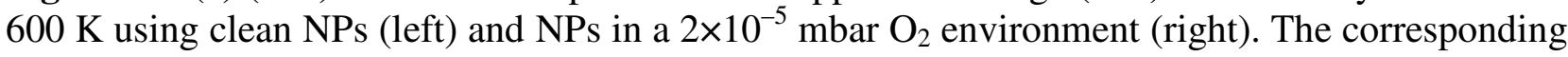
average model shapes are provided underneath the experimental data. The middle panel shows the difference map representing the dissociation of oxygen [200]. (b-c) TEM images of Pd NPs supported on $\mathrm{MgO}(001)$, annealed at $550^{\circ} \mathrm{C}$ in $10^{-3}$ Torr $\mathrm{O}_{2}$ (left), and in UHV (right). The Pd NPs were found to flatten under the oxygen environment [197]. (d-e) In situ evolution of the shape of $\mathrm{Au}$ NPs in $\mathrm{O}_{2}$ (left) and $\mathrm{H}_{2}$ (right) at a pressure of 2 mbar measured at room temperature with an environmental TEM. NP faceting in $\mathrm{H}_{2}$ and rounding in $\mathrm{O}_{2}$ environment is evident [232]. (f-h) In situ TEM images of a $\mathrm{Cu} / \mathrm{ZnO}$ catalyst in various gas environments together with the corresponding Wulff construction models (shown above) of the $\mathrm{Cu}$ nanocrystals at $220^{\circ} \mathrm{C}$ [214]. The images were recorded at a pressure of 1.5 mbar of (f) $\mathrm{H}_{2}$, and (g) a gas mixture of $\mathrm{H}_{2}$ and $\mathrm{H}_{2} \mathrm{O}$, $\left(\mathrm{H}_{2}: \mathrm{H}_{2} \mathrm{O}, 3: 1\right)$, and (h) in a mixture of $\mathrm{H}_{2}(95 \%)$ and $\mathrm{CO}(5 \%)$ at a total pressure of 5 mbar [214]. Adapted from Refs. [197; 200; 214; 232]. 


\section{Role of the nanoparticle size on catalytic activity and selectivity}

Nanoparticles or nanoclusters (NCs) are known to have drastically different physical and chemical properties as compared to their bulk counterparts, and in many cases greatly enhanced reactivities and selectivities have been reported for nanocatalysts $[14 ; 89 ; 96 ; 101 ; 190 ; 214-216$; 233-240]. Furthermore, the binding energies of many adsorbates on NPs are known to be sizedependent, with generally those on large NPs reaching a value close to that on extended single crystal surfaces, while small NPs may have smaller or larger binding energies [12; 112; 241-249].

Figure 15 shows examples of temperature programmed desorption (TPD) data demonstrating the size dependence in binding energy of adsorbates on supported metal NPs. A higher desorption temperature in TPD data generally corresponds to a higher binding energy of the adsorbate. The oxygen TPD data in Figure 15(a) are obtained from $\mathrm{TiO}_{2}(110)$-supported Au NPs and show a large difference in desorption temperature for the thin Au layer (2.3 layers $\rightarrow 645 \mathrm{~K}$ ) as compared to the thick layer ( $>6.0$ layers $\rightarrow 545 \mathrm{~K}$ ) [250]. A similar trend is observed for CO TPD data on Au NPs supported on $\mathrm{Al}_{2} \mathrm{O}_{3} / \mathrm{NiAl}(110)$ [Figure 15(b)], in which a decrease in desorption temperature from

$210 \mathrm{~K}$ to $160 \mathrm{~K}$ is seen by increasing the Au thickness from $0.15 \AA$ to $1.3 \AA$, after which a nearly constant desorption temperature is seen with increasing Au thickness [12]. Most TPD studies show an enhancement in binding energy of adsorbates with decreasing NP size [12; 250-256]. 

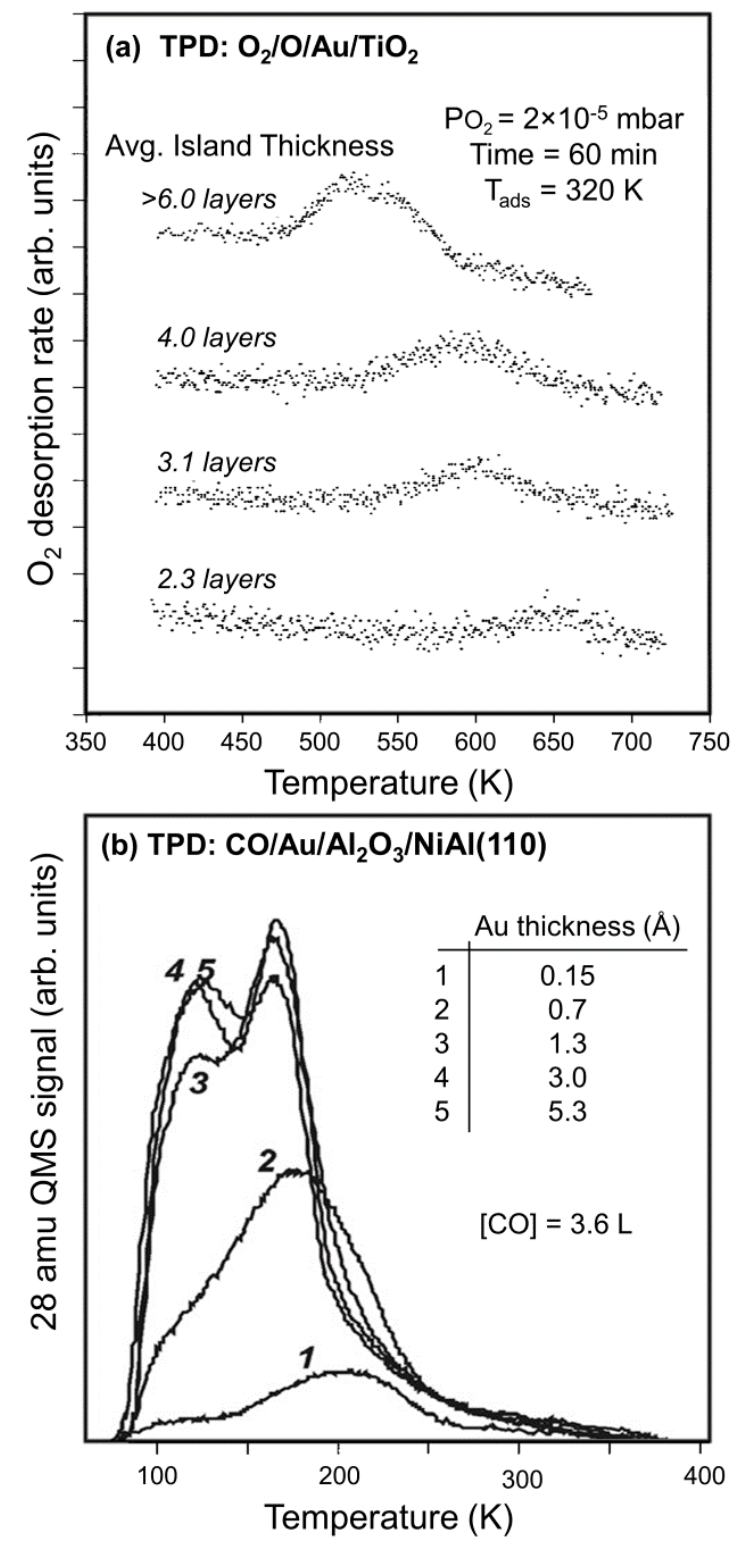

Figure 15: (a) $\mathrm{O}_{2}$ TPD curves corresponding to $0.35 \mathrm{ML}$ of oxygen adatoms adsorbed on $\mathrm{Au}$ islands with different average thicknesses (given in atomic layers). The atomic oxygen was dosed by introducing $2 \times 10^{-5} \mathrm{mbar}_{2}$ for $60 \mathrm{~min}$ through the hot filament of a mass spectrometer close to the sample surface. This was done since $\mathrm{O}_{2}$ does not readily dissociatively adsorb on gold. The $\mathrm{Au}$ islands covered the following fractions of the $\mathrm{TiO}_{2}(110)$ surface: $20 \%$ (2.3 layers), 38\% (3.1 layers), 36\% (4.0 layers), and 75\% (6.0 layers) [250]. (b) CO TPD spectra for gold islands deposited at $300 \mathrm{~K}$ on $\mathrm{Al}_{2} \mathrm{O}_{3} / \mathrm{NiAl}(110)$ as a function of $\mathrm{Au}$ coverage. $1 \mathrm{~L}$ (10 $\left.{ }^{-6} \mathrm{Torr} / \mathrm{s}\right)$ [12]. Adapted from Refs. [12; 250].

One approach to obtain quantitative values based on TPD measurements is the heating rate variation method (HRV) [257]. Following this approach, the TPD heating rate is varied and the 
changes in the TPD peak position are monitored to determine the desorption energy $\left(E_{d}\right)$ without making any assumptions concerning the pre-exponential factor or specific reaction mechanisms.

Figure 16(a) shows the desorption of $\mathrm{O}_{2}$ from $\mathrm{Au}$ NPs supported on $\mathrm{SiO}_{2} / \mathrm{Si}(111)$ for different heating rates $(\beta)$ [252]. The $\mathrm{Au}$ NPs were pre-oxidized using an $\mathrm{O}_{2}$-plasma treatment prior to the TPD and two sets of Au NPs with the average heights of $1.5 \mathrm{~nm}$ and $5 \mathrm{~nm}$ were investigated. Following the Falconer and Madix method [257], the slope in the plot of $\operatorname{Ln}\left(\tilde{T}_{\text {max }}^{2} / \tilde{\beta}\right)$ versus $1 / T_{\max }$ is equal to $E_{d} / k$, where $E_{d}$ is the desorption energy, and $k$ is the Boltzmann constant.
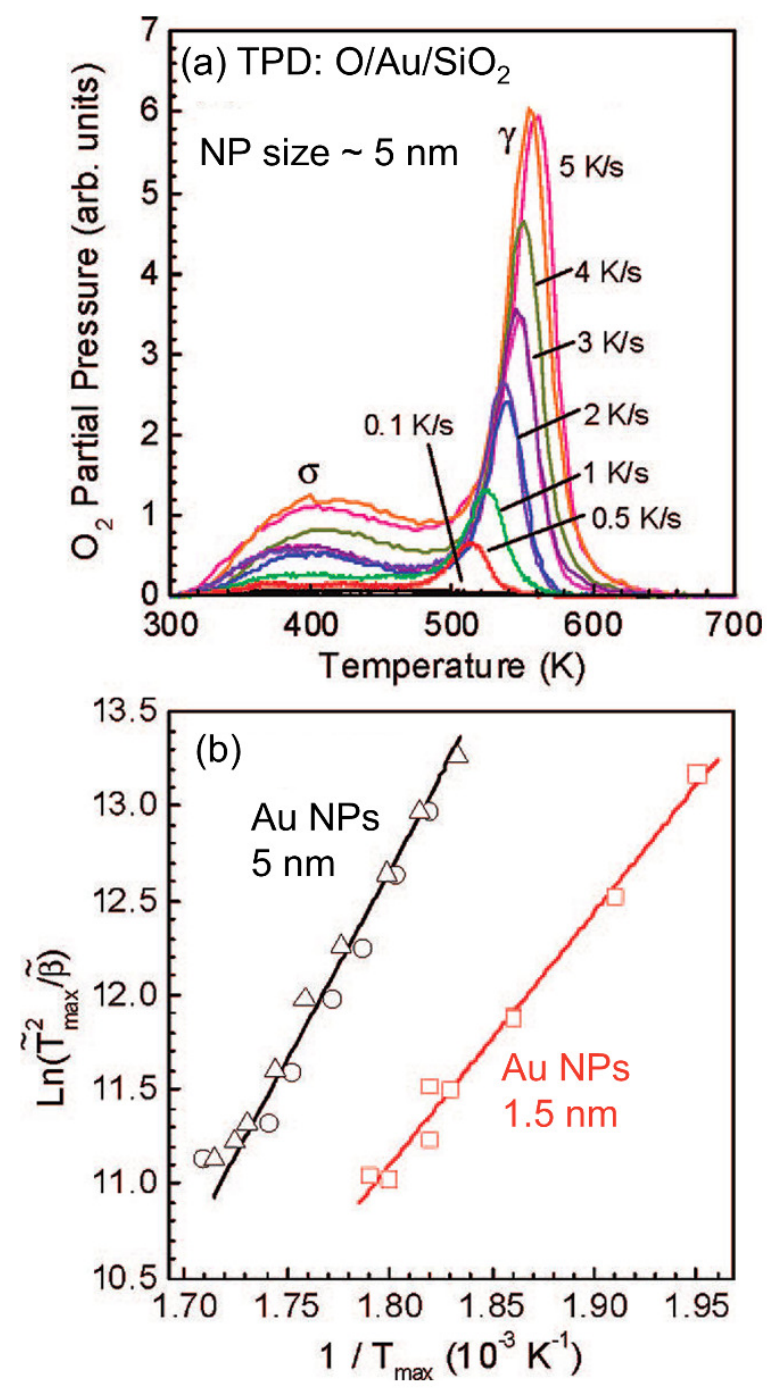

Figure 16: (a) Series of $\mathrm{O}_{2}$ TPD spectra obtained on $\sim 5 \mathrm{~nm}$ Au NPs deposited on $\mathrm{SiO}_{2} / \mathrm{Si}(111)$ for a constant initial oxygen coverage of $0.22 \mathrm{ML}$. The heating rate $(\beta)$ was varied from 0.1 to $5 \mathrm{~K} / \mathrm{s}$. (b) $\operatorname{Ln}\left(\tilde{T}_{\max }^{2} / \tilde{\beta}\right)$ versus $1 / \mathrm{T}_{\max }$ plots obtained from heating rate variation TPD data of $1.5 \mathrm{~nm}$ and $5 \mathrm{~nm} \mathrm{Au} \mathrm{NPs}$. The data correspond to the $\mathrm{O}_{2}$ desorption state labeled as $\gamma$ in panel (a). Adapted from Ref. [252]. 
From Figure 16(b), activation energies for $\mathrm{O}_{2}$ desorption of $1.2 \pm 0.1 \mathrm{eV}$ and $1.7 \pm 0.1 \mathrm{eV}$ were obtained for large $(\sim 5 \mathrm{~nm})$ and small $(\sim 1.5 \mathrm{~nm})$ Au NPs, showing a stronger binding of oxygen to smaller Au NPs [252].

For some catalytic systems, unravelling size-dependent trends in adsorbate binding strength can be highly complex, since factors other than the NP size can influence the results of the measurements. One such example is the adsorption of $\mathrm{CO}$ on palladium NPs. While several studies have shown an enhanced binding strength of CO on smaller NPs [258-261], other studies have found the opposite trend $[241 ; 243 ; 262-264]$.

To understand the difference between these apparently contradictory results we should take a closer look at the measurement techniques used to obtain the adsorption energy (i.e., TPD, microcalorimetry, and molecular beam experiments), as well as the effect of the NP support and presence of different adsorption sites. As compared to extended surfaces of single crystals, the larger variety of adsorption sites on NPs may result in a wider range of adsorption energies. Since the adsorbates tend to fill the sites with the highest binding energies first, the binding energies obtained at lower coverages are expected to be higher than those obtained when higher adsorbate coverages are considered. Usually, with increasing adsorbate coverage, repulsive adsorbateadsorbate interactions result in lower biding energies. Therefore, when comparing the results obtained from different characterization techniques, special attention should be paid to the adsorbate coverages employed. For instance, microcalorimetric data are often obtained for very low adsorbate coverages, allowing thus the investigation of the strongest binding sites. On the other hand, due to sensitivity-issues, TPD data on NPs are usually obtained for higher adsorbate coverages. Therefore, in order to compare the results obtained from these two techniques, a similar adsorbate coverage should be considered.

Figure 17 shows data obtained from different techniques regarding the adsorption energy of CO on Pd NPs. In Figure 17(a), the TPD data of alumina-supported Pd NPs show a small decrease in the TPD peak position from $503 \mathrm{~K}$ to $497 \mathrm{~K}$ by increasing the Pd thickness from $0.2 \AA$ to $3.5 \AA$ [254]. In Figure 17(b), the molecular beam study of CO desorption from Pd NPs supported on $\mathrm{MgO}(001)$ shows an enhanced desorption energy for NPs smaller than $5 \mathrm{~nm}$ [261]. Other molecular beam studies of $\mathrm{Pd} / \mathrm{mica}$ [259] and $\mathrm{Pd} / \mathrm{MgO}(100)$ [259-261] have reported similar results. Another calorimetric study on $\mathrm{Pd} / \mathrm{SiO}_{2}, \mathrm{Pd} / \eta-\mathrm{Al}_{2} \mathrm{O}_{3}$, and $\mathrm{Pd} / \mathrm{TiO}_{2}$ [258] also showed a higher binding energy of CO on smaller NPs. However, the calorimetric data in Figure 17(c) show 
the opposite trend for the $\mathrm{Pd} / \mathrm{Fe}_{3} \mathrm{O}_{4} / \mathrm{Pt}(111)$ system. The initial heat of adsorption of $\mathrm{CO}$ as a function of $\mathrm{Pd}$ coverage for the $\mathrm{Pd} / \mathrm{Fe}_{3} \mathrm{O}_{4}$ system was found to decrease with decreasing NP size [241; 243; 263; 264]. A different TPD measurement on $\mathrm{Pd} / \mathrm{Al}_{2} \mathrm{O}_{3}$ [262] also reveals a similar result to that from the calorimetric data.

In addition, another recent study has looked into the $\mathrm{CO}$ adsorption energy on $\mathrm{Pd} /$ $\mathrm{Al}_{2} \mathrm{O}_{3} / \mathrm{Ni}_{3} \mathrm{Al}(111)$ based on the residence time of $\mathrm{CO}$ molecules on Pd clusters, using a molecular beam technique [265]. This study also shows a decrease of the CO adsorption energy with decreasing clusters size down to $2 \mathrm{~nm}$, and a non-monotonic behavior for smaller NPs that are in the molecular regime. Although for similarly sized NPs the values obtained from this study are much smaller than those obtained from microcalorimetry measurements [243] (e.g. $30 \mathrm{~kJ} / \mathrm{mol}$ versus $110 \mathrm{~kJ} / \mathrm{mol}$ ), the general trend shows a good agreement.

An important phenomenon that should be taken into account in the former studies is $\mathrm{CO}$ disproportionation, in which the reduction of one $\mathrm{CO}$ molecule is accompanied by the oxidation of another [266; 267]. Such a reaction results in the production of $\mathrm{CO}_{2}$ and the contamination of the NP surface by the remaining carbon atoms. Although no CO disproportionation has been reported for $\operatorname{Pd}(100)$ or $\operatorname{Pd}(111)$ single crystals $[262 ; 266]$, the effect of carbon contamination on the heat of adsorption of $\mathrm{CO}$ on $\mathrm{Pd}(100)$ was investigated using single crystal adsorption calorimetry (SCAC) after carbon pre-dosing [266]. A decrease of $\sim 20 \mathrm{~kJ} / \mathrm{mol}$ in the initial CO heat of adsorption was observed for small C coverages of $0.05 \mathrm{ML}$, which could become as large as $120 \mathrm{~kJ} / \mathrm{mol}$ for higher $\mathrm{C}$ coverages of $0.2 \mathrm{ML}$. This observation suggests long range repulsive interactions between $\mathrm{C}$ atoms and CO molecules [266]. Interestingly, no carbon contamination due to CO dissociation was observed on $\mathrm{Pd} / \mathrm{MgO}(100)$ [259]. In the case of the $\mathrm{Al}_{2} \mathrm{O}_{3}$ support, large $\mathrm{Pd}$ NPs $(27 \mathrm{~nm})$ did not show any CO dissociation, while $2.5 \mathrm{~nm}$ Pd NPs showed significant carbon contamination and poisoning [262]. CO dissociation appears to be strongly affected by the NP treatments and the support properties. For instance, no CO dissociation was observed for Pd NPs deposited at high temperature on UHV-cleaved $\mathrm{MgO}(001)$, even on clusters as small as $2 \mathrm{~nm}$ [259], while Pd NPs smaller than $4 \mathrm{~nm}$ were reported to partially dissociate $\mathrm{CO}$ when supported on ex situ-cleaved $\mathrm{MgO}$ [267]. Such carbon contamination is shown to affect the low coordinated atoms and not the terrace sites, and might have to be considered as a possible explanation for the low adsorption energy observed on small Pd NPs supported on $\mathrm{Fe}_{3} \mathrm{O}_{4}$. 

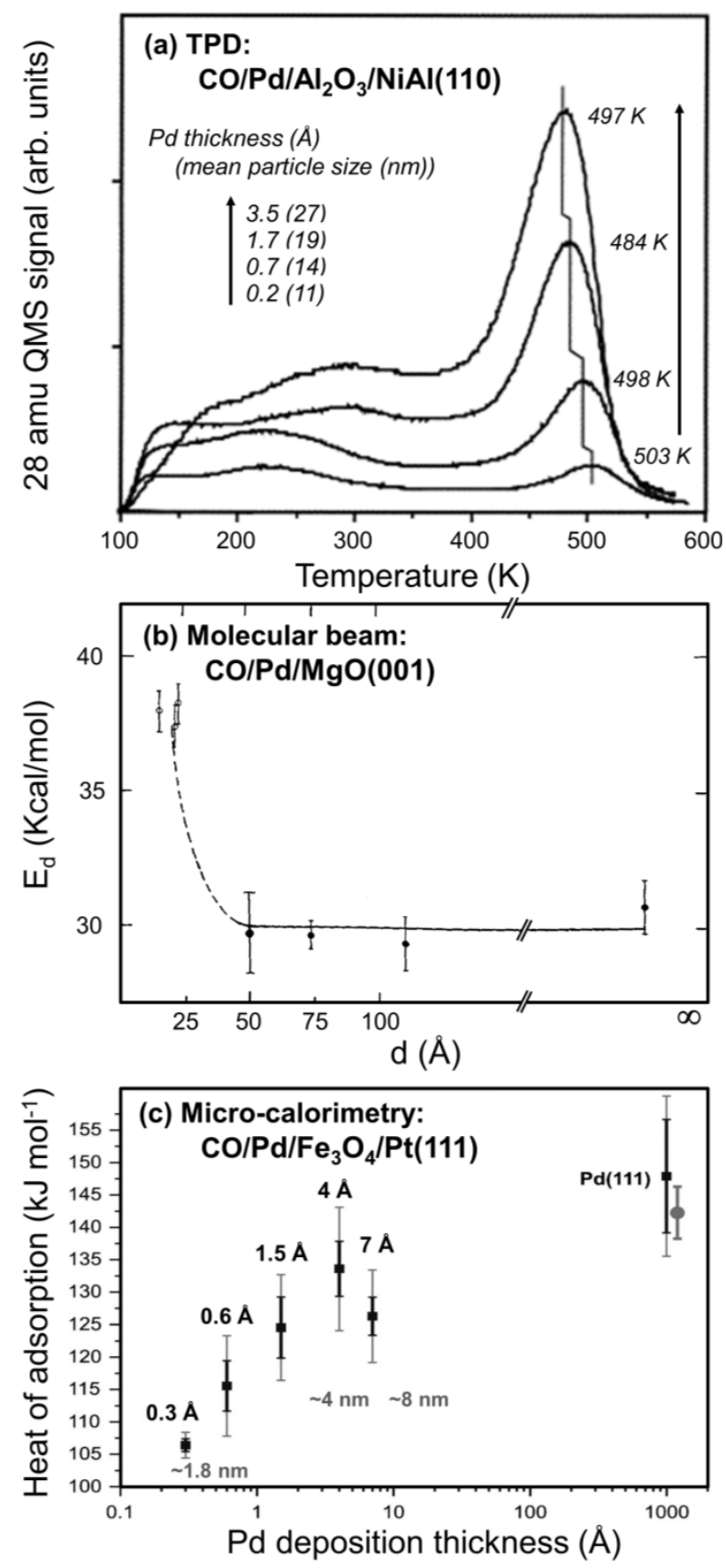

Figure 17: (a) CO TPD spectra of Pd NPs $\left(\mathrm{T}_{\mathrm{dep}}=90 \mathrm{~K}\right)$ pretreated with oxygen at $500 \mathrm{~K}$ and reduced with $\mathrm{CO}$ displayed as a function of the particle size. The samples were exposed to $2 \mathrm{~L}$ of $\mathrm{CO}$ at $100 \mathrm{~K}$ in each case [254]. (b) Variation of the initial desorption energy of CO as a function of the mean NP size. For the smallest NPs (open circles), the desorption energy is estimated from the nearly zero coverage value of the average adsorption life time [261]. (c) Initial heat of adsorption of $\mathrm{CO}$ as a function of $\mathrm{Pd}$ coverage on $\mathrm{Fe}_{3} \mathrm{O}_{4} / \mathrm{Pt}(111)$, with nominal $\mathrm{Pd}$ deposition thicknesses of $0.3,0.6,1.5,4$, and $7 \AA$, and also on the $\mathrm{Pd}(111)$ single-crystal surface [241]. The gray circle corresponds to a reference value of the $\mathrm{CO}$ heat of adsorption on $\mathrm{Pd}(111)$ from Ref. [268]. Error bars in (c): gray bars correspond to the standard deviation and black to the standard error of the mean [241]. Adapted from Refs. [241; 254; 261]. 
Another explanation that has been put forward for the opposite $\mathrm{CO}$ binding energy trend on $\mathrm{Pd} / \mathrm{Fe}_{3} \mathrm{O}_{4}$, namely, the decrease in the $\mathrm{CO}$ binding energy with decreasing NP size, is the effect of strain on the adsorption strength [243]. For instance, self-consistent DFT calculations for the adsorption of $\mathrm{O}$ and $\mathrm{CO}$ on flat and stepped $\mathrm{Au}(111)$ surfaces suggested that expansive strain would enhance the adsorbate binding energy [269]. Similarly, for the $\mathrm{Ru}(0001)$ surface, a lattice expansion was shown to increase the binding energies of $\mathrm{CO}$ and $\mathrm{O}$, while a lattice contraction would have the opposite trend [270]. Figure 18 shows the changes in the binding energy of $\mathrm{CO}$ and $\mathrm{O}$ as well that in the $\mathrm{CO}$ dissociation energy for the $\mathrm{Ru}(0001)$ surface due to strain effects.

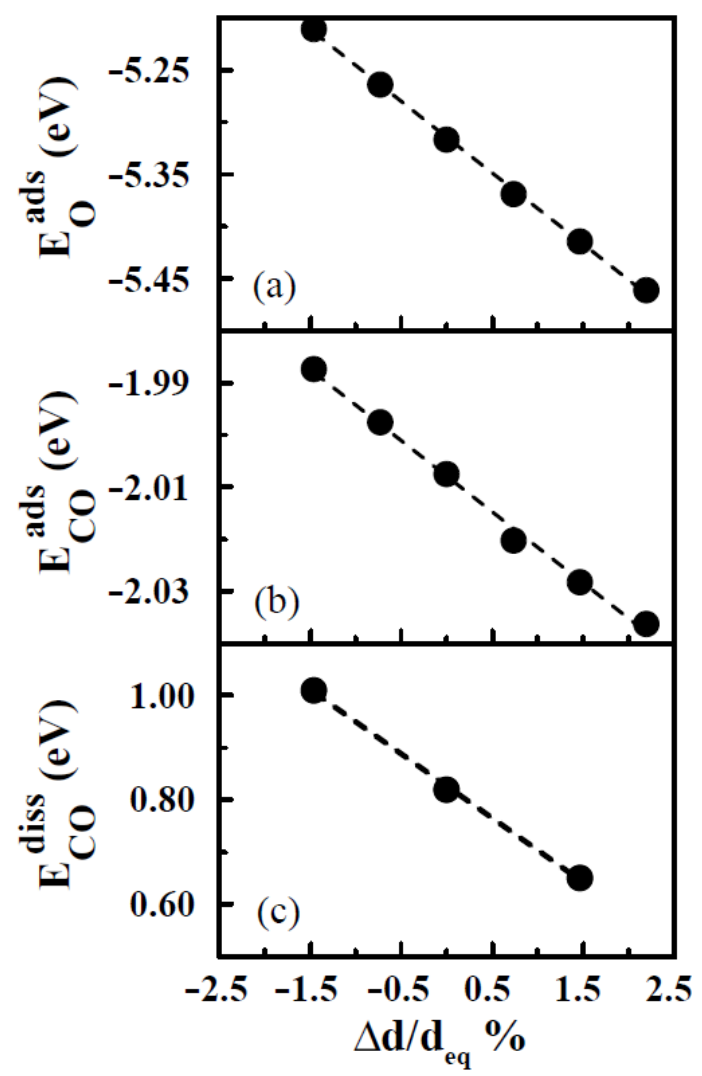

Figure 18: Effect of the relative change in the surface lattice constant $100 \times\left(\Delta d / d_{\text {eq }}\right)$ of a $\operatorname{Ru}(0001)$ surface on the (a) binding energy of atomic oxygen, (b) binding energy of molecular $\mathrm{CO}$, and (c) $\mathrm{CO}$ dissociation barrier relative to the clean surface plus a gas phase $\mathrm{CO}$ molecule as zero reference. $d_{\mathrm{eq}}$ is the calculated equilibrium lattice constant and $\Delta d$ is the change in the lattice constant as compared to the equilibrium value. Taken from Ref. [270].

A size dependent bond length contraction has been reported for metal NPs [271-278], while bond length expansion has been discussed for ionic compounds, including some metal oxide NPs [279-281]. 
Figure 19 shows the lattice contraction for (a) Au, and (b) Pd NPs as a function of the first nearest neighbor coordination number of the NPs and their reciprocal diameter, respectively.

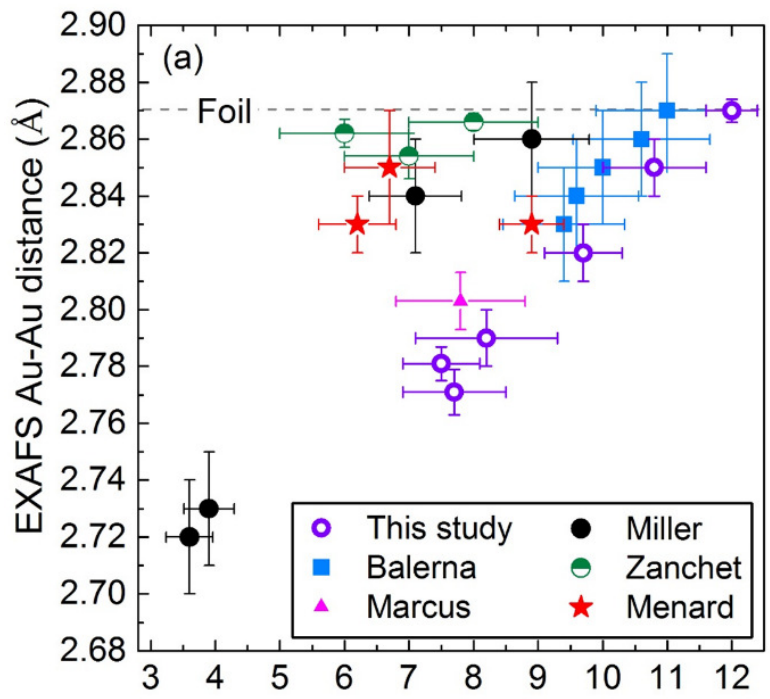

First nearest neighbor coordination number

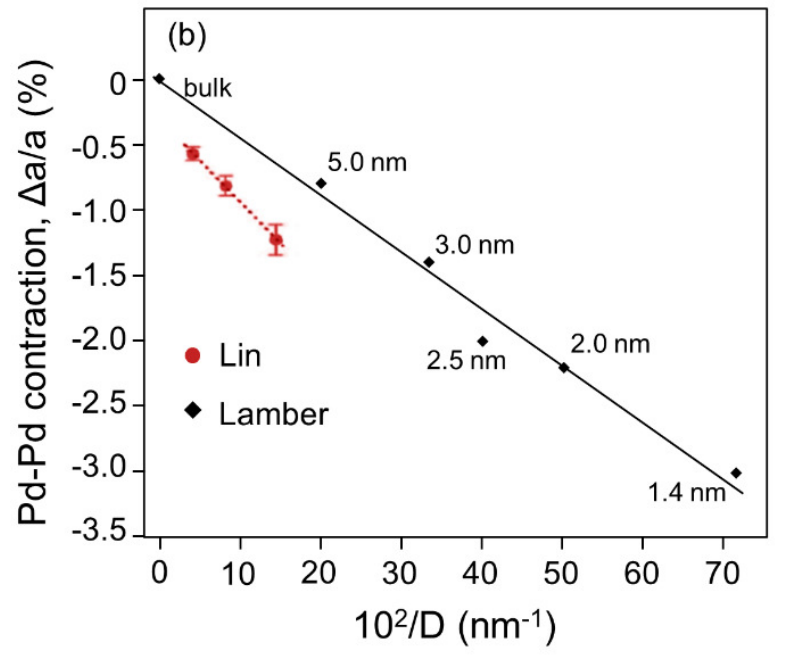

Figure 19: (a) Au-Au distance vs. $1^{\text {st }}$ nearest neighbor coordination number, obtained for Au NPs prepared via different synthesis methods [277; 282-286]. (b) Variation of the relative lattice parameter $\Delta \mathrm{a} / \mathrm{a}$ as a function of the reciprocal diameter of Pd clusters obtained from electron diffraction [287], and X-ray absorption spectroscopy measurements [278]. Adapted from Refs. [277; 278; 287].

Furthermore, similar to the case of extended surfaces, DFT calculations have shown that the lattice contraction induced by NP finite size effects could weaken the CO-Pd bond strength on small Pd NPs [245; 288].

Figure 20(a) shows several Pd NPs with one CO molecule deposited at the central 3-fold hollow site, $3 \mathrm{f}$ site, of each (111) facet. Although the $\mathrm{CO}$ adsorption energies are somewhat 
scattered, a trend could be observed for the strongest binding sites of a given NP size. In the small size regime there is an increase in adsorption energy with decreasing NP size, but in the larger size-regime, the trend is reversed [245].
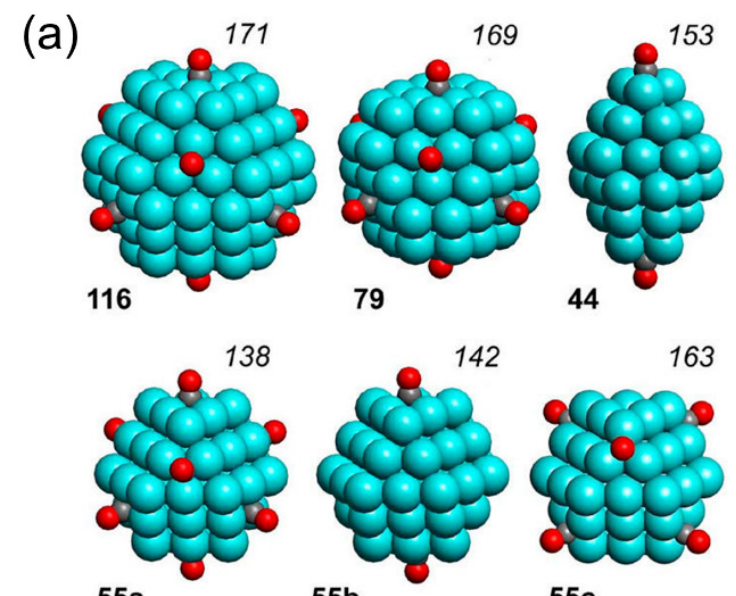

$55 a$

$55 b$

$55 \mathrm{c}$
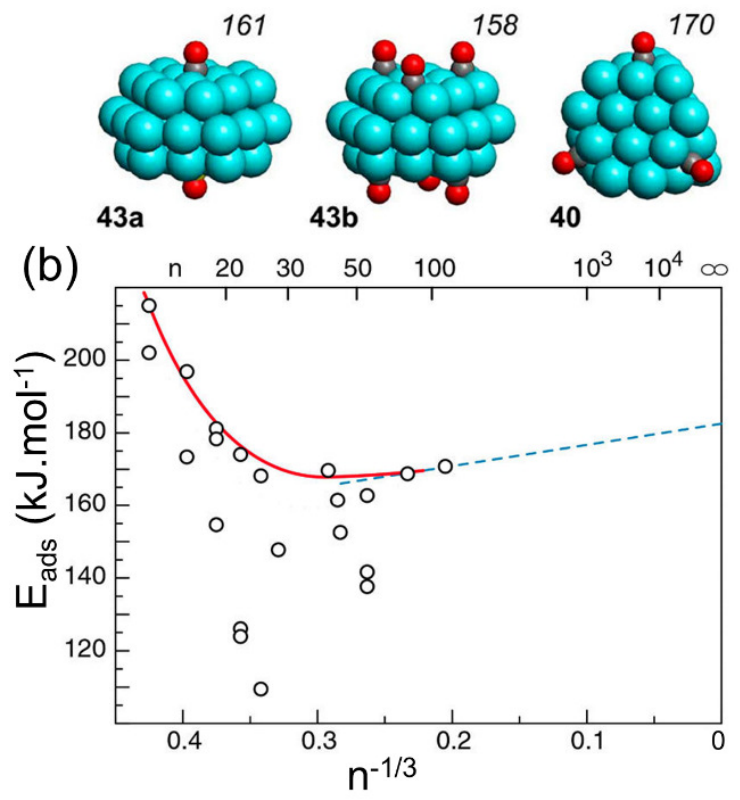

Figure 20: (a) Nanoclusters with $\mathrm{CO}$ adsorbates: $\mathrm{Pd}_{116}\left(\mathrm{O}_{\mathrm{h}}\right.$ symmetry), $\mathrm{Pd}_{79}, \mathrm{Pd}_{55}\left(\mathrm{O}_{\mathrm{h}}\right.$ for $55 \mathrm{a}$ and $\mathrm{D}_{3 \mathrm{~d}}$ for $55 \mathrm{~b}$ and $\left.55 \mathrm{c}\right), \mathrm{Pd}_{43}\left(\mathrm{D}_{3 \mathrm{~d}}\right), \mathrm{Pd}_{44}$, and $\mathrm{Pd}_{40}\left(\mathrm{~T}_{\mathrm{d}}\right)$. Calculated adsorption energies (italic) in $\mathrm{kJ} . \mathrm{mol}^{-1}$. Eight, six/two, and four adsorbed CO molecules per cluster for models with $\mathrm{O}_{\mathrm{h}}, \mathrm{D}_{3 \mathrm{~d}}$, and $\mathrm{T}_{\mathrm{d}}$ symmetry, respectively. (b) DFT calculated energies of $\mathrm{CO}$ adsorption, $\mathrm{E}_{\mathrm{ads}}$ (open circles, kJ $\mathrm{mol}^{-1}$ ), on $\mathrm{Pd}_{\mathrm{n}}$ clusters with $\mathrm{n}$ from 13 to 116 (some shown in panel (a)) as a function of $\mathrm{n}^{-1 / 3}$. The values for the most stable adsorption complexes (for a given nuclearity $n$ ) are approximated by the solid line. The scaling trend extrapolated to the limit of an infinite (111) surface is shown as a dashed line [288]. Adapted from Ref. [245]. 
However, the above findings do not necessarily imply that the $\mathrm{CO}$ molecules should have a lower binding energy to smaller NPs, since other concurrent effects must be considered as well. In fact, in the above theoretical comparison of the binding energy of CO to NPs with different sizes, the CO molecules were attached to similar Pd configurations (e.g. 3-fold sites on (111) facets of the NPs or bridge sites at the edges). Therefore, although the on-top sites on small NPs may adsorb CO slightly weaker, it cannot be disregarded that such NPs would also have a significant higher population of low coordinated atoms at corners and edges. Since low coordinated sites are known to display stronger $\mathrm{CO}$ binding [289], the average binding energy of $\mathrm{CO}$ on the whole NP, which is the parameter measured in the ensemble-averaging calorimetry studies, should still be stronger for smaller NPs. In addition, we should have in mind that in calorimetry measurements the $\mathrm{CO}$ coverages are kept very low in order to only populate the sites with the strongest adsorption energies. Therefore, $\operatorname{Pd}(111)$ facets which have the highest coordination number (and therefore lowest $E_{a d}$ ) are most likely the last sites to be occupied by $\mathrm{CO}$ molecules, and therefore, the theoretical data presented in Figure 20 should not be compared directly to the calorimetric data.

Here we need to mention that the most plausible explanation for the $\mathrm{CO}$ binding energy trend observed in the case of $\mathrm{Pd} / \mathrm{Fe}_{3} \mathrm{O}_{4}$ might be a support-related phenomenon. The presence of surface defects (e.g. small $\mathrm{Fe}$ or $\mathrm{FeO}_{\mathrm{x}}$ islands) or partial encapsulation of the $\mathrm{Pd} / \mathrm{Fe}_{3} \mathrm{O}_{4}$ system would stabilize the smaller NPs and weaken the adsorbate binding through the stronger NP-support interaction [147]. Direct evidence for the SMSI effect and Pd encapsulation by iron oxide has been reported recently, confirming the possibility of the important role played by the support on the binding of adsorbates to small Pd NPs on $\mathrm{Fe}_{3} \mathrm{O}_{4}$ [290]. Additional support-related phenomena will be discussed in more detail in section 3.3.

Calorimetric measurements of oxygen adsorption on $\mathrm{Pd} / \mathrm{Fe}_{3} \mathrm{O}_{4}$ have also shown a non-uniform dependence on NP size, namely, for NPs smaller than $4 \mathrm{~nm}$ the oxygen binding energy increases with increasing NP size, while for NPs larger than $4 \mathrm{~nm}$ the reverse trend is observed. For large Pd NPs the adsorption energy decreases until it approaches the value of the $\operatorname{Pd}(111)$ surface [243]. In agreement with calorimetric measurements, TPD data of oxygen chemisorption on $\sim 4.5 \mathrm{~nm} \mathrm{Pd}$ NPs supported on $\mathrm{SiO}_{2} / \mathrm{Si}(111)$ evidenced a desorption peak temperature $(940 \mathrm{~K})$ larger than that on $\operatorname{Pd}(111)(810 \mathrm{~K})$, indicating stronger oxygen binding for the NPs [243], although for smaller NPs TPD data revealed a further increase in the binding energy of oxygen with decreasing NP size, Figure 21. 


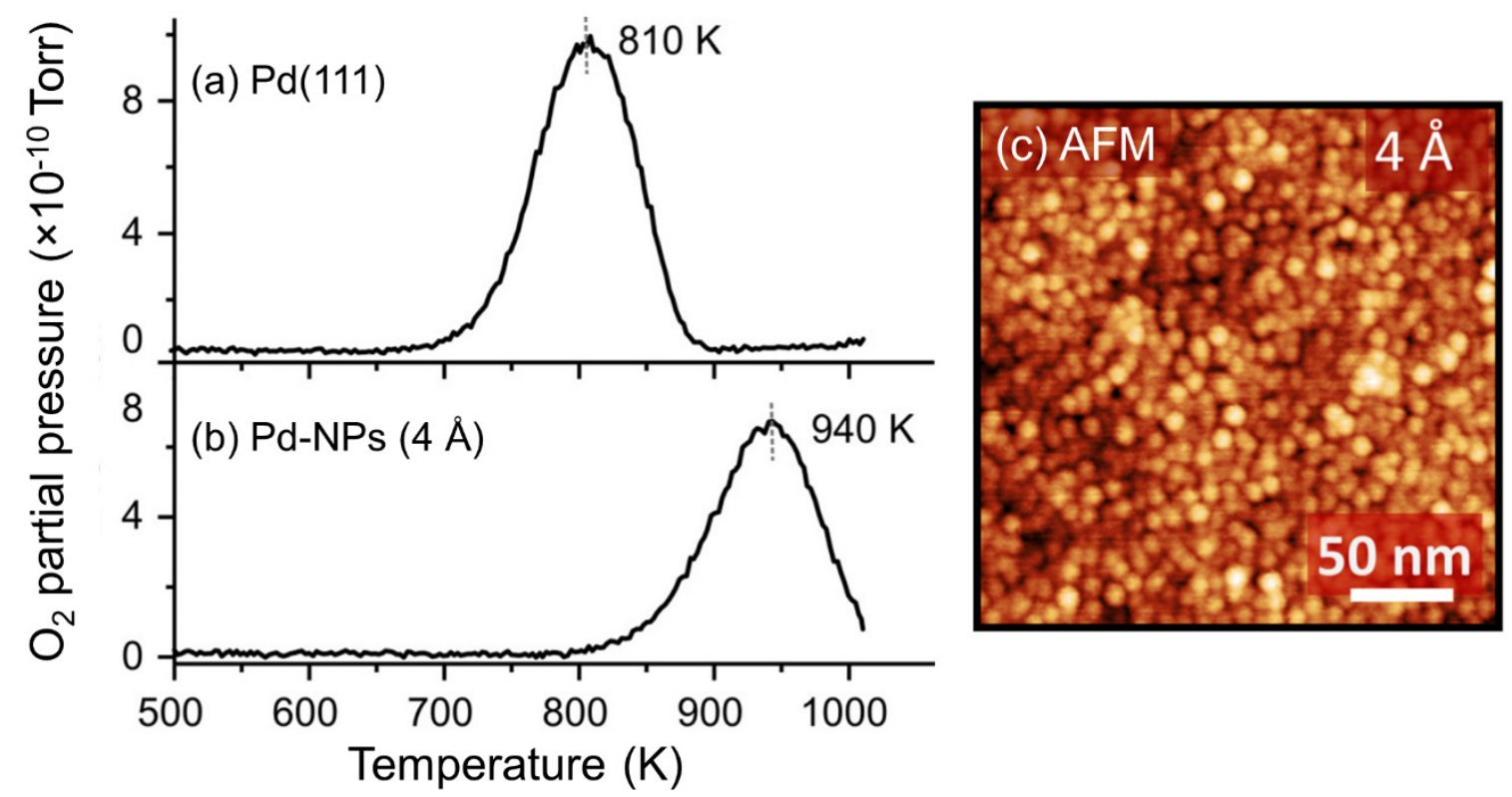

Figure 21: $\mathrm{O}_{2}$-TPD spectra from (a) Pd(111) and (b) PVD-deposited Pd NPs supported on $\mathrm{SiO}_{2} / \mathrm{Si}(111)$ with a nominal Pd deposition thickness of $4 \AA$ (average particle size $\sim 4.5 \mathrm{~nm}$ ), with the corresponding AFM image shown in (c). The signals correspond to desorption of $\sim 0.1 \times 10^{15} \mathrm{O}$ atoms $\cdot \mathrm{cm}^{-2}$. Adapted from Ref. [243].

For a number of catalytic reactions, the reactivity of NPs also shows a size dependence, with the NP size affecting the reaction rate as well as the selectivity. The surface to bulk ratio of NPs, assuming a similar shape, follows a 1/r behavior, and therefore, smaller NPs have a higher percentage of their atoms on the surface. This means that in order to obtain improved catalytic reactivities for samples made with similar metal/support loading, the synthesis of smaller NPs is preferred to ensure that a high metal surface area is available for reaction. This will be particularly important in case of noble metals such as $\mathrm{Pt}, \mathrm{Pd}$ and $\mathrm{Au}$, for which the high cost of the metals puts a greater constraint on the design of the catalysts.

The term "size-effect", however, does not simply refer to the amount of available catalyst surface area, but rather deals with how the performance of each surface atom is affected by the size of the NPs. Therefore, to study size-effects, it is common practice to normalize the activity by the available surface area (e.g. obtained from chemisorption measurements) to ensure that a comparison of different samples is made based on their performance per unit of available surface. There are several ways to normalize the activity, with the most widely used approach being the calculation of the "turnover frequency" (TOF). In the forthcoming sections, the comparison 
between the activities of different systems will be based on catalytic performance data normalized by the surface area of the NPs. However, we should warn that in certain cases the above approach might be misleading in evaluating the merit of a catalyst. This issue occurs when either there is no significant size-dependence, or a better performance in terms of TOF is obtained for the larger NPs. Since the total activity (TOF $\times$ surface area) is the ultimate figure of merit for a catalyst, the higher surface area of the smaller NPs may compensate their lower TOF, leading to a better performance.

Another issue that should be considered cautiously is the definition of the "size" in different studies. There is no universal definition of NP size, and hence, it may conveniently vary based on the measurement techniques used. For instance, scanning probe microscopy techniques such as STM and AFM are known to provide reliable measurements of the NP height, but not its diameter. SPM images are based on a convolution of the tip shape and the surface morphology, resulting in an overestimation of the NP diameter. On the other hand, TEM-high-angle annular dark-field scanning transmission electron microscopy (HAADF-STEM) techniques usually measure the diameter of NPs. In some cases, cross sectional TEM measurements enable a better understanding of the NP aspect ratio, but, nonetheless, the term "size" in most cases applies to one characteristic dimension of the NPs, such as their height or diameter. In contrast, DFT studies usually define the NP size based on the number of atoms (e.g. volume). Therefore, care should be taken while comparing size-effects in studies that are based on different measurement/calculation techniques.

The effect of NP size on the catalytic activity of NPs appears to be complex. Catalytic reactions could be divided into two categories: structure-sensitive and structure-insensitive [291]. In structure insensitive reactions, the reaction rate per surface area of the catalyst (e.g. TOF) is largely independent of the NP size, shape, metal loading, and dispersion, and in the case of extended surfaces is independent of the $\{\mathrm{hkl}\}$ single crystal orientation. However, care should be exerted when dealing with structure insensitive reactions since several artifacts could result in such insensitivity. For instance, in nanoscale catalysts, if the NP size in the differently prepared catalysts is larger than the threshold of non-bulk behavior, no size-effect is to be expected. Another concern is the lack of a sufficiently narrow size and shape distribution, which results in an ensemble averaging of size- and shape-dependent properties which could smear out the structure sensitive signatures. 
The structure sensitivity depends both on the NP material and the reaction itself. For instance, for Pt catalyst, reactions such as ethylene hydrogenation [292-294] and the water gas shift reaction [295; 296] are suggested to be structure-insensitive, while benzene hydrogenation [297], pyrrole hydrogenation [294; 298], ethane hydrogenolysis [292; 299], cyclohexene dehydrogenation [293; $300]$, the oxidation of alcohols $[3 ; 114 ; 291]$ and other oxidation reactions $[16 ; 301]$ are generally structure dependent. Adding to this complexity, the reaction conditions can also change the structure sensitivity trends, as is the case for the hydrogenation of cyclohexene [302]. At low pressure $\left(\sim 10^{-7}\right.$ Torr), benzene is the main reaction product when working in excess of hydrogen over $\operatorname{Pt}(223)$ stepped surfaces $\left(25-150{ }^{\circ} \mathrm{C}\right)$, while at higher pressure $\left(\sim 10^{2}\right.$ Torr $)$, cyclohexane production dominates [302]. While the low-pressure reactions proceed on the clean metal surface and are structure sensitive, the high-pressure reaction is apparently structure insensitive [302].

Figure 22 shows two examples of structure-insensitive reactions over Pt NPs. The TOF values for the water-gas shift reaction over a variety of $\mathrm{Pt} / \mathrm{TiO}_{2}$ catalysts (with sizes ranging between 1.2 $\mathrm{nm}$ and $16.2 \mathrm{~nm}$ ) are shown in Figure 22(a). Not only is the TOF similar for different catalysts, but also the activation energies are identical [296]. Similarly, Figure 22(b) shows that the activity of differently-shaped (wires, NPs, etc.) Pt catalysts with distinct metal loading is structure insensitive for the hydrogenation of ethylene [303]. 
(a) $\mathrm{CO}+\mathrm{H}_{2} \mathrm{O} \rightarrow \mathrm{CO}_{2}+\mathrm{H}_{2}$

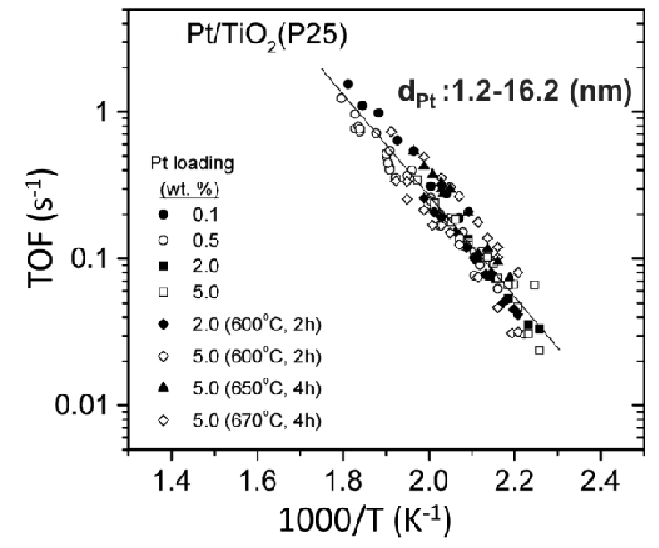

(b) $\mathrm{C}_{2} \mathrm{H}_{4}+\mathrm{H}_{2} \rightarrow \mathrm{C}_{2} \mathrm{H}_{6}$

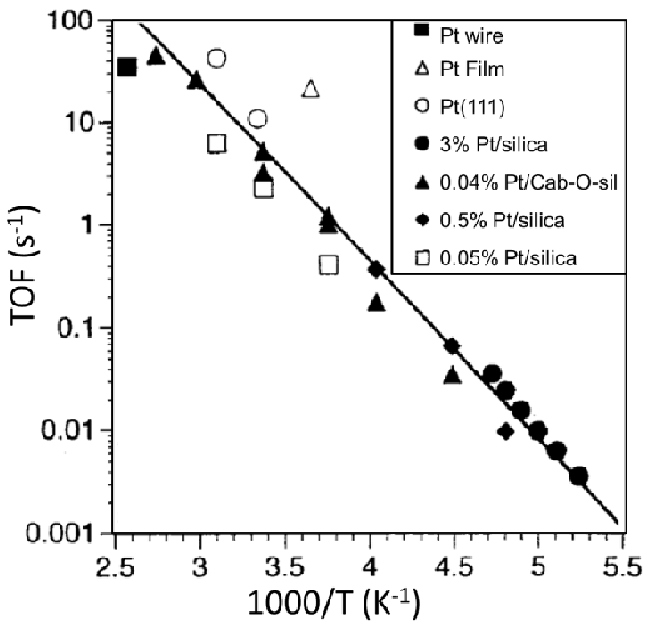

Figure 22: (a) Arrhenius plot of turnover frequencies (TOF) of $\mathrm{CO}$ conversion in the water gas shift reaction over Pt NP/TiO 2 catalysts with sizes ranging between $1.2 \mathrm{~nm}$ and $16.2 \mathrm{~nm}$ [296]. (b) Arrhenius plot of TOF for ethylene hydrogenation on differently sized/shaped Pt catalysts corrected for similar reaction conditions [303].

Now let's have a closer look at the origin of the structure insensitivity in some chemical reactions. In the case of the water-gas shift reaction over platinum (Figure 22a), a detailed study of the reaction kinetics was carried out by Stamatakis et al. using a combination of DFT calculations and kinetic Monte Carlo (KMC) simulations [304]. The energy barriers and pre-exponential factors were calculated for all elementary steps on different Pt surfaces, and the results were fed into the KMC simulation. Following this approach it was found that for high $\mathrm{CO} / \mathrm{H}_{2} \mathrm{O}$ ratios (e.g. a ratio of 0.5 similar to that used in Figure 22a), the reaction is structure insensitive, while for low ratios (e.g. ratio of $10^{-3}$ ), the step edges are much more active than the terrace sites. Therefore, the structure sensitivity reported by some authors for this reaction was shown to have a non-intrinsic 
character, but could be tuned depending on the reaction conditions. The main reason for this difference in structure sensitivity was found to be the different reaction pathways underwent when distinct $\mathrm{CO} / \mathrm{H}_{2} \mathrm{O}$ ratios are considered: while at high $\mathrm{CO} / \mathrm{H}_{2} \mathrm{O}$ ratios the carboxyl pathway is dominant, at low ratios the WGS reaction proceeds mainly through full $\mathrm{H}_{2} \mathrm{O}$ dissociation and direct $\mathrm{CO}$ oxidation [304]. At low reactant ratios and high enough temperature (e.g. $570 \mathrm{~K}$ ), both, $\mathrm{CO}$ and atomic oxygen were present at step sites. However, under high $\mathrm{CO} / \mathrm{H}_{2} \mathrm{O}$ ratios such as those shown in Figure 22a, the step sites in $\mathrm{Pt}(211)$ were found to be completely poisoned by $\mathrm{CO}$ and therefore, some elementary steps were entirely prohibited at step sites (e.g. $\mathrm{H}_{2} \mathrm{O}$ dissociation at steps). Although the $\mathrm{CO}$ bond to a step site could still contribute in other reaction stages, the $\mathrm{CO}$ poisoning of the step sites could be held responsible for the structure insensitivity of Pt catalysts at high $\mathrm{CO} / \mathrm{H}_{2} \mathrm{O}$ ratios (e.g. 0.5) [304].

In the case of the ethylene hydrogenation reaction, another type of poisoning seems to be the origin of the structure insensitivity [293; 305-307]. The concentration of different adsorbate species on $\operatorname{Pt}(111)$ and $\operatorname{Pt}(100)$ surfaces was measured using sum frequency generation (SFG) surface vibrational spectroscopy carried out under reaction conditions at high pressures of ethylene and hydrogen [293; 305]. The main surface species were found to be di- $\sigma$-bonded ethylene, and ethylidyne, which have a strong bond to Pt. However, although the relative concentration of these two species was found to be different on $\operatorname{Pt}(111)$ and $\operatorname{Pt}(100)$, a similar ethylene hydrogenation activity was observed for these two surfaces. Therefore, it could be concluded that these two species are not reaction intermediates, but only stagnant spectators in this reaction [293; 305]. However, at reaction condition where the turnover frequency is high, small amounts of other adsorbate species, namely, $\pi$-bonded ethylene and ethyl were found to be present on the surface. These two species are weakly bounded and are the actual intermediate species for this reaction. Thus, the reaction mechanism could be described as follows: at low temperature and before the onset of the reaction, the Pt surface is covered completely with strongly bonded (di- $\sigma$-bonded) ethylene and ethylidyne. Due to steric considerations, a complete surface coverage of these adsorbates still leaves vacant sites that allow the hydrogen dissociation. At high ethylene pressure, the ethylidyne groups are pushed to the side to open surface sites in which ethylene can adsorb weakly ( $\pi$-bonded) [308] and immediately hydrogenate to ethyl and ethane. Following this mechanism, only a small fraction of the surface (e.g. 0.04 on $\mathrm{Pt}(111)$ [305]) is actively involved in 
the hydrogenation of ethylene. Thus, the origin of the observed structure insensitivity is the poisoning of the majority of surface sites with stagnant strongly bonded species.

In case of methanation $\left(\mathrm{CO}+3 \mathrm{H}_{2} \rightarrow \mathrm{CH}_{4}+\mathrm{H}_{2} \mathrm{O}\right)$ reaction the similar rates observed on on $\mathrm{Ni}(111)$ and $\mathrm{Ni}(100)$ surfaces was found to be due to accumulation of carbonaceous intermediates that have very similar structures on both surfaces [309]. Another example is the synthesis of formate $\left(\mathrm{CO}_{2}+\frac{1}{2} \mathrm{H}_{2} \rightarrow \mathrm{HCOO}_{\mathrm{a}}\right)$ on $\mathrm{Cu} \mathrm{NPs}$, which is insensitive to the type of copper surface selected [e.g. $\mathrm{Cu}(111)$ or $\mathrm{Cu}(110)$ ] [310]. This reaction follows an Eley-Rideal type mechanism, in which gaseous $\mathrm{CO}_{2}$ molecules directly react with adsorbed hydrogen atoms on $\mathrm{Cu}(\mathrm{H}$ coverage 1) [310]. Figure 23 shows the results of $a b$ initio density functional theory (DFT) calculations [300]. There are two different adsorbed formate species on copper surfaces, namely monodentate and bidentate, as shown in Figure 23(a,b). In the monodentate case, one oxygen atom is bound to the copper, while in the bidentate formate, both oxygen atoms are bound to copper atoms. It was found that the initial intermediate product of $\mathrm{CO}_{2}$ with surface atomic hydrogen is the monodentate formate [Figure 23(a)] which has similar adsorption energies on $\mathrm{Cu}(111), \mathrm{Cu}(100)$, and $\mathrm{Cu}(110)$. Although the bidentate formate [Figure 23(b)], which is the final stable configuration, has significantly different binding energy on different $\mathrm{Cu}$ surfaces, the similar transition states that define the net reaction rate [311] lead to similar energetic pathways and a structure insensitivity [300]. The activation energies obtained from Figure 23(d,e) for the synthesis of formate are $0.69 \mathrm{eV}$ on $\mathrm{Cu}(111)$ and $0.64 \mathrm{eV}$ on $\mathrm{Cu}(110)$ are not showing any significant structure dependency. This is in good agreement with the values obtained experimentally $(0.58$ and $0.62 \mathrm{eV}$, respectively) [310]. However, the calculated activation energies for the reverse reaction (the decomposition of bidentate formate) are $0.97 \mathrm{eV}$ on $\mathrm{Cu}(111)$ and $1.44 \mathrm{eV}$ on $\mathrm{Cu}(110)$, also in agreement with the experimental data of $1.12 \mathrm{eV}$ on $\mathrm{Cu}(111)$ and $1.50 \mathrm{eV}$ on $\mathrm{Cu}(110)$, but clearly showing a strong structure dependence [310]. 


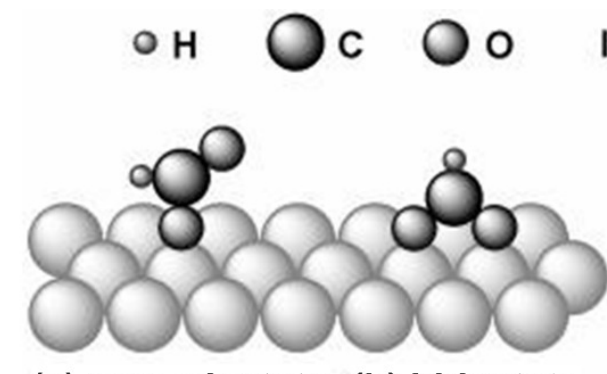

(a) monodentate (b) bidentate

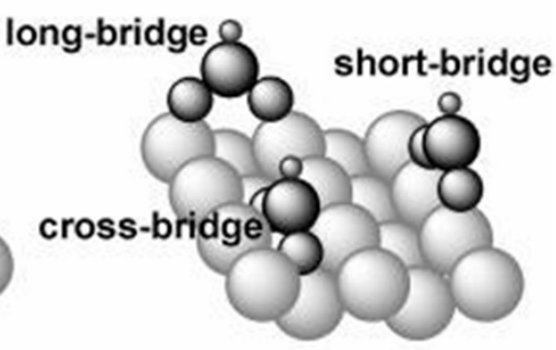

(e) $\mathrm{Cu}(110)$

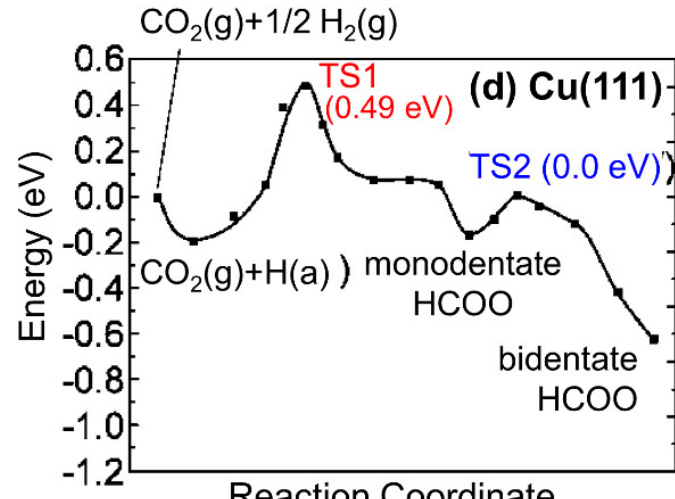

Reaction Coordinate

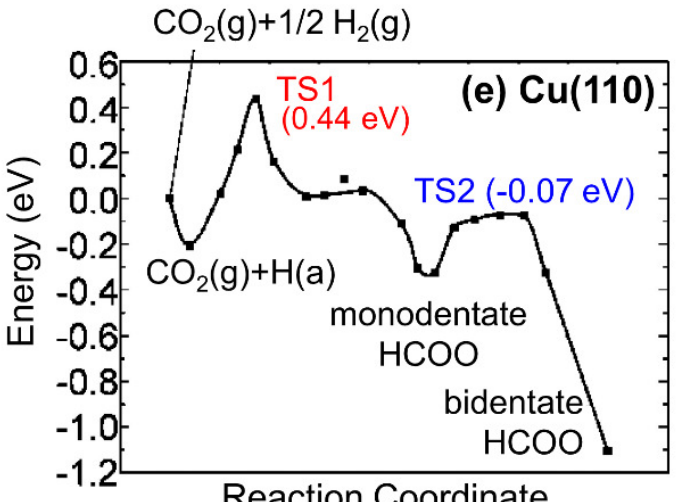

Reaction Coordinate

Figure 23: Side view of the structure of HCOO in (a) monodentate form and (b) bidentate form. (c) Possible adsorption sites of the bidentate $\mathrm{HCOO}$ on $\mathrm{Cu}$ (110). Reaction coordinates for $\mathrm{HCOO}$ synthesis and decomposition on (d) a $\mathrm{Cu}(111)$ surface and (e) a $\mathrm{Cu}(110)$ surface, respectively. Adapted from Ref. [300].

In summary, it could be concluded that in most cases the structure insensitivity reported for a number of chemical processes originates from surface poisoning, an effect which might wash away the distinct surface structures by altering the surface composition. In other cases, the structure insensitivity stems from the fact that the rate determining intermediate species only requires adsorption on a single surface atom and therefore, is not sensitive to the arrangement of neighboring atoms (e.g. Figure 23). Nevertheless, a large fraction of catalytic reactions show moderate to strong structure sensitivity that can be separated into three categories: (i) positive sizeeffects, in which small NPs show higher reaction rates, (ii) negative size-effects with the opposite trend, and (iii) a volcano behavior in which an specific optimum NP size provides the highest activity as compared to smaller or larger NPs.

Examples of reactions with positive size-effects are the decomposition of methanol over $\mathrm{Pt} / \mathrm{TiO}_{2}$ [312], direct propylene epoxidation over $\mathrm{Ag}$ nanoclusters supported on amorphous alumina films[313], and the electrooxidation of formic acid by unsupported Pd NPs [314]. Negative size-effects include oxygen electroreduction reactions on Pt NPs[86], CO oxidation over 
$\mathrm{Ru} / \mathrm{SiO}_{2} / \mathrm{Si}$ [315], and $\mathrm{NO}$ oxidation over $\mathrm{Pt} / \mathrm{Al}_{2} \mathrm{O}_{3}[301 ; 316]$. Other reactions showing the volcano behavior are the water-gas-shift reaction over $\mathrm{Au}$ and $\mathrm{Cu}$ nanoclusters supported on $\mathrm{CeO}_{2}(111)$ and $\mathrm{Au} / \mathrm{ZnO}(000 \overline{1})$ [317], the oxidation of $\mathrm{CO}$ on $\mathrm{Au} / \mathrm{TiO}_{2}(110)[5 ; 318]$ and $\mathrm{Pt} / \mathrm{Al}_{2} \mathrm{O}_{3}$ [316], and the hydrogenation of acetylene over $\mathrm{Au} / \mathrm{Al}_{2} \mathrm{O}_{3}$ [319].

It should be kept in mind that it is possible for a reaction with volcano behavior to be mistakenly considered as to have a positive or negative size-effect. This could happen if the size range studied is not wide enough and falls only into the left or right side of the volcano trend. In addition, the size-dependent activity is also governed by the product of interest. Since the NP size could have a significant effect on the reaction selectivity, it is possible for the size-effect to be positive for one product while negative for another. For instance in the $\mathrm{CO}_{2}$ electroreduction reaction over $\mathrm{Cu} N \mathrm{NP}$, the $\mathrm{H}_{2}$ and $\mathrm{CO}$ production has a positive size-effect, while the methane and ethylene generation shows a negative size-effect [320].

The effect of the NP size on catalytic performance is not limited to the reaction rate, since size-dependent selectivity has also been reported for many reactions [101; 298; 320-323]. Figure 24 shows how the size of $\mathrm{Au}$ NPs supported on anatase $\mathrm{TiO}_{2}$ could affect the reaction selectivity, going from propylene epoxidation on NPs $>2 \mathrm{~nm}$ to propylene hydrogenation on smaller ones.
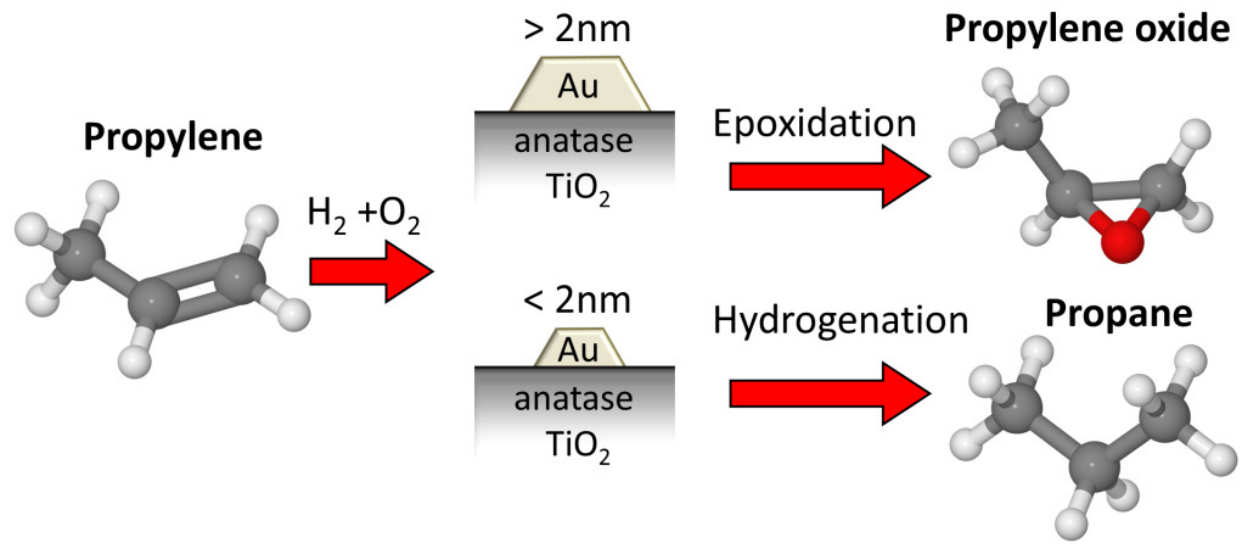

Figure 24: Schematic representation of the size dependence change in the selectivity of the reaction of propylene with oxygen and hydrogen over $\mathrm{Au} / \mathrm{TiO}_{2}$ catalysts. Adapted from Refs. [321; 322].

The effect of the NP size on catalytic activity can be understood based on the binding strength of adsorbates and intermediate species on the NP surface. In a simple reaction in which a diatomic molecule dissociates and reacts with another more readily available molecule, the rate-limiting reaction step is the dissociation of the first molecule [324]. Such step might be strongly influenced 
by the structure of the selected catalyst. For instance, the key step in ammonia synthesis $\left(\mathrm{N}_{2}+3 \mathrm{H}_{2}\right.$ $\left.\rightarrow 2 \mathrm{NH}_{3}\right)$ is $\mathrm{N}_{2}$ dissociation $\left(\mathrm{H}_{2}\right.$ more readily dissociates), and in Fischer-Tropsch synthesis $\left[\mathrm{nCO}+(2 \mathrm{n}+1) \mathrm{H}_{2} \rightarrow \mathrm{C}_{\mathrm{n}} \mathrm{H}_{2 \mathrm{n}+2}+\mathrm{nH}_{2} \mathrm{O}\right]$ is the dissociation of $\mathrm{CO}$ [324]. Likewise, NO reduction is based on NO activation [204], and some oxidation reactions are driven by the rate of $\mathrm{O}_{2}$ dissociation [301]. However, to estimate the reaction rate, two factors should be considered: (i) the dissociation rate of the diatomic molecule, and (ii) the removal rate of products from the surface. The former is determined by the activation (dissociation) energy $\mathrm{E}_{\mathrm{a}}$, and the latter by the binding energy of intermediates, $\Delta \mathrm{E}$. A catalyst would have a high reaction rate if the activation energy is low and the binding of the reaction intermediates is weak. However, in many cases there exists a correlation between the activation energy and binding energies of intermediates $[215 ; 220 ; 324-$ 329]. A Brønsted-Evans-Polanyi relationship shows a linear correlation between the activation energy and the binding energy; a surface that dissociates a molecule readily tends to bind intermediates strongly and vice versa [325; 326]. This behavior results in volcano-type activity, as illustrated in Figure 25(b) $[215 ; 220 ; 324 ; 327 ; 328 ; 330-332]$. Low activation energy helps the dissociation step (high sticking probability), but the concomitant high binding energy would make it hard for the intermediates to leave the surface. In that case, the free surface available for other reactants is minimal, and therefore, the reaction rate would be low, Figure 25 (b,c). When the activation energy is high, the dissociation step will be rate-limiting, and although enough free surface might be available, the low dissociation probability would be detrimental to the reaction rate, Figure 25 (b,c). A similar Brønsted-Evans-Polanyi relationship can also be obtained for a given metal but different adsorbates, with those having lower activation energy displaying higher binding energy [328]. 

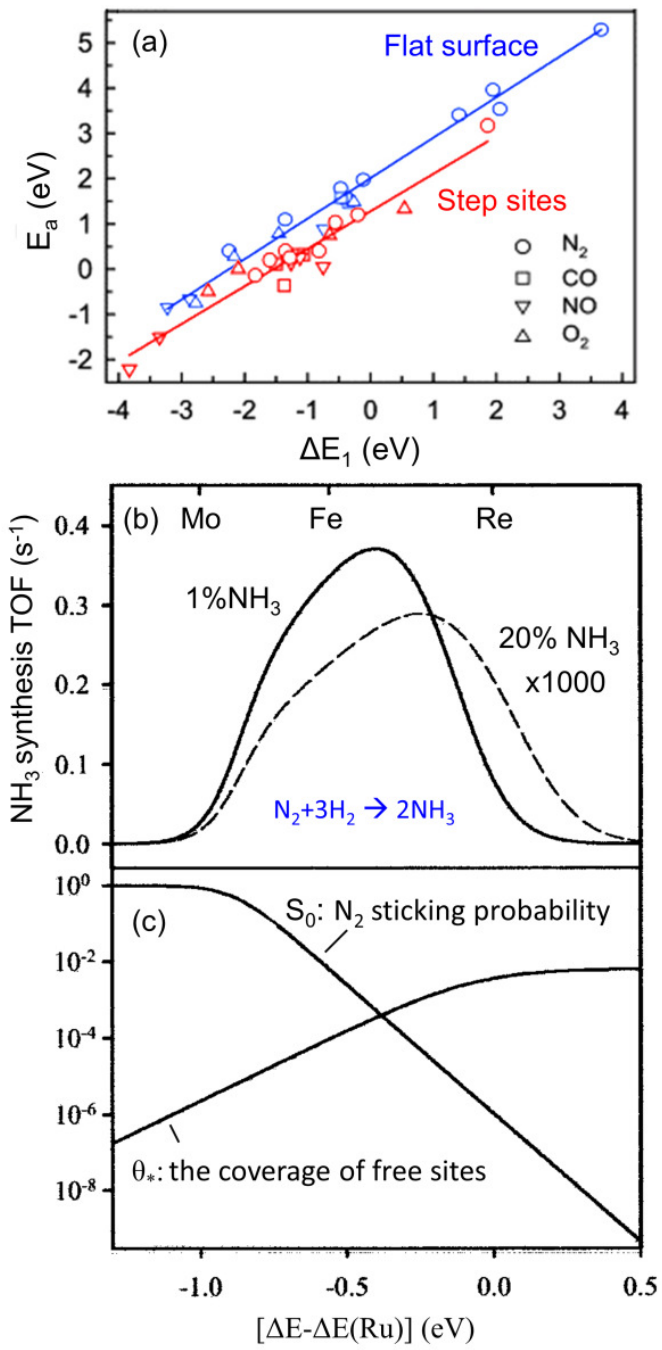

Figure 25: (a) Calculated activation energies $\left(\mathrm{E}_{\mathrm{a}}\right)$ for $\mathrm{N}_{2}, \mathrm{CO}, \mathrm{NO}$, and $\mathrm{O}_{2}$ dissociation on a number of different metals (including $\mathrm{Mo}, \mathrm{Fe}, \mathrm{Ru}, \mathrm{Co}, \mathrm{Rh}, \mathrm{Ir}, \mathrm{Ni}, \mathrm{Pd}, \mathrm{Pt}, \mathrm{Cu}, \mathrm{Ag}, \mathrm{Au}$ ) plotted as a function of the calculated dissociative chemisorption potential energy for the dissociation products $(\Delta \mathrm{E})$. The results are calculated for flat surfaces and step sites. (b) Ammonia synthesis rate (TOF) as a function of the $\mathrm{N}_{2}$ dissociative chemisorption energy of nitrogen $(\Delta \mathrm{E})$ relative to that on ruthenium $\left[\Delta \mathrm{E}_{\mathrm{Ru}}\right] . \Delta \mathrm{E}$ varies continuously and some of the values that match certain metals are marked on the top axis (e.g. Mo, Fe, Re). The rates are calculated by combining the BrønstedEvans-Polanyi relation with the microkinetic model for ammonia synthesis. The TOFs are obtained at $673 \mathrm{~K}$ and 100 bar in a stoichiometric $\mathrm{H}_{2}: \mathrm{N}_{2}$ gas mixture containing $1 \%$ or $20 \%$ (dashed) $\mathrm{NH}_{3}$. (c) The $\mathrm{N}_{2}$ sticking probability, $S_{0}$, and the coverage of free sites, $\theta *$, on the surface for each value of the adsorption energy are provided. These results refer to the case where there is $1 \%$ conversion. It can be seen how the maximum in activity is a compromise between a high sticking probability (low barrier for $\mathrm{N}_{2}$ dissociation) and a large number of free sites (low $\mathrm{N}$ and $\mathrm{NH}$ coverage) where reaction can occur. Adapted from Ref. [329]. 
Another closely related phenomenon is the so called "compensation effect" [333-337]. This effect is described as a linear correlation between the natural log of the pre-exponential factor and the apparent activation energies for a set of different catalysts (different metal, loading, synthesis, pretreatment, etc). In this case, a decrease in the activation energy that should result in an increase of the reaction rate is accompanied by a decrease in the prefactor, that would lead to a decrease of the reaction rate. Therefore, the reaction rate is determined by the interplay between the prefactor and the activation energy, and often results in a volcano type behavior. We will show examples of this phenomenon in section 3.4 for the NO oxidation reaction [301].

Although the compensation effect has been known in the catalysis community for a long time [337], and despite the simplicity of the compensation relation, there is still no universally accepted explanation for this phenomenon [338]. For instance, it has been shown that in some cases, random [339] or systematic [340] experimental errors could result in an apparent compensation effect. These issues has been discussed in the literature for more than 50 years [341] and the underlying problem was pointed out to be the fact that both parameters (pre-exponential factor and the activation energy) are obtained from the same data sets and therefore, are not statistically independent [341]. Although a careful statistical method has been devised to distinguish such artifacts from real effects [336; 342-344], there has been a lot of confusion in the literature due to some studies neglecting this issue [343]. However, this discussion should not cause any mistrust in the compensation effect as a real phenomenon with significant physicochemical implications [345].

It has been argued that the compensation effect is the result of the dynamical nature of the entropy providing more excitation channels for reactions with higher energy barrier, which leads to an increase in the pre-exponential factor [346; 347]. Another explanation that has been put forward is the change in the kinetic regime [334]. In this approach, when high energy barriers are present, the reaction kinetic is dominated by the rate of reactant activation, while when the energy barrier is low, the reaction kinetics is mainly influenced by the stability of the reaction products [334]. Following this approach, the underlying reason for the compensation effect is the BrønstedEvans-Polanyi relationship [334]. Readers interested in gaining further insight into this effect are referred to the following detailed reviews $[338 ; 345 ; 346 ; 348 ; 349]$.

Now that it has been established that tuning the binding energy of adsorbates can be used to turn on or optimize a catalytic reaction, the question to answer is how such binding energy may be 
manipulated. One approach is to exploit the NP size-effect, which will be discussed in more detail in the next section. Another method that has received significant attention by the catalysis community is the use of bimetallic systems in order to tune a variety of material properties, including chemical reactivity [28; 201; 350-355]. Figure 26(a,b) compares TPD results following the hydrogenation of 0.5 langmuir of acrolein on $\mathrm{Pt}-\mathrm{Ni}-\mathrm{Pt}(111)$, Ni-Pt-Pt(111), and $\mathrm{Pt}(111)$ after the coadsorption of acrolein and atomic hydrogen on these surfaces at $100 \mathrm{~K}$. Figure 26(a) displays the TPD spectra of mass $31 \mathrm{amu}$, which is a cracking fragment for both, $\mathrm{CH}_{2}=\mathrm{CHCH}_{2} \mathrm{OH}$ (2propenol) and $\mathrm{CH}_{3} \mathrm{CH}_{2} \mathrm{CH}_{2} \mathrm{OH}$ (1-propanol), and Figure 26(b) shows mass 58, which is a cracking fragment for $\mathrm{CH}_{2}=\mathrm{CHCH}_{2} \mathrm{OH}$ and $\mathrm{CH}_{3} \mathrm{CH}_{2} \mathrm{CH}=\mathrm{O}$ (propanal). The production of either 2-propenol or 1-propanol is not detected on $\operatorname{Pt}(111)$ and $\mathrm{Ni}-\mathrm{Pt}-\operatorname{Pt}(111)$, as indicated by the absence of any desorption peaks in the $31 \mathrm{amu}$ TPD spectra of the two surfaces. In contrast, a relatively intense desorption peak at $260 \mathrm{~K}$ is observed from the $\mathrm{Pt}-\mathrm{Ni}-\mathrm{Pt}(111)$ structure. This peak is assigned to the formation of primarily 2-propenol, based on the peak intensity ratio in the 31 and $60 \mathrm{amu}$ spectra. Figure 26(c) shows DFT calculations from the former three structures that provide insight into the origin of the distinct selectivity observed for acrolein hydrogenation. Three binding configurations of acrolein on differente surfaces are considered: (i) di- $\sigma-\mathrm{C}-\mathrm{C}$, (ii) di- $\sigma-\mathrm{C}-\mathrm{O}$, and (iii) $\eta_{4}(\mathrm{C}, \mathrm{C}, \mathrm{C}, \mathrm{O})$.. The DFT results show that the binding energy values of acrolein on the different surfaces are ranked as follows: $\mathrm{H} / \mathrm{Pt}-\mathrm{Ni}-\mathrm{Pt}(111)<\mathrm{H} / \mathrm{Pt}(111)<\mathrm{H} / \mathrm{Ni}-\mathrm{Pt}-\mathrm{Pt}(111)$, similar to those of atomic hydrogen. Therefore, it appears that the higher hydrogenation activity on $\mathrm{H} / \mathrm{Pt}-\mathrm{Ni}-\mathrm{Pt}(111)$ is related to the weaker bonding of both acrolein and atomic hydrogen. The selective hydrogenation pathway on $\mathrm{H} / \mathrm{Pt}-\mathrm{Ni}-\mathrm{Pt}(111)$ is most likely due to the similar binding energy values of the three adsorption configurations of acrolein, which would lead to its adsorption through both the $\mathrm{C}=\mathrm{O}$ and $\mathrm{C}=\mathrm{C}$ moieties, making it possible to hydrogenate both bonds. On the other hand, as predicted by DFT, the adsorption of acrolein on $\mathrm{H} / \mathrm{Pt}(111)$ and $\mathrm{H} / \mathrm{Ni}-\mathrm{Pt}-\mathrm{Pt}(111)$ surfaces does not occur through the di- $\sigma-\mathrm{C}-\mathrm{O}$ configuration, hence making it difficult to hydrogenate the $\mathrm{C}=\mathrm{O}$ bond, as confirmed by the absence of the 2-propenol product in TPD measurements. 

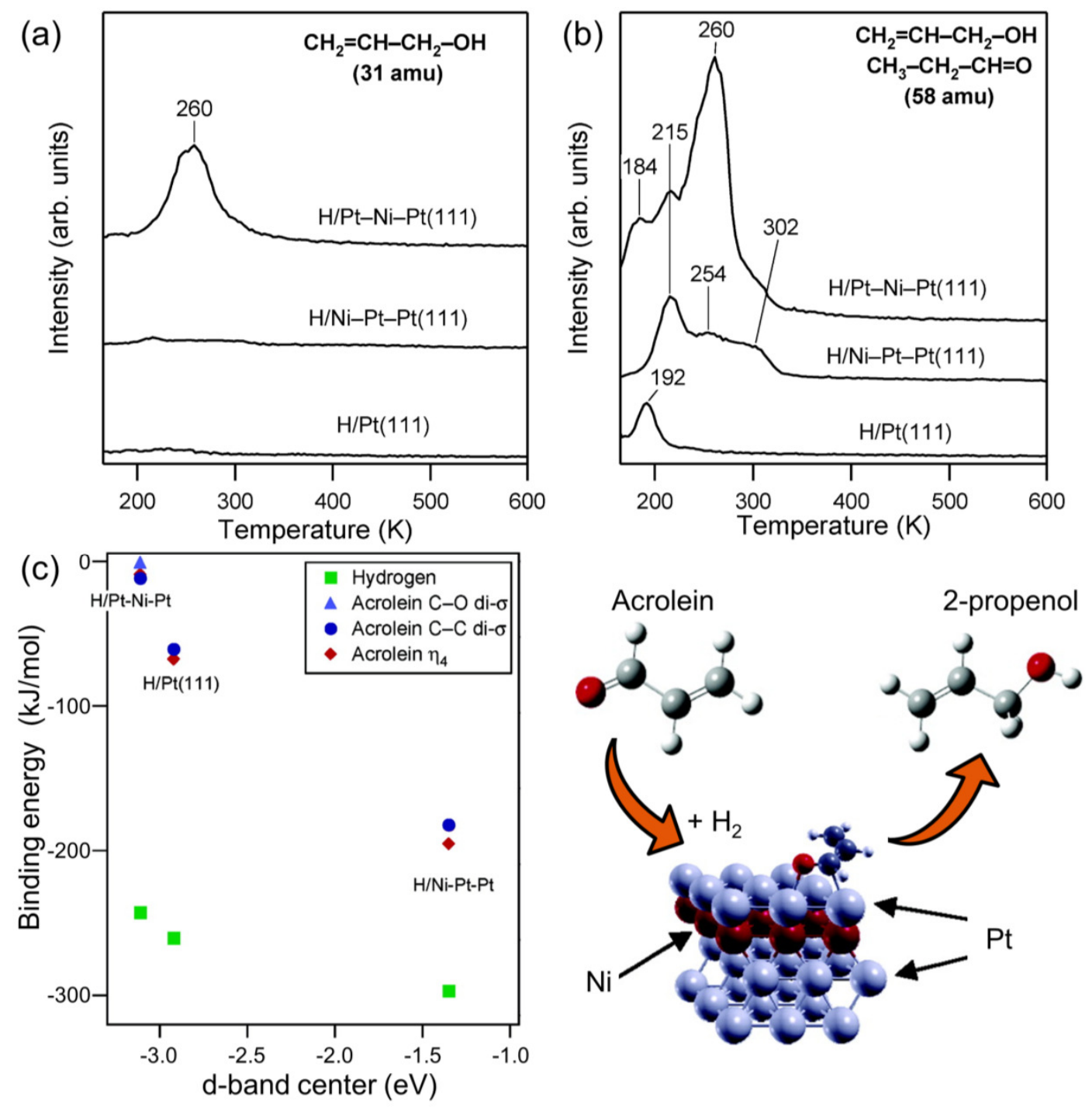

Figure 26: TPD spectra following the reaction of acrolein with coadsorbed atomic hydrogen at $100 \mathrm{~K}$ on different surfaces: (a) 2-propenol (31 amu), (b) propanal and 2-propenol (58 amu). (c) DFT calculations of acrolein and hydrogen binding energies vs the surface d-band center for $\mathrm{H} / \mathrm{Pt}-\mathrm{Ni}-\mathrm{Pt}(111), \mathrm{H} / \mathrm{Pt}(111)$, and $\mathrm{H} / \mathrm{Ni}-\mathrm{Pt}-\mathrm{Pt}(111)$ surfaces. (d) Schematic of acrolein conversion to 2-propenol on $\mathrm{Pt}-\mathrm{Ni}-\mathrm{Pt}(111)$ surface. Adapted from Ref. [350].

One of the most exciting examples of the enhanced activity of NPs is given by Au NPs. Although bulk gold is widely known to be catalytically inert [356], Au NPs smaller than $\sim 5 \mathrm{~nm}$ were found to be excellent catalysts for $\mathrm{CO}$ oxidation reactions $[6 ; 71 ; 357-361]$, selective epoxidation of propylene [321], water-gas shift reactions [362], NO reduction [363], oxidation of alcohols [364], and other chemical processes [4; 365-367]. Some of these reactions will be discussed in more detail in the next section. 
To explain the enhanced activity of small NPs, different models have been proposed [269; 368-371] following a variety of phenomena involved in the peculiar behavior of nanoscale materials. A graphical summary of some of these phenomena is provided in Figure 27. Some of these phenomena include: (i) small metal clusters displaying non-metallic behavior (quantum sizeeffects), (ii) the presence of higher densities of low-coordinated atoms at corners and edges, (iii) NP-support charge transfer, (iv) NP-support interactions (active perimeter sites), (v) sizedependent NP oxidation and stabilization of surface oxides. In the next sections we discuss each of these phenomena in more detail.

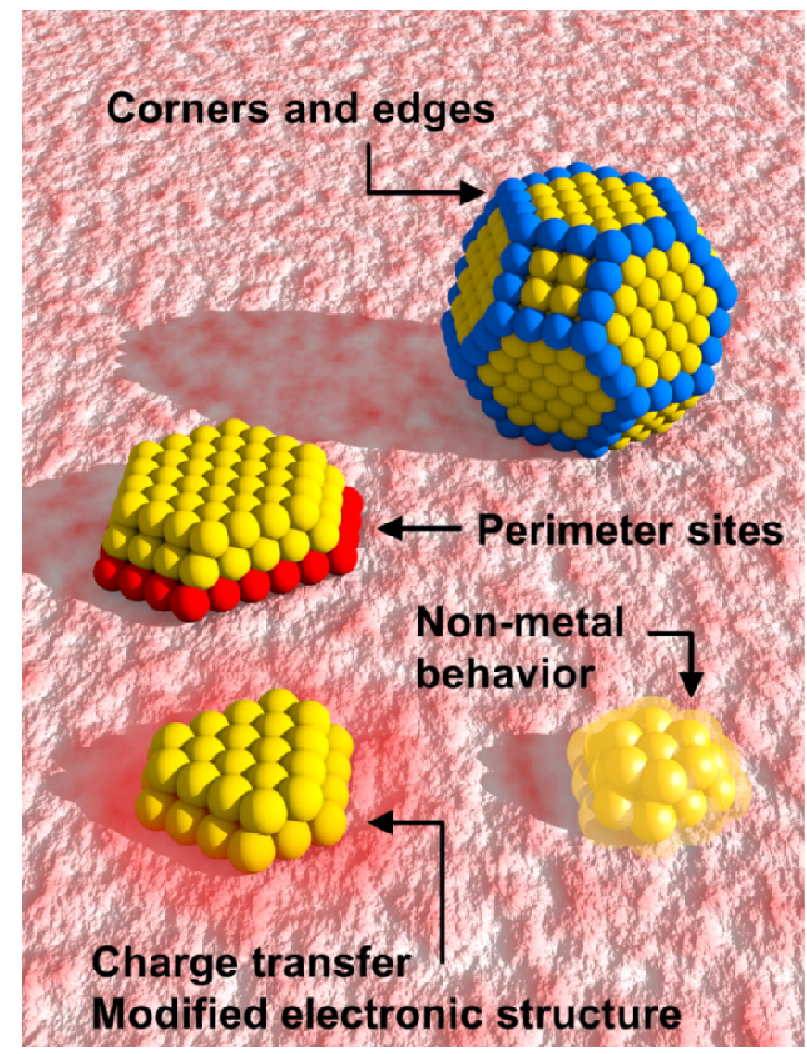

Figure 27: Different phenomena involved in the unusual reactivity of supported metal particle catalysts.

It should be however noted that the unusual physical/chemical properties of small NPs may not always be beneficial for a particular application. Specifically, in some cases it has been shown that NPs with large size have a better performance or selectivity than smaller NPs $[315 ; 316 ; 323$; 372]. For instance, in the ethylene epoxidation reaction, the selectivity toward ethylene oxide of large Ag NPs $(\sim 1 \mu \mathrm{m})$ was found to be about $45 \%$, while that of NPs $<30 \mathrm{~nm}$ was only $25 \%$ [323]. Larger Pt NPs were also found to be more active for the combustion of o-xylene and m-xylene 
[249]. In NO oxidation reaction over Pt NPs, smaller NPs were found to be less reactive than larger NPs [301]. Using in situ and in operando x-ray absorption spectroscopy, the origin of this observation was found to be the fact that smaller NPs are more prone to oxidation, which was found to be detrimental for this particular catalytic process [301; 316;372]. On the other hand, for chemical reactions where oxidized metal surfaces might be more active (i.e. partial oxidation reactions), smaller NPs could display superior catalytic performance [16; 73; 373].

The sections below describe some of the effects reported in the literature responsible for the distinct reactivity or chemisorption behavior of NPs.

\subsection{Quantum size-effects}

The electronic structure of nanosized metal particles differs from that of their bulk counterparts. This is due to the confinement of conduction electron waves inside the NPs as well as the alteration of their band structure due to finite size effects $[5 ; 113 ; 210 ; 239 ; 374-382]$.

For small metallic NPs, the continuous band structure expected for an infinite solid crystal breaks down to discrete levels: the smaller the NP, the larger will be the Kubo gap [383], which is the average spacing between consecutive electronic levels. Figure 28 illustrates the formation of discrete energy levels and the eventual metal to insulator transition with decreasing NP size [384]. In the early sixties, Kubo was among the first researchers to suggest that such alteration of the electronic structure of the NPs could have profound effects on other NP physical/chemical properties $[383 ; 385]$. Adding to the complexity, it has been reported that such alteration in the NP electronic structure is non-monotonic, and that NPs with a certain number of atoms, known as magic numbers, could form closed-shell structures with a significantly different electronic structure as compared to their immediate neighbors with just a few atoms more or less [380; 386]. 


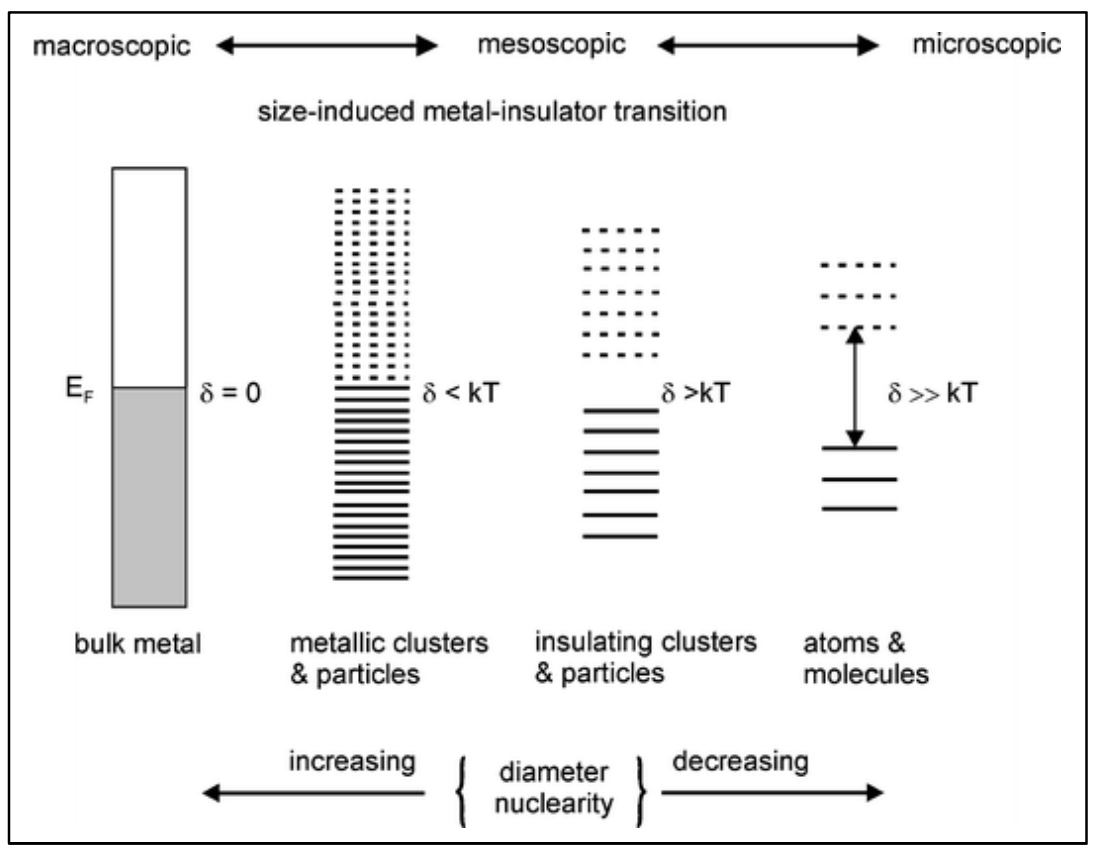

Figure 28: Evolution of the electronic structure and the density of states with increasing NP size (from right to left) for metallic systems [384]. $\delta$ is the Kubo gap.

Goodman's group was among the first to suggest that the onset of the high activity of small gold particles might be related to a metal to non-metal transition for NP sizes where quantumsizeeffects set in [5; 387]. By measuring the band gap of Au NPs deposited on $\mathrm{TiO}_{2}(110)$ using scanning tunneling spectroscopy (STS), a size-dependence was observed, with clusters with less than three atomic layers showing a non-metallic behavior (band gap of 0.2-0.6V), while thicker NPs were essentially metallic, Figure 29(b) [5]. The CO oxidation activity of Au NPs was also shown to undergo a transition within the same size range from almost nothing to high conversion [Figure 29(c)], which suggested that quantum size-effects might underlie the high activity observed [5]. 

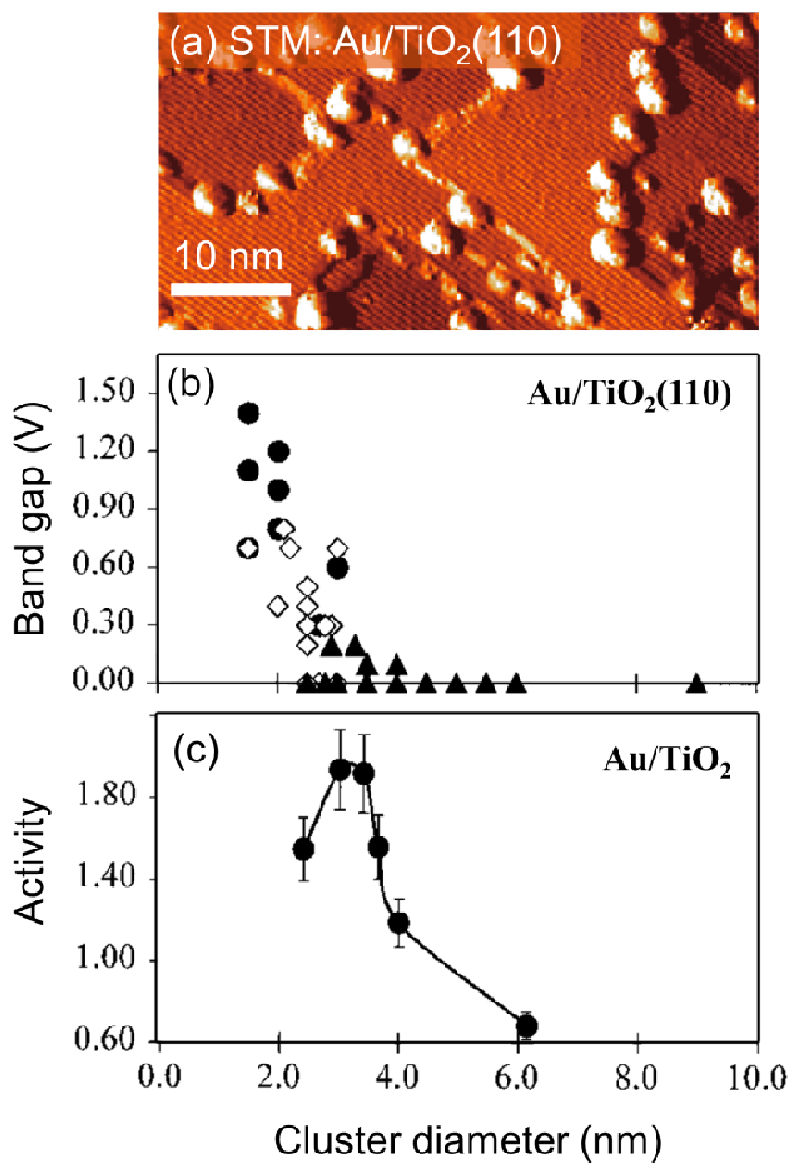

Figure 29: (a) STM image of $\mathrm{Au} \mathrm{NPs} / \mathrm{TiO}_{2}(110)-(1 \times 1)$ as prepared before a $\mathrm{CO}$ oxidation reaction. The Au coverage is $0.25 \mathrm{ML}$, and the sample was annealed at $850 \mathrm{~K}$ for $2 \mathrm{~min}$ [5]. (b) Cluster band gap measured by STS as a function of the Au cluster size supported on $\mathrm{TiO}_{2}(110)$ $(1 \times 1)$. The band gaps were obtained while the corresponding topographic scan was acquired on various $\mathrm{Au}$ coverages ranging from 0.2 to $4.0 \mathrm{ML}$. (•) Two-dimensional (2D) clusters; ( $\square$ ) 3D clusters, two atomic layers in height; $(\boldsymbol{\Delta})$ 3D clusters with three atomic layers or greater in height. (c) The activity for $\mathrm{CO}$ oxidation at $350 \mathrm{~K}$ as a function of the Au cluster size supported on $\mathrm{TiO}_{2}(110)-(1 \times 1)$ assuming total dispersion of the $\mathrm{Au}$ NPs. The CO:O $\mathrm{O}_{2}$ mixture was 1:5 at a total pressure of 40 Torr. Activity is expressed as (product molecules) $\times(\text { total Au atoms) })^{-1} \times \mathrm{s}^{-1}$. Adapted from Ref. [5].

Figure 30 shows the results obtained in a follow-up study on small Au NPs supported on a semimetal, TiC, where the band gap was found to initially increase for clusters smaller than $2 \mathrm{~nm}$ in height, but it dropped again for cluster heights below 0.5 nm [388]. In fact, for NPs below 1.3 $\mathrm{nm}$, the contribution of states from the semi-metallic TiC substrate seems to affect the electronic structure of $\mathrm{Au}$ (band gap $\sim 0.8 \mathrm{eV}$ ). Thus, for very small supported NPs, a combination of quantum size-effects and NP-support interactions should be taken into account, an effect that 
should even more strongly affect the previous data by Goodman's group on the larger bandgap $(3.2 \mathrm{eV})$ semiconducting $\mathrm{TiO}_{2}$ support [388].
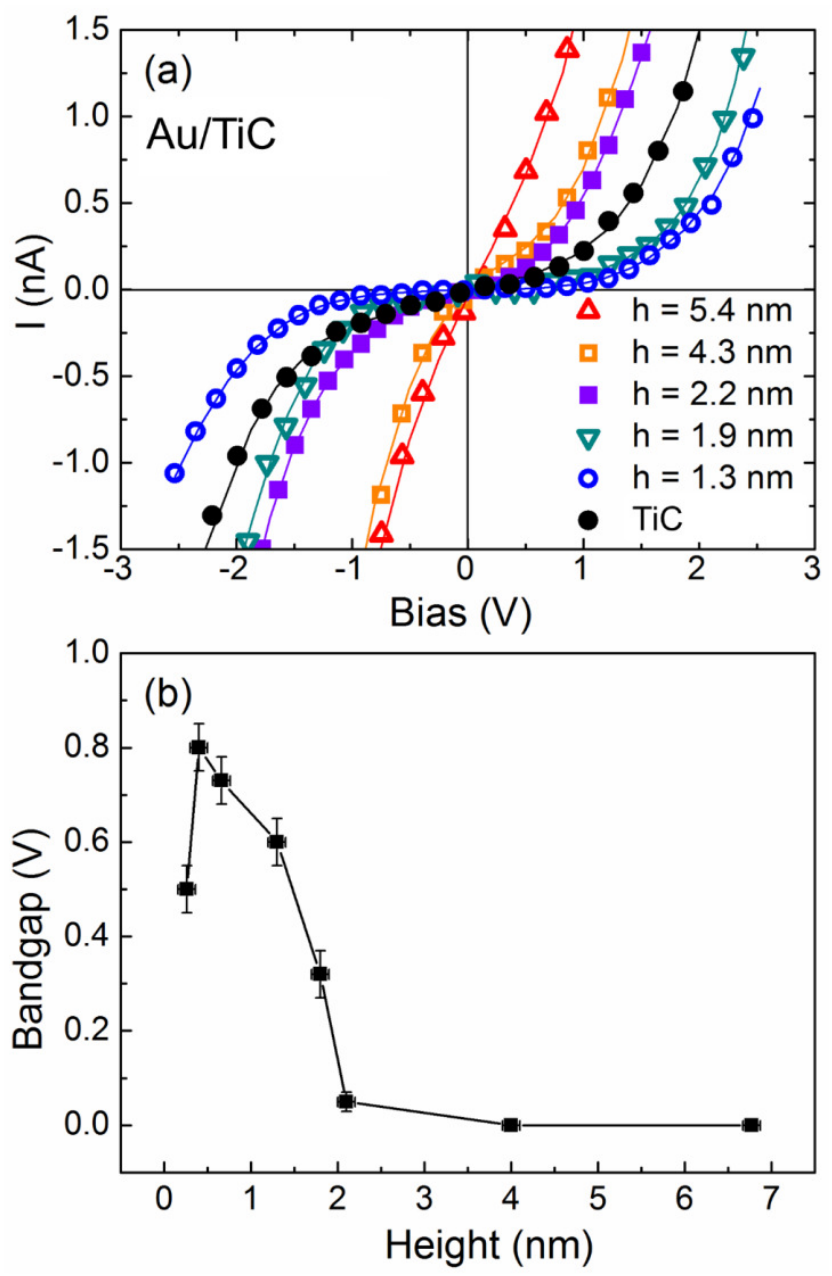

Figure 30: (a) STS measurements on Au NPs with different size supported on thin TiC films showing metallic behavior for large NPs $(5.4 \mathrm{~nm})$ and a bandgap comparable to that of support on smaller NPs $(1.3 \mathrm{mn})$. (b) Summary of band gap values as a function of the NP height. Adapted from Ref. [388].

To investigate the quantum size-effect on the binding energy of $\mathrm{CO}$ and $\mathrm{O}, \mathrm{DFT}$ calculations were carried out on freestanding Pt and Au clusters ranging from 13 to 1415 atoms (0.7-3.5 nm in diameter) [382; 389]. To exclude other factors such as the coordination number of surface atoms, the adsorbates were placed on the cluster in the same local geometries as preferred on platinum (111) and (211) surface slabs. The adsorption energies were all calculated in the low coverage limit, with one single adsorbate per cluster, 1/16 ML coverage relative to the (111) surface on the close-packed slab, and 1/4 ML coverage relative to the step-edge atoms on the (211) surface slab. 
Figure 31 summarizes the adsorbate binding energies obtained for $\mathrm{Au}$ and Pt NPs. For platinum, the surface catalytic properties of the clusters were found to converge to the single crystal limit for clusters with as few as 147 atoms $(1.6 \mathrm{~nm})$. Similar convergence was found to occur in the case of $\mathrm{Au}$ but at a larger NP size, namely, above 560 atoms $(2.7 \mathrm{~nm})$ [382; 389]. This is in agreement with the data shown in Figure 30 in terms of the size range in which the electronic properties of Au NPs converge to those of Au bulk.

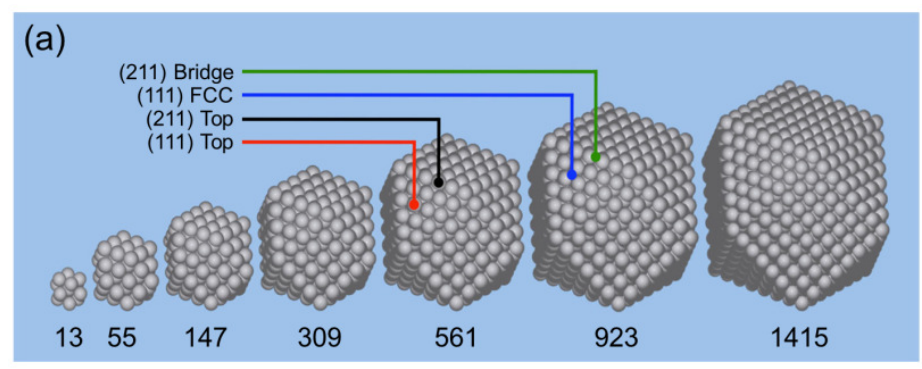

Cluster size Cluster size

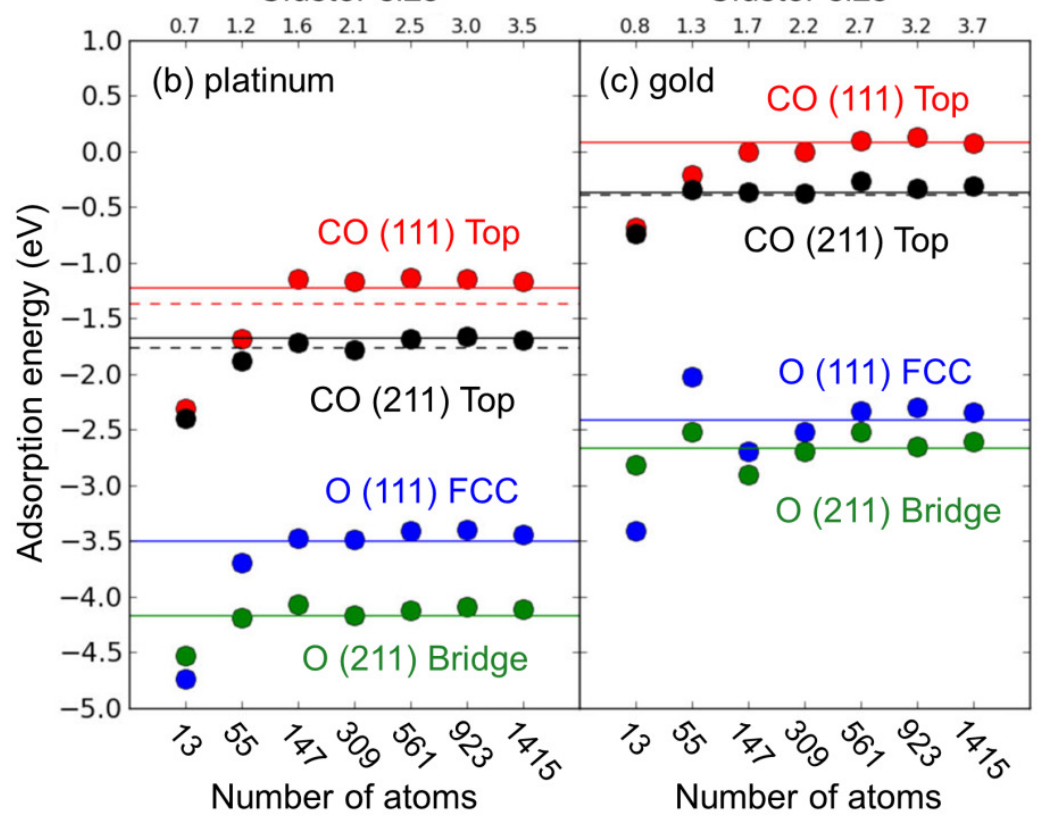

Figure 31: (a) Cuboctahedral platinum clusters used in DFT calculations with two adsorption sites for $\mathrm{CO}$ and $\mathrm{O}$ on every cluster. $\mathrm{CO}$ is adsorbed on top on the two facets and $\mathrm{O}$ is adsorbed 3-fold fcc and bridge on the (111) and (211) facets, respectively. (b),(c) Calculated adsorption energies of $\mathrm{O}$ and $\mathrm{CO}$ on the (111) plane and stepped facet of platinum and gold clusters. (b) Results for platinum where $\mathrm{CO}$ is adsorbed on top on the two facets and $\mathrm{O}$ is adsorbed 3 -fold fcc and bridge on the (111) and stepped facets, respectively. (c) Similar results for adsorption sites identical to the ones on platinum for gold NPs. The horizontal lines indicate the adsorption energies for $\mathrm{O}$ and $\mathrm{CO}$ on the (111) and (211) surface slabs. Experimentally obtained desorption energies for $\mathrm{CO}$ on $\mathrm{Pt}(111), \operatorname{Pt}(211)$, and $\mathrm{Au}(211)$ are shown as dashed lines [390-392]. Adapted from Ref. [382]. 
Although many reports describe the presence of nonmetallic bandgaps in small metal NPs, the effect of such band gaps on catalytic activity is not certain. For instance, DFT calculations by Mills et al. [376] demonstrated that the presence of a band gap does not guarantee a turn on of the catalytic activity. For small $\mathrm{Au}$ NPs $\left(\mathrm{Au}_{1}-\mathrm{Au}_{7}\right)$ it was shown that despite the presence of a bandgap, $\mathrm{O}_{2}$ does not bind to the flat side of the NPs. Additionally, when the same NPs are supported on $\mathrm{Au}(111)$, the bandgap is not observed, but they were found to bind $\mathrm{O}_{2}$ similarly to free clusters, only with a slightly smaller binding energy. Such binding for both free and Au(111)supported NPs occurs only at corners and edges, corroborating that the main criterion to be considered for the understanding of the binding properties of Au NPs is the presence of lowcoordinated atoms, and not the band gap.

TPD studies of $\mathrm{CO}$ adsorption on $\mathrm{Au}$ NPs supported on $\mathrm{Al}_{2} \mathrm{O}_{3}, \mathrm{FeO}(111)$, and $\mathrm{Fe}_{3} \mathrm{O}_{4}(111)$, showed a higher binding energy for the smallest NPs, irrespective of the support [12; 248]. However, using TPD, STM, and infrared reflection absorption spectroscopy (IRAS), no evidence was found for the quantum size-effect in the CO adsorption behavior of Au NPs supported on $\mathrm{FeO}(111)$ thin films [248].

Other phenomena have been observed experimentally resulting from quantum size-effects. For instance, using X-ray photoelectron spectroscopy (XPS), positive binding energy shifts have been observed with decreasing particle size for small metallic clusters [72]. Several models were proposed to explain the origin of such shifts based on the electronic structure of the clusters (initial state effects) [393; 394], as well as on positive charge left on the cluster surface during the photoemission process (final state effects) [71; 395]. In some cases, the NP-support interaction might also play a role on the BE shifts observed on small metal clusters. DFT calculations by Yang et al. [396] suggested that the positive core-level shifts measured on Au NPs supported on $\mathrm{MgO}(001)$ and $\mathrm{TiO}_{2}(110)$ could be related to the presence of oxygen vacancies in the support, and not the final state contribution. For Au NPs deposited on diamond, where a much weaker $\mathrm{NP} /$ support interaction is expected, the size-effect was observed within a smaller size range $(<1.6$ nm) [397]. Based on X-ray-induced photoelectron spectra of the valence-band region, a transition from bulk-like to atomic- or molecular-like properties was observed for mass-selected $\mathrm{Au}_{33}$ clusters, but not for larger NPs $(\geq 1.6 \mathrm{~nm})$ [397]. Therefore, the trends reported for $\mathrm{Au} / \mathrm{TiO}_{2}$ [5], occurring at larger NP sizes [5] cannot be exclusively assigned to quantum size-effects, but must be at least in a significant fraction assigned to the support effect [397]. 
Several theoretical studies have looked into finite size effect phenomena on NPs structure and electronic properties and have linked quantum size-effects to the modified adsorption or catalytic properties detected [382; 398]. However, similar clear direct correlations are more rarely found in the experimental literature. For instance, although several studies have discussed a correlation between size-dependent electronic properties and catalytic activity, it has not been proven that such correlation implies causation. For instance, as it was discussed earlier, a non-metallic behavior (e.g. presence of a bandgap) has been shown for small catalytically active Au NPs supported on $\mathrm{TiO}_{2}[5 ; 387]$. However, later studies have demonstrated that the presence of the bandgap is neither sufficient nor necessary for the enhanced activity [248; 376]. Nevertheless, later theoretical studies also revealed a connection between the HOMO-LUMO gap and the O adsorption energy on Au NPs which in turn could affect their catalytic properties [398].

In another study, a direct link was made between the binding energies of core electrons obtained by XPS and the catalytic activity of size-selected $\operatorname{Pd}_{n}$ NPs ( $n=1$ to 25) [399]. However, quantum size-effects may not be the only explanation for this behavior, but the role of support should be also taken into account $[400 ; 401]$. Such correlation could be due to the fact that both, the changes in the electronic structure, as well as the catalytic activity are induced by size- and structure-dependent charge transfer phenomena from the $\mathrm{TiO}_{2}$ support. Also, non-monotonic catalytic properties have been shown to occur for very small NPs (e.g. those featuring less than 150 atoms), where adding or removing one atom may strongly affect the electronic structure, and consequently the catalytic activity and/or selectivity [265; 401-406]. However, since in this size range the addition or removal of individual atoms could also drastically change the most favorable NP morphology, and while also keeping in mind the support effect, the quantum size-effect cannot be generally singled out experimentally as the sole responsible of the non-monotonic reactivity behavior detected.

In summary, it is well accepted that quantum size-effects occur for small metal NPs, and that they lead to modifications of their electronic properties. Therefore, as has been shown theoretically, it is very likely that such effects may change the catalytic properties of the NPs. However, in experimental setups, the extent of the influence of such effects on catalytic activity is yet to be unveiled, since some of the observed effects might be at least partially induced by other contributions such as NP/support interactions or simple morphological/structural variations in the NPs. 


\subsection{Undercoordinated atoms at the NP surface}

The atomic coordination number $(\mathrm{CN})$ refers to the number of first nearest neighboring atoms. The bulk coordination number of a metal with a FCC structure is 12 , for BCC is 8 , and for a simple cubic structure is 6 . However, the coordination numbers of atoms at extended or NP surfaces could be much smaller. For instance, for FCC metals such as Pt or Au, the coordination numbers of atoms on flat (111) and (100) surfaces are 9 and 8 respectively. This value could drop to 4-7 at step edges of the mentioned surfaces or at corner and edge sites on NPs.

The effect of surface roughness on catalytic performance is widely accepted as a result of the higher content of low-coordinated atoms [255; 407-411]. This can be achieved on extended metal surfaces by scratching [407], ion bombardment [412], or etching [413]. Single crystal surfaces with high Miller indexes also provide a high density of step edges [269; 414; 415]. In the case of NPs, reducing the size usually results in an increase in the population of low-coordinated atoms, Figure 32. This is particularly true for faceted NPs in which the facet area drops with size by a factor of $1 / \mathrm{r}^{2}$, while the number of atoms at edges drops by a factor of $1 / \mathrm{r}$. For spherical NPs, a significant increase in the number of low-coordinated atoms does not occur until the NPs become smaller than a certain size of about 2-3 nm [320].

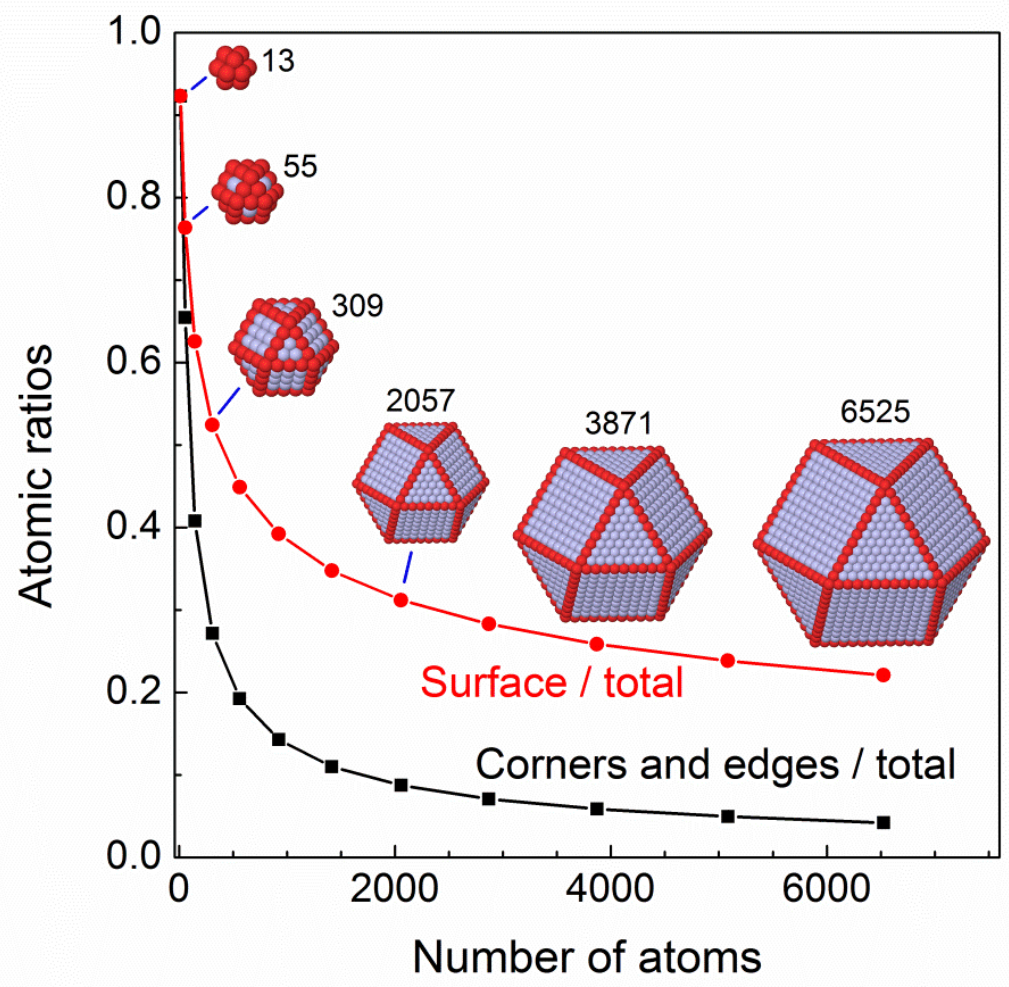


Figure 32: The ratios of (i) the surface atoms to the total number of atoms (red circles) and (ii) atoms at corner and edge sites to the total number of atoms (black squares) obtained for fcc NPs with cuboctahedron shape. Other faceted shapes (e.g. octahedron, truncated cuboctahedron, etc.) would show a similar size dependence.

\section{(a) Adsorption properties}

Low-coordinated atoms usually have an electronic structure that extends outside of the metal and into the vacuum, which makes them available to contribute to adsorbate binding [376]. Enhanced binding of $\mathrm{CO}$ and oxygen to stepped $\mathrm{Au}(211)$ [269] and $\mathrm{CO}$ to $\mathrm{Au}(332)$ [414; 416] surfaces has been observed as compared to smooth $\mathrm{Au}(111)$ surfaces, suggesting that the enhanced activity of small Au NPs is mainly due to the high fraction of low-coordinated atoms present. Figure 33(a) shows the compilation of CO TPD experiments on different Au surfaces with the desorption peaks associated with atomic sites with lower coordination appearing at higher temperatures [390]. Figure 33(b) shows a summary of $\mathrm{CO}, \mathrm{O}$ and $\mathrm{O}_{2}$ binding energies obtained from TPD as a function of the atomic CN values of the adsorption sites with similar trend for all three adsorbates: the lower the coordination number, the higher the binding energy. 

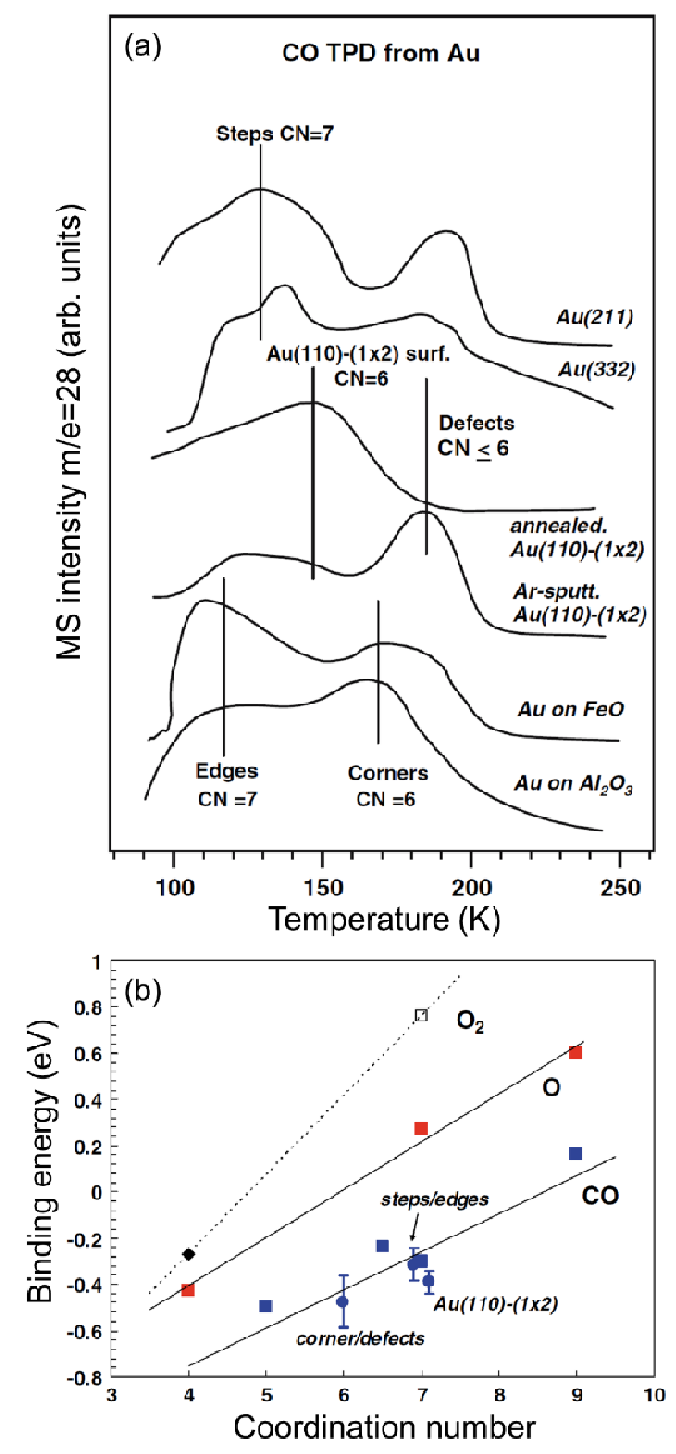

Figure 33: (a) TPD data of $\mathrm{CO}$ on $\mathrm{Au}(211)$ [417], $\mathrm{Au}(332)$ [416], sputtered and annealed $\mathrm{Au}(110)-(1 \times 2)$ [412], and $\mathrm{Au} \mathrm{NPs}$ on flat $\mathrm{Al}_{2} \mathrm{O}_{3}$ and $\mathrm{FeO}$ surfaces [12]. Each trace has been plotted in an arbitrary scale, and does not reflect the absolute amount of $\mathrm{CO}$ desorbing from the different systems. Only relative intensities within a given curve can be compared. (b) Correlation between the binding energies for $\mathrm{CO}, \mathrm{O}_{2}$ and $\mathrm{O}$ atoms on $\mathrm{Au}$ and the coordination number of the $\mathrm{Au}$ atoms in a series of environments. The binding energies $(\mathrm{eV})$ are referred to gas phase $\mathrm{CO}$ and $\mathrm{O}_{2}$. The values for atomic oxygen adsorption are given per $\mathrm{O}$ atom. Since $\mathrm{O}_{2}$ adsorbs on $\mathrm{Au}(211)$ in a metastable state, this value is only tentative. The solid blue dots indicate experimentally determined values for the $\mathrm{CO}$ adsorption energy on steps, edges, and the $\mathrm{Au}(110)-(1 \times 2)$ surface. These values are extracted from CO TPD data on the $\mathrm{Au}(211), \mathrm{Au}(332)$, and $\mathrm{Au}(110)-(1 \times 2)$ single crystal surfaces and from $\mathrm{Au}$ NPs supported on flat $\mathrm{Al}_{2} \mathrm{O}_{3}$ or $\mathrm{FeO}[12 ; 412 ; 416 ; 417]$, assuming a pre-exponential factor of $10^{13}$. The error bars indicate the energy range obtained with a pre-exponential factor between $10^{11}$ and $10^{15}$. 
Figure 34 shows high resolution XPS data of an $\mathrm{Au}(310)$ surface before and after exposure to $\mathrm{CO}$ [418]. The deconvoluted $\mathrm{Au}-4 \mathrm{f}_{7 / 2}$ spectra show three components with slightly different binding energies: (i) bulk, (ii) terrace, and (iii) step sites. The data obtained on the CO-saturated surface at $100 \mathrm{~K}$ and that of the surface under $5 \times 10^{-6}$ mbar CO display similar intensity for the bulk and terrace components after taking into account the bulk signal attenuation by the $\mathrm{CO}$ overlayer. However, the component assigned to step sites shifts significantly to lower binding energies, demonstrating that the adsorption of CO occurs exclusively on step sites [418].
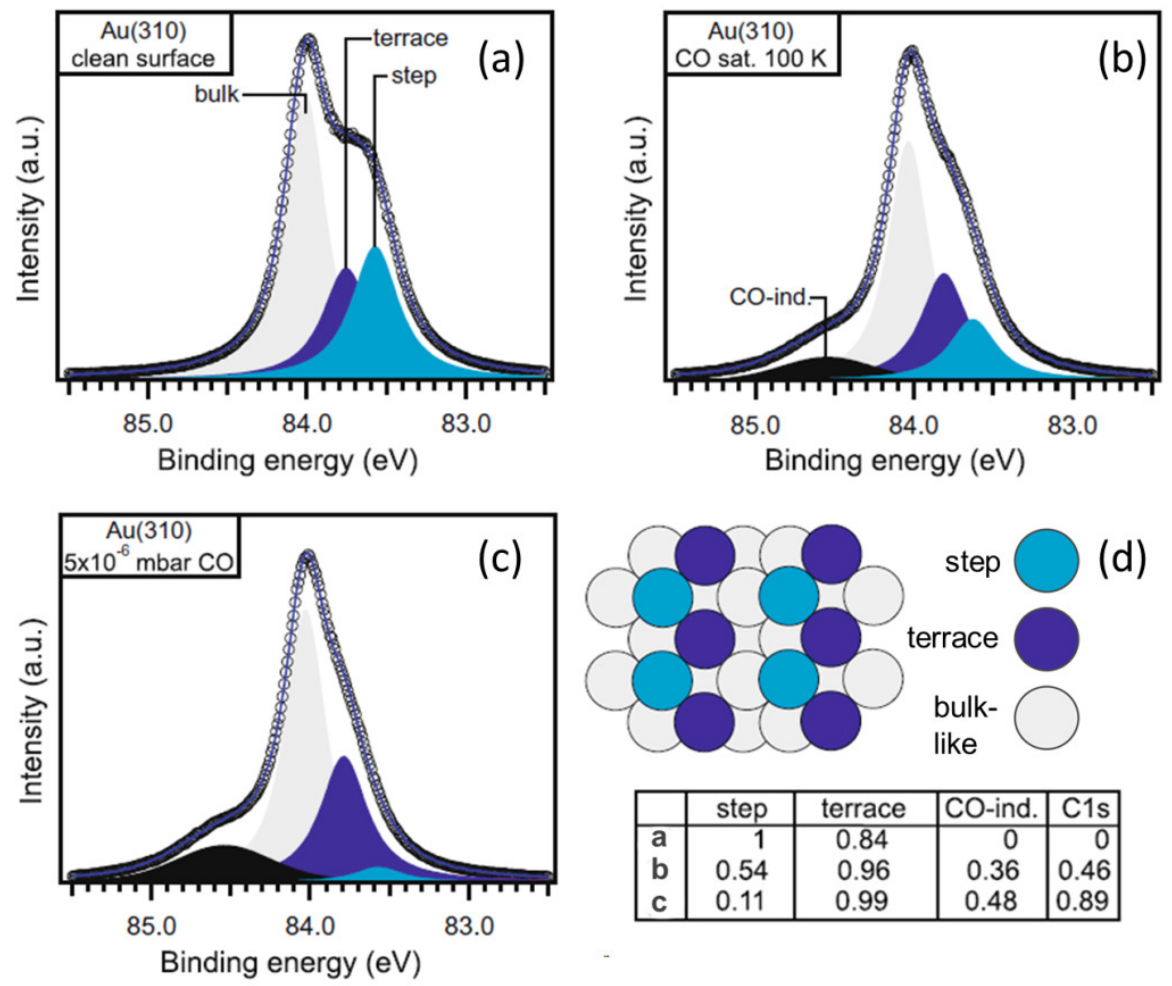

Figure 34: (a-c) High resolution Au- $4 \mathrm{f}_{7 / 2}$ XPS spectra for clean and CO-covered $\mathrm{Au}(310)$ for different $\mathrm{CO}$ coverages. The spectra were deconvoluted into components related to terrace, step and a $\mathrm{CO}$-induced feature. The table shows the relative intensities of the $\mathrm{Au}-4 \mathrm{f}_{7 / 2}$ components obtained from the fit. The corresponding $\mathrm{C}-1 \mathrm{~s}$ intensities, measured directly after acquiring the $\mathrm{Au}-4 \mathrm{f}_{7 / 2}$ spectra, are included as well. (d) Atomic model of the (310) surface with 3 distinctive sites: step (s), terrace (t), and bulk-like sites [418].

Using TPD, STM, and IRAS, the CO adsorption behavior of Au NPs supported on FeO(111) thin films was found to mainly arise from the presence of highly undercoordinated atoms at corner and edge sites [248]. DFT calculations by Mills et al. [376] also demonstrated that the presence of highly undercoordinated atoms on extended surfaces or NPs is the essential requirement for the 
dissociation of $\mathrm{O}_{2}$. However, not all low coordinated atoms perform equally. Atoms with lower $\mathrm{CN}$, or in other words with a higher number of missing bonds, tend to bind adsorbates stronger. This is the main reason for the different activity and selectivity reported for a variety of reactions on (111), (110) and (100) surfaces [177; 419-422].

The thermodynamic diagrams in Figure 35(a-c) show the evolution of the optimum $\mathrm{H}$ coverage on an alumina-supported $\mathrm{Pt}_{13}$ cluster as well as on $\operatorname{Pt}(111)$ and $\operatorname{Pt}(100)$ [19]. These diagrams are obtained by combining $0 \mathrm{~K}$ DFT energies with thermodynamic models. The $\mathrm{H}$ coverage for $\mathrm{Pt}_{13}$ clusters is calculated for the most stable cluster structure. At low hydrogen pressure and high temperature, the hydrogen coverage is zero on all structures, and it increases with increasing pressure and decreasing temperature [19]. For the $\mathrm{Pt}(111)$ surface (coordination number=9), the highest hydrogen coverage is 1 monolayer (ML), while a coverage of $1.75 \mathrm{ML}$ is obtained on $\mathrm{Pt}(100)$ (coordination number=8). For the $\mathrm{Pt}_{13}$ clusters, this coverage becomes as high

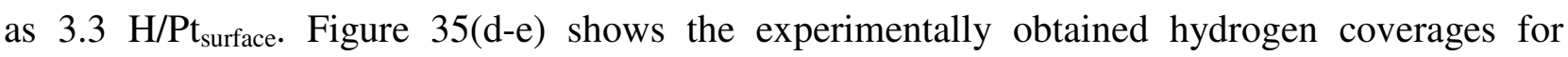
samples S1-S6 with Pt NPs with different size and shape [113]. XANES measurements are valuable for the study of the electronic properties of NPs, due to the sensitivity of this technique to the unoccupied electronic density of states [113; 277; 423-427]. Quantitative information on the amount of adsorbed hydrogen on Pt NPs could be extracted from the integrated area of the $\triangle X A N E S$ absorption peak [428-433]. Comparing the data acquired at $648 \mathrm{~K}$ in $\mathrm{H}_{2}$ and $\mathrm{He}$ environments, it was shown that the NPs are hydrogen-free at that temperature [18]. Therefore, the data acquired at that temperature were used as the hydrogen-free reference. For samples S1-S6 the shape of the NPs shown in Figure 35(e) are obtained using a combination of EXAFS and TEM measurements $[3 ; 111 ; 113]$. The number of $\mathrm{H}$ atoms per surface $\mathrm{Pt}$ atom was obtained from $\triangle \mathrm{XANES}$ measurements after normalization by the surface to bulk ratio in each NP sample as well as by the $\triangle \mathrm{XANES}$ area of a reference sample containing large NPs (140 atoms, S6), where a one $\mathrm{H} / \mathrm{Pt}$ ratio can be assumed. For all samples measured in $\mathrm{H}_{2}$, a progressive decrease in the hydrogen coverage was observed with increasing temperature. The maximum $\mathrm{H} / \mathrm{Pt}_{\text {surface }}$ coverage of $\sim 3$ is obtained at $\sim 200 \mathrm{~K}$ for S2. This is in good agreement with the theoretical calculations in Figure $35(a)$. 

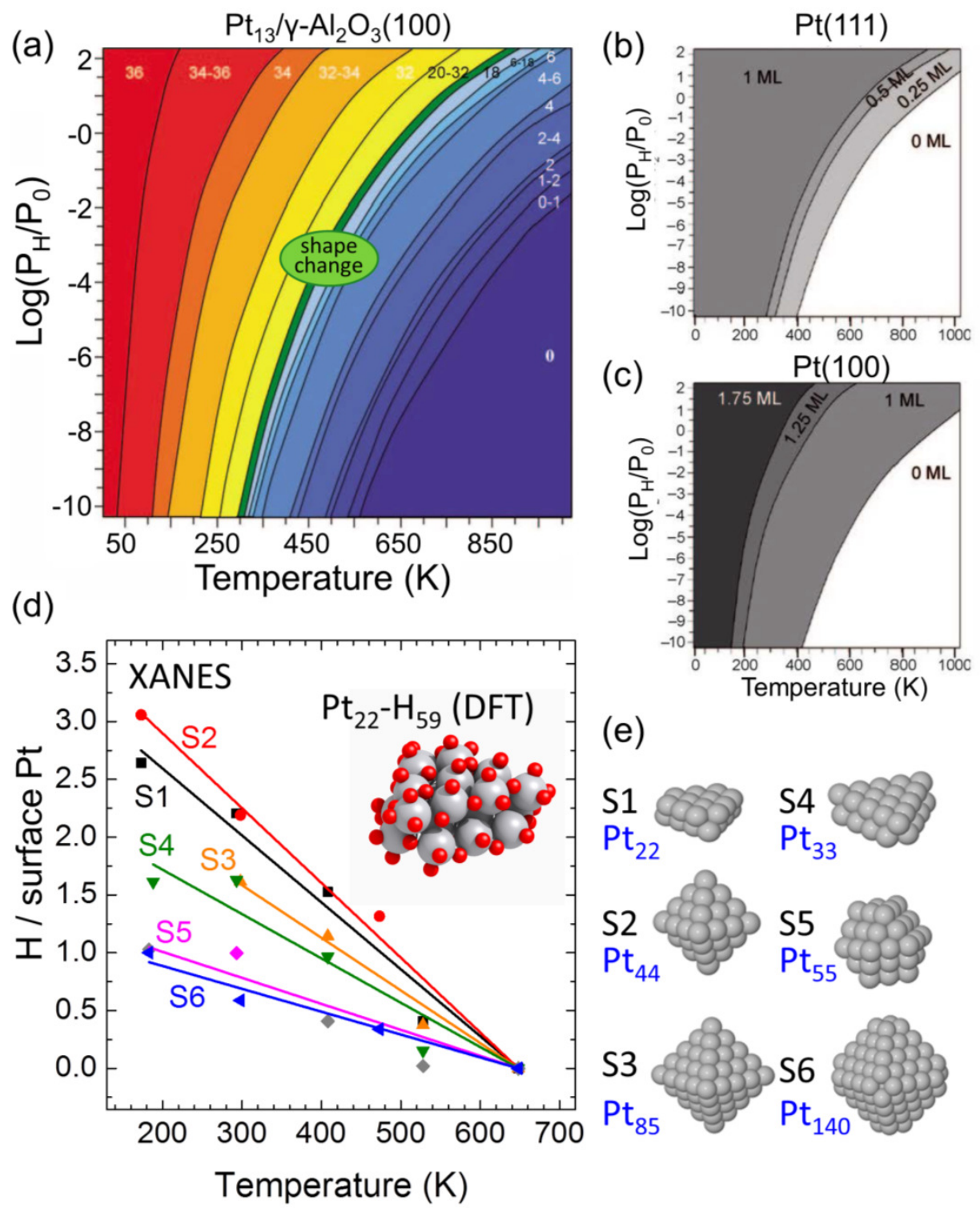

(e)

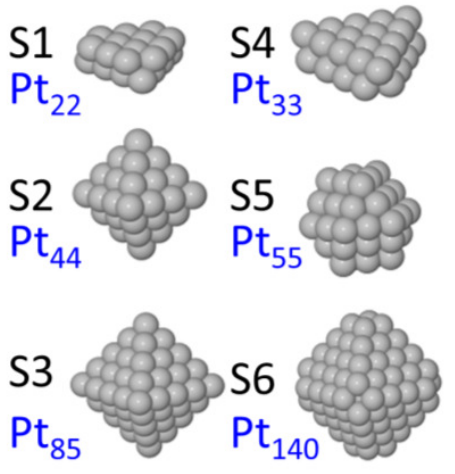

Figure 35: Thermodynamic diagrams depicting the most stable hydrogen coverage as a function of the temperature and the hydrogen partial pressure: (a) $\mathrm{Pt}_{13} / \gamma-\mathrm{Al}_{2} \mathrm{O}_{3}(100)$ (the number of hydrogen atoms per cluster is given in the corresponding zones). Similarly obtained $\mathrm{H}$ coverage for (b) $\operatorname{Pt}(111)$ and (c) $\operatorname{Pt}(100)$ surfaces. The hydrogen coverage of the most stable system in the given conditions is indicated in the corresponding zones in $\mathrm{ML}=$ monolayer units. (d) Area of the $\triangle$ XANES peak normalized by the ratio of surface to total number of atoms $\left(\mathrm{N}_{\mathrm{s}} / \mathrm{N}_{\mathrm{t}}\right)$ and by the area of the same peak of $\mathrm{S} 6\left(\mathrm{Pt}_{140}\right)$, measured at $183 \mathrm{~K}$, where $1 \mathrm{H}$ for each surface $\mathrm{Pt}$ atom is assumed. The $\mathrm{N}_{\mathrm{s}} / \mathrm{N}_{\mathrm{t}}$ normalization compensates for the fact that larger NPs have a lower number of atoms at their surface affected by $\mathrm{H}$ adsorption. The inset in (d) displays a DFT-obtained structure for $\mathrm{Pt}_{22^{-}}$ $\mathrm{H}_{59}$. (e) Model shapes for samples S1-S6 obtained from the combination of EXAFS and TEM analysis. (a-c) Adapted from Ref. [19], (d,e) were adapted from Ref. [113]. 
Another difference between terrace and step sites deals with the entropy, rather than the enthalpy. Beam surface scattering measurements combined with adsorption calorimetry experiments for $\mathrm{Pb}$ on $\mathrm{Mo}(100)$ by Starr et al. [434] also revealed large differences between the entropy of two different species adsorbed on steps and terraces. Such entropy differences were explained based on a 2D gas model for atoms adsorbed on the surface as compared to a 1D gas model for those on steps. In other words, adsorbates may show different behavior based on the adsorption sites, with some being more confined than others regarding atomic diffusion or vibration [434-436].

\section{(b) Effect on reactivity}

After much debate and many different proposed explanations for the high $\mathrm{CO}$ oxidation activity of the $\mathrm{Au} \mathrm{NP} / \mathrm{TiO}_{2}(110)$ system, the origin of such enhanced activity seems to be settled now. A review article by Lopez et al. [369] compiling available experimental and theoretical data on $\mathrm{CO}$ oxidation by gold concluded that the origin of the enhanced activity was mainly the abundance of low coordinated atoms on small NPs [437]. DFT calculations by Remediakis on $\mathrm{Au}_{10}$ NPs supported on $\mathrm{TiO}_{2}(110)$ provided two pathways for $\mathrm{CO}$ oxidation; one purely on $\mathrm{Au}$ NPs, and the other at the NP perimeter [438]. However, both pathways were found to rely on lowcoordinated $\mathrm{Au}$ atoms to stabilize the reactants [438]. Other studies had also confirmed the role of undercoordinated atoms as the essential factor in CO binding and oxidation over Au NPs [439].

Mostafa et al. showed that the onset temperature for the partial oxidation of 2-propanol to acetone carried out on $\mathrm{Pt} / \gamma-\mathrm{Al}_{2} \mathrm{O}_{3}$ correlates with the average number of missing bonds on the NP

surface, namely, the lower the average $\mathrm{CN}$ of the surface atoms, the lower will be the onset temperature (i.e. lower activation energy) [3]. In another study by Mistry et al. it was shown that the total oxidation of 2-butanol over $\mathrm{Pt} / \gamma-\mathrm{Al}_{2} \mathrm{O}_{3}$ follows the opposite trend due to oxygen poisoning that occurs more readily on low-coordinated atoms as a result of the higher binding energy of oxygen to those atoms [114].

In addition, the enhanced catalytic properties of some material systems (e.g. $\mathrm{Au} / \mathrm{TiO}_{2}$ ) have been also explained based on unique active sites at the NP-support interface with bifunctional properties rather than due to the altered electronic structure of low coordinated atoms or to charge transfer phenomena [383]. This topic will be discussed in more detail in section 3.3. 


\subsection{NP-support interaction and charge transfer}

Size-dependent NP/support interactions could play a significant role in the catalytic performance of the NPs. Such interactions affect (i) the electronic structure of the NPs through charge transfer processes, (ii) the crystalline and electronic structure of the NPs due to induced strain at the NP/support interface, or (iii) the properties of interfacial sites (metal atoms at the perimeter of the NP in contact with the support). Nonetheless, in most cases a combination of the above phenomena should be considered.

For supported metal NPs, one of the factors that would determine the thermal stability, the NP shape (wetting), and the adsorbate binding energy is the adhesion energy of the NPs to the support. For example, Figure 36 shows the heat of adsorption of Ag atoms to Ag NPs deposited on different supports as a function of the NP size [440]. As expected for small NPs, the heat of adsorption is smaller than for bulk systems since in average the adsorbed atoms are undercoordinated. In addition, for the same NP size, the heat of adsorption is higher for $\mathrm{CeO}_{\mathrm{x}^{-}}$ supported NPs as compared to those on $\mathrm{MgO}$. Furthermore, for $4 \mathrm{~nm}$-thick $\mathrm{CeO}_{\mathrm{x}}$ supports, the degree of reduction of the support affects the heat of adsorption, with the more reduced support (i.e. more vacancy trap sites) showing higher adhesion energy. The thickness of the oxide layer also seems to play a role: silver atoms in NPs smaller than $1.5 \mathrm{~nm}$ (30 atoms) on $\mathrm{CeO}_{1.9}(111)$ films are 20 to $50 \mathrm{~kJ} / \mathrm{mol}$ more stable when the film is only $1 \mathrm{~nm}$ thick (versus $4 \mathrm{~nm}$ ) [441; 442].

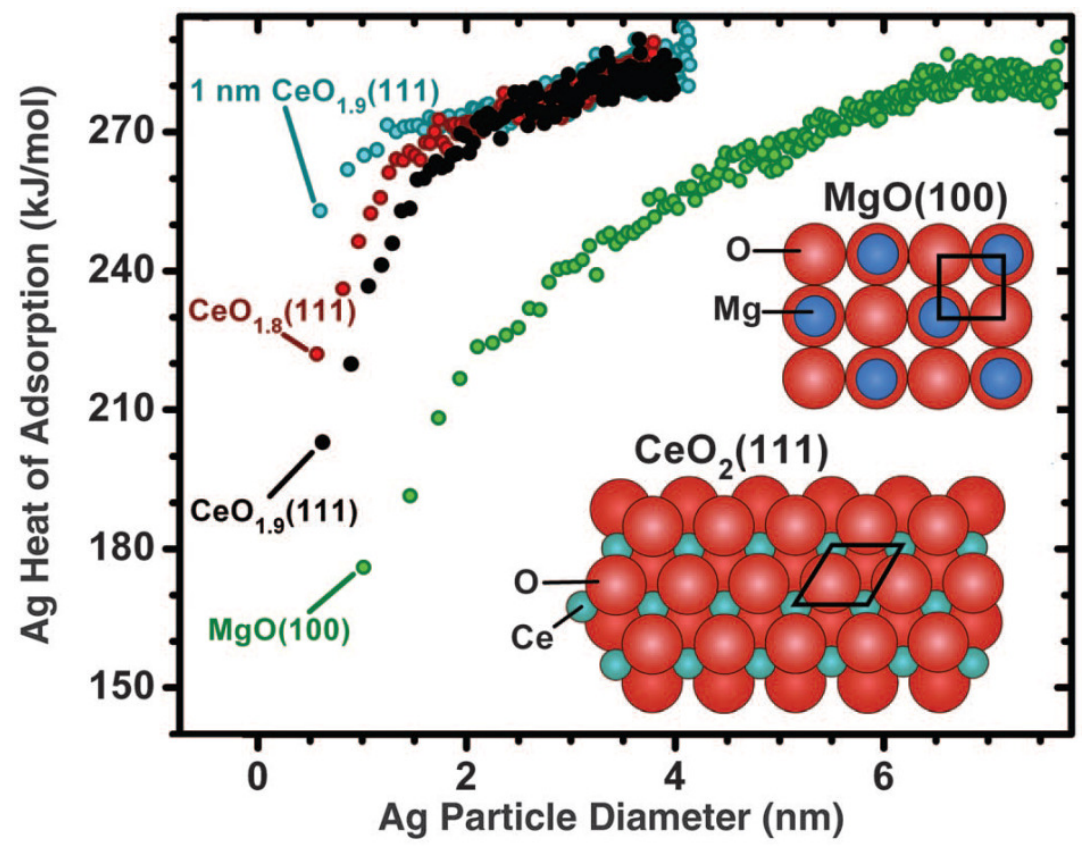


Figure 36: Measured heat of Ag atom adsorption versus the diameter of the Ag NP to which it becomes attached for $\mathrm{Ag}$ adsorption onto four different surfaces: two 4-nm $\mathrm{CeO}_{2}$ (111) films with different extents of surface reduction ( $\mathrm{x}=\sim 0.1$ and 0.2 in $\mathrm{CeO}_{2-\mathrm{x}}$ ) grown on $\operatorname{Pt}(111)$; a 1 nm-thick $\mathrm{CeO}_{1.9}$ (111) film grown on $\mathrm{Pt}(111)$; and a 4 nm-thick $\mathrm{MgO}(100)$ film grown on $\mathrm{Mo}(100)$. The inset shows structural models for perfect $\mathrm{CeO}_{2}(111)$ and $\mathrm{MgO}(100)$, with their unit cells indicated by black lines. Taken from Ref. [440].

It is known that strain may affect the electronic structure of metals and may thus change their catalytic activity $[183 ; 269 ; 270 ; 443]$. Figure 37 shows how the shape of Pd NPs supported on $\mathrm{MgO}(001)$ varies within different size regimes based on the differences in interfacial strain [196]. $\mathrm{Pd}$ and $\mathrm{MgO}(001)$ have an epitaxial interface with a misfit of $7.6 \%$. Further, such epitaxiallyinduced strain can propagate towards the surface of small NPs, alter their surface energy and energetics, and ultimately lead to a change in their shape [444; 445]. Small NPs (<3nm) may accommodate the interfacially-induced strain through the entire NP. For larger NPs the accumulated strain results in distortions only in the first three atomic layers in contact with the support, while the upper part of the NPs may remain strain-free [196; 260; 446; 447]. Larger NPs $(10-15 \mathrm{~nm})$ release the interfacial strain through lattice dislocations. Such size-dependent strain distribution results in modified surface energy anisotropy $\left(\sigma_{100} / \sigma_{111}\right)$, or in other words, in different NP shapes [196]. Similar findings for Pt NPs supported on $\mathrm{TiO}_{2}(110)$ have also been reported, with small NPs (<3000 atoms) adopting pyramid-like shapes with a Pt(100) interfacial facet, NPs with intermediate size (2000-6000 atoms) adopting a hexagonal shape with $\operatorname{Pt}(111)$ at the interface, and large NPs (>5000 atoms) adopting a wedge-top shape with $\mathrm{Pt}(110)$ at the $\mathrm{Pt}$ $\mathrm{NP} / \mathrm{TiO}_{2}(110)$ interface [100]. 


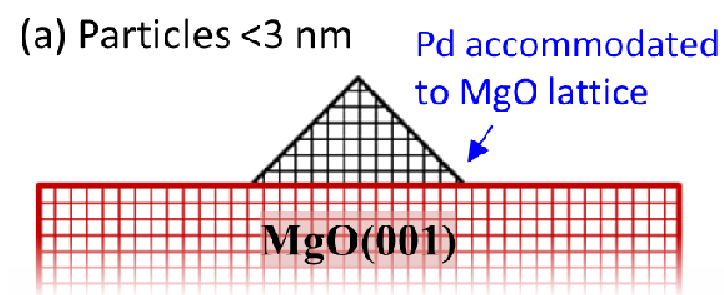

(b) Particles 4-5 nm

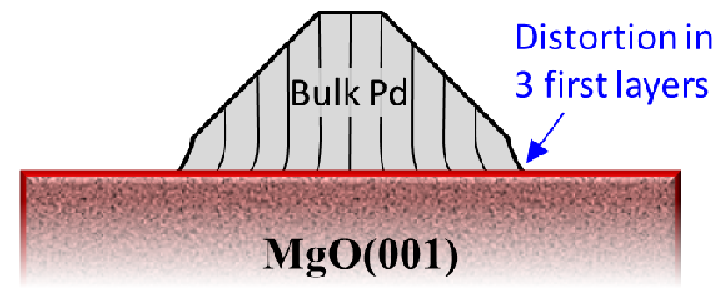

(c) Particles 10-15 nm

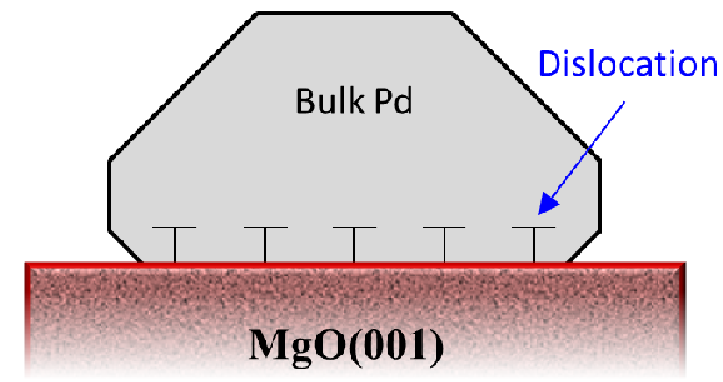

Figure 37: Schematic of the NP-support interface for NPs with different sizes and shapes: (a) NPs smaller than $3 \mathrm{~nm}$ and (b) 4-5 nm NPs, both prepared by UHV condensation or wet impregnation on $\mathrm{MgO}$ microcubes; (c) 10-15 nm NPs prepared by UHV condensation on MgO single crystals. Source Ref. [196]

In addition, the shape of the NPs could also be tuned based on NP-support charge transfer phenomena induced by the presence of dopants or inpurities in the support [448]. For instance, a small amount of $\mathrm{Mo}$ in a $\mathrm{CaO}$ film could change the shape of $\mathrm{Au}$ NPs supported on $\mathrm{CaO}$ from $3 \mathrm{D}$ on the undoped support to 2D on the Mo-doped substrate [448].

Figure 38 displays DFT results for Au clusters supported on $\mathrm{MgO}(100)$ as well as on $\mathrm{MgO} / \mathrm{Mo}(100)$ [449]. Different $\mathrm{Au}_{\mathrm{n}}$ clusters $(\mathrm{n}=8,16,20)$ are considered with both, 3D and planar structures, Figure 38(a-f). Based on DFT calculations, on $\mathrm{MgO}(100)$ the 3D structure is preferred for $\mathrm{Au}_{8}$ and $\mathrm{Au}_{20}$, while for $\mathrm{Au}_{16}$ the planar structure is more stable. In contrast, for the $\mathrm{MgO} / \mathrm{Mo}(100)$ system, planar structures (maximal wetting of the surface), are found to be the most stable configurations, for all $\mathrm{Au}_{\mathrm{n}}$ clusters with much larger energy gain over the 3D shapes as compared to $\mathrm{Au}_{\mathrm{n}} / \mathrm{MgO}(100)$ system. The higher adhesion energy per atom in contact with the 
support is clearly shown in Figure 38(g). Furthermore, for $\mathrm{Au}_{8}$ clusters adsorbed on $\mathrm{MgO} / \mathrm{Mo}(100)$, the adhesion energy per contact atom $\left(\tilde{\mathrm{E}}=\mathrm{E}_{\mathrm{adh}} / \mathrm{n}_{\text {cont }}\right)$ decreases from $0.7 \mathrm{eV}$ to 0.3 $\mathrm{eV}$ as the thickness of the oxide film increases from 2 layers to 7 layers, Figure 38(h). A clear correlation is also observed between the amount of charge transferred from the metal to the cluster-oxide interface and the $\mathrm{MgO}$ thickness [inset in Figure 38(h)], demonstrating the decay of the metal states penetrating through the oxide film by increasing the oxide thickness [449].
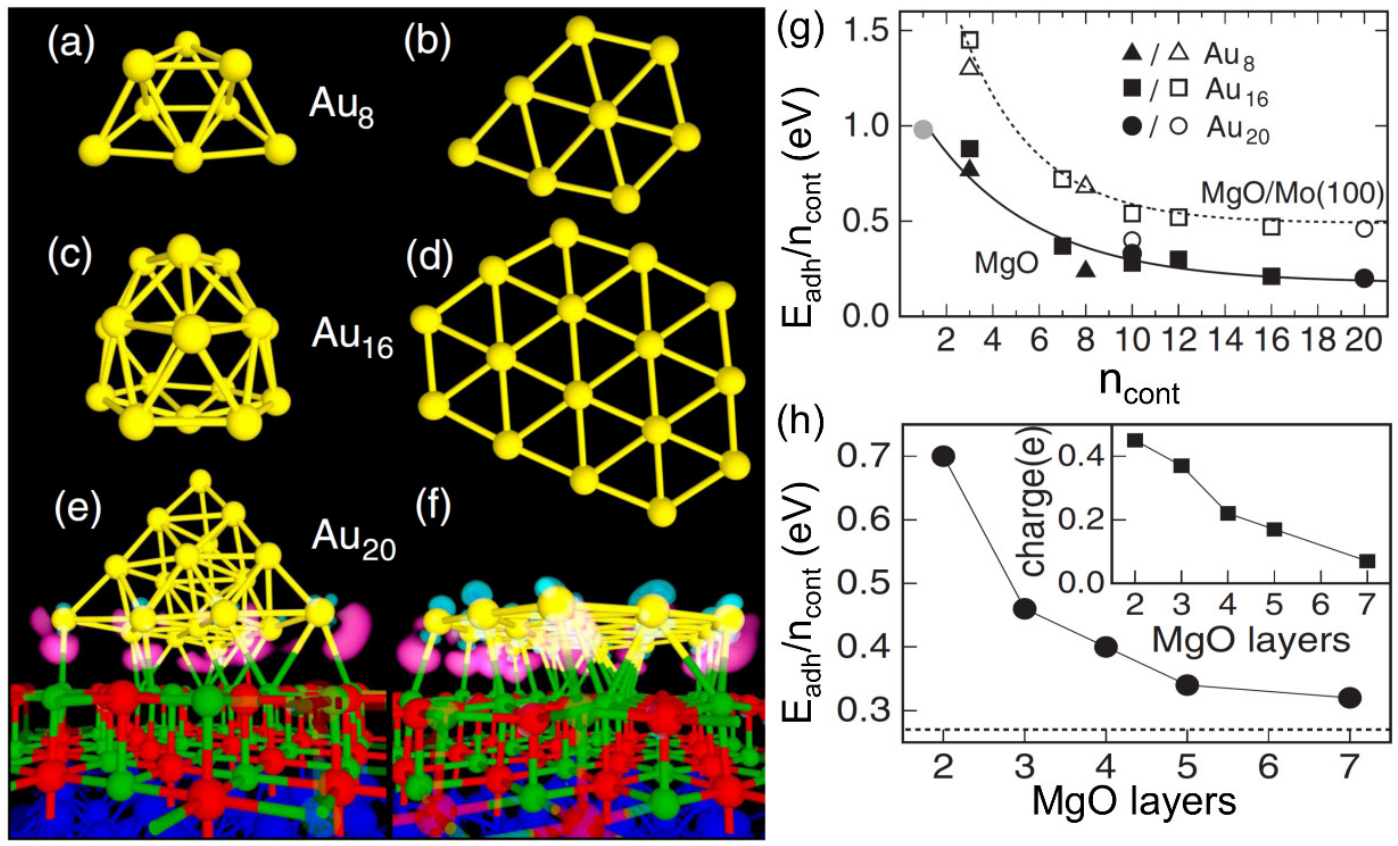

Figure 38: Relaxed atomic configurations of isolated unsupported $\mathrm{Au}_{\mathrm{n}}$ cluster isomers (a-d) and their modifications upon adsorption on $\mathrm{MgO} / \mathrm{Mo}(100)$, (e,f). The structures in $(\mathrm{a}, \mathrm{c}, \mathrm{e})$ are $3 \mathrm{D}$ and those in (b,d,f) are planar. For the $\mathrm{Au}_{20}$ clusters (e) and (f ) adsorbed on $\mathrm{MgO} / \mathrm{Mo}(100)$, the accumulated charge density at the cluster-oxide interface is also displayed, with charge accumulation shown in pink, and charge depletion in light blue. Yellow, green, red, and dark blue spheres correspond to $\mathrm{Au}, \mathrm{Mg}, \mathrm{O}$, and Mo atoms, respectively. (g) Adhesion energy per contact atom $(\tilde{\mathrm{E}})$ of $\mathrm{Au}_{\mathrm{n}}$ cluster isomers on $\mathrm{MgO}(100)$ (filled symbols) and on a two-layer $\mathrm{MgO}$ thin film supported on Mo(100) (empty symbols), plotted vs the number of gold atoms in contact with the oxide surface $\left(\mathrm{n}_{\text {cont }}\right)$. Lines are added to guide the eye. Also included is the adhesion energy for a single $\mathrm{Au}$ atom on $\mathrm{MgO}$ (gray circle) [450]. The adhesion energy of a single $\mathrm{Au}$ atom on $\mathrm{MgO} / \mathrm{Mo}(100)$ falls on the continuation of the dashed line (not shown) [450]. (h) Adhesion energy per contact atom $\left(\mathrm{n}_{\text {cont }}=8\right), \tilde{\mathrm{E}}$, of planar $\mathrm{Au}_{8}$ clusters adsorbed on $\mathrm{MgO} / \mathrm{Mo}(100)$ plotted vs the thickness (number of layers) of the $\mathrm{MgO}$ thin film. The inset shows the accumulated charge at the cluster-oxide interface plotted vs the number of $\mathrm{MgO}$ layers. The dashed line denotes $\tilde{\mathrm{E}}$ for a planar $\mathrm{Au}_{8}$ cluster on a nondefective $\mathrm{MgO}(100)$ surface. Adapted from Ref. [449]. 
Among the different surface sites, special attention should be paid to perimeter atoms which are in direct contact with the support $[451 ; 452]$. Although all the atoms at the NP-support interface are subject to the largest support-induced effects, most of such atoms are in fact buried under the NP and only those at the perimeter (i.e. dual sites) can directly participate in catalytic reactions. One of the most extensively studied systems with respect to metal/support interactions is $\mathrm{Au} / \mathrm{TiO}_{2}[358 ; 453-458]$. The crucial role of perimeter $\mathrm{Au}$ atoms in this system has been elucidated for $\mathrm{CO}$ oxidation [453-455], $\mathrm{H}_{2}$ oxidation [456], methanol decomposition [457], and methanol oxidation [458] using experimental and theoretical calculations. Interfacial dual sites have been also shown to play a pivotal role in other systems such as $\mathrm{H}_{2}$ activation on $\mathrm{Ag} / \theta-\mathrm{Al}_{2} \mathrm{O}_{3}(110)$ [459], $\mathrm{CO}$ oxidation over $\mathrm{Au} / \mathrm{CeO}_{2}$ [460], and water gas shift reaction on $\mathrm{Pt} / \mathrm{Mo}_{2} \mathrm{C}$ [461].

In some cases the perimeter sites display enhanced reactivity due to charge transfer phenomena that may facilitate certain reaction steps with high activation energy. In other cases they simply provide the possibility for the reactants to form bonds to both, atoms within the NP and those at the support. For instance, sub-nanometer alumina-supported Ag clusters have been found to be active for low temperature direct propylene epoxidation to propylene oxide by molecular oxygen [313]. Figure 39 shows the reaction energy profiles for two reaction pathways, namely $\mathrm{O}_{2}$ dissociation (i) on top of the NP, and (ii) at a NP-support interfacial site [462]. The energy barrier defined based on the energy of the transition state is found to be much smaller for $\mathrm{O}_{2}$ dissociation at the interface $(0.16 \mathrm{eV})$ as compared to the pathway in which the $\mathrm{O}_{2}$ dissociates on top of the NP $(0.46 \mathrm{eV})$ [462]. 


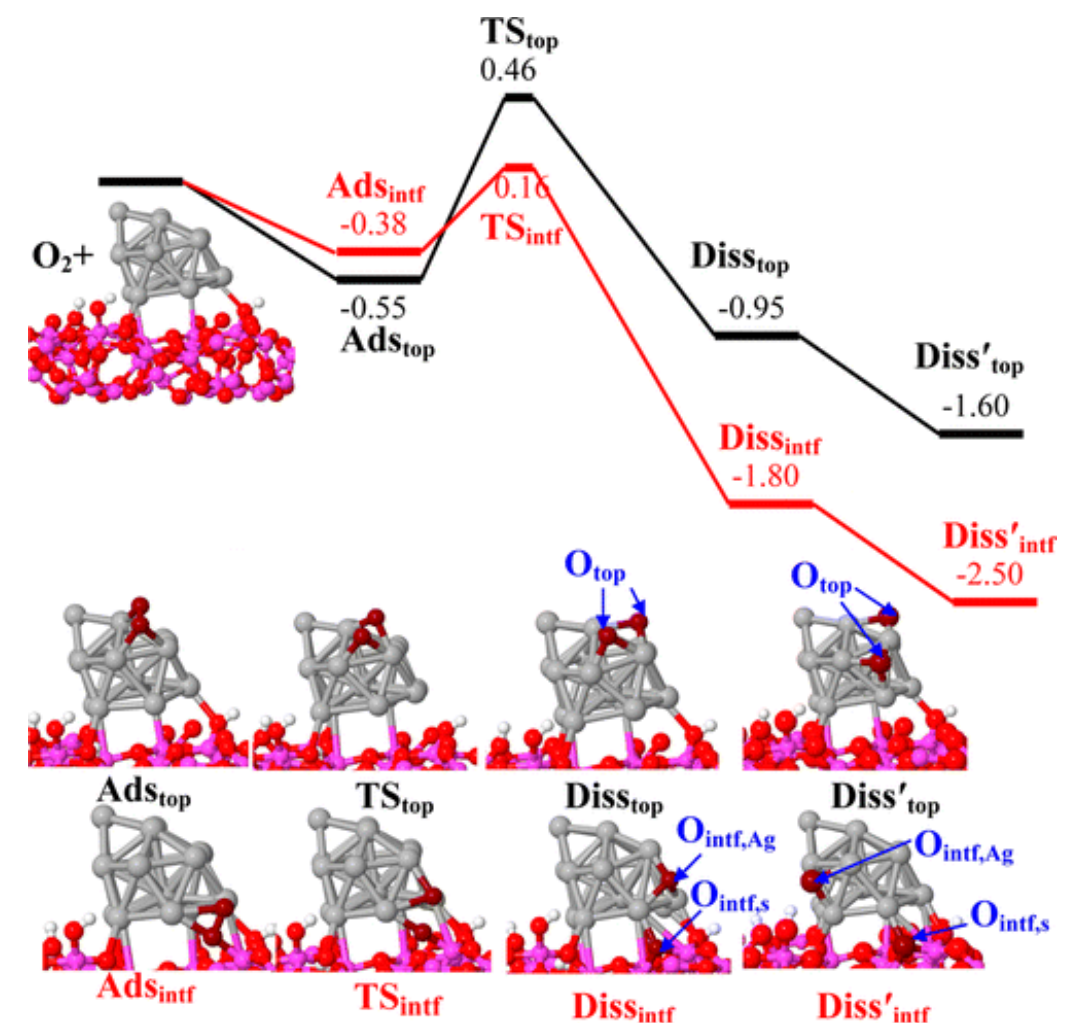

Figure 39: Reaction energy profile for $\mathrm{O}_{2}$ dissociation on alumina-supported $\mathrm{Ag}_{19}$ interfacial (red) and top sites (black). Structures of $\mathrm{O}_{2}$ adsorption (Ads), dissociation transition state (TS), and product of dissociation (Diss) are shown. More stable structures with two oxygen atoms apart from each other after $\mathrm{O}_{2}$ dissociation are also shown (Diss'). Silver, oxygen, aluminum, and hydrogen atoms are shown in silver, red, magenta, and white, respectively. The two oxygen atoms from the $\mathrm{O}_{2}$ molecule are shown in dark red for clarity. Adapted from Ref. [462].

In addition, it has been shown in several examples that some of the elements in the support lattice (e.g. oxygen) could directly participate in the reaction [199; 453; 463-465]. For instance, using DFT calculations, it was suggested that $\mathrm{Au}_{12}$ clusters supported on stepped $\mathrm{CeO}_{2}$ could facilitate $\mathrm{CO}$ oxidation by directly using lattice oxygen atoms at $\mathrm{CeO}_{2}$ step edges as an oxygen source, following the Mars-van Krevelen (MVK) mechanism [199]. For Pt, Pd and Ni NP catalysts supported on $\mathrm{CeO}_{2}$ it also has been shown that during $\mathrm{CO}$ oxidation oxygen is provided by the support. Interestingly, the scaling factors (size dependence) observed for these samples were consistent with the scaling factors calculated when the involvement of perimeter sites was considered, and therefore, the size-effect observed was assigned to the fact that smaller NPs feature a larger fraction of perimeter surface sites [464]. Another approach to study the role played by the perimeter sites is to consider reverse catalysts [466]. For instance, the promoting effect of an iron oxide support $\left(\mathrm{Fe}_{2} \mathrm{O}_{3}\right)$ on the $\mathrm{CO}$ oxidation activity of $\mathrm{Au}$ NPs has been shown in several 
studies [467-469]. Furthermore, an inverse system in which $\mathrm{Fe}_{2} \mathrm{O}_{3}$ islands are formed on $\mathrm{Au}(111)$ has also been considered [466]. In the latter example, an increase in the CO oxidation rate was demonstrated with increasing $\mathrm{Fe}_{2} \mathrm{O}_{3}$ coverage until a coverage of 0.5 is reached. Further increasing the coverage above 0.5 results in a decline of the activity until it reaches zero when a complete coverage of the $\mathrm{Au}(111)$ surface is achieved [466]. This observation is agreement with a reaction mechanism in which the perimeter sites are the main active sites.

Other processes that also involve perimeter sites are spillover phenomena in which the reactant species adsorbed on the support react with those adsorbed on the NPs. These processes require the transport of reactants or reaction intermediates toward or away from the NPs, with the perimeter sites acting as gateways. For instance, for the oxidation of CO over large Pd NPs (> $7 \mathrm{~nm})$ on $\mathrm{MgO}(100)$ it has been shown that the spillover of $\mathrm{CO}$ from the support to the NPs is responsible for the 50\% increase in turnover rate measured [470]. In another example, the higher activity of $\mathrm{Pt} /\left(\mathrm{Al}_{2} \mathrm{O}_{3}-\mathrm{SiO}_{2}\right)$ catalysts for benzene and toluene hydrogenation is explained based on the adsorption of aromatic hydrocarbons on the support and their reaction with activated hydrogen that is spilled-over from the Pt NPs [471]. Photocatalytic reforming of methanol on $\mathrm{Pd} / \mathrm{TiO}_{2}$ proceeds in steady state only in the presence of light [472]. This is explained based on CO poisoning of $\mathrm{Pd}$ sites that can be lifted by the photogenerated oxygen in $\mathrm{TiO}_{2}$ [472]. In above cases the support plays an active role in the catalytic reaction in addition to its effect in terms of altering the NP properties.

We should also have in mind that the NPs also could affect the support in return by catalytically reducing it or through spill-over phenomena [205; 473; 474]. Such interactions are also found to be size- and shape-dependent due to the enhanced catalytic properties of small NPs as well as their size-dependent stability. For instance, while small PVD-grown NPs tend to decorate $\mathrm{TiO}_{2}(110)$ steps $[27 ; 146 ; 475 ; 476]$, larger micelle-synthesized Pt NPs ( 3 nm) have been shown to be immobile up to significant temperatures $\left(1060^{\circ} \mathrm{C}\right)$, with $\mathrm{TiO}_{2}$ steps reconfiguring around the NPs, and the NPs helping to stabilize certain steps, leading to the formation of NPpinned $\mathrm{TiO}_{2}$ stripes $[205 ; 207]$. Figure 40 shows a schematic of this phenomenon as well as an example of STM images of micellar Pt NPs/TiO $2(110)$. $\mathrm{TiO}_{2}$ reduction upon high temperature annealing in vacuum is a well-known phenomenon that occurs at the surface and propagates into the bulk. Such reduction has been evidenced by the apparent loss of material and recession of the step edges $[477 ; 478]$. The released oxygen escapes to vacuum while the $\mathrm{Ti}$ cations $\left(\mathrm{Ti}^{3+}\right)$ diffuse 
inward into the bulk, and by doing so, the top most layers of the $\mathrm{TiO}_{2}$ substrate gradually disappear [477; 478]. Interestingly, this gradual disappearance of $\mathrm{TiO}_{2}$ rows starts at $[1 \overline{1} 0]$ step edges and follows a chain reaction that proceeds along $\mathrm{TiO}_{2}$ rows in the [001] direction [478]. Pt and Au NPs could stabilize [110] steps and prevent the $\mathrm{TiO}_{2}$ from dissociating, resulting in the formation of long $\mathrm{TiO}_{2}$ nanostripes [205].

In another study it was shown that Au NPs supported on $\mathrm{SiO}_{2} / \mathrm{Si}(100)$ could destabilize the $\mathrm{SiO}_{2}$ thin film support and catalytically reduce it to $\mathrm{Si}$ upon annealing in UHV at high temperature $\left(>700^{\circ} \mathrm{C}\right)$. Figure $40(\mathrm{~b})$ shows the schematic of this phenomenon as well as an AFM image of a $\mathrm{Au}$ NP sitting inside a $\mathrm{SiO}_{2}$ void region created by the NP. After the initial reduction induced by the NP, the $\mathrm{SiO}_{2}$-depleted region at the NP-support interface could independently grow laterally around the NP nucleation point.
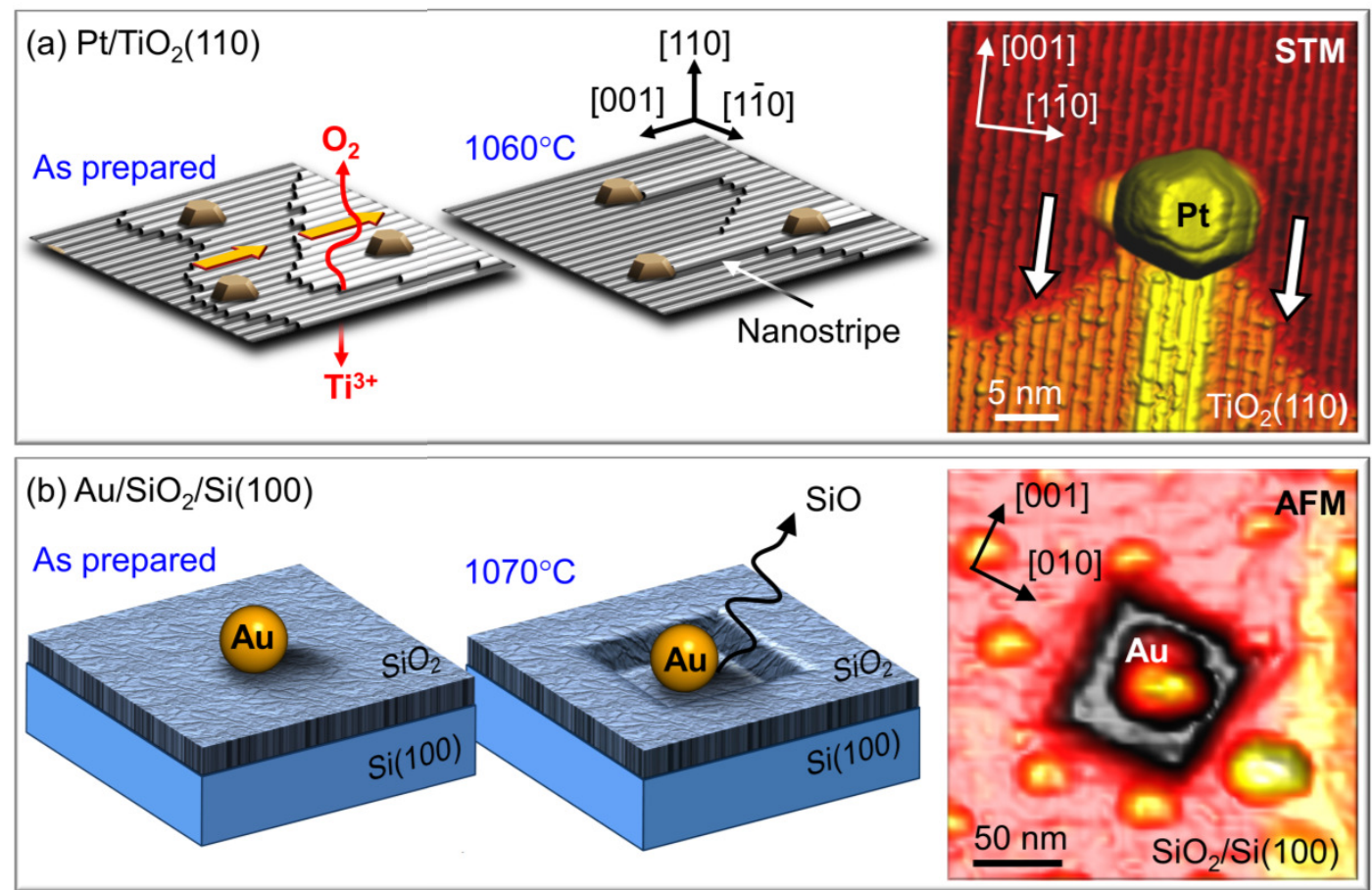

Figure 40: (a) Schematic (left) and STM image (right) of the stabilization of $\mathrm{TiO}_{2}$ steps mediated by Pt NPs. The reduction of $\mathrm{TiO}_{2}$ occurs mainly at step edges with [001] step edges being the most stable. All step edges other than [001] lose oxygen to vacuum, while $\mathrm{Ti}^{3+}$ diffuses inward as interstitial species. The step edges protected by Pt NPs remain stable and the recession of other steps result in the formation of nanostripes as shown in the schematic [205]. (b) Schematic (left) and AFM image (right) of the Au-NP induced catalytic reduction of $\mathrm{SiO}_{2}$ thin films reaching the $\mathrm{Si}(100)$ substrate underneath. $\mathrm{SiO}$ species produced from $\mathrm{SiO}_{2}$ reduction underneath the $\mathrm{Au}$ NPs are volatile and can escape to vacuum. Subsequently, the diffusion of Si adatoms on top of bare $\mathrm{Si}(100)$ allows the $\mathrm{Si}+\mathrm{SiO}_{2} \rightarrow 2 \mathrm{SiO}$ reaction to occur on the edge of the void region, resulting in the growth of the void region around the NP independently of the initial NP nucleation [473]. 
In the case of highly-oriented pyrolytic graphite (HOPG), the interaction between the NPs and the support is twofold: (i) the support affects the NPs by stabilizing them at step edges and defect sites [133], and (ii) NPs could generate defects on the support surface and catalytically etch it under for example an oxygen environment [133; 479]. A complete basal plane of HOPG is very stable due to the saturation of all carbon bonds. Therefore, at temperatures below $700^{\circ} \mathrm{C}$, the oxidation of the original basal plane atoms remains unaffected, and the etching of the surface is initiated mainly at defects on the basal plane, or edge atoms on monoatomic steps [133]. In addition, HOPG basal planes have a very weak interaction with NPs and a very small diffusion barrier in different surface directions which tend to result in NP aggregation. Limat et al. have used small $\mathrm{Au}_{5} \mathrm{NPs}$ to create defects on HOPG as the nucleation point for the growth of larger pits [133; 135]. The implantation of mass selected $\mathrm{Au}_{5}$ clusters (impact energy of $3 \mathrm{kV}$ ) results in surface defect formation and $\mathrm{C}$ dangling bonds at the defect edges. An oxygen treatment could etch the defect sites growing them into larger pits. Next, the deposition of Au into the prepared HOPG surface results in size-controlled Au NPs that are stabilized by the step edges at the pits perimeter. This is an example where the Au NPs were used to modify the substrate and eventually were stabilized on the modified surface.

Despite the examples above, it needs to be recognized that the support effect is fundamentally size-dependent. Small 2D (flat) NPs have a higher surface to bulk ratio and therefore, they have more atoms at the NP-support interface. Moreover, the atomic population at the NP perimeter has been shown to be a key player for the understanding of the activity and selectivity of numerous catalytic reactions [480; 481], and such parameter has a 1/r dependency. Also, the strain induced by the support propagates differently through the NP depending on the NP size and shape. Furthermore, charge transfer phenomena at the NP/support interface may affect the entire NP for small NPs. Therefore, it is usual to have size-dependent activity whenever the NP-support interaction also plays a crucial role.

The most straight forward method to consider the support effect for different support materials is to prepare similar NPs (analogous size) on different supports. We should have in mind that some NP synthesis methods may not be appropriate for this purpose. For instance, physical vapor deposited (PVD) NPs prepared on different supports may turn out to have very different size and density depending on the diffusion barrier of adatoms on the support surface, with a higher 
diffusion barrier resulting in a larger number of small NPs and vice versa [27]. Other synthesis methods such as deposition-impregnation suffer similar problems, with distinct NP size, shape, and density distributions on different supports. However pre-formed isolated NPs such as those prepared by the inverse micelle method are excellent for this purpose, since the NPs have no direct interaction with the support until the protecting polymers are removed.

\section{(a) Adsorption properties}

STM and TPD studies on $\mathrm{Au} \mathrm{NP}_{\mathrm{S}}$ deposited on well-ordered alumina and iron oxide films revealed that the $\mathrm{CO}$ binding energy is essentially independent of the support studied, and therefore, support effects seen in $\mathrm{CO}$ oxidation reactions arise from the interaction of oxygen rather than $\mathrm{CO}$ with these catalysts [12].

While it appears that the adsorption of CO on Au NPs is not very sensitive to the support material $\left(\mathrm{Al}_{2} \mathrm{O}_{3}, \mathrm{FeO}\right.$, and $\mathrm{Fe}_{3} \mathrm{O}_{4}$, films) [12], a stark difference has been reported for Pd NPs deposited on $\mathrm{MgO}(100)$ and $\mathrm{Fe}_{3} \mathrm{O}_{4}(111)$ [147; 241; 243; 261; 264]. Based on TPD experiments, the $\mathrm{CO}$ binding energy for Pd NPs on $\mathrm{MgO}(100)$ showed a normal behavior, with stronger $\mathrm{CO}$ binding with decreasing NP size $(33 \mathrm{~kJ} / \mathrm{mol}$ decrease in initial binding energy by increasing the NP size from 2 to $5 \mathrm{~nm}$ ) [261]. In contrast, for the $\mathrm{Pd} / \mathrm{Fe}_{3} \mathrm{O}_{4}(111)$ system, the opposite trend was observed, with the heat of adsorption increasing about $30 \mathrm{~kJ} / \mathrm{mol}$ with increasing NP size from 2 to $4 \mathrm{~nm}[241 ; 264]$. As it was explained in section 3, this phenomenon is likely at least partially related to the strong interaction of the NPs with the $\mathrm{Fe}_{3} \mathrm{O}_{4}$ support.

DFT calculations on Au NPs have shown an enhanced binding of adsorbates on negatively charged NPs [376]. Such enhancement suggest that electrostatic charging of clusters supported on an oxide surface could affect the catalytic activity substantially [376]. Interestingly, a net charge transfer to the $\mathrm{Au}$ NPs has been reported for $\mathrm{Au} / \mathrm{TiO}_{2}$ and $\mathrm{Au} / \mathrm{MgO}$, which could be partially responsible for the high activity of these systems [376; 482-485]. Other studies also suggested that a positive charge transfer to Au NPs could also help their catalytic activity [369].

However, NP-support interaction may compromise or even entirely block the free surface of the NPs [486-488]. In some cases, the "strong metal-support interaction" (SMSI) could result in catalytic deactivation through the encapsulation of the NPs by a thin film of support material blocking the NP surface entirely. Such phenomena have been reported for a number of systems, including $\mathrm{Rh} / \mathrm{TiO}_{2}$ [489-491], $\mathrm{Pd} / \mathrm{TiO}_{2}$ [492-494], $\mathrm{Pt} / \mathrm{Fe}_{3} \mathrm{O}_{4}$ [10; 253; 495], $\mathrm{Pt} / \mathrm{TiO}_{2}$ and $\mathrm{Pt} / \mathrm{CeO}_{2}$ in 
reducing environments [476; 487; 496-499]. Figure 41(a) shows the sticking probability of CO on $\mathrm{Pd} / \mathrm{TiO}_{2}(110)$ measured at $223 \mathrm{~K}$ which drops very quickly after pre-annealing at/above $650 \mathrm{~K}$ due to Pd encapsulation by $\mathrm{TiO}_{\mathrm{x}}$. STM images shown in Figure 41(b,c) show an atomically-resolved image of the TiOx encapsulating layer formed on top of flat Pd NPs after annealing in vacuum at $973 \mathrm{~K}$. In Figure 41, the $\mathrm{Pt} / \mathrm{TiO}_{2}$ system is investigated by low energy ion scattering (LEIS) and STM [499]. The Ti signal that is prominent in the as prepared sample disappears entirely after 25 ML Pt deposition, Figure $41(\mathrm{~d})$. However, after high temperature annealing in vacuum $\left(700{ }^{\circ} \mathrm{C}\right.$ for $5 \mathrm{~min}$, and to $500{ }^{\circ} \mathrm{C}$ for $30 \mathrm{~min}$ ), enhanced nanoparticle coarsening results in larger clusters with the $\mathrm{TiO}_{2}$ support being exposed in between the NPs [Figure 41(e)], and therefore, the Ti signal is expected to appear again in the LEIS data. Nevertheless, such high temperature annealing also was also found to result in the complete disappearance of the Pt signal due to $\mathrm{TiO}_{\mathrm{x}}$ encapsulation as shown in the top panel of Figure 41(d) [499]. The atomically resolved structure of the $\mathrm{TiO}_{\mathrm{x}}$ encapsulating layer is visible in Figure 41(e-f). 


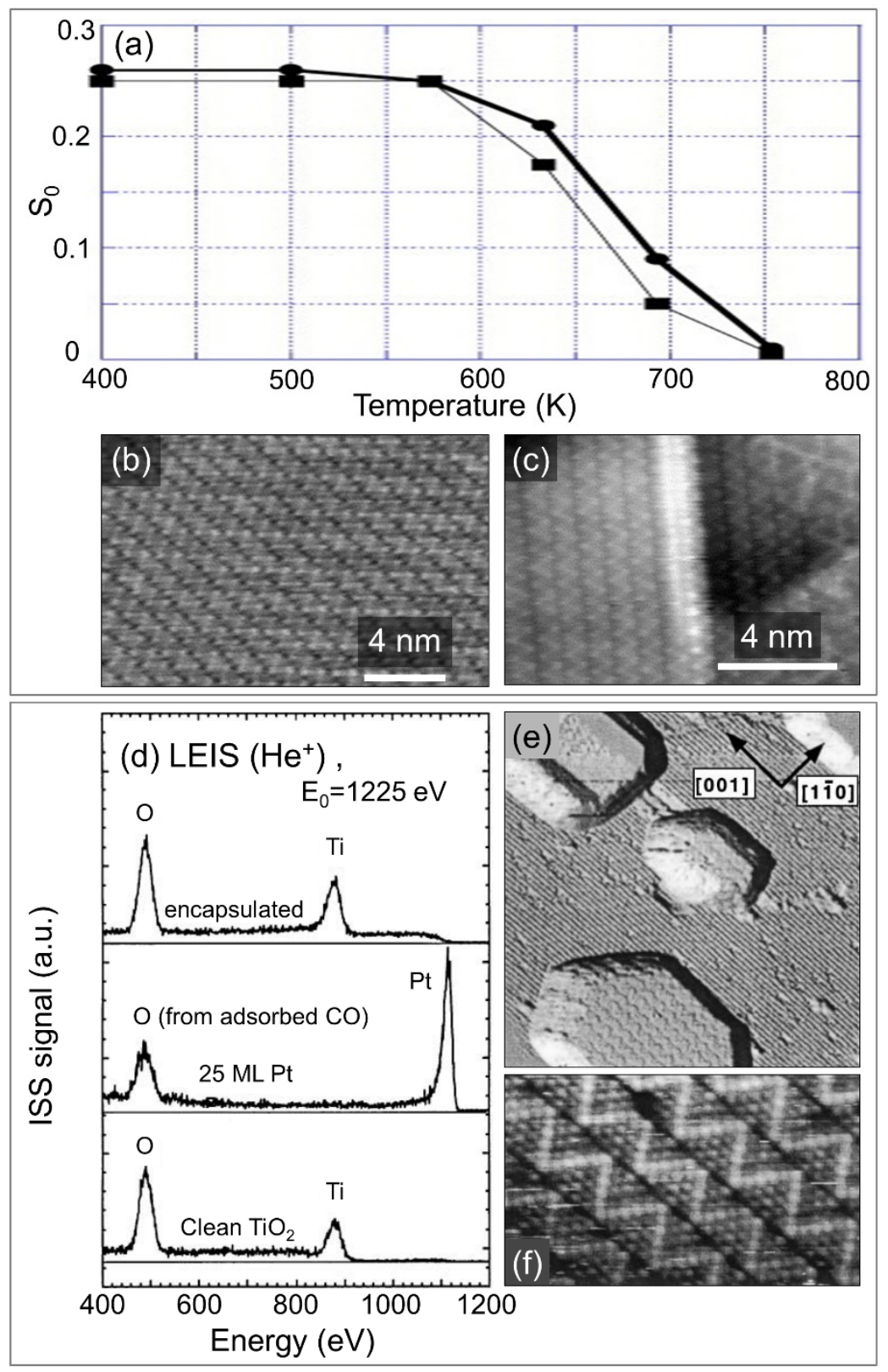

Figure 41: (a) Variation of the initial sticking probability of $\mathrm{CO}$ (that is the $\mathrm{S}$ value at zero coverage of $\mathrm{CO}\left(\mathrm{S}_{0}\right.$, circles) and saturation coverage (squares), on $\mathrm{Pd} / \mathrm{TiO}_{2}(110)$ as a function of the sample pre-annealing temperature. The sticking was measured at $223 \mathrm{~K}$ using a molecular beam of CO [492]. (b,c) STM images of the detailed surface structure of $\mathrm{TiO}_{2}(110)$-supported Pd films acquired after annealing at $973 \mathrm{~K}$ [493]. Two zigzag (b,c) and pinwheel (c) structures could be seen for the encapsulating $\mathrm{TiO}_{x}$ thin film [493]. (d) Low-energy $\mathrm{He}^{+}$ion scattering (LEIS) spectra of (bottom) the clean $\mathrm{TiO}_{2}(110)$ surface, (center) the same surface after evaporation of 25 monolayers $\mathrm{Pt}$ at room temperature, and (top) after a high-temperature treatment $\left(700{ }^{\circ} \mathrm{C}\right.$ for 5 $\mathrm{min}$, and to $500{ }^{\circ} \mathrm{C}$ for $30 \mathrm{~min}$ ) causing encapsulation [499]. (e-f) STM results after the hightemperature treatment [499]. Clusters are approximately $200 \AA$ wide and $40 \AA$ high. Most clusters show hexagonal shape [top facet $\mathrm{Pt}(111)$ ] elongated along the substrate [001] direction. (e) Smallscale image (500 $\mathrm{\AA} 500 \AA$ ), filtered to show the structure of the encapsulation layer $\mathrm{Pt}(111)$ top 
facet. (f) Atomic-resolution image of an encapsulated hexagonal cluster [499]. Adapted from Refs. [492; 493; 499].

\section{(b) Effect on reactivity}

Comparing the $\mathrm{CO}$ oxidation activity of $\mathrm{Au}$ NPs supported on $\gamma-\mathrm{Al}_{2} \mathrm{O}_{3}, \mathrm{ZrO}_{2}$, and $\mathrm{TiO}_{2}$ the best activity was obtained for $\mathrm{TiO}_{2}$, and the worst for $\gamma-\mathrm{Al}_{2} \mathrm{O}_{3}$ [500]. TEM measurements provided a similar NP size on all supports (as prepared size of 2.6, 2.9 and $2.9 \mathrm{~nm}$, respectively). Using XPS, NP charging was found to occur, but ruled out as the origin of the superior activity of $\mathrm{TiO}_{2}$ and $\mathrm{ZrO}_{2}$, and it was suggested that the support should be directly involved in the reaction [500].

For $\mathrm{CO}$ oxidation reactions over $\mathrm{Au} \mathrm{NP} / \mathrm{MgO}$, the interfacial sites at the NP perimeter were found to play an essential role in the reaction kinetics [480; 481]. For such reactions, small NPs with higher population of perimeter atoms would be favored. A similar mechanism has been suggested for $\mathrm{Au} \mathrm{NPs} / \mathrm{TiO}_{2}[6 ; 501 ; 502]$. A weakly binding support would prevent NP wetting, minimizing thus the number of perimeter interfacial atoms.

In some cases the catalytic activity is the result of the cooperative work from the metal NPs per se as well as their support. For instance, during photocatalytic processes over $\mathrm{Pt} / \mathrm{TiO}_{2}$, electron-hole pairs are generated in $\mathrm{TiO}_{2}$ upon band-gap irradiation. Subsequently, during reduction reactions, the photo-generated electrons in the conduction band of $\mathrm{TiO}_{2}$ migrate to the $\mathrm{Pt}$ NPs, while the holes remain in the valence band during the oxidation reaction [503]. An epitaxial relationship between the Pt NPs and the $\mathrm{TiO}_{2}$ support has been shown to facilitate the transfer of charge between the NPs and the semiconducting support, and therefore, to promote the near-UV absorption and enhance the photocatalytic activity [503-505].

Although it was mentioned in the previous section that the SMSI-induced NP encapsulation usually hinders the catalytic activity of the NPs, in some cases (e.g. iron oxide-supported Pt and Pd NPs) such encapsulation may in fact increase the activity [290; 506-512]. Figure 42(a) shows STM images of the encapsulating layer formed on the top facet of flat Pt NPs [i.e. Pt(111)] supported on $\mathrm{Fe}_{3} \mathrm{O}_{4}(111)$ after annealing at $850 \mathrm{~K}$, with the enlarged inset showing atomic resolution. This structure is identical to $\mathrm{FeO}(111)$ grown on $\mathrm{Pt}(111)$ [513; 514]. As expected, the $\mathrm{CO}$ uptake from encapsulated Pt NPs drops significantly as compared to that on NPs annealed at temperatures below the encapsulation onset $[10 ; 253]$. Therefore, the question remains of how the encapsulated system could show a higher $\mathrm{CO}$ oxidation activity. To compare the $\mathrm{CO}$ oxidation activity of platinum and iron oxide-coated platinum, first we should consider the reaction pathway. While the 
CO oxidation reaction on bare Pt follows the Langmuir-Hinshelwood mechanism $[515 ; 516]$, such reaction on iron oxide most likely follows a Mars-van Krevelen pathway [506; 510]. However, the $\mathrm{FeO}$ layer itself does not seem to be very active for the oxidation of $\mathrm{CO}$ either. To understand this phenomenon, it should be kept in mind that the system under study may undergo drastic changes when the UHV conditions are switched to more practical high pressure conditions (e.g. mbar pressure), and sometimes what is learned in UHV may not be applicable to the realistic reaction conditions. Figure 42(b) shows an STM image of the encapsulated $\mathrm{Pt} / \mathrm{Fe}_{3} \mathrm{O}_{4}$ system after it is exposed to 20 mbar of oxygen, demonstrating a clear surface restructuring. Figure 42(c) shows the schematic of the $\mathrm{FeO}(111)$ layer that is transformed to a O-Fe-O layer upon exposure to oxygen in the mbar range $[508 ; 510]$. Since the binding of the top oxygen layer on O-Fe-O is much weaker than that of oxygen on the $\mathrm{FeO}$ structure, a lower barrier is obtained for $\mathrm{CO}$ oxidation on the trilayer iron oxide [510]. Therefore, the superior activity of the encapsulated system, Figure 42(d), as compared to the clean Pt system is likely due to both, a shift in the reaction mechanism (LH to $\mathrm{MVK})$, as well as to the restructuring of the iron oxide to $\mathrm{FeO}_{\mathrm{x}}(\mathrm{x}=1.8-1.9)$ under high oxygen pressure (e.g. >20 mbar) [510]. Furthermore, lean oxygen reaction conditions may result in dewetting of the iron oxide to form isolated islands that would reduce the oxide activity [508; 509; 511]. In this case, the reaction likely occurs at the oxide island-Pt metal perimeter sites [508; 509; $511]$. 

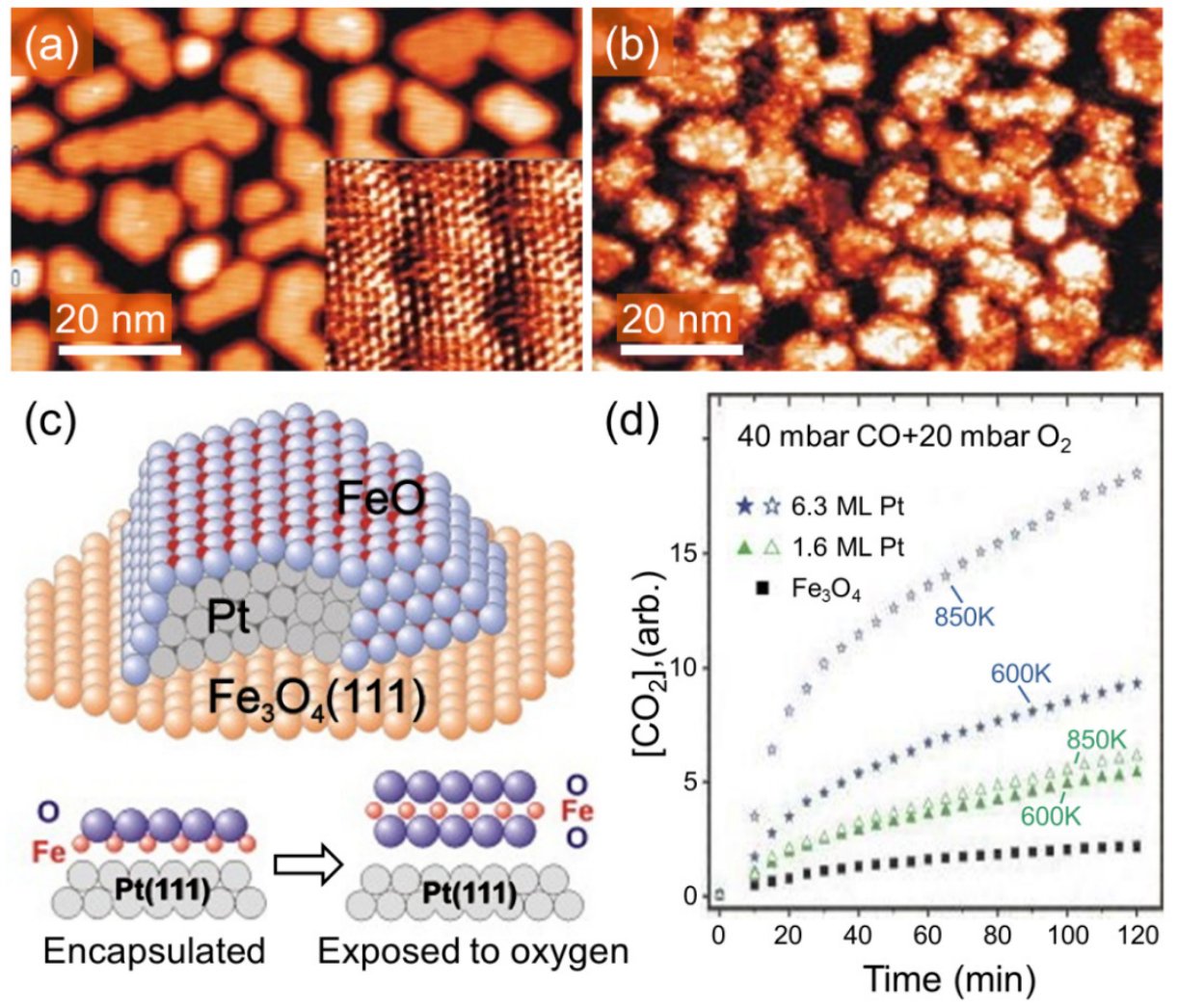

Figure 42: (a,b) STM images of Pt NPs (2.3 ML coverage) supported on $\mathrm{Fe}_{3} \mathrm{O}_{4}(111)$ films annealed in vacuum at $850 \mathrm{~K}$. The image in (b) was acquired after exposure to $20 \mathrm{mbar}_{2}$ at $450 \mathrm{~K}$ for $10 \mathrm{~min}$. The inset in (a) shows an atomically resolved image of the Pt top facets (presented in the current mode to increase the contrast). (c) Schematic of Pt NPs encapsulated by a $\mathrm{FeO}(111)$ layer. The cross-section on the bottom left shows the encapsulating $\mathrm{FeO}(111)$ film on $\mathrm{Pt}(111)$ and the one on the right the $\mathrm{O}-\mathrm{Fe}-\mathrm{O}$ film that is formed by $\mathrm{O}_{2}$ exposure. (d) $\mathrm{CO}_{2}$ production as a function of time measured on $\mathrm{Pt} / \mathrm{Fe}_{3} \mathrm{O}_{4}(111)$ at two different $\mathrm{Pt}$ coverages and $\mathrm{CO}$ and $\mathrm{O}_{2}$ partial pressures as indicated. The catalysts were annealed at $600 \mathrm{~K}$ (solid symbols) and $850 \mathrm{~K}$ (open symbols) prior to the reaction. The results for pristine $\mathrm{Fe}_{3} \mathrm{O}_{4}(111)$ films under the same conditions are shown for comparison. Time zero corresponds to the start of heating of a catalyst to the reaction temperature $(450 \mathrm{~K})$ with a rate of $1 \mathrm{~K} / \mathrm{s}$. Adapted from Ref. [506].

\subsection{Oxidation state}

Metal oxides are widely used for many catalytic reactions which in many cases include nonnoble metals such as vanadium, molybdenum and iron [517-523]. In most catalysts the particle size plays an important role in the stability of surface oxides on clusters, and this can in turn affect catalytic activity of the NPs if the oxides become involved in the reaction (e.g. through Mars-van Krevelen processes [524]), or if they are detrimental (e.g. if they block otherwise active reaction sites). 
It is known from thermodynamics that the transition from bulk oxide to bulk metal is rather sharp, and therefore, for any given temperature, there is only an $\mathrm{O}_{2}$ pressure (i.e. dissociation pressure) in which the oxide and metallic species could coexist [525]. However, in the case of extended surfaces, adding other thermodynamic considerations such as the surface energies of the metal, the oxide and the oxide-metal interface allow the formation of thin oxide layers in oxygen pressures much lower than the dissociation pressure, depending on specific thermodynamic properties of the oxide $[112 ; 525-527]$. In addition, the structure of the thin oxide layers formed on the metal surface may be completely different than that of the bulk oxide [528]. It is not surprising that the complexity associated with oxide formation increases in case of NPs, where other thermodynamic considerations should be included (e.g. the presence of low-coordinated atoms, strain, modified electronic structure, etc.) [529; 530].

In general, small NPs are better at dissociating oxygen and the size of the NPs has a direct effect on the onset conditions for oxide formation and its stability, with small NPs forming oxides easier with stronger oxygen binding. For instance, using TPD and XPS, oxide species were more easily stabilized on small Au NPs, and the desorption energy was shown to be higher for smaller NPs [71; 252; 531]. Figure 43 shows an example of similar size-dependent oxide formation observed by XPS on micelle-prepared Pt NPs with average size of $2.0 \mathrm{~nm}$ and $3.6 \mathrm{~nm}$. Upon atomic oxygen exposures $\left(\mathrm{O}_{2}\right.$-plasma) at room temperature the formation oxide species (e.g. $\mathrm{PtO}_{2}$ and $\mathrm{PtO}$ ) was observed. Figure 43(a,c) shows the phase content of the distinct Pt species as a function of the atomic oxygen exposure (in Langmuir units). Using identical atomic oxygen exposures, decreasing the NP size was found to favor their ability to form oxides. In both samples, $\mathrm{Pt}^{0}$ is converted to $\mathrm{PtO}$ and $\mathrm{PtO}_{2}$, although the oxidation of $\mathrm{Pt}$ appears to be more favorable for the smaller NPs $(2.0 \mathrm{~nm})$, since the maximum content of $\mathrm{PtO}_{\mathrm{x}}$ species (nearly complete oxidation) was achieved for a lower total atomic oxygen exposure $(\sim 40 \mathrm{~kL}$ for $2.0 \mathrm{~nm}$ NPs versus $\sim 110 \mathrm{~kL}$ for $3.6 \mathrm{~nm}$ NPs), Figure 43(a,c). In addition, the decomposition of oxide species upon annealing in UHV was studied using XPS, and it was found that in all samples, a two-step thermal decomposition process occurs: $\mathrm{PtO}_{2} \rightarrow \mathrm{PtO} \rightarrow \mathrm{Pt}$ [112]. In contrast to the case of $\mathrm{Pt}(111)$, where no oxides were detected above $700 \mathrm{~K}, 10-20 \% \mathrm{PtO}$ was detected on the NP samples (2-6 nm range) at the same temperature [112]. 

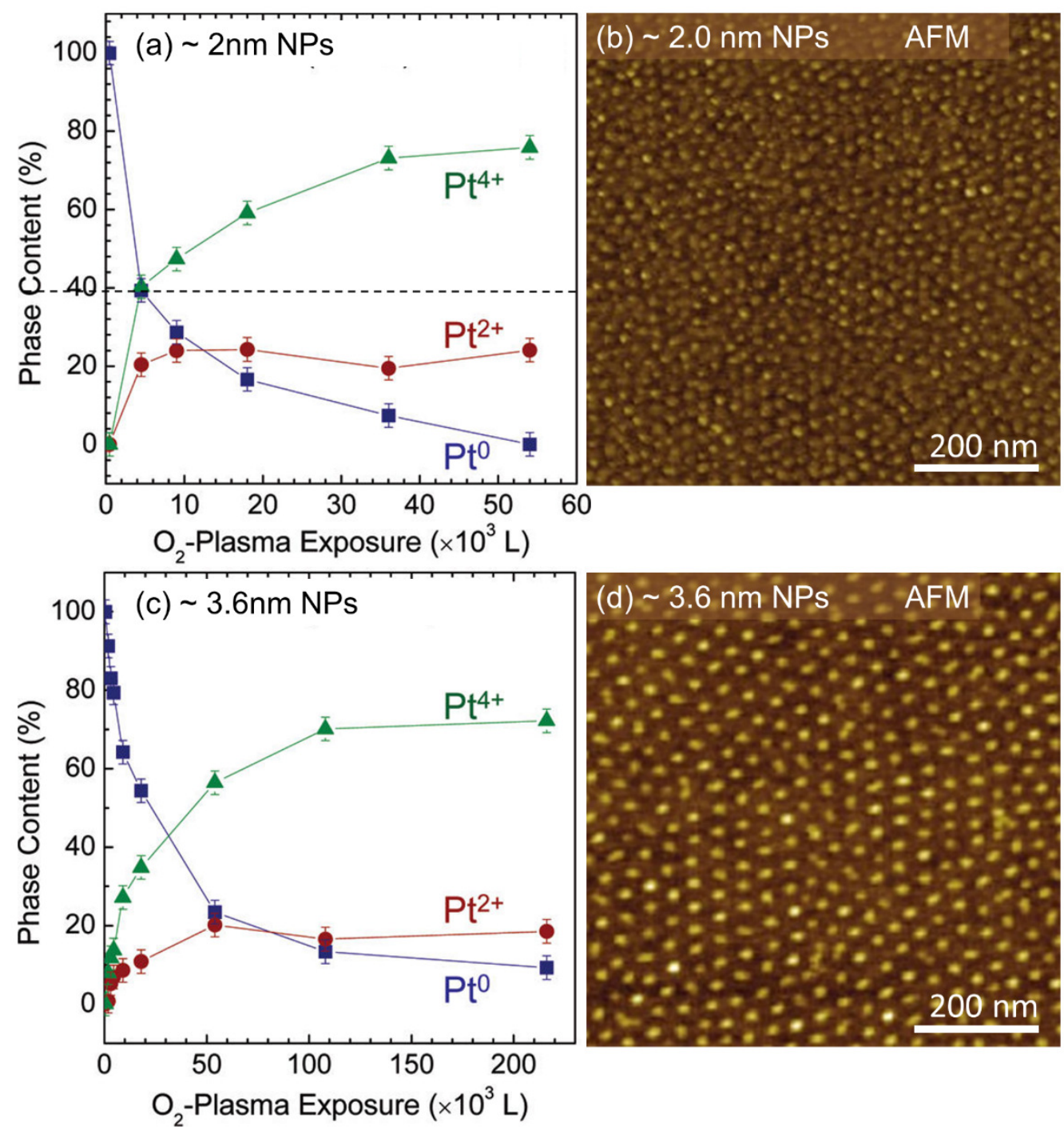

Figure 43: Phase content (\%) of Pt oxide species obtained from the analysis of the XPS spectra obtained upon room temperature atomic oxygen exposure: (a) $\sim 2 \mathrm{~nm}$, and (c) $\sim 3.6 \mathrm{mn}$ Pt NPs. (b,d) AFM images of $\mathrm{Pt} / \mathrm{SiO}_{2} / \mathrm{Si}(001)$ after removal of the encapsulating polymers, (b) $\mathrm{S} 1$, and (d) S2. adapted from Ref. [112]

\section{(a) Adsorption properties}

In general, the adsorbate binding energy is lower on the metal oxides than that on the corresponding metals [516; 532]. For example, Figure 44 shows the oxygen chemisorption energy $\left(\mathrm{E}_{0}\right)$ for different $4 \mathrm{~d}$ and $5 \mathrm{~d}$ metals and their corresponding metal oxides [532]. It could be clearly seen that the oxides have lower chemisorption energy. Depending on the binding energy, the adsorption could occur following a molecular (Region I) or atomic (Region II) mechanism. However, such reduced binding energy could be actually helpful when it comes to certain catalytic 
reactions. As it was discussed in section 3 (see Figure 25), too weak or too strong adsorbate binding energy would hinder the catalytic activity, and therefore the oxide formation could increase the activity by shifting the adsorbate binding energy into the optimum region. In the next section, the role of surface oxidation on catalytic activity will be discussed in more detail.

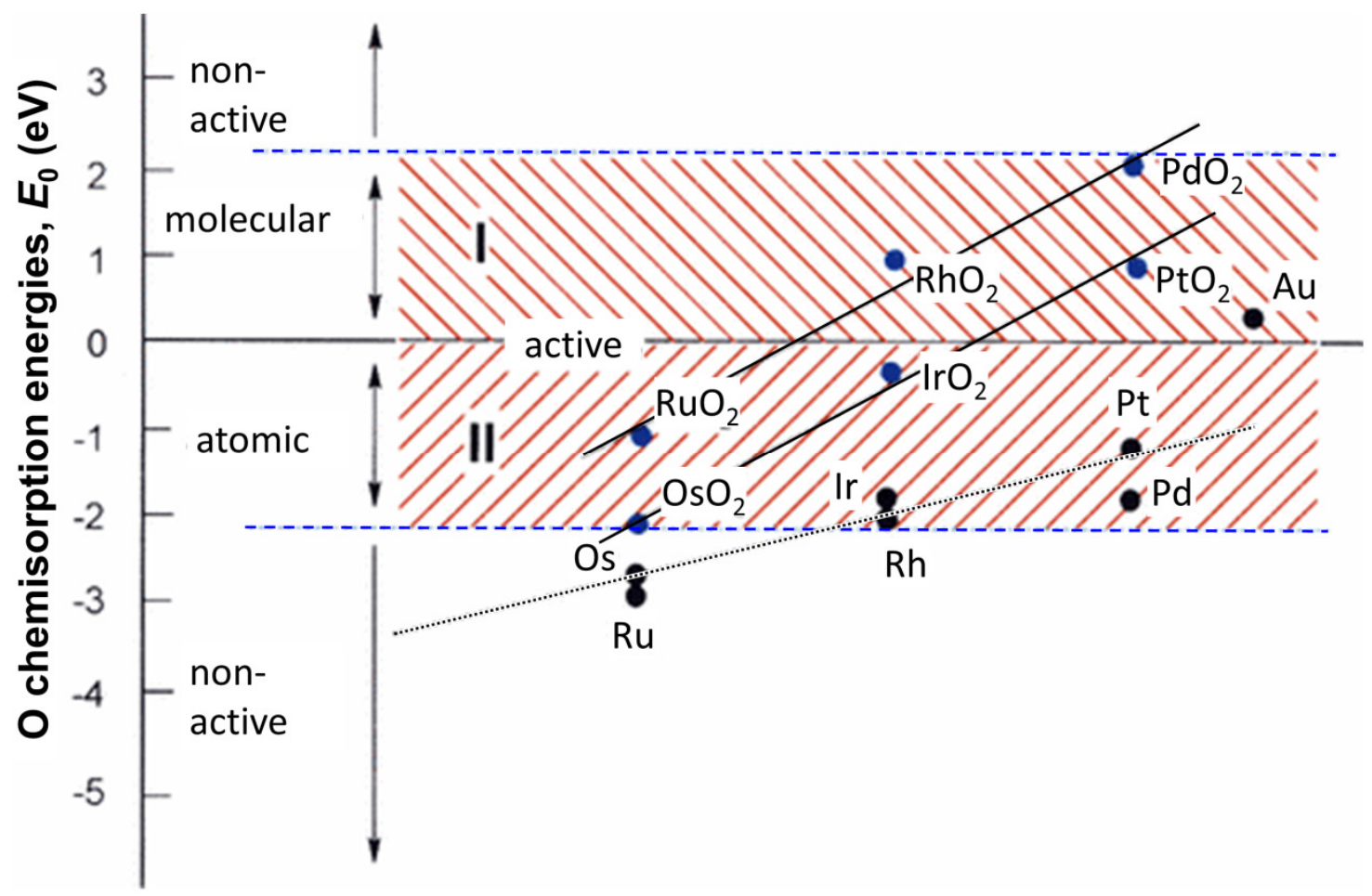

Figure 44: Calculated $\mathrm{O}$ chemisorption energies, $\mathrm{E}_{0}$ with respect to $\mathrm{O}_{2}$ in the gas phase on some $4 \mathrm{~d}$ and $5 \mathrm{~d}$ metals and metal oxides. The $\mathrm{O}$ is on the fcc hollow site on $\operatorname{Ir}(111), \operatorname{Rh}(111), \operatorname{Pt}(111)$, and $\operatorname{Pd}(111)$, and on the hep hollow site on $\mathrm{Os}(0001)$ and $\mathrm{Ru}(0001)$. The $\mathrm{O}$ atoms on the $\mathrm{MO}_{2}(110)$ surfaces $(\mathrm{M}=\mathrm{Ru}, \mathrm{Os}, \mathrm{Rh}, \mathrm{Ir}, \mathrm{Pd}$, and $\mathrm{Pt})$ are on the coordinatively unsaturated sites. Adapted from Ref. [532].

\section{(b) Effect on reactivity}

The formation of oxide species might be beneficial for the catalytic activity in certain reactions, while in others it may hinder it [533]. In general, metal oxides appear to be more reactive than the metallic state for the $\mathrm{CO}$ oxidation reaction [516]. An intriguing example is the case of CO oxidation on $\mathrm{Ru}$ [534; 535];Peden, 1986 \#423[536]. It was found that the CO oxidation rate on metallic $\mathrm{Ru}$ is an order of magnitude less than that on other noble metals such as $\mathrm{Pt}, \mathrm{Pd}$ and $\mathrm{Rh}$ under low pressure conditions [535]. However, under higher pressure of oxygen this trend is reversed, and Ru has a better performance than other noble metals [537]. It was first suggested that 
the monolayer of chemisorbed oxygen that forms on the $\mathrm{Ru}(0001)$ surface is responsible for the high activity of $\mathrm{Ru}$ under high oxygen pressure [537]. A DFT study similarly proposed the possibility of the one monolayer coverage of chemisorbed oxygen $(\theta=1)$, where the lower binding energy of oxygen to the surface (as compared to that for $\theta<1$ ) explained the higher activity of Ru under high oxygen pressure conditions [538; 539]. However, later on it was described that the CO reaction rate on the $\mathrm{Ru}(0001)$ surface covered with less than $3 \mathrm{ML}$ of oxygen (oxygen in excess of $1 \mathrm{ML}$ being located subsurface) is very low, while it increases two orders of magnitude for higher coverages [540]. The Eley-Rideal mechanism was suggested for this reaction, with gas phase CO reacting with surface chemisorbed oxygen. Therefore, the higher reaction rate for higher oxygen coverage was explained based on the repulsive oxygen-oxygen interaction and the destabilization of surface oxygen at the onset of oxide formation [540]. After it was accepted that the active species for $\mathrm{CO}$ oxidation was $\mathrm{RuO}_{2}$, a new debate started on the reaction mechanism. DFT calculations suggested an origin based on the Mars-van Krevelen (MVK) mechanism [524], in which the $\mathrm{CO}$ binds to $\mathrm{Ru}$ atoms in between the oxygen rows and then it reacts with oxygen in the $\mathrm{RuO}_{2}$ lattice [541]. The consumed oxygen will later on be substituted by oxygen from $\mathrm{O}_{2}$ dissociation [541]. However, later studies suggested that the reaction mechanism follows the Langmuir-Hinshelwood pathway instead of MVK. In this model, both CO and $\mathrm{O}$ are adsorbed species, and the oxygen from the oxide structure does not play a direct role in the reaction [542]. TPD experiments using isotopically labeled oxygen showed that both the adsorbed oxygen (on top) and the bridging oxygen are equally involved in the oxidation of $\mathrm{CO}$, although the on top oxygen has a lower binding energy than that on bridging atoms [536]. Similarly, in the case of $\mathrm{NH}_{3}$ oxidation on $\mathrm{RuO}_{2}(110)$, the main reactions occur mostly along the linear chain of undercoordinated $\mathrm{Ru}$ atoms, but with a significant contribution from bridge $\mathrm{O}$ atoms [543].

The effect of oxide species is even more complex in the case of Ru NPs. Using in situ DRIFT and temperature programmed reduction (TPR) measurements it was suggested that the best catalytic performance is achieved when a core-shell structure is realized comprising a metallic core covered with a thin oxide layer [544]. By changing the ratio of $\mathrm{CO}$ to $\mathrm{O}_{2}$ a deactivation process was observed, originating from either the partial reduction of the $\mathrm{Ru}$ oxide, or the complete oxidation of the NPs. Figure 45 shows the schematic of the catalytic deactivation of supported Ru NPs. Both, metallic Ru and pure $\mathrm{RuO}_{2}$ NPs were found to have lower activity than the core-shell structures [544-548]. The $\mathrm{RuO}_{2}$ particles expose preferentially (110) and (100) 
facets. Upon $\mathrm{CO}$ oxidation under net oxidizing conditions, the active $\mathrm{RuO}_{2}(100)$ facets of the $\mathrm{RuO}_{2}$ NPs transform into the inactive $\mathrm{RuO}_{2}(100)-\mathrm{c}(2 \times 2)$ phase. On the other hand, although $\mathrm{RuO}_{2}(110)$ facets are catalytically active, the $\mathrm{RuO}_{2}$ NPs expose predominantly catalytically inactive $\mathrm{RuO}_{2}(100)-\mathrm{c}(2 \times 2)$ facets. In addition, part of the $\mathrm{RuO}_{2}(110)$ facets will deactivate also under oxidizing conditions due to their roughening, thereby forming additional $\mathrm{RuO}_{2}(100)$ facets, which reconstruct into the inactive $\mathrm{c}(2 \times 2)$ phase. The ultrathin $\mathrm{RuO}_{2}$ layer supported on a metallic $\mathrm{Ru}$ core which is the most active state could be regenerated by complete reduction of the $\mathrm{RuO}_{2}$ particle followed by a mild re-oxidation below $500 \mathrm{~K}$ [544; 549].

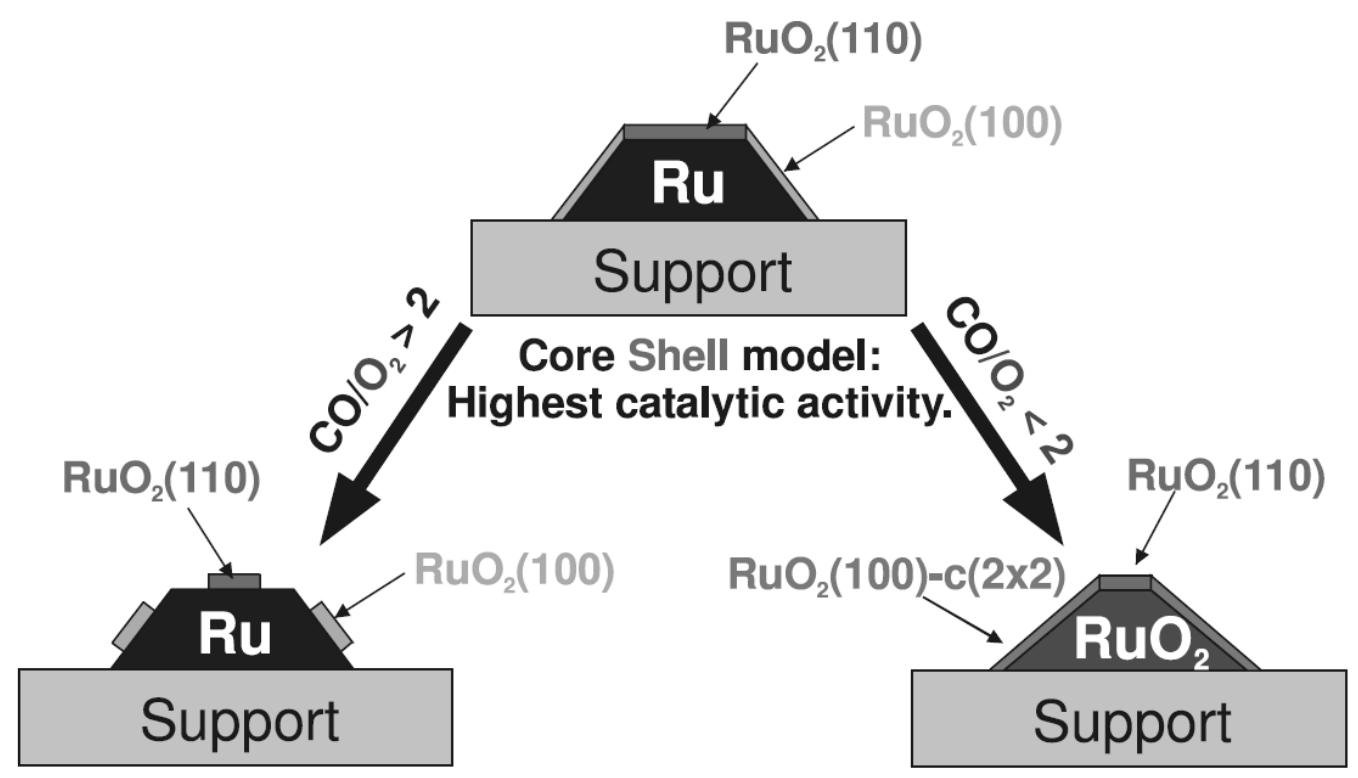

Figure 45: Structural deactivation of supported $\mathrm{RuO}_{2}$ catalysts during $\mathrm{CO}$ oxidation under reducing and oxidizing reaction conditions. Under reducing reaction conditions the active $\mathrm{RuO}_{2}$ $\mathrm{NP}$ becomes chemically reduced, exposing inactive metallic $\mathrm{Ru}$ patches. Under oxidizing reaction conditions the Ru NP is fully oxidized and exposes inactive $\mathrm{c}(2 \times 2)$ areas. Adapted from Ref. [544].

A size-dependent study of CO oxidation over Ru NPs showed that large NPs $(6 \mathrm{~nm})$ have 8 times higher activity than small $2 \mathrm{~nm}$ NPs, which was explained based on the complete oxidation of the small NPs in contrast to the stabilized core-shell structure in the larger NPs [315]. This example clearly shows the complexity of this subject and the long scientific effort that will still be 
required in the years to come in order to shed light into some elusive cases such as the one described above regarding the nature of the active reaction species and reaction mechanism.

Different oxide structures have been shown to exist on extended Pt and Pd surfaces during CO oxidation, depending on the specific reaction conditions, including the pressure and the ratio of $\mathrm{O}_{2} / \mathrm{CO}[15 ; 550-553]$. Such different oxide structures were found to affect the catalytic activity of the NPs, but regardless of the oxide structure, the platinum oxide species are found to increase the CO oxidation activity $[15 ; 550-552]$.

It has been reported that the oxidation of $\mathrm{CO}$ on metallic $\mathrm{Pt}$ and $\mathrm{Pd}$ surfaces occurs via a Langmuir-Hinshelwood mechanism involving the adsorption of both $\mathrm{CO}$ and oxygen on the surface. In this example, the low CO oxidation activity of the metallic surface is explained based on $\mathrm{CO}$ poisoning [550]. On the other hand, the adsorption of $\mathrm{CO}$ is significantly weaker on the oxidized surface and the reaction mechanism shifts to an Eley-Rideal process in which the gas phase $\mathrm{CO}$ reacts with lattice oxygen in the oxide [553]. According to this finding, the oxidation state of Pt and Pd catalysts during the $\mathrm{CO}$ oxidation reaction may vary drastically depending on the $\mathrm{CO} / \mathrm{O}_{2}$ ratio as well as the reaction temperature [550].

Furthermore, under certain conditions, an isothermal self-sustained oscillatory behavior was observed even without any variation in the gas feed composition, Figure 46. Examples of this phenomenon can be found for several reactions [554-557], including the partial oxidation of methane on $\mathrm{Pd}$ catalysts [554], $\mathrm{N}_{2} \mathrm{O}$ decomposition on $\mathrm{Cu}[558 ; 559]$, and the oxidation $\mathrm{CO}$ oxidation on $\mathrm{Pd}[560 ; 561]$ and $\mathrm{Pt}[551 ; 562-564]$. Although such oscillatory behavior is known since a number of years, its origin has been the subject of debate for a long time [515; 565]. Under low pressure conditions (e.g. $<10^{-3}$ Torr), the oscillatory behavior is explained based on variations in the surface structure of the active catalyst, in particular, to different reconstructions (e.g. $\operatorname{Pt}(100)$ [566] and $\mathrm{Pt}(110)$ [567]). In this case, no oxide formation is observed, and the oscillations appear to be the result of a shift in reaction kinetics from a low activity LH branch (CO dominated) to a high activity LH branch (O dominated) [566; 567].

Nevertheless, a different explanation is given to the oscillations observed at high pressure (e.g. atmospheric pressure) in reactions such as $\mathrm{CO}$ oxidation, namely, the restructuring of the surface due to the formation of surface oxides and their subsequent decomposition $[562 ; 564 ; 565$; $568 ; 569]$. Figure 46 shows the direct correlation between the appearance of a rough and oxidized surface with the high $\mathrm{CO}$ to $\mathrm{CO}_{2}$ conversion rate. Under these particular reaction conditions, the 
oscillation in the reaction rates occur due to oscillations in the surface structure. The high activity of $\mathrm{CO}$ oxidation on the Pd oxide surface results in the consumption of oxygen and reduction of the surface to Pd metallic. However, the lower activity of the metallic surface allows the excess oxygen to form Pd oxide, which in turn will increase the reaction rate again [565]. Similar reaction rate oscillations have also been reported for $\mathrm{CO}$ oxidation on $\mathrm{Pt}$ [15; 550; 551].
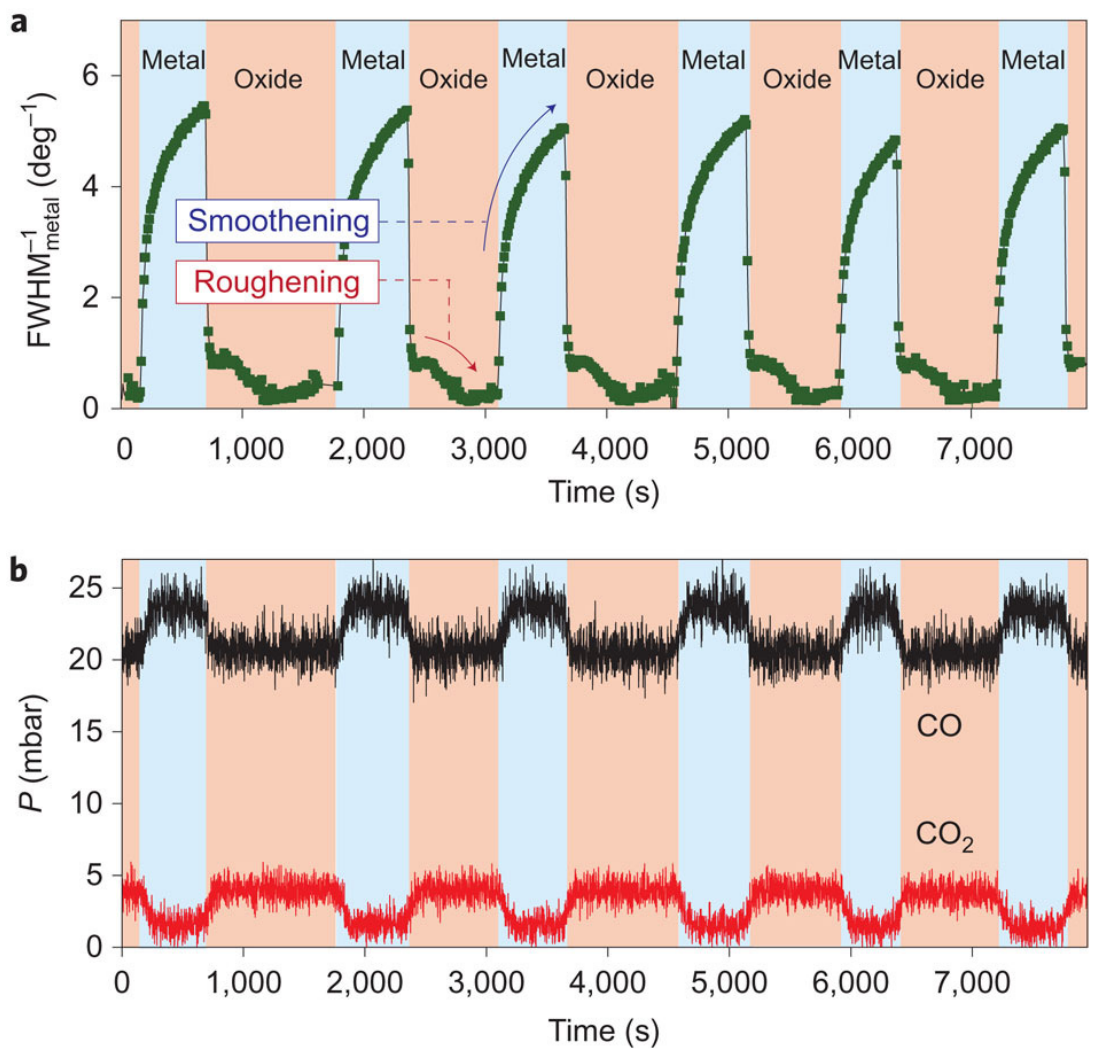

Figure 46: Spontaneous oscillations in the $\mathrm{CO}$ oxidation rate on $\mathrm{Pd}(001)$ measured by surface $\mathrm{X}$ ray diffraction (SXRD) and mass spectrometry. The measurements were performed at a temperature of $447 \mathrm{~K}$ in a constant flow of a mixture of $\mathrm{CO} / \mathrm{O}_{2} / \mathrm{Ar}$ gas with an oxygen pressure of $500 \mathrm{mbar}$, an argon pressure of $675 \mathrm{mbar}$, and a CO pressure of $25 \mathrm{mbar}$ flowing at $50 \mathrm{ml}_{\mathrm{n}} \mathrm{min}^{-1}$. (a) $\mathrm{FHWM}_{\text {metal }}{ }^{-1}$ of the diffraction peak at $(h, k, l)=(1,0,2)$. This parameter is inversely proportional to the step density and a measure of the smoothness of the surface. (b) Partial $\mathrm{CO}$ and $\mathrm{CO}_{2}$ pressures as measured by mass spectrometry. At high $\mathrm{CO}$ partial pressures $P_{\mathrm{CO}}$, the diffraction pattern shows that the surface structure is close to a bulk-like truncation of the Pd crystal. Under the former conditions, the reaction kinetics indicates that the metal surface is covered by a mixture of $\mathrm{O}$ atoms and $\mathrm{CO}$ molecules. At low $P_{\mathrm{CO}}$, new diffraction peaks are found that correspond to a thin $\mathrm{PdO}(001)$ layer. The colors indicate whether the SXRD intensities identify a metallic $\mathrm{Pd}(001)$ surface structure (light blue) or a surface covered by a thin oxide film (light red). Source: adapted from Ref. [565]. 
However, the effect of Pt oxide species in the reaction rate may vary for different reactions. For instance, while Pd oxide is better than Pd metallic for oxidation of volatile organic compounds (VOC), it may perform worse when it comes to VOC decomposition reactions [552].

Also, in clear contrast with the $\mathrm{CO}$ oxidation process, the presence of oxide species appears to hinder the activity of catalysts in NO oxidation reactions [316; 372; 570-572]. Figure 47(a) shows the $\mathrm{NO}$ oxidation conversion for three $\mathrm{Pt} / \mathrm{Al}_{2} \mathrm{O}_{3}$ catalysts with different pretreatments. The fresh samples and the one pretreated in $\mathrm{O}_{2}$ at $440^{\circ} \mathrm{C}$ show similar activity, while the sample pretreated in $\mathrm{NO}_{2}+\mathrm{O}_{2}$ at $250^{\circ} \mathrm{C}$ has the lowest conversion. This is due to the strong oxidizing effect of $\mathrm{NO}_{2}$ and demonstrates that for NO oxidation reactions, the formation of Pt oxides is not favorable [573].

In addition, the hysteresis phenomena observed for the NO oxidation reaction is the inverse of that observed for CO oxidation [573; 574]. Figure 47(b) shows the inverse hysteresis in NO oxidation conversion with the heat up ramp showing higher activity than the cooling down cycle. This behavior is explained based on a reversible oxidation of the platinum surface by $\mathrm{NO}_{2}$ at high temperature and a reduction of the platinum surface by NO at lower temperatures [574]. As expected, if a step-wise temperature ramp (in which the temperature is held steady for 30 min at selected temperatures) is applied instead of the continuous ramp, the hysteresis effect decreases significantly. The hysteresis effect is due to the fact that the system did not have enough time to reach its equilibrium state and therefore, by increasing the dwell time or decreasing the heatingcooling rate, both, the heat up and cool down cycles will approach a thermodynamic equilibrium level. 

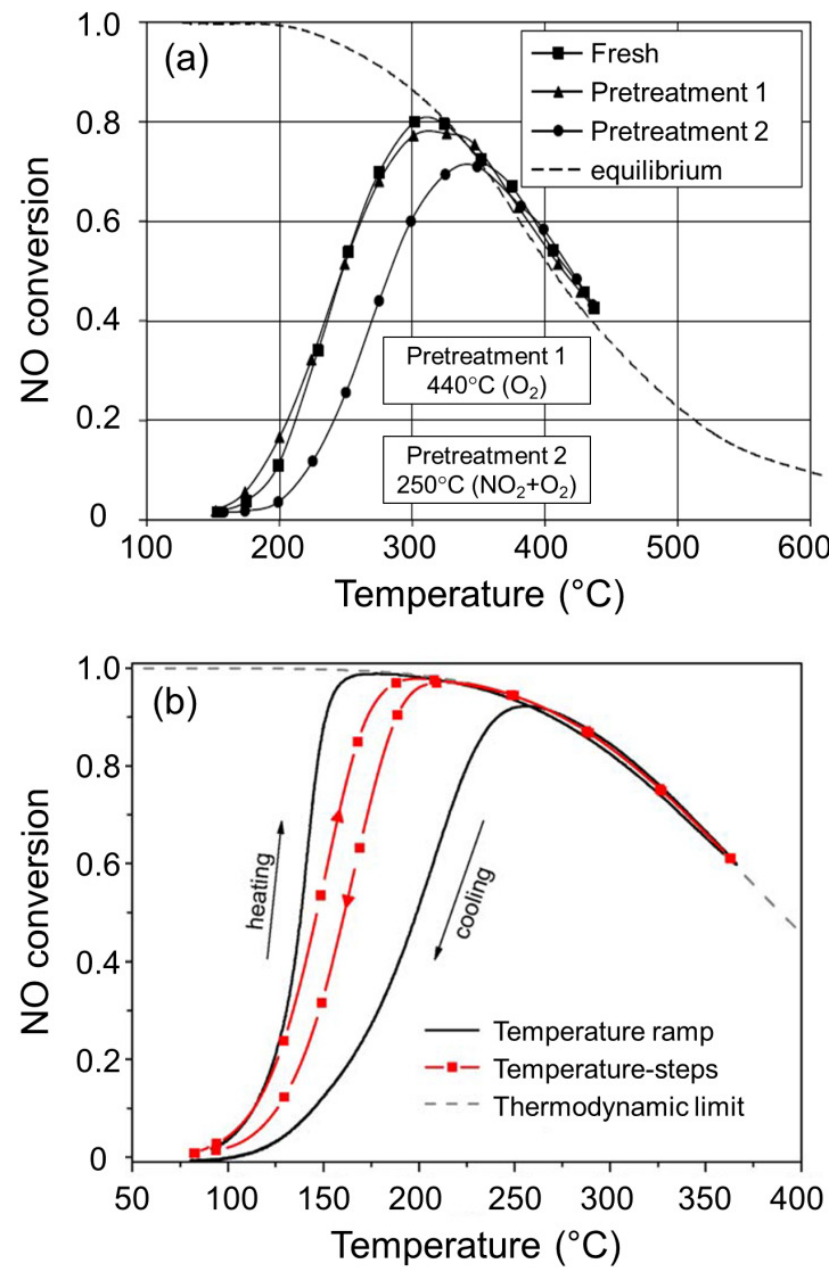

Figure 47: (a) Oxidation of $\mathrm{NO}$ over reduced and pretreated $\mathrm{P} / / \mathrm{SiO}_{2}$ catalyst (5\% loading). Feed: $500 \mathrm{ppm} \mathrm{NO}, 10 \% \mathrm{O}_{2}, 5 \% \mathrm{H}_{2} \mathrm{O}$, balanced by $\mathrm{N}_{2}$. Pretreatment $1: 10 \%$ oxygen, $5 \% \mathrm{H}_{2} \mathrm{O}$ in $\mathrm{N}_{2}$ at $440^{\circ} \mathrm{C}$ for $2 \mathrm{~h}$. Pretreatment 2, $500 \mathrm{ppm} \mathrm{NO}, 10 \%$ oxygen, $5 \% \mathrm{H}_{2} \mathrm{O}$ in $\mathrm{N}_{2}$ at $250^{\circ} \mathrm{C}$ for $2 \mathrm{~h}$ [575]. (b) $\mathrm{NO}$ oxidation reaction on $\mathrm{Pt} / \mathrm{Al}_{2} \mathrm{O}_{3}$ catalysts: the conversion is shown for the first run of a light-off/light-down-experiment (black) and the first run of a temperature-step-experiment (symbols). During the light-off/light-down-experiments the temperature was increased from 80 to $370^{\circ} \mathrm{C}$ with a heating rate $(\beta)$ of $5^{\circ} \mathrm{C} / \mathrm{min}$ and was cooled with the same rate to $80^{\circ} \mathrm{C}$. The temperature was increased from 80 to $370^{\circ} \mathrm{C}$ in $\mathrm{N}_{2}$ before decreasing to $80^{\circ} \mathrm{C}$. At selected temperatures, the heating/cooling was paused. After reaching a constant temperature, the feed gas was switched from $\mathrm{N}_{2}$ to the standard feed composition ( 450 vol. ppm NO, 6 vol. $\% \mathrm{O}_{2}$ ) for $30 \mathrm{~min}$ [574].

Figure 48 shows the activity of $\mathrm{Pt} / \gamma-\mathrm{Al}_{2} \mathrm{O}_{3}$ catalysts for $\mathrm{NO}$ oxidation for samples with different size (Pt1-Pt3)[301]. Although the TEM diameter obtained for Pt NPs in samples Pt1-Pt3 is similar ( 0.6-0.7 nm), the EXAFS coordination numbers (NN1) show significant variations among these three samples, with Pt1 having the smallest NN1 value and Pt3 the largest. Here, it should be clarified that the difference in EXAFS coordination numbers is most likely due to 
different NP shapes in these samples due to its distinct preparation, with those with 3D NPs (e.g. Pt3) having larger NN1 values as compared to those with 2D, flat NPs (e.g. Pt1).

The normalized conversion in Figure 48(a) shows higher activity for NPs with larger coordination numbers [301]. The average $1^{\text {st }}$ nearest neighbor atomic coordination numbers are obtained from EXAFS measurements and are indicative of both the size and shape of the NPs, with lower values corresponding to smaller or flatter NPs. Figure 48(b) shows the TOFs that are obtained at low temperature $\left(<200^{\circ} \mathrm{C}\right)$, where the conversion is below $20 \%$. Interestingly, the sample with smaller NPs with lower conversion has a lower activation energy barrier. It has been shown that the rate limiting step in the $\mathrm{NO}$ oxidation reaction is the dissociation of $\mathrm{O}_{2}$ [222]. Therefore, the lower activation energy barrier for smaller NPs in sample Pt1 demonstrates the higher binding energy and lower dissociation energy of $\mathrm{O}_{2}$ on those NPs. However, as can be seen in Figure 48(b), the lower conversion rate obtained from smaller NPs is mainly due to a significantly lower prefactor ( 4 orders of magnitude). This is due to the compensation effect [333-335] that has been described in section 3. Using XANES and EXAFS measurements, PtO species were found in all samples, but the highest degree of oxidation was observed on samples with the smallest NPs. Since for NO oxidation Pt oxide species are less active than metallic platinum, the easier oxide formation on smaller NPs is expected to hinder their activity [316; 372; 576]. In addition, for a specific sample, reaction conditions that would favor oxide formation such as high $\mathrm{NO}_{2}$ concentration would result in the catalyst deactivation, which could be completely reversed through a reactivation process in a reducing environments [571]. 

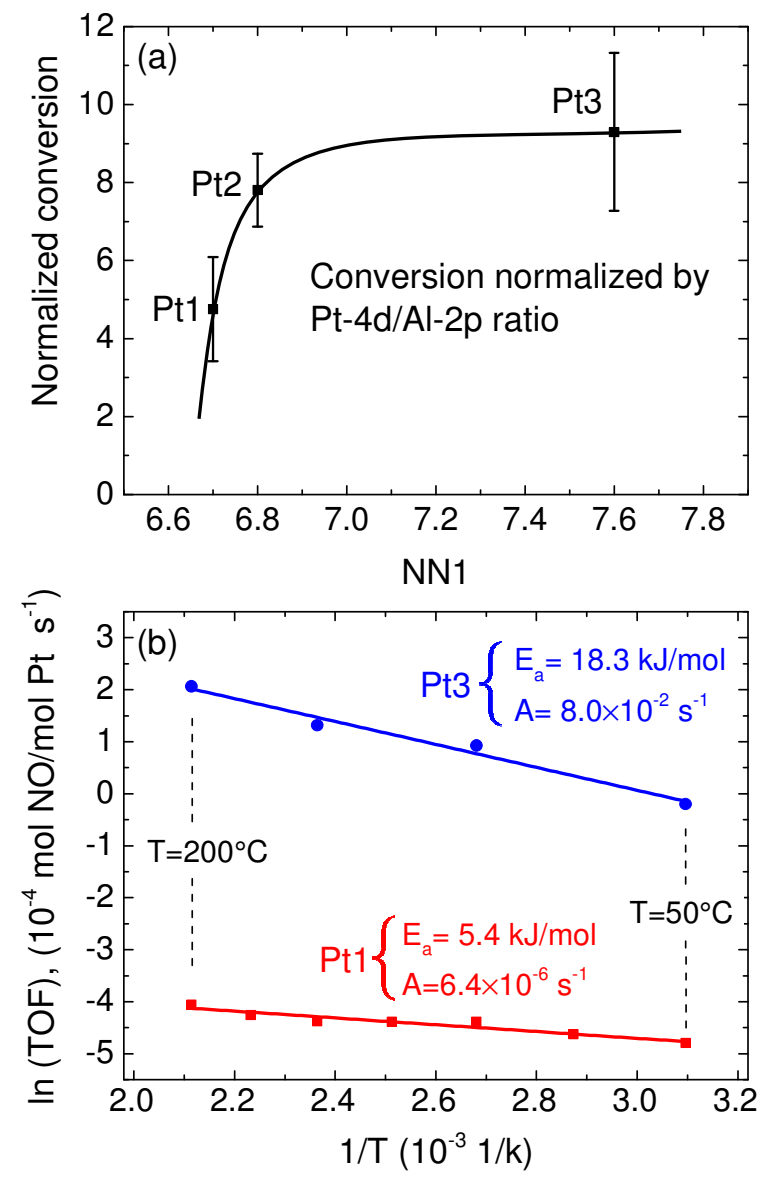

Figure 48: (a) NO conversion obtained at $150{ }^{\circ} \mathrm{C}$ normalized by the XPS data of sample Pt1 versus the $1^{\text {st }}$ nearest coordination number $(\mathrm{NN} 1)$ extracted from EXAFS measurements.

(b) Arrhenius plot of TOF obtained for Pt 1 and Pt 3 expressed as mol of NO converted per mol of surface Pt per second. The apparent activation energy and the prefactor obtained from the Arrhenius plot are also shown. Adapted from Ref. [301].

In summary, we hope that the discussion above demonstrates that the underlying origin of the distinct unusual properties observed in the catalytic reactivity of NPs as a function of the NP size is still an open question in many cases. One of the reasons for such complexity is the variety of factors that are involved concurrently, making it very challenging to systematically isolate each of those parameters. For instance, changing the NP size will not only change the number of lowcoordinated atoms on the NP surface, but also affect its electronic structure, the extent of the NPsupport interaction, as well as its shape. 


\section{Role of the nanoparticle shape on catalytic activity and selectivity}

Numerous experimental and theoretical studies have been dedicated to establishing correlations between the catalytic activity and the NP size, composition, and support [14; 101; $190 ; 214 ; 215 ; 233-235 ; 371]$. Nevertheless, the effect of NP shape seems to be more elusive and although its importance is generally accepted $[14 ; 33 ; 102-105 ; 181 ; 188 ; 209 ; 214 ; 230 ; 577-586]$, a comprehensive understanding of the thermodynamically stable shapes of NPs, how they are affected by the NP size and support, their evolution in the course of a chemical reaction, and their role in the catalytic performance is still lacking. This is due to the fact that both synthesizing sizeand shape-selected NPs and also characterizing their shape under realistic environmental conditions is still challenging. Excellent size-selection, although challenging itself, is a prerequisite to any investigation on shape effects for the deconvolution of the changes brought about by variations in the NP size.

The shape of NPs affects their performance in several ways which will be discussed in detail here. Different shapes feature different facets or special surface sites (corners, edges, kinks, etc.), affecting any surface-sensitive phenomena such as a catalytic reactions. In addition, shape can change the NP surface to bulk ratio as well as the interface with the support (eg. 3D as compared to flat 2D-like NPs). These changes could in return modify the concomitant finite size effects, NPsupport interaction, and charge transfer phenomena.

For instance, Tsung et. al. [294] reported no shape- or size-dependence for the ethylene hydrogenation rates over Pt NPs with different structures, with the reaction rates being comparable to those of Pt single crystals. In contrast, in the case of pyrrole hydrogenation, different selectivities were obtained for nanocubes as compared to nanopolyhedra.

In the next sections we divide the shape effect into two categories: (i) those which are simply due to the distinct facets featured on the NP surface, which is similar to those observed in single crystal extended surfaces, and (ii) other effects. The former effects are also occurring on very large NPs, but the latter are usually restricted to small nanosized structures.

\subsection{Effect of different surface facets}

Many catalytic reactions are known to be sensitive to the crystalline orientation of the surface. Studies on extended single crystal surfaces attributed these differences to the coordination number of surface atoms, to geometric considerations in the attachment of adsorbates, and also to the 
relative content of steps in high Miller index surfaces. Similar effects are expected to be observed on faceted NPs depending on what facets dominate the NP surface $[177 ; 181 ; 420-422 ; 587 ; 588]$.

Similarity between extended surfaces and NPs is specially expected for large NPs, where the population of atoms at corners and edges, as well as other phenomena attributed to finite size effects are negligible. For example, cubic $\mathrm{Au}$ NPs $(\sim 40 \mathrm{~nm})$ were shown to have a very high activity for the ORR in alkaline media and the reaction characteristics were found to be similar to that of $\mathrm{Au}(100)$ single crystals [589].

It has been shown that Pt NPs with cubic shape have a better activity for the oxygen reduction reaction (ORR) than those with polyhedral, truncated cubic, or spherical shape [177; 588]. Likewise, $\mathrm{Pt}(100)$ surfaces were found to be more active than $\mathrm{Pt}(111)$ for $\mathrm{ORR}$ in $\mathrm{H}_{2} \mathrm{SO}_{4}$ [420]. To understand this trend, the binding of sulfates to the Pt surface was considered. Three oxygen atoms in the sulfate structure would match better the arrangement/structure of $\mathrm{Pt}$ atoms on $\mathrm{Pt}(111)$, since they also have a three-fold symmetry as compared to $\operatorname{Pt}(100)$ with two-fold symmetry. Therefore, sulfate would easily block and poison the $\operatorname{Pt}(111)$ surface [177]. Here we have to mention that these trends are reaction and system specific, and may not be generally applicable to other similar reactions. For instance, in the case of ORR in $0.1 \mathrm{M} \mathrm{HClO}_{4}$, the $\mathrm{Pt}(111)$ surface has been found to perform better than $\mathrm{Pt}(100)$ [421], which is the opposite of the trend observed for $\mathrm{ORR}$ in $\mathrm{H}_{2} \mathrm{SO}_{4}$ over Pt [420]. Yet again, the trends observed for ORR in $0.1 \mathrm{M} \mathrm{HClO}_{4}$ for $\mathrm{Pd}$ are opposite to those of $\mathrm{Pt}$ in the same conditions [e.g. $\operatorname{Pd}(100)>\operatorname{Pd}(111)>\operatorname{Pd}(110)$ and $\operatorname{Pt}(100)<\operatorname{Pt}(111)<\operatorname{Pt}(110)][422]$.

For the catalytic oxidation of styrene using silver nanoparticles with distinct shapes, nanocubes were found to be 14 times more active than truncated triangular nanoplates, and 4 times more active than near-spherical NPs [104]. A similar trend was reported for the selective epoxidation of ethylene over $\mathrm{Ag}$ nanostructures, with pentagonal nanowires predominantly featuring $\{100\}$ facets displaying better selectivity towards ethylene oxide than spherical NPs composed mainly of $\{111\}$ facets [585; 590]. This observation was rationalized by DFT calculations on extended $\operatorname{Ag}(111)$ and $\operatorname{Ag}(100)$ surfaces [355; 585; 591]. Figure 49 shows the energy diagram for a surface oxametallacycle (OMC) intermediate converting to acetaldehyde (AC) and ethylene oxide (EO) on $\operatorname{Ag}(111)$ and $\operatorname{Ag}(100)$ surfaces. As can be seen in Figure 49(a), there is no preference for EO production on the $\operatorname{Ag}(111)$ surface, while in Figure 49(b) the lower energy barrier for EO production explains the observed $\mathrm{Ag}(100)$ selectivity toward EO. 


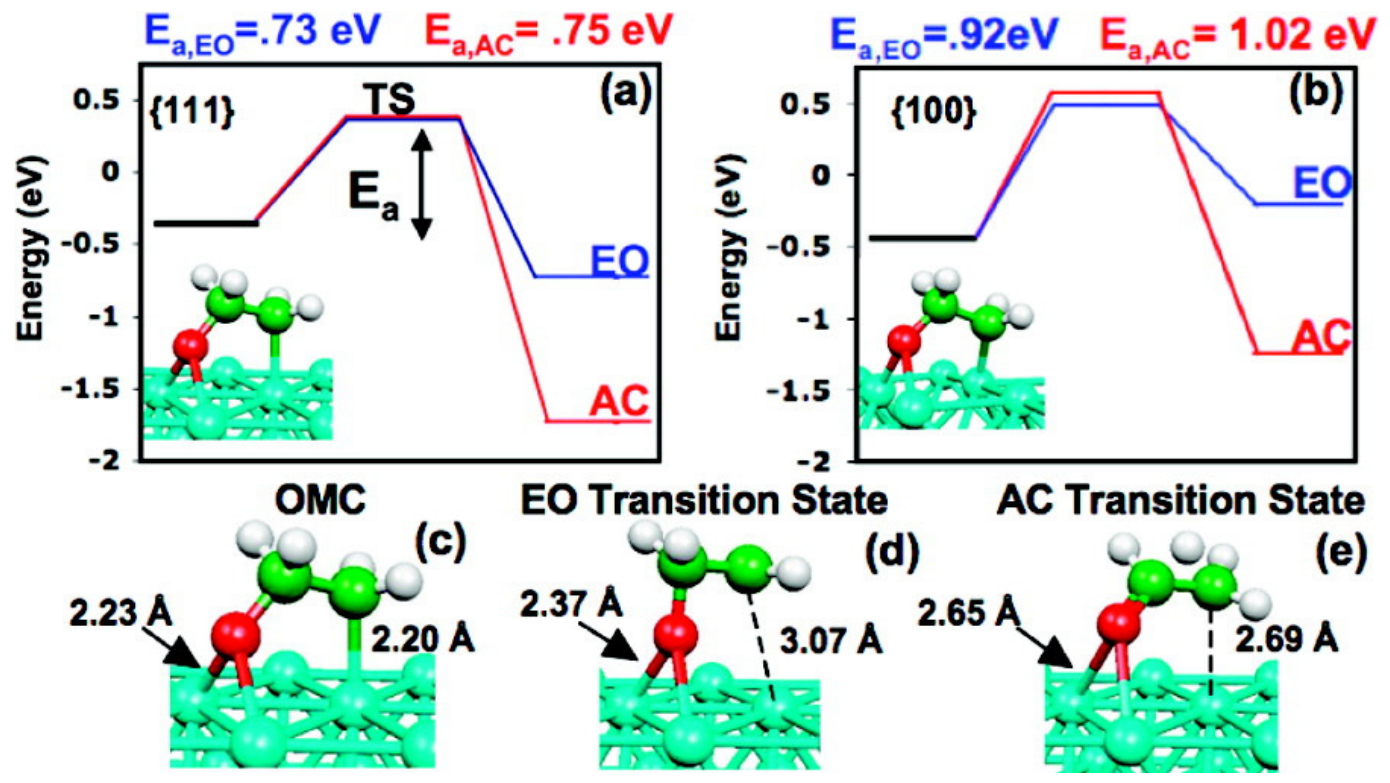

Figure 49: $(a, b)$ Potential energy surfaces for transformation of oxametallacycle (OMC) to acetaldehyde (AC) and ethylene oxide (EO) on $\operatorname{Ag}(111)$ (a) and $\operatorname{Ag}(100)$ (b). Energies are referenced to $\mathrm{O}$ (ads) and ethylene(gas). (c) OMC on $\mathrm{Ag}(111)$, (d) EO transition state on $\mathrm{Ag}(111)$, and (e) AC transition state on $\operatorname{Ag}(111)$. Adapted from Ref. [585].

In the case of oxide nanostructures, the effect of the crystalline orientation could be even more drastic than that on pure metal NPs. For instance, a shape-dependent reactivity was observed for $\mathrm{Cu}_{2} \mathrm{O}$ nanocrystals based on the facets dominating the NP surface. It was found that $\{111\}$ facets are photocatalytically active when interacting with negatively charged molecules, and $\{100\}$ facets are inert [105]. The origin of this difference could be understood based on the fact that $\mathrm{Cu}_{2} \mathrm{O}(100)$ facets contain oxygen atoms on the surface, while (111) facets feature positively charged $\mathrm{Cu}$ atoms with dangling bonds [592].

Surfaces with high Miller indices could also enhance the catalytic activity due to the high density of low-coordinated atoms at step edges. Tetrahexahedral Pt NPs featuring high-index facets such as $\{730\},\{210\},\{520\}$ have been successfully synthesized and found to have a superior performance in electro-oxidation of formic acid and ethanol as compared to Pt nanospheres [181].

As explained in section 3.2a, different facet orientations may exhibit different binding energies for adsorbates. Therefore, in a reaction involving two different reactants, significant changes in the reactivity may occur due to the resulting modified adsorbate coverages. However, on the NP surfaces the proximity of the different facets allows the diffusion of adsorbates from one facet to another. In a review by Zhdanov et al. [593] in which simulation methods for reaction 
kinetics are discussed, it is shown that such phenomena could result in reaction kinetics that are very different than those observed on extended surfaces featuring only one facet. For instance, Komanicky et.al studied the effect of the NP shape on the oxygen reduction reaction (ORR) over Pt NPs with distinct shapes prepared using electron beam lithography [33]. Based on the distinct electrocatalytic activities observed for different NP shapes, a synergistic cooperative behavior for ORR between $\{100\}$ and $\{111\}$ facets was suggested, with $\mathrm{O}_{2}$ preferentially adsorbing on (100) facets, but subsequently diffusing to $\{111\}$ facets where it is more efficiently reduced [33]. Similar behavior has also been reported for single crystal surfaces patterned with alternating (100) and (111) nanofacets, demonstrating the same proximity effect [419; 594].

The shape effect is not limited to the reaction rate, but could also be a key factor in the reaction selectivity. For example, Bratlie et al have shown shape-dependent catalytic selectivity for the benzene hydrogenation reaction using Pt NPs ( 13nm) [87]. Cubic NPs exposing $\{100\}$ facets were found to form only cyclohexane, while the cuboctahedral NPs featuring both $\{100\}$ and \{111\} facets produced both cyclohexane and cyclohexene. This finding follows the results obtained on $\mathrm{Pt}(111)$ and $\mathrm{Pt}(100)$ surfaces, but demonstrates a lower activation energy and a higher turnover frequency for NPs as compared to single crystal surfaces [87].

Higher selectivity for n-butylamine was observed for $\mathrm{Pt}$ nanocubes as compared to nanopolyhedra during the hydrogenation of pyrrole due to their more favorable ring-opening ability [294]. Again, this observation could be rationalized based on the data available on $\operatorname{Pt}(111)$ and Pt(100) single crystals [294; 595].

Furthermore, the high selectivity for the thermodynamically unfavorable isomerization of trans 2-butene to its cis counterpart observed over tetrahedral Pt NPs was assigned to the high content of $\mathrm{Pt}(111)$ facets [188]. Furthermore, sample heating resulting in changes in the NP shape and a decrease in the content of $\operatorname{Pt}(111)$ facets gave rise to a reverse selectivity trend, with cis-totrans conversion being dominant. TPD experiments on $\operatorname{Pt}(111), \operatorname{Pt}(100), \operatorname{Pt}(557)$ and $\operatorname{Pt}(110)$ single crystals were used to explain these observations showing that the trans isomers are more stable on more open surfaces such as $\mathrm{Pt}(110)$, while the cis isomers are more stable on more closed packed surfaces such as (111), (100) and (557) [188].

Enhanced reactivities and lower onset reaction temperatures were obtained during the oxidation of CO over shape-selected Pd NPs for octahedral and spherical NPs with predominantly 
$\{111\}$ facets, while an inferior catalytic performance was observed for nanocubes with $\{100\}$ facets [582].

Here, we want to highlight that in all the above cases, the different behavior of NPs with different shapes can be understood based on our knowledge from extended surfaces such as single crystals. Regardless of the much higher surface to bulk ratio of NPs as compared to single crystals, the NPs facets performance appeared to be fundamentally similar to that of the extended surfaces. In the next section, we look at other shape-dependent phenomena that may be uniquely attributed to small NPs.

\subsection{Other shape-related effects uniquely observed for NPs}

In the previous sections we established the effect of the NP size on the catalytic activity and explained it based on the presence of low-coordinated atoms, unusual size-dependent electronic properties, the NP-support interaction, as well as the oxidation state. Here we generalize all of these phenomena to be also shape-dependent. For example, having two NPs with the same size but different shape (e.g. 3D and flat 2D shapes) would affect the number of low-coordinated atoms at corner and edge sites, the number of interfacial perimeter atoms, the contact area with the support (hence the strain effect and charge transfer phenomena), and the ability to form oxides. Examples of such shape-dependent activity are provided below.

Mostafa et al. [3] studied the effect of the NP shape in the 2-propanol partial oxidation reaction using size- and shape-selected Pt NPs supported on $\gamma-\mathrm{Al}_{2} \mathrm{O}_{3}$ catalyst (Figure 50). The shape of the very small micelle-prepared Pt NPs $(0.8-1.0 \mathrm{~nm})$ was determined using a combination of TEM, STM and EXAFS analysis. A clear correlation was observed between the reaction onset temperature and the average number of missing bonds on the NP surface as a quantitative indication of the population of low-coordinated atoms. Thus, it was shown that for this reaction, having more low-coordinated atoms at corner and edge sites reduces the reaction barrier. 

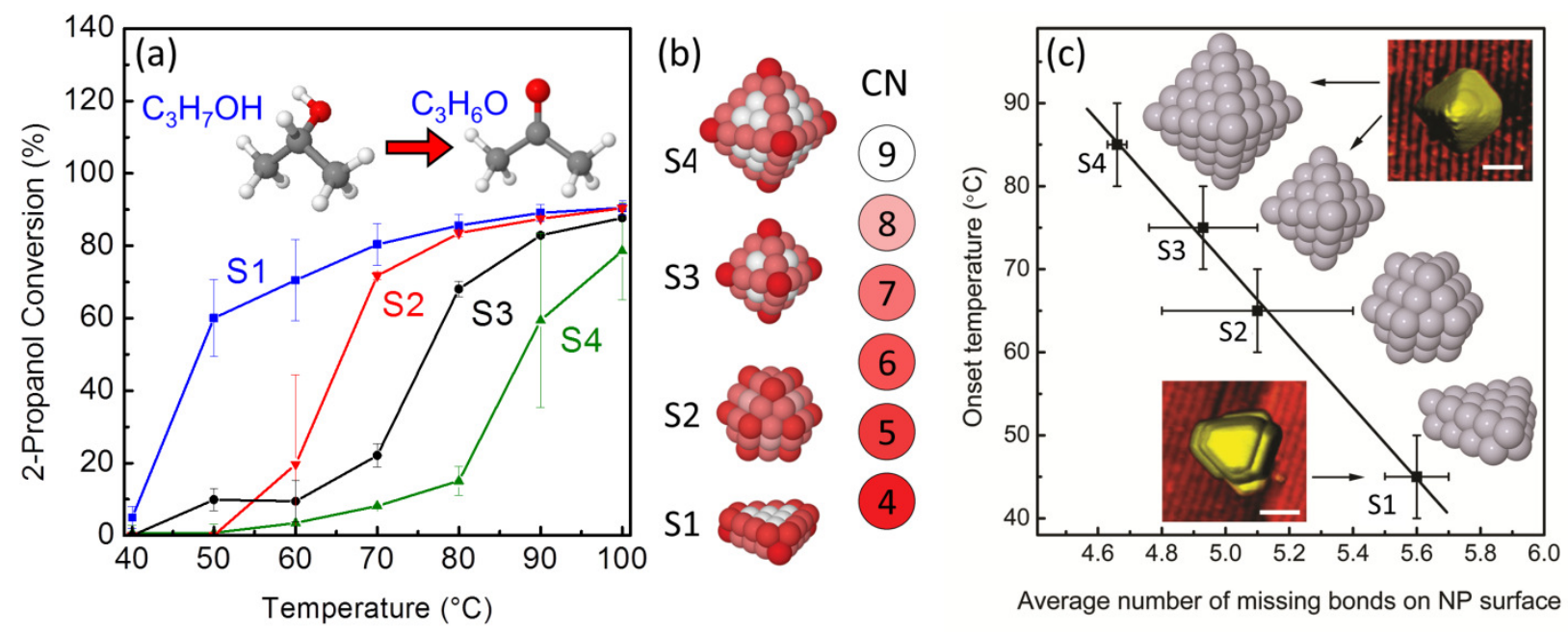

Figure 50: (a) Conversion data during the 2-propanol partial oxidation reaction to acetone over $\mathrm{Pt}$ $\mathrm{NP}(0.8-1 \mathrm{~nm}) / \gamma-\mathrm{Al}_{2} \mathrm{O}_{3}$ catalysts. (b) Schematic model shapes of samples $\mathrm{S} 1-\mathrm{S} 4$, with similar size (S1, S2, S4 $\sim \mathrm{nm}$ and S3 $\sim 0.8 \mathrm{~nm}$ ). (c) Evolution of the onset reaction temperature as a function of the number of missing bonds (related to the population of low-coordinated atoms) on the NP surface.

Narayanan et al studied the catalytic reaction between hexacyanoferrate (III) and thiosulfate ions over shape-selected Pt NPs [103]. They found that the reaction barrier is smallest on tetrahedral NPs $(4.8 \pm 0.1 \mathrm{~nm})$ and highest on cubic NPs $(7.1 \pm 0.2 \mathrm{~nm})$, with the near spherical cuboctahedron NPs $(4.9 \pm 0.1 \mathrm{~nm})$ having an intermediate activation energy. More detailed analysis of the surface composition of each catalyst revealed that the higher the population of atoms at corners and edges, the lower the activation energy.

However, not all reactions would show a higher rate on low coordinated atoms. For instance, Mistry et al. studied the catalytic oxidation of 2-butanol over size- and shape- selected Pt NPs supported on $\gamma-\mathrm{Al}_{2} \mathrm{O}_{3}$ [114]. Although the size of the NPs in different samples was found to be similar $(\sim 1 \mathrm{~nm})$, the shape of the NPs was found to be different (3D vs flat NPs). Interestingly, it was observed that the onset temperature increases with increasing number of low-coordinated atoms, namely, the reaction barrier is higher on low-coordinated atoms. This finding could be understood based on the higher stability of platinum oxide species on smaller NPs, which was detrimental for this chemical process, since the 2-butanol total oxidation reaction was found to occur more readily on metallic $\mathrm{Pt}$, as was the case also for the total oxidation of 2-propanol to $\mathrm{CO}_{2}$ [373]. 
In another study by Narayanan et al. [103] it was similarly shown that the atoms at corners and edges of the NPs are the active sites for the reaction between hexacyanoferrate (III) and thiosulfate ions (298 K-318 K temperature range). The kinetic parameters such as activation energy and average reaction rate were calculated for tetrahedral, cubic, and near spherical Pt NPs, and a correlation was found between the kinetic parameters and the fraction of atoms at corners and edges. Tetrahedral NPs with the highest number of corner and edge atoms were found to be the most reactive.

Quantum confinement phenomena are also providing a variety of opportunities in the fields of plasmonics, photocatalysis, sensing, and even nanoparticle synthesis [46; 210; 375; 596-604]. The quantum size-effect of confinement occurs when the de Broglie wavelength of the valence electrons is of the same order as the NP size itself [605]. Also, metal NPs show distinct plasmonic properties that could be used in the development of new procedures for the synthesis of nanostructures [46; 599], medical treatments [606-608], sensing applications [601; 609; 610], and plasmon-induced catalytic reactions [611-616]. The shape of the NPs has been known to affect their plasmonic properties $[210 ; 597 ; 600 ; 601 ; 617]$. For example, Figure 51 [597] shows the specific plasmonic spectral response of NPs with different shapes, with triangular nanoprisms having higher plasmon resonance wavelength than pentagonal nanoprisms, and spherical NPs showing the lowest resonance wavelength. Remarkably, the plasmonic response could be finetuned by annealing the NPs, resulting on subtle shape alterations [597]. For instance, heat treatments of triangular Ag NPs were found to make the sharp edges rounder, and as a result of this shape transformation, a red shift was observed in their plasmon resonance wavelength. 


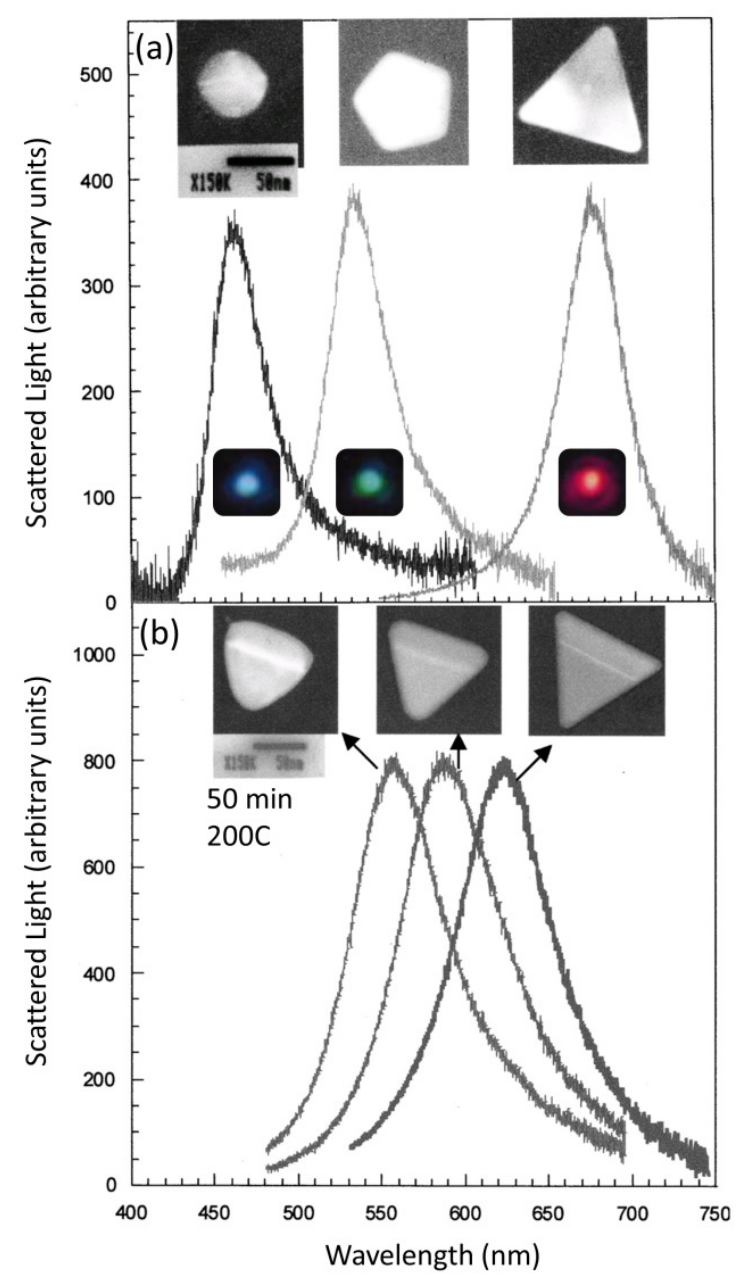

Figure 51: (a) Typical optical spectroscopy measurements of silver NPs showing the spectra of individual red, green, and blue particles. High-resolution TEM images of the corresponding NPs are shown above their respective spectra. The triangular-shaped NPs appear mostly red, NPs that form a pentagon appear green, and the blue NPs are spherical. The insets at the bottom correspond to an electronic camera color image of a typical sample of silver NPs as viewed under a dark field microscope. (b) Illustration of the particle shape and spectral modification by heating. The blue shift in the resonance peak by annealing the initial triangular silver NP in air at $200{ }^{\circ} \mathrm{C}$ for $30 \mathrm{~min}$ and $50 \mathrm{~min}$, respectively, is due to the NP shape change and rounding of the triangle corners. Adapted from Ref. [597].

It has been suggested that localized surface plasmon resonances (LSPR) may affect the catalytic activity of nanostructures. It is known that plasmon resonances could result in local NP heating [46; 606; 618] that could assist thermally-driven catalytic reactions [612]. However, it would be more favorable to avoid heating the NPs (both externally and localized via plasmons) in order to extend the catalyst lifetime. In an inert environment (e.g. vacuum), scattering phenomena (i.e. the re-emission of resonant photons) or charge carrier generation at the surface of nanostructures (i.e. energetic electrons and holes) are responsible for surface plasmon relaxations 
[619]. However, in the presence of adsorbates on the surface, a new pathway for surface plasmon relaxation, namely, the chemical interface damping (CID) should also be considered [620]. Following this mechanism, electron density transfers directly to the adsorbate. Both, the CID and the energetic electrons generated by the plasmon resonance could affect the catalytic properties of the NPs [591; 621]. One example of such photo-induced electron-driven catalytic reaction is shown in Figure 52. The rate of ethylene epoxidation is shown in Figure 52(a) in the presence and absence of visible light showing a 4-fold light-induced increase in the activity. Figure 52(b) shows the reaction rate as well as the plasmonic response intensity as a function of the light wavelength. A clear correlation between the plasmonic response and the reaction rate could be observed, with the highest activity at a wavelength of $\sim 600 \mathrm{~nm}$. Therefore, having shape- and size-selected NPs would result in similar LSPR for all NPs, which translates to a unique optimized wavelength for the whole catalyst.

Significant attention is being dedicated in the last couple of years to plasmon-induced catalytic processes, and further studies are needed to fully comprehend the mechanisms behind the exciting experimental observations that are being made and to ultimately take advantage of the plasmonic properties of metal NPs in catalysis. 

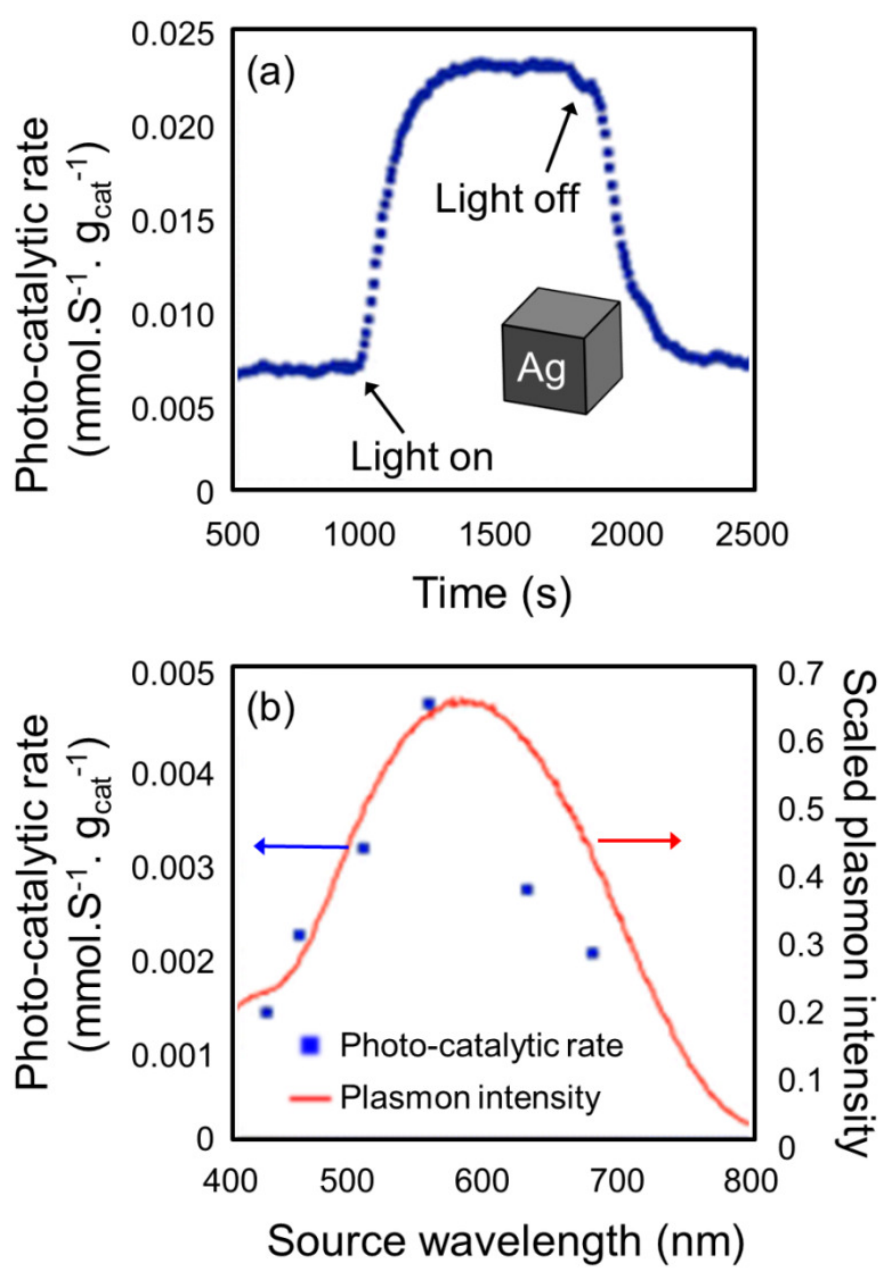

Figure 52: (a) The rate of ethylene epoxidation at $450 \mathrm{~K}$ in the dark and under visible illumination on $\mathrm{Ag}$ nanocubes supported on $\alpha-\mathrm{Al}_{2} \mathrm{O}_{3}$ [622]. (b) Photocatalytic rate as a function of the source wavelength at $470 \mathrm{~K}$. The solid line displays the plasmon intensity spectrum [623]. Adapted from $[591 ; 622 ; 623]$.

\section{5) Summary and Outlook}

Through the impressive leaps forward made in the last two decades in the field of nanocatalysis, it has become clear that detailed information on the physical/chemical properties of supported NP systems must be available for the rational design of the next generation of nanosized catalysts. Such NP systems must be representative of active catalysts, and must be sufficiently well-defined (e.g., with respect to size and shape) to serve as a basis for theoretical modeling. In addition, a synergistic combination of in-situ and in operando characterization techniques working under realistic reaction conditions (e.g. elevated temperatures and high pressure) must be available, since the working state of a NP catalyst might not be the state in which the catalyst was 
prepared, but a structural and/or chemical isomer that adapted to the particular reaction conditions. In order to address the complexity of real-world catalysts, a synergistic approach taking advantage of a variety of state-of-the-art experimental and theoretical methods must be undertaken. In this review, we have described some of the impressive developments that have been made in these areas.

Many serious challenges still remain in the areas of catalyst characterization and design. One such challenge is to design highly active metal NP catalysts using abundant, inexpensive metals which can replace precious metal catalysts. Additionally, there is an increasingly pressing need for catalyst development for energy production applications, such as in fuel cells or for the synthesis of chemicals and fuels. Further work is needed to design more active and selective catalysts for these reactions that are viable at an industrial scale. In the coming years, nanocatalysis research must meet these challenges by building on the work described in this report. Further advancements in developing new synthetic methods will allow increased control over NP structure and stability, allowing for new insights into NP structure-reactivity correlations. In addition, the development of higher resolution in situ and in operando measurement techniques capable of probing catalytic reactions at NP surfaces over an extended range of temperatures and pressures and harsh environmental conditions will give unprecedented insight into NP catalysts in their working state $[16 ; 21 ; 143 ; 213 ; 225 ; 301 ; 517 ; 624]$. Single-nanoparticle spectroscopic and catalytic characterization must also be further developed. Advancements in computational modelling of more realistic catalysts will also bring us closer to gaining a molecular-level view of a reaction at a NP surface when used to complement experimental studies. Such progress will bring us closer to realizing the goal of synthesizing highly effective, tailor-made, tunable nanoparticle catalysts. 


\section{Nomenclature}

AFM: $\quad$ Atomic Force Microscopy

BCC: $\quad$ Body Centered Cubic

BE: $\quad$ Binding Energy

$\mathrm{CN}$ : Coordination Number

DRIFTS: Diffuse Reflectance Infrared Spectroscopy

DFT: Density Functional Theory

DMF: Dimethylformamide

EXAFS: Extended x-ray Absorption Fine Structure

E-TEM: Environmental Transmission Electron Microscopy

FCC: $\quad$ Face Centered Cubic

FTIR: $\quad$ Fourier Transform Infrared spectroscopy

GISAXS: Grazing-Incidence Small-Angle X-ray Scattering

HAADF-STEM: $\quad$ High-Angle Annular Dark-Field Scanning Transmission Electron Microscopy

HOPG: $\quad$ Highly Oriented Pyrolytic Graphite

HRTEM: $\quad$ High Resolution Transmission Electron Microscopy

HXRD: Hard X-Ray Diffraction

CID: $\quad$ Chemical Interface Damping

IRAS: $\quad$ Infrared Reflection Absorption Spectroscopy

ITO: $\quad$ Indium Tin Oxide

KMC: $\quad$ Kinetic Monte Carlo

L: $\quad$ Langmuir

LEIS: $\quad$ Low Energy Ion Scattering

L-H: $\quad$ Langmuir-Hinshelwood

LSPR: Localized Surface Plasmon Resonance

ML: $\quad$ Mono-Layer

MD: $\quad$ Molecular Dynamics

MVK: $\quad$ Mars-van Krevelen

NP: $\quad$ Nanoparticle

PVP: $\quad$ Poly (Vinyl Pyrrolidone)

P2VP: Poly-2(Vinyl Pyrrolidone)

QMS: Quadrupole Mass Spectrometer

RT: $\quad$ Room Temperature 
SCAC: $\quad$ Single Crystal Adsorption Calorimetry

SFG: $\quad$ Sum Frequency Generation

SMSI: $\quad$ Strong Metal-Support Interaction

STO: $\quad$ Strontium Titanate, $\mathrm{SrTiO}_{3}$

STM: $\quad$ Scanning Tunneling Microscopy

STS: $\quad$ Scanning Tunneling Spectroscopy

SXRD: $\quad$ Surface X-Ray Diffraction

TEM: $\quad$ Transmission Electron Microscopy

TOF: $\quad$ Turnover Frequency

TPD: $\quad$ Temperature Programmed Desorption

TPR: Temperature Programmed Reduction

XANES: X-ray Absorption Near-Edge Spectroscopy

XAS: $\quad$ X-ray Absorption Spectroscopy

XPS: X-ray Photoelectron spectroscopy

XRD: $\quad$ X-Ray Diffraction

VOC: Volatile Organic Compounds

WBDF: Weak Beam Dark Field 


\section{Acknowledgement}

The authors would like to thank Hemma Mistry for her review of the present manuscript and helpful discussions. The financial support from the US National Science Foundation (NSFChemistry 1213182 and NSF-DMR-1006232) and the Office of Basic Energy Sciences of the US Department of Energy (DE-FG02-08ER15995) is greatly appreciated. This work was also supported by the Cluster of Excellence RESOLV at RUB (EXC 1069) funded by the Deutsche Forschungsgemeinschaft.

\section{References}

[1] Y. Li, Q. Liu, W. Shen, Dalton T. 40 (2011) 5811-5826.

[2] B. Roldan Cuenya, Accounts Chem. Res. 46 (2012) 1682-1691.

[3] S. Mostafa, F. Behafarid, J.R. Croy, L.K. Ono, L. Li, J.C. Yang, et al., J. Am. Chem. Soc. 132 (2010) 15714-15719.

[4] T.F. Jaramillo, S.H. Baeck, B. Roldan Cuenya, E.W. McFarland, J. Am. Chem. Soc. 125 (2003) 7148-7149.

[5] M. Valden, X. Lai, D.W. Goodman, Science 281 (1998) 1647-1650.

[6] M. Haruta, Catal. Today 36 (1997) 153-166.

[7] S. Xie, S.-I. Choi, X. Xia, Y. Xia, Current Opinion in Chemical Engineering 2 (2013) 142150.

[8] F. Zaera, Prog. Surf. Sci. 69 (2001) 1-98.

[9] F. Zaera, Chem. Soc. Rev. 42 (2013) 2746-2762.

[10] Z.H. Qin, M. Lewandowski, Y.N. Sun, S. Shaikhutdinov, H.J. Freund, J. Phys. Chem. C 112 (2008) 10209-10213.

[11] C.T. Campbell, J.C. Sharp, Y. Yao, E.M. Karp, T.L. Silbaugh, Faraday discussions 152 (2011) 227-239.

[12] S.K. Shaikhutdinov, R. Meyer, M. Naschitzki, M. Baumer, H.J. Freund, Catal. Lett. 86 (2003) 211-219.

[13] L. Ono, B. Yuan, H. Heinrich, B.R. Cuenya, J. Phys. Chem. C 114 (2010) 22119-22133.

[14] B. Roldan Cuenya, Thin Solid Films 518 (2010) 3127-3150.

[15] M. Ackermann, T. Pedersen, B. Hendriksen, O. Robach, S. Bobaru, I. Popa, et al., Phys. Rev. Lett. 95 (2005) 255505.

[16] L.R. Merte, M. Ahmadi, F. Behafarid, L.K. Ono, E. Lira, J. Matos, et al., ACS Catal. 3 (2013) 1460-1468.

[17] R. Ouyang, J.-X. Liu, W.-X. Li, J. Am. Chem. Soc. 135 (2012) 1760-1771.

[18] H. Mistry, F. Behafarid, S.R. Bare, B. Roldan Cuenya, ChemCatChem 6 (2014) 348-352.

[19] C. Mager-Maury, G. Bonnard, C. Chizallet, P. Sautet, P. Raybaud, ChemCatChem 3 (2011) 200-207. 
[20] J.C. Yang, M.W. Small, R.V. Grieshaber, R.G. Nuzzo, Chem. Soc. Rev. 41 (2012) 81798194.

[21] M.A. Bañares, Advanced Materials 23 (2011) 5293-5301.

[22] M.S. Chen, D.W. Goodman, Science 306 (2004) 252-255.

[23] H.-J. Freund, Top. Catal. 48 (2008) 137-144.

[24] M. Frank, M. Baumer, Phys. Chem. Chem. Phys. 2 (2000) 3723-3737.

[25] M. Baron, O. Bondarchuk, D. Stacchiola, S. Shaikhutdinov, H.J. Freund, J. Phys. Chem. C 113 (2009) 6042-6049.

[26] J.R. Kitchin, M.A. Barteau, J.G. Chen, Surf. Sci. 526 (2003) 323-331.

[27] F. Behafarid, B. Roldan Cuenya, Surf. Sci. 606 (2012) 908-918.

[28] S.T. Christensen, H. Feng, J.L. Libera, N. Guo, J.T. Miller, P.C. Stair, et al., Nano Lett. 10 (2010) 3047-3051.

[29] C. Detavernier, J. Dendooven, S. Pulinthanathu Sree, K.F. Ludwig, J.A. Martens, Chem. Soc. Rev. 40 (2011) 5242.

[30] I.J. Hsu, Y.C. Kimmel, X. Jiang, B.G. Willis, J.G. Chen, Chem. Commun. 48 (2012) 10631065.

[31] I.J. Hsu, D.A. Hansgen, B.E. McCandless, B.G. Willis, J.G. Chen, J. Phys. Chem. C 115 (2011) 3709-3715.

[32] H. Iddir, V. Komanicky, S. Ogut, H. You, P. Zapol, J. Phys. Chem. C 111 (2007) 1478214789.

[33] V. Komanicky, H. Iddir, K.C. Chang, A. Menzel, G. Karapetrov, D. Hennessy, et al., J. Am. Chem. Soc. 131 (2009) 5732-5733.

[34] A.S. Eppler, G. Rupprechter, L. Guczi, G.A. Somorjai, J. Phys. Chem. B 101 (1997) 9973.

[35] P.W. Jacobs, F.H. Ribeiro, G.A. Somorjai, S.J. Wind, Catal. Lett. 37 (1996) 131-136.

[36] A. Kosiorek, W. Kandulski, H. Glaczynska, M. Giersig, Small 1 (2005) 439-444.

[37] C.L. Haynes, R.P. Van Duyne, J. Phys. Chem. B 105 (2001) 5599-5611.

[38] A. Kosiorek, W. Kandulski, P. Chudzinski, K. Kempa, M. Giersig, Nano Lett. 4 (2004) 1359-1363.

[39] C.L. Haynes, A.D. McFarland, M.T. Smith, J.C. Hulteen, R.P. Van Duyne, J. Phys. Chem. B 106 (2002) 1898-1902.

[40] V. Johanek, M. Laurin, J. Hoffmann, S. Schauermann, A.W. Grant, B. Kasemo, et al., Surf. Sci. 561 (2004) L218-L224.

[41] G.A. Somorjai, Appl. Surf. Sci. 121 (1997) 1-19.

[42] Z. Song, J. Hrbek, R. Osgood, Nano Lett. 5 (2005) 1327-1332.

[43] E. Gross, M. Asscher, M. Lundwall, D.W. Goodman, J Phys. Chem. C 111 (2007) 16197.

[44] J.S. King, A. Wittstock, J. Biener, S.O. Kucheyev, Y.M. Wang, T.F. Baumann, et al., Nano Lett. 8 (2008) 2405-2409.

[45] A.M. Boies, J.T. Roberts, S.L. Girshick, B. Zhang, T. Nakamura, A. Mochizuki, Nanotechnology 20 (2009) 295604.

[46] D.A. Boyd, L. Greengard, M. Brongersma, M.Y. El-Naggar, D.G. Goodwin, Nano Lett. 6 (2006) 2592-2597. 
[47] R.G. Palgrave, I.P. Parkin, Gold Bull. 41 (2008) 66-69.

[48] L. Benz, X. Tong, P. Kemper, Y. Lilach, A. Kolmakov, H. Metiu, et al., J. Chem. Phys. 122 (2005) 081102.

[49] S. Pratontep, S.J. Carroll, C. Xirouchaki, M. Streun, R.E. Palmer, Rev. Sci. Instrum. 76 (2005).

[50] B. von Issendorff, R.E. Palmer, Rev. Sci. Instrum. 70 (1999) 4497-4501.

[51] I.M. Goldby, B. vonIssendorff, L. Kuipers, R.E. Palmer, Rev. Sci. Instrum. 68 (1997) 3327-3334.

[52] Z.W. Wang, R.E. Palmer, Nano Lett. 12 (2012) 91-95.

[53] S.H. Baeck, T.F. Jaramillo, G.D. Stucky, E.W. McFarland, Nano Lett. 2 (2002) 831.

[54] S.H. Baeck, T.F. Jaramillo, A. Kleinman-Shwarsetein, E.W. McFarland, Meas. Sci. Technol. 16 (2005) 54.

[55] J.V. Zoval, J. Lee, S. Gorer, R.M. Penner, J. Phys. Chem. B 102 (1998) 1166-1175.

[56] R.M. Stiger, S. Gorer, B. Craft, R.M. Penner, Langmuir 15 (1999) 790-798.

[57] L. Zhang, Z. Fang, G.C. Zhao, X.W. Wei, Int. J. Electrochem. Sci. 3 (2008) 746-754.

[58] R. Narayanan, M.A. El-Sayed, J. Phys. Chem. B 108 (2004) 5726-5733.

[59] S. Yamamuro, K. Sumiyama, Chem. Phys. Lett. 418 (2006) 166-169.

[60] C.-J. Jia, F. Schüth, Phys. Chem. Chem. Phys. 13 (2011) 2457.

[61] G.J. Hutchings, Gold Bull. 37 (2004) 3-11.

[62] T. Akita, M. Okumura, K. Tanaka, S. Tsubota, M. Haruta, J. Electron Microsc. 52 (2003) 119-124.

[63] M. Ichikawa, in: H.P. D.D. Eley, P.B. Weisz (Ed.), Advances in Catalysis, Academic Press Inc., San Diego, 1992.

[64] M.K. Carpenter, T.E. Moylan, R.S. Kukreja, M.H. Atwan, M.M. Tessema, J. Am. Chem. Soc. 134 (2012) 8535-8542.

[65] C. Cui, L. Gan, H.-H. Li, S.-H. Yu, M. Heggen, P. Strasser, Nano Lett. 12 (2012) 58855889.

[66] C. Cui, L. Gan, M. Heggen, S. Rudi, P. Strasser, Nat. Mater. 12 (2013) 765-771.

[67] M.R. Knecht, M.G. Weir, V.S. Myers, W.D. Pyrz, H. Ye, V. Petkov, et al., Chem. Mater. 20 (2008) 5218-5228.

[68] L. Jin, S.P. Yang, Q.W. Tian, H.X. Wu, Y.J. Cai, Mater. Chem. Phys. 112 (2008) 977-983.

[69] R.M. Crooks, M.Q. Zhao, L. Sun, V. Chechik, L.K. Yeung, Accounts Chem. Res. 34 (2001) 181-190.

[70] G. Kastle, H.G. Boyen, F. Weigl, G. Lengl, T. Herzog, P. Ziemann, et al., Adv. Funct. Mater. 13 (2003) 853-861.

[71] B. Roldan Cuenya, S.H. Baeck, T.F. Jaramillo, E.W. McFarland, J. Am. Chem. Soc. 125 (2003) 12928-12934.

[72] L.K. Ono, D. Sudfeld, B. Roldan Cuenya, Surf. Sci. 600 (2006) 5041-5050.

[73] J.R. Croy, S. Mostafa, H. Heinrich, B. Roldan Cuenya, Catal. Lett. 131 (2009) 21.

[74] C.-H. Lee, S.-T. Wong, T.-S. Lin, C.-Y. Mou, J. Phys. Chem. B 109 (2005) 775-784.

[75] F. Bedioui, Coord. Chem. Rev. 144 (1995) 39-68. 
[76] E.F. Murphy, D. Ferri, A. Baiker, S. Van Doorslaer, A. Schweiger, Inorg. Chem. 42 (2003) 2559-2571.

[77] S. Seelan, A.K. Sinha, D. Srinivas, S. Sivasanker, J. Mol. Catal. A: Chem. 157 (2000) 163171.

[78] S. Koner, K. Chaudhari, T.K. Das, S. Sivasanker, J. Mol. Catal. A: Chem. 150 (1999) 295297.

[79] R. Ganesan, B. Viswanathan, J. Phys. Chem. B 108 (2004) 7102-7114.

[80] Q. Zhang, I. Lee, J. Ge, F. Zaera, Y. Yin, Adv. Funct. Mater. 20 (2010) 2201-2214.

[81] F. Schüth, Chem. Mater. 26 (2014) 423-434.

[82] A. Kleibert, W. Rosellen, M. Getzlaff, J. Bansmann, Beilstein J. Nanotechnol. 2 (2011) 47 56.

[83] R.E. Palmer, S. Pratontep, H.G. Boyen, Nat. Mater. 2 (2003) 443-448.

[84] C. Xirouchaki, R.E. Palmer, Philos. Trans. A 362 (2004) 117-124.

[85] S. Vajda, M.J. Pellin, J.P. Greeley, C.L. Marshall, L.A. Curtiss, G.A. Ballentine, et al., Nat. Mater. 8 (2009) 213-216.

[86] F.J. Perez-Alonso, D.N. McCarthy, A. Nierhoff, P. Hernandez-Fernandez, C. Strebel, I.E.L. Stephens, et al., Angew. Chem. Int. Edit. 51 (2012) 4641-4643.

[87] K.M. Bratlie, H. Lee, K. Komvopoulos, P. Yang, G.A. Somorjai, Nano Lett. 7 (2007) 3097-3101.

[88] H. Lee, S.E. Habas, S. Kweskin, D. Butcher, G.A. Somorjai, P.D. Yang, Angew. Chem. Int. Edit. 45 (2006) 7824-7828.

[89] Y.W. Zhang, M.E. Grass, J.N. Kuhn, F. Tao, S.E. Habas, W.Y. Huang, et al., J. Am. Chem. Soc. 130 (2008) 5868-5869.

[90] C.J. Murphy, Science 298 (2002) 2139-2141.

[91] H. Lee, S.E. Habas, S. Kweskin, D. Butcher, G.A. Somorjai, P. Yang, Angew. Chem. Int. Edit. 118 (2006) 7988-7992.

[92] D. Mott, J. Galkowski, L. Wang, J. Luo, C.-J. Zhong, Langmuir 23 (2007) 5740-5745.

[93] Y. Chen, X. Gu, C.-G. Nie, Z.-Y. Jiang, Z.-X. Xie, C.-J. Lin, Chem. Commun. (2005) 4181.

[94] J. Ren, R.D. Tilley, Small 3 (2007) 1508-1512.

[95] F. Kim, S. Connor, H. Song, T. Kuykendall, P. Yang, Angew. Chem. Int. Edit. 116 (2004) 3759-3763.

[96] M. Grzelczak, J. Pérez-Juste, P. Mulvaney, L.M. Liz-Marzán, Chem. Soc. Rev. 37 (2008) 1783.

[97] T. Teranishi, R. Kurita, M. Miyake, J. Inorg. Organomet. P. 10 (2000) 145-156.

[98] S. Cheong, J.D. Watt, R.D. Tilley, Nanoscale 2 (2010) 2045.

[99] Y. Xia, Y. Xiong, B. Lim, S.E. Skrabalak, Angew. Chem. Int. Edit. 48 (2009) 60-103.

[100] F. Behafarid, B. Roldan Cuenya, Nano Lett. 11 (2011) 5290-5296.

[101] F. Zaera, Catal. Lett. 142 (2012) 501-516.

[102] M. Crespo-Quesada, A. Yarulin, M. Jin, Y. Xia, L. KiwiOMinsker, J. Am. Chem. Soc. 133 (2011) 12787-12794. 
[103] R. Narayanan, M.A. El-Sayed, Nano Lett. 4 (2004) 1343-1348.

[104] R. Xu, D. Wang, J. Zhang, Y. Li, Chem. Asian J. 1 (2006) 888-893.

[105] C.-H. Kuo, M.H. Huang, Nano Today 5 (2010) 106-116.

[106] J. Park, J. Joo, S.G. Kwon, Y. Jang, T. Hyeon, Angew. Chem. Int. Edit. 46 (2007) 46304660.

[107] V. Lablokov, S.K. Beaumont, S. Alayoglu, V.V. Pushkarev, C. Specht, J. Gao, et al., Nano Lett. 12 (2012) 3091-3096.

[108] J. Park, E. Lee, N.-M. Hwang, M. Kang, S.C. Kim, Y. Hwang, et al., Angew. Chem. Int. Edit. 117 (2005) 2932-2937.

[109] A.R. Tao, S. Habas, P. Yang, Small 4 (2008) 310-325.

[110] Y.N. Xia, M.S. Jin, H. Zhang, Z.X. Xie, Angew. Chem. Int. Edit. 50 (2011) 7850-7854.

[111] B. Roldan Cuenya, J.R. Croy, S. Mostafa, F. Behafarid, L. Li, Z. Zhang, et al., J. Am. Chem. Soc. 132 (2010) 8747-8756.

[112] L.K. Ono, J.R. Croy, H. Heinrich, B. Roldan Cuenya, J. Phys. Chem. C 115 (2011) 1685616866.

[113] F. Behafarid, L.K. Ono, S. Mostafa, J.R. Croy, G. Shafai, S. Hong, et al., Phys. Chem. Chem. Phys. 14 (2012) 11766.

[114] H. Mistry, F. Behafarid, E. Zhou, L.K. Ono, L. Zhang, B. Roldan Cuenya, ACS Catal. 4 (2014) 109-115.

[115] A. Naitabdi, L.K. Ono, F. Behafarid, B. Roldan Cuenya, J. Phys. Chem. C 113 (2009) 1433-1446.

[116] S.-W. Yeh, T.-L. Wu, K.-H. Wei, Nanotechnology 16 (2005) 683-687.

[117] J.A. Fan, K. Bao, L. Sun, J. Bao, V.N. Manoharan, P. Nordlander, et al., Nano Lett. 12 (2012) 5318-5324.

[118] Y.-H. La, M.P. Stoykovich, S.-M. Park, P.F. Nealey, Chem. Mater. 19 (2007) 4538-4544.

[119] J.V. Barth, G. Costantini, K. Kern, Nature 437 (2005) 671-679.

[120] A.J. Parker, P.A. Childs, R.E. Palmer, M. Brust, Appl. Phys. Lett. 74 (1999) 2833.

[121] D. Whang, S. Jin, C.M. Lieber, Nano Lett. 3 (2003) 951-954.

[122] J.-L. Li, J.-F. Jia, X.-J. Liang, X. Liu, J.-Z. Wang, Q.-K. Xue, et al., Phys. Rev. Lett. 88 (2002).

[123] T.A. Winningham, H.P. Gillis, D.A. Choutov, K.P. Martin, J.T. Moore, K. Douglas, Surf. Sci. 406 (1998) 221-228.

[124] Y. Pan, M. Gao, L. Huang, F. Liu, H.J. Gao, Appl. Phys. Lett. 95 (2009) 093106.

[125] S. Hoeppener, R. Maoz, S.R. Cohen, L.F. Chi, H. Fuchs, J. Sagiv, Adv. Mater. 14 (2002) 1036.

[126] D. Wang, H. Möhwalda, J. Mater. Chem. 14 (2004) 459-468.

[127] A. Goriachko, Y. He, M. Knapp, H. Over, M. Corso, T. Brugger, et al., Langmuir 23 (2007) 2928-2931.

[128] I. Brihuega, C.H. Michaelis, J. Zhang, S. Bose, V. Sessi, J. Honolka, et al., Surf. Sci. 602 (2008) L95-L99.

[129] A. N’Diaye, S. Bleikamp, P. Feibelman, T. Michely, Phys. Rev. Lett. 97 (2006) 215501. 
[130] N. Berdunov, G. Mariotto, K. Balakrishnan, S. Murphy, I.V. Shvets, Surf. Sci. 600 (2006) L287-L290.

[131] H. Hövel, I. Barke, Prog. Surf. Sci. 81 (2006) 53-111.

[132] Z. Yuan, R. Stephan, M.C. Hanf, J.M. Becht, C. Le Drian, M. Hugentobler, et al., Eur. Phys. J. D 63 (2011) 401-406.

[133] M. Limat, G. Fóti, M. Hugentobler, R. Stephan, W. Harbich, Catal. Today 146 (2009) 378385.

[134] Z. Yuan, M.C. Hanf, R. Stephan, F. Dulot, E. Denys, A. Florentin, et al., Sur. Interface Anal. 47 (2015) 82-86.

[135] M. Hugentobler, S. Bonanni, A. Sautier, W. Harbich, Eur. Phys. J. D 63 (2011) 215-220.

[136] P. Maury, M. Escalante, D.N. Reinhoudt, J. Huskens, Adv. Mater. 17 (2005) 2718-2723.

[137] L.C. Glangchai, M. Caldorera-Moore, L. Shi, K. Roy, J. Control. Release 125 (2008) 263272.

[138] A.M. Contreras, J. Grunes, X.M. Yan, A. Liddle, G.A. Somorjai, Catal. Lett. 100 (2005) 115-124.

[139] D. Qin, Y. Xia, B. Xu, H. Yang, C. Zhu, G.M. Whitesides, Adv. Mater. 11 (1999) 14331437.

[140] D.O. Shin, D.H. Lee, H.-S. Moon, S.-J. Jeong, J.Y. Kim, J.H. Mun, et al., Adv. Funct. Mater. 21 (2011) 250-254.

[141] M. Park, C. Harrison, P.M. Chaikin, R.A. Register, D.H. Adamson, Science 276 (1997) 1401-1404.

[142] S.-J. Jeong, G. Xia, B.H. Kim, D.O. Shin, S.-H. Kwon, S.-W. Kang, et al., Adv. Mater. 20 (2008) 1898-1904.

[143] F. Behafarid, B. Roldan Cuenya, Top. Catal. 56 (2013) 1542-1559.

[144] N.A. Gjostein, P. Wynblatt, Abstracts of Papers of the American Chemical Society (1974) 52-52.

[145] P. Wynblatt, N.A. Gjostein, Prog. Solid State Chem. 9 (1975) 21-58.

[146] M.J.J. Jak, C. Konstapel, A. van Kreuningen, J. Chrost, J. Verhoeven, J.W.M. Frenken, Surf. Sci. 474 (2001) 28-36.

[147] C.T. Campbell, J.R.V. Sellers, Faraday Discuss. 162 (2013) 9.

[148] A.K. Datye, Q. Xu, K.C. Kharas, J.M. McCarty, Catal. Today 111 (2006) 59-67.

[149] C.T. Campbell, S.C. Parker, D.E. Starr, Science 298 (2002) 811-814.

[150] B. Wiley, Y. Sun, B. Mayers, Y. Xia, Chem-Eur. J. 11 (2005) 454-463.

[151] S.M. Humphrey, M.E. Grass, S.E. Habas, K. Niesz, G.A. Somorjai, T.D. Tilley, Nano Lett. 7 (2007) 785-790.

[152] G.A. Somorjai, J.Y. Park, Top. Catal. 49 (2008) 126-135.

[153] C.J. Murphy, T.K. Sau, A.M. Gole, C.J. Orendorff, J. Gao, L. Gou, et al., J. Phys. Chem. B 109 (2005) 13857-13870.

[154] T.-H. Tran, T.-D. Nguyen, Colloids Surf. B Biointerfaces 88 (2011) 1-22.

[155] W.X. Niu, G.B. Xu, Nano Today 6 (2011) 265-285.

[156] J.Y. Chen, B. Lim, E.P. Lee, Y.N. Xia, Nano Today 4 (2009) 81-95. 
[157] S.S. Shankar, A. Rai, B. Ankamwar, A. Singh, A. Ahmad, M. Sastry, Nat. Mater. 3 (2004) 482-488.

[158] H. Song, F. Kim, S. Connor, G.A. Somorjai, P. Yang, J. Phys. Chem. B 109 (2005) 188193.

[159] T.A. Gschneidtner, Y.A.D. Fernandez, S. Syrenova, F. Westerlund, C. Langhammer, K. Moth-Poulsen, Langmuir 30 (2014) 3041-3050.

[160] W. Niu, L. Zhang, G. Xu, ACS Nano 4 (2010) 1987-1996.

[161] H.-L. Wu, C.-H. Kuo, M.H. Huang, Langmuir 26 (2010) 12307-12313.

[162] B. Mayers, X. Jiang, D. Sunderland, B. Cattle, Y. Xia, J. Am. Chem. Soc. 125 (2003) 13364-13365.

[163] L. Manna, D.J. Milliron, A. Meisel, E.C. Scher, A.P. Alivisatos, Nat. Mater. 2 (2003) 382385.

[164] K.-T. Yong, Y. Sahoo, K.R. Choudhury, M.T. Swihart, J.R. Minter, P.N. Prasad, Nano Lett. 6 (2006) 709-714.

[165] J. Zhu, S. Liu, O. Palchik, Y. Koltypin, A. Gedanken, Langmuir 16 (2000) 6396-6399.

[166] I. Ortac, D. Simberg, Y.-s. Yeh, J. Yang, B. Messmer, W.C. Trogler, et al., Nano Lett. (2014) 140206093348000.

[167] X. Teng, H. Yang, Nano Lett. 5 (2005) 885-891.

[168] M. Lattuada, T.A. Hatton, Nano Today 6 (2011) 286-308.

[169] L.D. Marks, Rep. Prog. Phys. 57 (1994) 603-649.

[170] M.L. Personick, M.R. Langille, J. Zhang, C.A. Mirkin, Nano Lett. 11 (2011) 3394-3398.

[171] A. Tao, P. Sinsermsuksakul, P. Yang, Angew. Chem. Int. Edit. 45 (2006) 4597-4601.

[172] F. Fievet, J. Lagier, B. Blin, B. Beaudoin, M. Figlarz, Solid State Ionics 32-33 (1989) 198205.

[173] Y. Sun, Y. Xia, Science 298 (2002) 2176-9.

[174] T.S. Ahmadi, Z.L. Wang, A. Henglein, M.A. El-Sayed, Chem. Mater. 8 (1996) 1161-1163.

[175] T.S. Ahmadi, Z.L. Wang, T.C. Green, A. Henglein, M.A. El-Sayed, Science 272 (1996) 1924-1925.

[176] J.M. Petroski, Z.L. Wang, T.C. Green, M.A. El-Sayed, J. Phys. Chem. B 102 (1998) 33163320.

[177] C. Wang, H. Daimon, T. Onodera, T. Koda, S. Sun, Angew. Chem. Int. Edit. 47 (2008) 3588-3591.

[178] N. Tian, Z.-Y. Zhou, S.-G. Sun, J. Phys. Chem. C 112 (2008) 19801-19817.

[179] W.A. Egli, A. Visintin, W.E. Triaca, A.J. Arvia, Appl. Surf. Sci. 68 (1993) 583-593.

[180] A. Visintin, J.C. Canullo, W.E. Triaca, A.J. Arvia, J. Electroanal. Chem. 239 (1988) 67-89.

[181] N. Tian, Z.-Y. Zhou, S.-G. Sun, Y. Ding, Z.L. Wang, Science 316 (2007) 732-735.

[182] Z.-Y. Zhou, N. Tian, Z.-Z. Huang, D.-J. Chen, S.-G. Sun, Faraday Discuss. 140 (2009) 81.

[183] P. Strasser, S. Koh, T. Anniyev, J. Greeley, K. More, C.F. Yu, et al., Nat. Chem. 2 (2010) 454-460.

[184] M. Ahmadi, F. Behafarid, C. Cui, P. Strasser, B. Roldan Cuenya, ACS Nano 7 (2013) 9195-9204. 
[185] Y. Wu, D. Wang, Z. Niu, P. Chen, G. Zhou, Y. Li, Angew. Chem. Int. Edit. 51 (2012) 12524-12528.

[186] M.A. Mahmoud, F. Saira, M.A. El-Sayed, Nano Lett. 10 (2010) 3764-3769.

[187] C.-H. Kuo, M.H. Huang, J. Am. Chem. Soc. 130 (2008) 12815-12820.

[188] I. Lee, F. Delbecq, R. Morales, M.A. Albiter, F. Zaera, Nature Mater. 8 (2009) 132-138.

[189] G. Wulff, Z. Krystallogr. Minera. 34 (1901) 449-530.

[190] C.R. Henry, Prog. Surf. Sci. 80 (2005) 92-116.

[191] C.M. Wang, D.R. Baer, J.E. Amonette, M.H. Engelhard, Y. Qiang, J. Antony, Nanotechnology 18 (2007) 255603.

[192] Y. Saito, K. Mihama, R. Uyeda, Jpn. J. Appl. Phys. 19 (1980) 1603-1610.

[193] A.S. Barnard, X.M. Lin, L.A. Curtiss, J. Phys. Chem. B 109 (2005) 24465-24472.

[194] T. Vystavel, S.A. Koch, G. Palasantzas, J.T.M. De Hosson, Appl. Phys. Lett. 86 (2005) 113113.

[195] A. Kleibert, A. Voitkans, K.-H. Meiwes-Broer, Phys. Status Solidi B 247 (2010) 10481055.

[196] H. Graoui, S. Giorgio, C.R. Enry, Philos. Mag. B 81 (2001) 1649-1658.

[197] H. Graoui, S. Giorgio, C.R. Henry, Surf. Sci. 417 (1998) 350-360.

[198] J.H. Ryu, S.S. Han, D.H. Kim, G. Henkelman, H.M. Lee, ACS Nano 5 (2011) 8515-8522.

[199] H.Y. Kim, G. Henkelman, J. Phys. Chem. Lett. 4 (2013) 216-221.

[200] P. Nolte, A. Stierle, N.Y. Jin-Phillip, N. Kasper, T.U. Schulli, H. Dosch, Science 321 (2008) 1654-1658.

[201] J.R. Croy, Supported Mono and Bimetallic Platinum and Iron Nanoparticles: Electronic, Structural, Catalytic, and Vibrational Properties, University of Central Florida, 2010.

[202] S. Giorgio, C.R. Henry, B. Pauwels, G. Van Tendeloo, Mat. Sci. Eng. a 297 (2001) $197-$ 202.

[203] R. Kaischew, Arbeitstagung Festkörperphysik; Wissenshaften: Dresden, Berlin, 1952, 8183.

[204] K. Paredis, L.K. Ono, F. Behafarid, Z.F. Zhang, J.C. Yang, A.I. Frenkel, et al., J. Am. Chem. Soc. 133 (2011) 13455-13464.

[205] F. Behafarid, B. Roldan Cuenya, J. Phys. Chem. Lett. 3 (2012) 608-612.

[206] B. Roldan Cuenya, M. Alcantra Ortigoza, L.K. Ono, F. Behafarid, S. Mostafa, J.R. Croy, et al., Phys. Rev. B 84 (2011) 245438.

[207] A. Naitabdi, F. Behafarid, B. Roldan Cuenya, Appl. Phys. Lett. 94 (2009) 083102.

[208] K.P. McKenna, A.L. Shluger, J. Phys. Chem. C 111 (2007) 18848-18852.

[209] M.A. Newton, Chem. Soc. Rev. 37 (2008) 2644-2657.

[210] M.A. El-Sayed, Accounts Chem. Res. 34 (2001) 257-264.

[211] M. Cabié, S. Giorgio, C.R. Henry, M.R. Axet, K. Philippot, B. Chaudret, J. Phys. Chem. C 114 (2010) 2160-2163.

[212] I. Lee, R. Morales, M.A. Albiter, F. Zaera, PNAS 105 (2008) 15241-15246.

[213] J. Matos, L.K. Ono, F. Behafarid, J.R. Croy, S. Mostafa, A.T. DeLaRiva, et al., Phys. Chem. Chem. Phys. 14 (2012) 11457. 
[214] P.L. Hansen, J.B. Wagner, S. Helveg, J.R. Rostrup-Nielsen, B.S. Clausen, H. Topsoe, Science 295 (2002) 2053-2055.

[215] J.K. Nørskov, T. Bligaard, J. Rossmeis, C.H. Christensen, Nature Chem. 1 (2009) 37-46.

[216] R.T. Vang, J.V. Lauritsen, E. Laegsgaard, F. Besenbacher, Chem. Soc. Rev. 37 (2008) 2191-2203.

[217] R. Si, M. Flytzani-Stephanopoulos, Angew. Chem. Int. Edit. 47 (2008) 2884-2887.

[218] F. Tao, M.E. Grass, Y. Zhang, D.R. Butcher, J.R. Renzas, Z. Liu, et al., Science 322 (2008) 932-934.

[219] M. Salmeron, R. Schlögl, Surf. Sci. Rep. 63 (2008) 169-199.

[220] J.K. Nørskov, T. Bligaard, B. Hvolbæk, F. Abild-Pedersen, I. Chorkendorff, C.H. Christensen, Chem. Soc. Rev. 37 (2008) 2163-2171.

[221] F. Mittendorfer, N. Seriani, O. Dubay, G. Kresse, Phys. Rev. B 76 (2007) 233413.

[222] R.B. Getman, W.F. Schneider, ChemCatChem 2 (2010) 1450-1460.

[223] S.M. Oxford, P.L. Lee, P.J. Chupas, K.W. Chapman, M.C. Kung, H.H. Kung, J. Phys. Chem. C 114 (2010) 17085-17091.

[224] B.M. Weckhuysen, Angew. Chem. Int. Edit. 48 (2009) 4910-4943.

[225] M.A. Newton, C. Belver-Coldeira, A. Martínez-Arias, M. Fernández-García, Nat. Mater. 6 (2007) 528-532.

[226] M.A. Newton, M. Di Michiel, A. Kubacka, M. Fernandez-Garcia, J. Am. Chem. Soc. 132 (2010) 4540-4541.

[227] A.C. Shi, R.I. Masel, J. Catal. 120 (1989) 421-431.

[228] J. Hrbek, F.M. Hoffmann, J.B. Park, P. Liu, D. Stacchiola, Y.S. Hoo, et al., J. Am. Chem. Soc. 130 (2008) 17272-17273.

[229] W.H. Lee, K.R. Vanloon, V. Petrova, J.B. Woodhouse, C.M. Loxton, R.I. Masel, J. Catal. 126 (1990) 658-670.

[230] J.A. Enterkin, K.R. Poeppelmeier, L.D. Marks, Nano Lett. 11 (2011) 993-997.

[231] J. Yang, W. Hu, S. Chen, J. Tang, J. Phys. Chem. C 113 (2009) 21501-21505.

[232] S. Giorgio, M. Cabie, C.R. Henry, Gold Bull. 41 (2008) 167-173.

[233] C.T. Campbell, Science 306 (2004) 234-235.

[234] H.-J. Freund, Chem. Eur. J. 16 (2010) 9384-9397.

[235] L.C. Grabow, M. Mavrikakis, Angew. Chem. Int. Edit. 47 (2008) 7390-7392.

[236] E. Roduner, Chem. Soc. Rev. 35 (2006) 583.

[237] E. Roduner, Nanoscopic Materials: size-dependent phenomena, Cambridge : RSC Pub., 2006.

[238] C.N.R. Rao, G.U. Kulkarni, P.J. Thomas, P.P. Edwards, Chem-Eur. J. 8 (2002) 29-35.

[239] C.N.R. Rao, A. Müller, A.K. Cheetham, The chemistry of nanomaterials : synthesis, properties and applications in 2 volumes, Wiley-VCH, Weinheim, 2004.

[240] S.C. Parker, C.T. Campbell, Top. Catal. 44 (2007) 3-13.

[241] J.-H. Fischer-Wolfarth, J.A. Farmer, J.M. Flores-Camacho, A. Genest, I.V. Yudanov, N. Rösch, et al., Phys. Rev. B 81 (2010) 241416.

[242] D.C. Meier, D.W. Goodman, J. Am. Chem. Soc. 126 (2004) 1892-1899. 
[243] M. Peter, J.M. Flores Camacho, S. Adamovski, L.K. Ono, K.-H. Dostert, C.P. O'Brien, et al., Angew. Chem. Int. Edit. 52 (2013) 5175-5179.

[244] M. Neumaier, F. Weigend, O. Hampe, M.M. Kappes, J. Chem. Phys. 122 (2005) 104702.

[245] I.V. Yudanov, A. Genest, S. Schauermann, H.-J. Freund, N. Rösch, Nano Lett. 12 (2012) 2134-2139.

[246] S. Penner, P. Bera, S. Pedersen, L.T. Ngo, J.J.W. Harris, C.T. Campbell, J. Phys. Chem. B 110 (2006) 24577-24584.

[247] E.S. Putna, J.M. Vohs, R.J. Gorte, Surf. Sci. 391 (1997) L1178-L1182.

[248] C. Lemire, R. Meyer, S. Shaikhutdinov, H.J. Freund, Angew. Chem. Int. Edit. 43 (2004) 118.

[249] M.N. Padilla-Serrano, F.J. Maldonado-Hódar, C. Moreno-Castilla, Appl. Catal. B-Environ. 61 (2005) 253-258.

[250] V.A. Bondzie, S.C. Parker, C.T. Campbell, J. Vac. Sci. Technol. A 17 (1999) 1717-1720.

[251] V.A. Bondzie, S.C. Parker, C.T. Campbell, Catal. Lett. 63 (1999) 143-151.

[252] L.K. Ono, B. Roldan Cuenya, J. Phys. Chem. C 112 (2008) 18543-18550.

[253] Z.H. Qin, M. Lewandowski, Y.N. Sun, S. Shaikhutdinov, H.J. Freund, J. Phys. Condens. Matter. 21 (2009) 134019.

[254] S. Shaikhutdinov, M. Heemeier, J. Hoffmann, I. Meusel, B. Richter, M. Bäumer, et al., Surf. Sci. 501 (2002) 270-281.

[255] C. Lemire, R. Meyer, S.K. Shaikhutdinov, H.J. Freund, Surf. Sci. 552 (2004) 27-34.

[256] L.K. Ono, B. Roldán-Cuenya, Catal. Lett. 113 (2007) 86-94.

[257] J.L. Falconer, R.J. Madix, Surf. Sci. 48 (1975) 393-405.

[258] P. Chou, M.A. Vannice, J. Catal. 104 (1987) 17-30.

[259] C.R. Henry, C. Chapon, C. Goyhenex, R. Monot, Surf. Sci. 272 (1992) 283-288.

[260] C.R. Henry, Surf. Sci. Rep. 31 (1998) 231-325.

[261] C. Duriez, C.R. Henry, C. Chapon, Surf. Sci. 253 (1991) 190-204.

[262] I. Stara, V. Matolin, Surf. Sci. 313 (1994) 99-106.

[263] M. Peter, S. Adamovsky, J.M. Flores Camacho, S. Schauermann, Faraday Discuss. 162 (2013) 341.

[264] J.M. Flores-Camacho, J.H. Fischer-Wolfarth, M. Peter, C.T. Campbell, S. Schauermann, H.J. Freund, Phys. Chem. Chem. Phys. 13 (2011) 16800.

[265] G. Sitja, S.L. Moal, M. Marsault, G. Hamm, F. Leroy, C.R. Henry, Nano Lett. 13 (2013) 1977-1982.

[266] Y.Y. Yeo, L. Vattuone, D.A. King, J. Chem. Phys. 106 (1997) 1990.

[267] V. Matolin, E. Gillet, Surf. Sci. 238 (1990) 75-82.

[268] H. Conrad, G. Ertl, J. Koch, E.E. Latta, Surf. Sci. 43 (1974) 462-480.

[269] M. Mavrikakis, P. Stoltze, J.K. Norskov, Catal. Lett. 64 (2000) 101-106.

[270] M. Mavrikakis, B. Hammer, J. Nørskov, Phys. Rev. Lett. 81 (1998) 2819-2822.

[271] M. Klimenkov, S. Nepijko, H. Kuhlenbeck, M. Bäumer, R. Schlögl, H.J. Freund, Surf. Sci. 391 (1997) 27-36. 
[272] J.T. Miller, A.J. Kropf, Y. Zha, J.R. Regalbuto, L. Delannoy, C. Louis, et al., J. Catal. 240 (2006) 222-234.

[273] S. Krüger, S. Vent, F. Nörtemann, M. Staufer, N. Rösch, J. Chem. Phys. 115 (2001) 2082.

[274] W.J. Huang, R. Sun, J. Tao, L.D. Menard, R.G. Nuzzo, J.M. Zuo, Nat. Mater. 7 (2008) 308-313.

[275] S. Rives, A. Catherinot, F. Dumas-Bouchiat, C. Champeaux, A. Videcoq, R. Ferrando, Phys. Rev. B 77 (2008).

[276] E.Z. daSilva, A. Antonelli, Phys. Rev. B 54 (1996) 17057-17060.

[277] F. Behafarid, J. Matos, S. Hong, L. Zhang, T.S. Rahman, B. Roldan Cuenya, ACS Nano (2014) 140128093714001.

[278] C.-M. Lin, T.-L. Hung, Y.-H. Huang, K.-T. Wu, M.-T. Tang, C.-H. Lee, et al., Phys. Rev. B 75 (2007).

[279] P.M. Diehm, P. Ágoston, K. Albe, ChemPhysChem 13 (2012) 2443-2454.

[280] S. Tsunekawa, K. Ishikawa, Z.Q. Li, Y. Kawazoe, A. Kasuya, Phys. Rev. Lett. 85 (2000) 3440-3443.

[281] L. Wu, H. Wiesmann, A. Moodenbaugh, R. Klie, Y. Zhu, D. Welch, et al., Phys. Rev. B 69 (2004).

[282] A. Balerna, E. Bernieri, P. Picozzi, A. Reale, S. Santucci, E. Burattini, et al., Phys. Rev. B 31 (1985) 5058-5065.

[283] M. Marcus, M. Andrews, J. Zegenhagen, A. Bommannavar, P. Montano, Phys. Rev. B 42 (1990) 3312-3316.

[284] D. Zanchet, H. Tolentino, M.C. Martins Alves, O.L. Alves, D. Ugarte, Chem. Phys. Lett. 323 (2000) 167-172.

[285] L.D. Menard, H. Xu, S.-P. Gao, R.D. Twesten, A.S. Harper, Y. Song, et al., J. Phys. Chem. B 110 (2006) 14564-14573.

[286] J.A. vanBokhoven, J.T. Miller, J. Phys. Chem. C 111 (2007) 9245-9249.

[287] R. Lamber, S. Wetjen, N. Jaeger, Phys. Rev. B 51 (1995) 10968-10971.

[288] I.V. Yudanov, M. Metzner, A. Genest, N. Rösch, J. Phys. Chem. C 112 (2008) 2026920275.

[289] I.V. Yudanov, R. Sahnoun, K.M. Neyman, N. Rösch, J. Hoffmann, S. Schauermann, et al., J. Phys. Chem. B 107 (2003) 255-264.

[290] R. Naumann d'Alnoncourt, M. Friedrich, E. Kunkes, D. Rosenthal, F. Girgsdies, B. Zhang, et al., J. Catal. 317 (2014) 220-228.

[291] M. Boudart, Adv. Catal. 20 (1969) 153-166.

[292] R.M. Rioux, H. Song, J.D. Hoefelmeyer, P. Yang, G.A. Somorjai, J. Phys. Chem. B 109 (2005) 2192-2202.

[293] K.R. McCrea, G.A. Somorjai, J. Mol. Catal. A: Chem. 163 (2000) 43-53.

[294] C.-K. Tsung, J.N. Kuhn, W. Huang, C. Aliaga, L.-I. Hung, G.A. Somorjai, et al., J. Am. Chem. Soc. 131 (2009) 5816-5822.

[295] P. Panagiotopoulou, D.I. Kondarides, Catal. Today 112 (2006) 49-52.

[296] P. Panagiotopoulou, D.I. Kondarides, J. Catal. 225 (2004) 327-336. 
[297] K.M. Bratlie, C.J. Kliewer, G.A. Somorjai, J. Phys. Chem. B 110 (2006) 17925-17930.

[298] J.N. Kuhn, W. Huang, C.-K. Tsung, Y. Zhang, G.A. Somorjai, J. Am. Chem. Soc. 130 (2008) 14026-14027.

[299] H. Song, R.M. Rioux, J.D. Hoefelmeyer, R. Komor, K. Niesz, M. Grass, et al., J. Am. Chem. Soc. 128 (2006) 3027-3037.

[300] G. Wang, Y. Morikawa, T. Matsumoto, J. Nakamura, J. Phys. Chem. B 110 (2006) 9-11.

[301] E. Lira, L.R. Merte, F. Behafarid, L.K. Ono, L. Zhang, B. Roldan Cuenya, ACS Catal. (2014).

[302] S.M. Davis, G.A. Somorjai, J. Catal. 65 (1980) 78-83.

[303] F.H. Ribeiro, A.E. Schach Von Wittenau, C.H. Bartholomew, G.A. Somorjai, Catal. Rev. 39 (1997) 49-76.

[304] M. Stamatakis, Y. Chen, D.G. Vlachos, J. Phys. Chem. C 115 (2011) 24750-24762.

[305] P.S. Cremer, X. Su, Y.R. Shen, G.A. Somorjai, J. Am. Chem. Soc. 118 (1996) 2942-2949.

[306] F. Gao, D.W. Goodman, Annu. Rev. Phys. Chem. 63 (2012) 265-286.

[307] M. Salciccioli, Y. Chen, D.G. Vlachos, Ind. Eng. Chem. Res. 50 (2011) 28-40.

[308] Z. Nomikou, M.A. Van Hove, G.A. Somorjai, Langmuir 12 (1996) 1251-1256.

[309] H. Hirano, K.-I. Tanaka, J. Catal. 133 (1992) 461-466.

[310] H. Nakano, I. Nakamura, T. Fujitani, J. Nakamura, J. Phys. Chem. B 105 (2001) 13551365 .

[311] C. Stegelmann, A. Andreasen, C.T. Campbell, J. Am. Chem. Soc. 131 (2009) 8077-8082.

[312] J.R. Croy, S. Mostafa, J. Liu, Y.-h. Sohn, B. Roldan Cuenya, Catal. Lett. 118 (2007) 1-7.

[313] Y. Lei, F. Mehmood, S. Lee, J. Greeley, B. Lee, S. Seifert, et al., Science 328 (2010) 224228.

[314] W.P. Zhou, A. Lewera, R. Larsen, R.I. Masel, P.S. Bagus, A. Wieckowski, J. Phys. Chem. B 110 (2006) 13393-13398.

[315] S.H. Joo, J.Y. Park, J.R. Renzas, D.R. Butcher, W. Huang, G.A. Somorjai, Nano Lett. 10 (2010) 2709-2713.

[316] A. Boubnov, S. Dahl, E. Johnson, A.P. Molina, S.B. Simonsen, F.M. Cano, et al., Appl. Catal. B-Environ. 126 (2012) 315-325.

[317] J.A. Rodriguez, P. Liu, J. Hrbek, J. Evans, M. Pérez, Angew. Chem. Int. Edit. 46 (2007) 1329-1332.

[318] M. Valden, S. Pak, X. Lai, D.W. Goodman, Catal. Lett. 56 (1998) 7-10.

[319] J. Jia, K. Haraki, J.N. Kondo, K. Domen, K. Tamaru, J. Phys. Chem. B 104 (2000) 1115311156.

[320] R. Reske, H. Mistry, F. Behafarid, B. Roldan Cuenya, P. Strasser, J. Am. Chem. Soc. (2014) DOI: $10.1021 /$ ja500328k.

[321] T. Hayashi, K. Tanaka, M. Haruta, J. Catal. 178 (1998) 566-575.

[322] T. Akita, K. Tanaka, S. Tsubota, M. Haruta, J. Electron. Microsc. 49 (2000) 657-662.

[323] X.E. Verykios, F.P. Stein, R.W. Coughlin, J. Catal. 66 (1980) 368-382.

[324] J.K. Nørskov, T. Bligaard, A. Logadottir, S. Bahn, L.B. Hansen, M. Bollinger, et al., J. Catal. 209 (2002) 275-278. 
[325] M.G. Evans, M. Polanyi, T. Faraday. Soc. 34 (1938) 11-24.

[326] J.N. Bronsted, Chem. Rev. 5 (1928) 231-338.

[327] S. Wang, V. Vorotnikov, J.E. Sutton, D.G. Vlachos, ACS Catal. 4 (2014) 604-612.

[328] P. Ferrin, D. Simonetti, S. Kandoi, E. Kunkes, J.A. Dumesic, J.K. Nørskov, et al., J. Am. Chem. Soc. 131 (2009) 5809-5815.

[329] T. Bligaard, J.K. Nørskov, S. Dahl, J. Matthiesen, C.H. Christensen, J. Sehested, J. Catal. 224 (2004) 206-217.

[330] J. Greeley, I.E.L. Stephens, A.S. Bondarenko, T.P. Johansson, H.A. Hansen, T.F. Jaramillo, et al., Nature Chem. 1 (2009) 552-556.

[331] V. Stamenkovic, B.S. Mun, K.J.J. Mayrhofer, P.N. Ross, N.M. Markovic, J. Rossmeisl, et al., Angew. Chem. Int. Edit. 118 (2006) 2963-2967.

[332] C.J.H. Jacobsen, S. Dahl, B.S. Clausen, S. Bahn, A. Logadottir, J.K. Nørskov, J. Am. Chem. Soc. 123 (2001) 8404-8405.

[333] P. Schmitz, R. Kudla, A. Drews, A. Chen, C. Lowema, R. McCabe, et al., Appl. Catal. BEnviron. 67 (2006) 246-256.

[334] T. Bligaard, K. Honkala, A. Logadottir, J.K. Nørskov, S. Dahl, C.J.H. Jacobsen, J. Phys. Chem. B 107 (2003) 9325-9331.

[335] A. Logadottir, T.H. Rod, J.K. Nørskov, B. Hammer, S. Dahl, C.J.H. Jacobsen, J. Catal. 197 (2001) 229-231.

[336] O. Exner, Collect. Czech. Chem. Commun. 38 (1973) 799-812.

[337] F.H. Constable, Proc. R. Soc. Lond. A 108 (1925) 355-378.

[338] G.A. Somorjai, Y. Li, Introduction to surface chemistry and catalysis, Wiley, Hoboken, N.J., 2010.

[339] P.J. Barrie, Phys. Chem. Chem. Phys. 14 (2012) 318.

[340] P.J. Barrie, Phys. Chem. Chem. Phys. 14 (2012) 327.

[341] O. Exner, Nature 201 (1964) 488-490.

[342] W. Linert, Inorg. Chim. Acta 141 (1988) 233-242.

[343] W. Linert, R.F. Jameson, Chem. Soc. Rev. 18 (1989) 477.

[344] R.R. Krug, Ind. Eng. Chem. Fundamen. 19 (1980) 50-59.

[345] A. Yelon, E. Sacher, W. Linert, Phys. Chem. Chem. Phys. 14 (2012) 8232.

[346] E. Peacock-López, H. Suhl, Phys. Rev. B 26 (1982) 3774-3782.

[347] M. Boudart, G. Djéga-Mariadassou, Kinetics of heterogeneous catalytic reactions, Princeton University Press, Princeton, N.J., 1984.

[348] N. Koga, Thermochim. Acta 244 (1994) 1-20.

[349] L. Liu, Q.-X. Guo, Chem. Rev. 101 (2001) 673-696.

[350] L.E. Murillo, A.M. Goda, J.G. Chen, J. Am. Chem. Soc. 129 (2007) 7101-7105.

[351] J.R. Kitchin, N.A. Khan, M.A. Barteau, J.G. Chen, B. Yakshinskiy, T.E. Madey, Surf. Sci. 544 (2003) 295-308.

[352] J.G. Chen, C.A. Menning, M.B. Zellner, Surf. Sci. Rep. 63 (2008) 201-254.

[353] C.A. Menning, H.H. Hwu, J.G. Chen, J. Phys. Chem. B 110 (2006) 15471-15477. 
[354] W. Yu, M.D. Porosoff, J.G. Chen, Chem. Rev. 112 (2012) 5780-5817.

[355] S. Linic, J. Jankowiak, M.A. Barteau, J. Catal. 224 (2004) 489-493.

[356] B. Hammer, J.K. Norskov, Nature 376 (1995) 238-240.

[357] M. Haruta, T. Kobayashi, H. Sano, N. Yamada, Chem. Lett. (1987) 405-408.

[358] M. Haruta, M. Daté, Appl. Catal. A-Gen. 222 (2001) 427-437.

[359] S. Lee, C. Fan, T. Wu, S. Anderson, J. Am. Chem. Soc. 126 (2004) 5682-5683.

[360] R. Zanella, S. Giorgio, C.-H. Shin, C.R. Henry, C. Louis, J. Catal. 222 (2004) 357-367.

[361] O. Meerson, G. Sitja, C.R. Henry, Eur. Phys. J. D 34 (2005) 119-124.

[362] D. Andreeva, V. Idakiev, T. Tabakova, A. Andreev, R. Giovanoli, Appl. Catal. A-Gen. 134 (1996) 275-283.

[363] A. Ueda, M. Haruta, Gold Bull. 32 (1999) 3-11.

[364] S.K. Klitgaard, A.T. Riva, S. Helveg, R.M. Werchmeister, C.H. Christensen, Catal. Lett. 126 (2008) 213-217.

[365] G.J. Hutchings, Catal. Today 100 (2005) 55-61.

[366] D.T. Thompson, Appl. Catal. A-Gen. 243 (2003) 201-205.

[367] G.C. Bond, D.T. Thompson, Catal. Rev. 41 (1999) 319-388.

[368] M. Haruta, J. New Mater. Electrochem. Syst. 7 (2004) 163-172.

[369] N. Lopez, T.V.W. Janssens, B.S. Clausen, Y. Xu, M. Mavrikakis, T. Bligaard, et al., J. Catal. 223 (2004) 232-235.

[370] T.V. Choudhary, D.W. Goodman, Appl. Catal. A-Gen. 291 (2005) 32-36.

[371] A. Cho, Science 299 (2003) 1684.

[372] J.H. Pazmiño, J.T. Miller, S.S. Mulla, W. Nicholas Delgass, F.H. Ribeiro, J. Catal. 282 (2011) 13-24.

[373] K. Paredis, L.K. Ono, S. Mostafa, L. Li, Z.F. Zhang, J.C. Yang, et al., J. Am. Chem. Soc. 133 (2011) 6728-6735.

[374] Y. Volokitin, J. Sinzig, L.J. de Jongh, G. Schmid, M.N. Vargaftik, I.I. Moiseevi, Nature 384 (1996) 621-623.

[375] L. Yanhong, W. Dejun, Z. Qidong, Y. Min, Z. Qinglin, J. Phys. Chem. B 108 (2004) 32023206.

[376] G. Mills, M.S. Gordon, H. Metiu, J. Chem. Phys. 118 (2003) 4198-4205.

[377] A.W. Snow, H. Wohltjen, Chem. Mater. 10 (1998) 947-949.

[378] C.N.R. Rao, G.U. Kulkarni, P.J. Thomas, P.P. Edwards, Chem. Soc. Rev. 29 (2000) 27-35.

[379] D. Bazin, D. Sayers, J.J. Rehr, C. Mottet, J. Phys. Chem. B 101 (1997) 5332-5336.

[380] H. Häkkinen, M. Moseler, O. Kostko, N. Morgner, M. Hoffmann, B. v. Issendorff, Phys. Rev. Lett. 93 (2004) 093401.

[381] S. Chen, R.S. Ingram, M.J. Hostetler, J.J. Pietron, R.W. Murray, T.G. Schaaff, et al., Science 280 (1998) 2098-2101.

[382] L. Li, A.H. Larsen, N.A. Romero, V.A. Morozov, C. Glinsvad, F. Abild-Pedersen, et al., J. Phys. Chem. Lett. 4 (2013) 222-226.

[383] R. Kubo, J. Phys. Soc. Jpn. 17 (1962) 975-986. 
[384] P.P. Edwards, R.L. Johnston, C.N.R. Rao, in, Wiley-VCH Verlag GmbH, , Weinheim, Germany, 1999, pp. 1454-1481.

[385] A. Kawabata, R. Kubo, J. Phys. Soc. Jpn. 21 (1966) 1765-\&.

[386] H.G. Boyen, G. Kastle, F. Weigl, B. Koslowski, C. Dietrich, P. Ziemann, et al., Science 297 (2002) 1533-1536.

[387] C. Xu, X. Lai, G. Zajac, D. Goodman, Phys. Rev. B 56 (1997) 13464-13482.

[388] A. Naitabdi, L.K. Ono, B. Roldan Cuenya, Appl. Phys. Lett. 89 (2006) 043101.

[389] J. Kleis, J. Greeley, N.A. Romero, V.A. Morozov, H. Falsig, A.H. Larsen, et al., Catal. Lett. 141 (2011) 1067-1071.

[390] T.V.W. Janssens, B.S. Clausen, B. Hvolbæk, H. Falsig, C.H. Christensen, T. Bligaard, et al., Top. Catal. 44 (2007) 15-26.

[391] A.D. Karmazyn, V. Fiorin, S.J. Jenkins, D.A. King, Surf. Sci. 538 (2003) 171-183.

[392] G. Ertl, M. Neumann, K.M. Streit, Surf. Sci. 64 (1977) 393-410.

[393] M.G. Mason, Phys. Rev. B 27 (1983) 748-762.

[394] P. Zhang, T.K. Sham, Phys. Rev. Lett. 90 (2003) 245502.

[395] G.K. Wertheim, S.B. DiCenzo, S.E. Youngquist, Phys. Rev. Lett. 51 (1983) 2310-2313.

[396] Z.X. Yang, R.Q. Wu, Phys. Rev. B 67 (2003) 081403.

[397] H.G. Boyen, T. Herzog, G. Kästle, F. Weigl, P. Ziemann, J. Spatz, et al., Phys. Rev. B 65 (2002) 075412.

[398] O. Lopez-Acevedo, K.A. Kacprzak, J. Akola, H. Häkkinen, Nat. Chem. 2 (2010) 329-334.

[399] W.E. Kaden, T. Wu, W.A. Kunkel, S.L. Anderson, Science 326 (2009) 826-829.

[400] S. Abbet, A. Sanchez, U. Heiz, W.D. Schneider, A.M. Ferrari, G. Pacchioni, et al., J. Am. Chem. Soc. 122 (2000) 3453-3457.

[401] S. Proch, M. Wirth, H.S. White, S.L. Anderson, J. Am. Chem. Soc. 135 (2013) 3073-3086.

[402] S. Abbet, A. Sanchez, U. Heiz, W.-D. Schneider, J. Catal. 198 (2001) 122-127.

[403] U. Heiz, A. Sanchez, S. Abbet, W.D. Schneider, J. Am. Chem. Soc. 121 (1999) 3214-3217.

[404] U. Landman, B. Yoon, C. Zhang, U. Heiz, M. Arenz, Top. Catal. 44 (2007) 145-158.

[405] U. Heiz, F. Vanolli, A. Sanchez, W.D. Schneider, J. Am. Chem. Soc. 120 (1998) 96689671.

[406] W.E. Kaden, W.A. Kunkel, F.S. Roberts, M. Kane, S.L. Anderson, J. Chem. Phys. 136 (2012) 204705.

[407] S. Mori, Y. Shitara, Appl. Surf. Sci. 78 (1994) 269-273.

[408] G.A. Somorjai, K.R. McCrea, J. Zhu, Top. Catal. 18 (2002) 157-166.

[409] G.A. Somorjai, Chem. Rev. 96 (1996) 1223-1236.

[410] M. Boronat, A. Corma, F. Illas, J. Radilla, T. Ródenas, M.J. Sabater, J. Catal. 278 (2011) 50-58.

[411] I. Herraiz-Cardona, E. Ortega, J.G. Antón, V. Pérez-Herranz, Int. J. Hydrogen Energ. 36 (2011) 9428-9438.

[412] J.M. Gottfried, K.J. Schmidt, S.L.M. Schroeder, K. Christmann, Surf. Sci. 536 (2003) 206224. 
[413] H. Liu, P. He, Z. Li, J. Li, Nanotechnology 17 (2006) 2167-2173.

[414] C. Ruggiero, P. Hollins, Surf. Sci. 377-379 (1997) 583.

[415] A. Stroppa, F. Mittendorfer, J.N. Andersen, G. Parteder, F. Allegretti, S. Surnev, et al., J. Phys. Chem. C 113 (2009) 942-949.

[416] C. Ruggiero, P. Hollins, J. Chem. Soc. Faraday T. 92 (1996) 4829-4834.

[417] J. Kim, E. Samano, B.E. Koel, J. Phys. Chem. B 110 (2006) 17512-17517.

[418] C.J. Weststrate, E. Lundgren, J.N. Andersen, E.D.L. Rienks, A.C. Gluhoi, J.W. Bakker, et al., Surf. Sci. 603 (2009) 2152-2157.

[419] V. Komanicky, A. Menzel, H. You, J. Phys. Chem. B 109 (2005) 23550-23557.

[420] N.M. Markovic, H.A. Gasteiger, P.N. Ross, J. Phys. Chem. 99 (1995) 3411-3415.

[421] N.M. Marković, R.R. Adžić, B.D. Cahan, E.B. Yeager, J. Electroanal. Chem. 377 (1994) 249-259.

[422] S. Kondo, M. Nakamura, N. Maki, N. Hoshi, J. Phys. Chem. C 113 (2009) 12625-12628.

[423] M.K. Oudenhuijzen, J.A. van Bokhoven, J.T. Miller, D.E. Ramaker, D.C. Koningsberger, J. Am. Chem. Soc. 127 (2005) 1530-1540.

[424] M.W. Tew, J.T. Miller, J.A. van Bokhoven, J. Phys. Chem. C 113 (2009) 15140-15147.

[425] J. Zhou, X. Zhou, X. Sun, R. Li, M. Murphy, Z. Ding, et al., Chem. Phys. Lett. 437 (2007) 229-232.

[426] F. Vila, J. Rehr, J. Kas, R. Nuzzo, A. Frenkel, Phys. Rev. B 78 (2008).

[427] Y. Lei, J. Jelic, L.C. Nitsche, R. Meyer, J. Miller, Top. Catal. 54 (2011) 334-348.

[428] K. Asakura, T. Kubota, W.J. Chun, Y. Iwasawa, K. Ohtani, T. Fujikawa, J. Synchrotron Rad. 6 (1999) 439-441.

[429] T. Kubota, K. Asakura, N. Ichikuni, Y. Iwasawa, Chem. Phys. Lett. 256 (1996) 445-448.

[430] T. Kubota, K. Asakura, Y. Iwasawa, Catal. Lett. 46 (1997) 141-144.

[431] N. Guo, B.R. Fingland, W.D. Williams, V.F. Kispersky, J. Jelic, W.N. Delgass, et al., Phys. Chem. Chem. Phys. 12 (2010) 5678-5693.

[432] S.N. Reifsnyder, M.M. Otten, D.E. Sayers, H.H. Lamb, J. Phys. Chem. B 101 (1997) 49724977.

[433] D.C. Koningsberger, J. de Graaf, B.L. Mojet, D.E. Ramaker, J.T. Miller, Appl. Catal. AGen. 191 (2000) 205-220.

[434] D.E. Starr, C.T. Campbell, J. Am. Chem. Soc. 130 (2008) 7321-7327.

[435] C.S. Kim, W.J. Tornquist, C. Korzeniewski, J. Chem. Phys. 101 (1994) 9113.

[436] B.E. Hayden, K. Kretzschmar, A.M. Bradshaw, R.G. Greenler, Surf. Sci. 149 (1985) 394406.

[437] B. Hvolbæk, T.V.W. Janssens, B.S. Clausen, H. Falsig, C.H. Christensen, J.K. Nørskov, Nano Today 2 (2007) 14-18.

[438] I.N. Remediakis, N. Lopez, J.K. N rskov, Angew. Chem. Int. Edit. 117 (2005) 18581860.

[439] G. Mpourmpakis, A.N. Andriotis, D.G. Vlachos, Nano Lett. 10 (2010) 1041-1045.

[440] J.A. Farmer, C.T. Campbell, Science 329 (2010) 933-936.

[441] J. Larsen, J. Ranney, D. Starr, J. Musgrove, C. Campbell, Phys. Rev. B 63 (2001) 195410. 118 
[442] J.A. Farmer, J.H. Baricuatro, C.T. Campbell, J. Phys. Chem. C 114 (2010) 17166-17172.

[443] Y. Xu, M. Mavrikakis, J. Phys. Chem. 107 (2003) 9298-9307.

[444] P. Muller, R. Kern, Appl. Surf. Sci. 164 (2000) 68-71.

[445] P. Muller, R. Kern, Surf. Sci. 457 (2000) 229-253.

[446] S. Giorgio, C. Chapon, C.R. Henry, G. Nihoul, Philos. Mag. B 67 (1993) 773-785.

[447] W. Vervisch, C. Mottet, J. Goniakowski, Eur. Phys. J. D 24 (2003) 311-314.

[448] X. Shao, S. Prada, L. Giordano, G. Pacchioni, N. Nilius, H.-J. Freund, Angew. Chem. Int. Edit. 50 (2011) 11525-11527.

[449] D. Ricci, A. Bongiorno, G. Pacchioni, U. Landman, Phys. Rev. Lett. 97 (2006).

[450] G. Pacchioni, L. Giordano, M. Baistrocchi, Physical Review Letters 94 (2005).

[451] B. Hammer, Top. Catal. 37 (2006) 3-16.

[452] K. Hayek, R. Kramer, Z. Paál, Applied Catalysis A: General 162 (1997) 1-15.

[453] D. Widmann, R.J. Behm, Accounts Chem. Res. 47 (2014) 740-749.

[454] L.B. Vilhelmsen, B. Hammer, ACS Catal. 4 (2014) 1626-1631.

[455] I.X. Green, W. Tang, M. Neurock, J.T. Yates, Science 333 (2011) 736-739.

[456] I.X. Green, W. Tang, M. Neurock, J.T. Yates, Angew. Chem. Int. Edit. 50 (2011) 1018610189.

[457] S. Hong, T.S. Rahman, J. Am. Chem. Soc. 135 (2013) 7629-7635.

[458] M. Farnesi Camellone, D. Marx, J. Phys. Chem. C 118 (2014) 20989-21000.

[459] P. Hirunsit, K.-i. Shimizu, R. Fukuda, S. Namuangruk, Y. Morikawa, M. Ehara, J. Phys. Chem. C 118 (2014) 7996-8006.

[460] X. Nie, H. Qian, Q. Ge, H. Xu, R. Jin, ACS Nano 6 (2012) 6014-6022.

[461] N.M. Schweitzer, J.A. Schaidle, O.K. Ezekoye, X. Pan, S. Linic, L.T. Thompson, J. Am. Chem. Soc. 133 (2011) 2378-2381.

[462] L. Cheng, C. Yin, F. Mehmood, B. Liu, J. Greeley, S. Lee, et al., ACS Catal. 4 (2014) 3239.

[463] G.N. Vayssilov, Y. Lykhach, A. Migani, T. Staudt, G.P. Petrova, N. Tsud, et al., Nat. Mater. 10 (2011) 310-315.

[464] M. Cargnello, V.V.T. Doan-Nguyen, T.R. Gordon, R.E. Diaz, E.A. Stach, R.J. Gorte, et al., Science 341 (2013) 771-773.

[465] H.Y. Kim, G. Henkelman, J. Phys. Chem. Lett. 3 (2012) 2194-2199.

[466] T. Yan, D.W. Redman, W.-Y. Yu, D.W. Flaherty, J.A. Rodriguez, C.B. Mullins, J. Catal. 294 (2012) 216-222.

[467] M.M. Schubert, S. Hackenberg, A.C. van Veen, M. Muhler, V. Plzak, R.J. Behm, J. Catal. 197 (2001) 113-122.

[468] H. Liu, A.I. Kozlov, A.P. Kozlova, T. Shido, Y. Iwasawa, Phys. Chem. Chem. Phys. 1 (1999) 2851-2860.

[469] A.F. Carley, D.J. Morgan, N. Song, M.W. Roberts, S.H. Taylor, J.K. Bartley, et al., Phys. Chem. Chem. Phys. 13 (2011) 2528.

[470] C. Becker, C.R. Henry, Surf. Sci. 352-354 (1996) 457-462. 
[471] S. Lin, M.A. Vannice, J. Catal. 143 (1993) 563-572.

[472] M. Bowker, D. James, P. Stone, R. Bennett, N. Perkins, L. Millard, et al., J. Catal. 217 (2003) 427-433.

[473] L.K. Ono, F. Behafarid, B.R. Cuenya, ACS Nano 7 (2013) 10327-10334.

[474] R.A. Bennett, C.L. Pang, N. Perkins, R.D. Smith, P. Morrall, R.I. Kvon, et al., J. Phys. Chem. B 106 (2002) 4688-4696.

[475] E. Wahlström, N. Lopez, R. Schaub, P. Thostrup, A. Rønnau, C. Africh, et al., Phys. Rev. Lett. 90 (2003).

[476] J.B. Park, S.F. Conner, D.A. Chen, J. Phys. Chem. C 112 (2008) 5490-5500.

[477] M. Bowker, R.A. Bennett, J. Phys. Condens. Mat. 21 (2009) 474224.

[478] R.A. Bennett, P. Stone, M. Bowker, Faraday Discuss. 114 (1999) 267-277.

[479] H. Watanabe, Jpn. J. Appl. Phys. 32 (1993) 2809-2813.

[480] L. Molina, B. Hammer, Phys. Rev. B 90 (2003).

[481] L. Molina, B. Hammer, Phys. Rev. B 69 (2004).

[482] A. Sanchez, S. Abbet, U. Heiz, W.D. Schneider, H. Häkkinen, R.N. Barnett, et al., J. Phys. Chem. A 103 (1999) 9573-9578.

[483] A. Del Vitto, G. Pacchioni, F.O. Delbecq, P. Sautet, J. Phys. Chem. B 109 (2005) 80408048.

[484] M. Sterrer, M. Yulikov, E. Fischbach, M. Heyde, H.-P. Rust, G. Pacchioni, et al., Angew. Chem. Int. Edit. 45 (2006) 2630-2632.

[485] C. Zhang, B. Yoon, U. Landman, J. Am. Chem. Soc. 129 (2007) 2228-2229.

[486] M. Cargnello, P. Fornasiero, R.J. Gorte, Catal. Lett. 142 (2012) 1043-1048.

[487] S.J. Tauster, S.C. Fung, R.L. Garten, J. Am. Chem. Soc. 100 (1978) 170-175.

[488] S.J. Tauster, S.C. Fung, J. Catal. 55 (1978) 29-35.

[489] H.R. Sadeghi, V.E. Henrich, Appl. Surf. Sci. 19 (1984) 330-340.

[490] H. Sadeghi, J. Catal. 109 (1988) 1-11.

[491] A. Berkó, I. Ulrych, K.C. Prince, J. Phys. Chem. B 102 (1998) 3379-3386.

[492] M. Bowker, E. Fourre, Appl. Surf. Sci. 254 (2008) 4225-4229.

[493] M. Bowker, P. Stone, P. Morrall, R. Smith, R. Bennett, N. Perkins, et al., J. Catal. 234 (2005) 172-181.

[494] M. Bowker, P. Stone, R. Bennett, N. Perkins, Surf. Sci. 497 (2002) 155-165.

[495] L. Giordano, G. Pacchioni, C. Noguera, J. Goniakowski, ChemCatChem 6 (2014) 185-190.

[496] A.K. Datye, D.S. Kalakkad, M.H. Yao, D.J. Smith, J. Catal. 155 (1995) 148-153.

[497] S. Bonanni, K. Aït-Mansour, H. Brune, W. Harbich, ACS Catal. 1 (2011) 385-389.

[498] F. Pesty, H.P. Steinruck, T.E. Madey, Surf. Sci. 339 (1995) 83-95.

[499] O. Dulub, W. Hebenstreit, U. Diebold, Phys. Rev. Lett. 84 (2000) 3646-3649.

[500] S. Arrii, F. Morfin, A.J. Renouprez, J.L. Rousset, J. Am. Chem. Soc. 126 (2004) 11991205.

[501] J.-D. Grunwaldt, A. Baiker, J. Phys. Chem. B 103 (1999) 1002-1012.

[502] S. Tsubota, T. Nakamura, K. Tanaka, M. Haruta, Catal. Lett. 56 (1998) 131-135. 
[503] J.-J. Zou, C. Chen, C.-J. Liu, Y.-P. Zhang, Y. Han, L. Cui, Mat. Lett. 59 (2005) 3437-3440.

[504] M.R. Hoffmann, S.T. Martin, W. Choi, D.W. Bahnemann, Chem. Rev. 95 (1995) 69-96.

[505] A. Furube, T. Asahi, H. Masuhara, H. Yamashita, M. Anpo, Chem. Phys. Lett. 336 (2001) 424-430.

[506] M. Lewandowski, Y.N. Sun, Z.H. Qin, S. Shaikhutdinov, H.J. Freund, Appl. Catal. A-Gen. 391 (2011) 407-410.

[507] M. Lewandowski, I.M.N. Groot, S. Shaikhutdinov, H.J. Freund, Catal. Today 181 (2012) 52-55.

[508] Y.N. Sun, Z.H. Qin, M. Lewandowski, E. Carrasco, M. Sterrer, S. Shaikhutdinov, et al., J. Catal. 266 (2009) 359-368.

[509] Y.-N. Sun, Z.-H. Qin, M. Lewandowski, S. Kaya, S. Shaikhutdinov, H.-J. Freund, Catal. Lett. 126 (2008) 31-35.

[510] Y.-N. Sun, L. Giordano, J. Goniakowski, M. Lewandowski, Z.-H. Qin, C. Noguera, et al., Angew. Chem. Int. Edit. 122 (2010) 4520-4523.

[511] Y. Martynova, S. Shaikhutdinov, H.-J. Freund, ChemCatChem 5 (2013) 2162-2166.

[512] A. Bruix, J.A. Rodriguez, P.J. Ramírez, S.D. Senanayake, J. Evans, J.B. Park, et al., J. Am. Chem. Soc. 134 (2012) 8968-8974.

[513] M. Ritter, W. Ranke, W. Weiss, Phys. Rev. B 57 (1998) 7240-7251.

[514] H.C. Galloway, P. Sautet, M. Salmeron, Phys. Rev. B 54 (1996) 11145-11148.

[515] R. Imbihl, G. Ertl, Chem. Rev. 95 (1995) 697-733.

[516] X.-Q. Gong, Z.-P. Liu, R. Raval, P. Hu, J. Am. Chem. Soc. 126 (2004) 8-9.

[517] V. Tagliazucca, K. Schlichte, F. Schüth, C. Weidenthaler, J. Catal. 305 (2013) 277-289.

[518] M. Feyen, C. Weidenthaler, R. Güttel, K. Schlichte, U. Holle, A.-H. Lu, et al., Chem. Eur. J. 17 (2011) 598-605.

[519] L. Geng, X. Zhang, W. Zhang, M. Jia, G. Liu, Chem. Commun. 50 (2014) 2965.

[520] Z. Ma, F. Zaera, Heterogeneous Catalysis by Metals, Encyclopedia of Inorganic and Bioinorganic Chemistry, John Wiley \& Sons, Ltd., 2011.

[521] C.A. Carrero, R. Schloegl, I.E. Wachs, R. Schomaecker, ACS Catal. 4 (2014) 3357-3380.

[522] M. Høj, T. Kessler, P. Beato, A.D. Jensen, J.-D. Grunwaldt, Appl. Catal. A-Gen. 472 (2014) 29-38.

[523] Y. Meng, T. Wang, S. Chen, Y. Zhao, X. Ma, J. Gong, Appl. Catal. B-Environ. 160-161 (2014) 161-172.

[524] P. Mars, D.W. Van Krevelen, Chem. Eng. Sci. 3 (1954) 41-59.

[525] C.T. Campbell, Phys. Rev. Lett. 96 (2006).

[526] H. Kuhlenbeck, G. Odörfer, R. Jaeger, G. Illing, M. Menges, T. Mull, et al., Phys. Rev. B 43 (1991) 1969-1986.

[527] S.P. Devarajan, J.A. Hinojosa, J.F. Weaver, Surf. Sci. 602 (2008) 3116-3124.

[528] E. Lundgren, G. Kresse, C. Klein, M. Borg, J. Andersen, M. De Santis, et al., Phys. Rev. Lett. 88 (2002) 246103.

[529] Y. Xu, W.A. Shelton, W.F. Schneider, J. phys. Chem. A 110 (2006) 5839-5846.

[530] Y. Xu, W.A. Shelton, W.F. Schneider, J. Phys. Chem. B 110 (2006) 16591-16599. 
[531] L.K. Ono, B. RoldanCuenya, J. Phys. Chem. C 112 (2008) 4676-4686.

[532] X.-Q. Gong, R. Raval, P. Hu, Phys. Rev. Lett. 93 (2004).

[533] J.Y. Park, K. Qadir, S.M. Kim, in, Current Trends of Surface Science and Catalysis, Springer New York, 2014, pp. 145-170.

[534] Y. Martynova, B. Yang, X. Yu, J.A. Boscoboinik, S. Shaikhutdinov, H.J. Freund, Catal. Lett. 142 (2012) 657-663.

[535] H.I.W. Lee, J.M., J. Catal. 63 (1980) 261-264.

[536] S. Wendt, M. Knapp, H. Over, J. Am. Chem. Soc. 126 (2004) 1537-1541.

[537] C.H.F. Peden, D.W. Goodman, J. Phys. Chem. 90 (1986) 1360-1365.

[538] C. Stampfl, S. Schwegmann, H. Over, M. Scheffler, G. Ertl, Phys. Rev. Lett. 77 (1996) 3371-3374.

[539] C. Stampfl, M. Scheffler, Phys. Rev. B 54 (1996) 2868-2872.

[540] A. Böttcher, H. Niehus, S. Schwegmann, H. Over, G. Ertl, J. Phys. Chem. B 101 (1997) 11185-11191.

[541] H. Over, Y.D. Kim, A.P. Seitsonen, S. Wendt, E. Lundgren, M. Schmid, et al., Science 287 (2000) 1474-1476.

[542] K. Reuter, D. Frenkel, M. Scheffler, Phys. Rev. Lett. 93 (2004).

[543] S.I. Shah, S. Hong, T.S. Rahman, J. Phys. Chem. C 118 (2014) 5226-5238.

[544] J. Aßmann, V. Narkhede, N.A. Breuer, M. Muhler, A.P. Seitsonen, M. Knapp, et al., J. Phys. Condens. Mat. 20 (2008) 184017.

[545] J. Aßmann, V. Narkhede, L. Khodeir, E. Löffler, O. Hinrichsen, A. Birkner, et al., J. Phys. Chem. B 108 (2004) 14634-14642.

[546] J. Aßmann, D. Crihan, M. Knapp, E. Lundgren, E. Loffler, M. Muhler, et al., Angew. Chem. Int. Edit. 44 (2005) 917-920.

[547] J. Aßmann , D. Crihan, M. Knapp, E. Lundgren, E. Löffler , M. Muhler, et al., Angew. Chem. 117 (2005) 939-942.

[548] J. Aßmann, E. Löffler, A. Birkner, M. Muhler, Catal. Today 85 (2003) 235-249.

[549] V. Narkhede, J. Aßmann, M. Muhler, Z. Phys. Chem. 219 (2005) 979-995.

[550] M.A. Newton, K.W. Chapman, D. Thompsett, P.J. Chupas, J. Am. Chem. Soc. 134 (2012) 5036-5039.

[551] B. Hendriksen, J. Frenken, Phys. Rev. Lett. 89 (2002) 046101.

[552] E.M. Cordi, J.L. Falconer, J. Catal. 162 (1996) 104-117.

[553] Z. Duan, G. Henkelman, ACS Catal. (2014) 3435-3443.

[554] J. Stötzel, R. Frahm, B. Kimmerle, M. Nachtegaal, J.-D. Grunwaldt, J. Phys. Chem. C 116 (2012) 599-609.

[555] R. Imbihl, Prog. Surf. Sci. 44 (1993) 185-343.

[556] F. Schüth, B.E. Henry, L.D. Schmidt, Adv. Catal. 39 (1993) 51-127.

[557] M.M. Slin'ko, N.I. Jaeger, Oscillating heterogeneous catalytic systems, Elsevier, Amsterdam ; New York, 1994.

[558] P. Ciambelli, A. Di Benedetto, R. Pirone, G. Russo, Chem. Eng. Sci. 54 (1999) 2555-2559.

[559] P. Ciambelli, J. Catal. 175 (1998) 161-169.

122 
[560] E.M. Slavinskaya, O.A. Stonkus, R.V. Gulyaev, A.S. Ivanova, V.I. Zaikovskii, P.A. Kuznetsov, et al., Appl. Catal. A-Gen. 401 (2011) 83-97.

[561] E.A. Lashina, E.M. Slavinskaya, N.A. Chumakova, O.A. Stonkus, R.V. Gulyaev, A.I. Stadnichenko, et al., Chem. Eng. Sci. 83 (2012) 149-158.

[562] S.B. Vendelbo, C.F. Elkjær, H. Falsig, I. Puspitasari, P. Dona, L. Mele, et al., Nat. Mater. 13 (2014) 884-890.

[563] E. Wicke, P. Kummann, W. Keil, J. Schiefler, Ber. Bunsenges. Phys. Chem. 84 (1980) 315-323.

[564] J.E. Turner, M.B. Maple, Surf. Sci. 147 (1984) 647-662.

[565] B.L.M. Hendriksen, M.D. Ackermann, R. van Rijn, D. Stoltz, I. Popa, O. Balmes, et al., Nat. Chem. 2 (2010) 730-734.

[566] R. Imbihl, M.P. Cox, G. Ertl, J. Chem. Phys. 84 (1986) 3519.

[567] M. Eiswirth, G. Ertl, Surf. Sci. 177 (1986) 90-100.

[568] B. Hendriksen, S. Bobaru, J. Frenken, Catal. Today 105 (2005) 234-243.

[569] J.E. Turner, B.C. Sales, M.B. Maple, Surf. Sci. 109 (1981) 591-604.

[570] E. Fridell, A. Amberntsson, L. Olsson, A.W. Grant, M. Skoglundh, Top. Catal. 30/31 (2004) 143-146.

[571] S. Mulla, N. Chen, L. Cumaranatunge, G. Blau, D. Zemlyanov, W. Delgass, et al., J. Catal. 241 (2006) 389-399.

[572] L. Olsson, J. Catal. 210 (2002) 340-353.

[573] S. Salomons, R.E. Hayes, M. Votsmeier, A. Drochner, H. Vogel, S. Malmberg, et al., Appl. Catal. B-Environ. 70 (2007) 305-313.

[574] W. Hauptmann, M. Votsmeier, J. Gieshoff, A. Drochner, H. Vogel, Appl. Catal. BEnviron. 93 (2009) 22-29.

[575] J. Després, M. Elsener, M. Koebel, O. Kröcher, B. Schnyder, A. Wokaun, Appl. Catal. BEnviron. 50 (2004) 73-82.

[576] Y. Xu, R.B. Getman, W.A. Shelton, W.F. Schneider, Phys. Chem. Chem. Phys. 10 (2008) 6009.

[577] S. Bals, M. Casavola, M.A. van Huis, S. Van Aert, K.J. Batenburg, G. Van Tendeloo, et al., Nano Lett. 11 (2011) 3420-3424.

[578] S. Van Aert, K.J. Batenburg, M.D. Rossell, R. Erni, G. Van Tendeloo, Nature 470 (2011) 374-377.

[579] H. Yang, J.B. Wu, A. Gross, Nano Lett. 11 (2011) 798-802.

[580] Q. Yuan, Z. Zhou, J. Zhuang, X. Wang, Inorg. Chem. 49 (2010) 5515-5521.

[581] A.M. Karim, V. Prasad, G. Mpourmpakis, W.W. Lonergan, A.I. Frenkel, J.G. Chen, et al., J. Am. Chem. Soc. 131 (2009) 12230-12239.

[582] R. Wang, H. He, L.-C. Liu, H.-X. Dai, Z. Zhao, Catal. Sci. Technol. 2 (2012) 575-580.

[583] X. Gong, Y. Yang, L. Zhang, C. Zou, P. Cai, G. Chen, et al., J. Colloid Interf. Sci. 352 (2010) 379-385.

[584] R.V. Chepulskii, S. Curtarolo, ACS Nano 5 (2011) 247-254.

[585] P. Christopher, S. Linic, J. Am. Chem. Soc. 130 (2008) 11264-11265. 
[586] C. Susut, Y.J. Tong, Electrocatal. 2 (2011) 75-81.

[587] Z.-N. Xu, J. Sun, C.-S. Lin, X.-M. Jiang, Q.-S. Chen, S.-Y. Peng, et al., ACS Catal. 3 (2013) 118-122.

[588] C. Wang, H. Daimon, Y. Lee, J. Kim, S. Sun, J. Am. Chem. Soc. 129 (2007) 6974-6975.

[589] J. Hernandez, J. Solla-Gullon, E. Herrero, A. Aldaz, J.M. Feliu, J. Phys. Chem. C 111 (2007) 14078-14083.

[590] S. Linic, P. Christopher, ChemCatChem 2 (2010) 1061-1063.

[591] S. Linic, P. Christopher, H. Xin, A. Marimuthu, Accounts Chem. Res. 46 (2013) 18901899.

[592] J.-Y. Ho, M.H. Huang, J. Phys. Chem. C 113 (2009) 14159-14164.

[593] V.P. Zhdanov, B. Kasemo, Surf. Sci. Rep. 39 (2000) 25-104.

[594] V. Komanicky, A. Menzel, K.-C. Chang, H. You, J. Phys. Chem. B 109 (2005) 2354323549.

[595] C.J. Kliewer, M. Bieri, G.A. Somorjai, J. Phys. Chem. C 112 (2008) 11373-11378.

[596] H. Zhou, I. Honma, H. Komiyama, J. Haus, Phys. Rev. B 50 (1994) 12052-12056.

[597] J.J. Mock, M. Barbic, D.R. Smith, D.A. Schultz, S. Schultz, J. Chem. Phys. 116 (2002) 6755-6759.

[598] R. Jin, Y. Cao, C.A. Mirkin, K.L. Kelly, G.C. Schatz, J.G. Zheng, Science 294 (2001) 1901-1903.

[599] R. Jin, Y. Charles Cao, E. Hao, G.S. Métraux, G.C. Schatz, C.A. Mirkin, Nature 425 (2003) 487-490.

[600] B.J. Wiley, S.H. Im, Z.-Y. Li, J. McLellan, A. Siekkinen, Y. Xia, J. Phys. Chem. B 110 (2006) 15666-15675.

[601] C.M. Cobley, S.E. Skrabalak, D.J. Campbell, Y.N. Xia, Plasmonics 4 (2009) 171-179.

[602] Y. Xia, N.J. Halas, MRS Bulletin 30 (2011) 338-348.

[603] C. Noguez, J. Phys. Chem. C 111 (2007) 3806-3819.

[604] S. Eustis, M.A. El-Sayed, Chem. Soc. Rev. 35 (2006) 209.

[605] M.-C. Daniel, D. Astruc, Chem. Rev. 104 (2004) 293-346.

[606] M.A. Mackey, M.R.K. Ali, L.A. Austin, R.D. Near, M.A. El-Sayed, J Phys. Chem. B 118 (2014) 1319-1326.

[607] L.R. Hirsch, R.J. Stafford, J.A. Bankson, S.R. Sershen, B. Rivera, R.E. Price, et al., Proc. Natl. Acad. Sci. USA 100 (2003) 13549-13554.

[608] Y.-L. Luo, Y.-S. Shiao, Y.-F. Huang, ACS Nano 5 (2011) 7796-7804.

[609] M.E. Stewart, C.R. Anderton, L.B. Thompson, J. Maria, S.K. Gray, J.A. Rogers, et al., Chem. Rev. 108 (2008) 494-521.

[610] J.N. Anker, W.P. Hall, O. Lyandres, N.C. Shah, J. Zhao, R.P. Van Duyne, Nat. Mater. 7 (2008) 442-453.

[611] Z. Liu, W. Hou, P. Pavaskar, M. Aykol, S.B. Cronin, Nano Lett. 11 (2011) 1111-1116.

[612] J.R. Adleman, D.A. Boyd, D.G. Goodwin, D. Psaltis, Nano Lett. 9 (2009) 4417-4423.

[613] P. Xu, L. Kang, N.H. Mack, K.S. Schanze, X. Han, H.-L. Wang, Sci. Rep. 3 (2013) 2997. 
[614] C. Hu, T. Peng, X. Hu, Y. Nie, X. Zhou, J. Qu, et al., J. Am. Chem. Soc. 132 (2010) 857862.

[615] Y. Tsuboi, R. Shimizu, T. Shoji, N. Kitamura, J. Am. Chem. Soc. 131 (2009) 1262312627.

[616] S. Linic, P. Christopher, D.B. Ingram, Nat. Mater. 10 (2011) 911-921.

[617] K.L. Kelly, E. Coronado, L.L. Zhao, G.C. Schatz, J. Phys. Chem. B 107 (2003) 668-677.

[618] P.K. Jain, X.H. Huang, I.H. El-Sayed, M.A. El-Sayed, Accounts Chem. Res. 41 (2008) 1578-1586.

[619] K. Watanabe, D. Menzel, N. Nilius, H.-J. Freund, Chem. Rev. 106 (2006) 4301-4320.

[620] U. Kreibig, M. Vollmer, Optical Properties of Metal Clusters, Springer Berlin Heidelberg, 1995.

[621] A. Marimuthu, J. Zhang, S. Linic, Science 339 (2013) 1590-1593.

[622] P. Christopher, H. Xin, S. Linic, Nat. Chem. 3 (2011) 467-72.

[623] P. Christopher, H.L. Xin, A. Marimuthu, S. Linic, Nat. Mater. 11 (2012) 1044-1050.

[624] M.D. Porosoff, X. Yang, J.A. Boscoboinik, J.G. Chen, Angew. Chem. Int. Edit. 126 (2014) 6823-6827. 